\title{
Dynamics of Scaffold Protein Tethered Signal Transduction
}

\begin{tabular}{c} 
A Dissertation \\
Presented to \\
the faculty of the School of Engineering and Applied Science \\
University of Virginia \\
\hline
\end{tabular}

in partial fulfillment

of the requirements for the degree

Doctor of Philosophy

by

Eric Chris Greenwald

May 2015 


\section{APPROVAL SHEET}

This dissertation is in partial fulfillment of the requirements for the degree of Doctor of Philosophy in Biomedical Engineering

Eric Chris Greenwald

Author

This dissertation has been read and approved by the examining committee:

Jeffrey J. Saucerman, Ph.D.

Dissertation Advisor

Department of Biomedical Engineering

Brian P. Helmke, Ph.D.

Committee Chair

Department of Biomedical Engineering

Kevin A. Janes, Ph.D.

Committee Member

Department of Biomedical Engineering

Jason A. Papin, Ph.D.

Committee Member

Department of Biomedical Engineering

David L. Brautigan, Ph.D.

Committee Member, Department of Microbiology, Immunology, and Cancer Biology

Accepted for the School of Engineering and Applied Science:

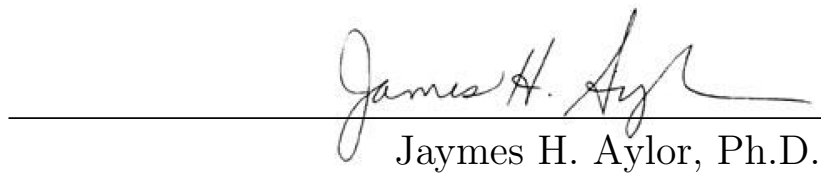

Dean, School of Engineering and Applied Science

May 2015 
This thesis is dedicated to

Hannah, Mom, Dad, A.J. and Gretchen,

whose support provided me the strength and drive to reach my goals.

Prediction, not narration, is the real test of our understanding of the world.

-Nassim Nicholas Taleb, The Black Swan

I was bold in the pursuit of knowledge, never fearing to follow truth and reason to whatever results they led.

-Thomas Jefferson 


\section{Abstract}

The basis of intracellular communication and information processing lies in highly connected and complex networks of signal transduction that can produce diverse responses to stimuli. Scaffold proteins bind two or more signaling proteins and play a key role in spatially and temporally organizing these networks of signal transduction. By tethering multiple signaling proteins in close proximity, scaffolds have been hypothesized to be an important determinant of signaling specificity and efficiency. While scaffolds have been observed to amplify and accelerate signaling dynamics, an open challenge remains to identify a mechanistic explanation for these scaffold-derived emergent phenomena. Additionally, the coordination of several interacting proteins can obscure the functional role of a given scaffold protein. Through the combination of computational modeling and live-cell imaging, this dissertation aims to investigate the effects of scaffold proteins on signaling dynamics, and the mechanism that underlies them.

To address the amplification and acceleration of scaffold tethered signaling, we propose the novel "scaffold state-switching" mechanism, where the enzyme-substrate-scaffold complex can stochastically switch between active and inactive intermediate states before the enzyme completes catalysis. We developed a computational model of this mechanism showing that scaffold proteins can amplify and accelerate tethered signal transduction by increasing the rate of enzyme-substrate interaction. To validate these predictions we exploited 
a direct interaction between Protein Kinase $\mathrm{C}(\mathrm{PKC})$ and $\mathrm{AKAP} 7 \alpha$ and found that both the strength and speed of substrate phosphorylation were enhanced in agreement with the computational model. Additionally, extension of this model to study the effects of scaffold proteins on inhibitors led to the prediction and subsequent validation that scaffold proteins can insulate tethered enzymes from substrate- and ATP-competitive inhibitors but not activation-competitive inhibitors. Together, these data provide theoretical and experimental evidence that scaffold proteins can amplify, accelerate and insulate signal transduction through the scaffold state-switching mechanism.

To investigate the coordination of multiple signaling pathways by scaffold proteins, we studied the role of AKAP5 in the coordination of crosstalk between oscillatory Protein Kinase A (PKA) and calcium signaling in MIN6 $\beta$-cells. Using FRET biosensors, we show that Protein Kinase A activity at the plasma membrane oscillates out-of-phase with calcium whereas AKAP5-anchored PKA oscillates in-phase with calcium. The mechanism of these unique dynamics were studied through the development of computational models testing different hypotheses for the role of AKAP5. These models predicted that by regulating the activation of two distinct pools of adenylyl cyclase, AKAP5 simultaneously coordinates both the in- and out-of-phase PKA activity. Extension of this model also identified that the AKAP5 coordination of the positive feedback of PKA onto the Cav 1.2 calcium channel is necessary for the development of calcium oscillations. Through the application of these computational models, these data show that the coordination of a specific network of signaling proteins by AKAP5 enable the development of unique signaling dynamics and make AKAP5 essential for the oscillatory dynamics in these cells.

Together, this body of work provides insight into both the mechanisms of scaffold tethered signal transduction and the effects that can arise from this tethering. In addition to improving our understanding of the very basis of cell signaling, this work provides a quanti- 
tative framework with which to analyze the effects of all scaffold proteins. This framework will be instrumental in evaluating the disruption of scaffold interactions as a therapeutic strategy and identifying which interactions to target. 


\section{Acknowledgments}

I would first like to extend my deepest gratitude to my advisor, Jeff Saucerman. You have worked diligently to help me utilize my strengths and pushed me to develop my weaknesses, and I am much better for it. I have enjoyed our many discussions on modeling, MATLAB and figure design and I aspire to be as great of a mentor, teacher and scientist as you have shown me you are.

Throughout my time here at The University of Virginia, I have received support and encouragement from colleagues in the BME department and across the university. First, I would like to thank my Thesis Committee (Brian Helmke, David Brautigan, Jason Papin and Kevin Janes ) for all of your feedback and advice, both scientific and personal. Additionally, the discussions with the faculty in the Systems Biology Journal Club have been informational and really shaped my view of the direction of the field. Thank you to all of my fellow graduate students, you have helped make graduate school a very enjoyable and rewarding journey. Furthermore, I am lucky to have made so many good friends here and would like

to especially thank Sameer Bajikar, Scott Seaman and Edik Blais, for keeping me sane and always being willing to grab a beer, even if I may over-analyze it. I am also appreciative of the many discussions, lessons, and experiences I have had with the TPT program, the School of Engineering teaching fellowship and with Lindsey Brinton that have really shaped my view of teaching, the role of the faculty and career decisions. 
To my fellow Saucerman lab-mates, it has been a pleasure to work along side all of you, through both the shared frustrations and joys of research. Jason Yang, thank you for being both a friend and a mentor. You inspire me to reach out and connect with new people and to never be afraid to tackle the big guys. To Robert Amanfu, Karen Ryall, and Renata Polanowska-Grabowska, thank you for showing me the ropes, providing support and feedback, and making the lab fun to be in. And finally, to the current Saucerman graduate students Angela Zeigler, Philip Tan, Laura Woo and Bryan Chun, I want to impress that you should keep pushing forward even in the face of experiments that aren't working because even if it doesn't turn out exactly as you planned, you will make it in the end. Also, don't be afraid to make sure all the first floor can hear you laughing.

There are also several people outside of the university that have been fundamental to me reaching this goal. I am indebted to the many collaborators for their work on these projects: Kim Dodge-Kafka, John Redden, Jin Zhang, Brian Tenner and Sohum Mehta. Also, I would like to thank Stephanie Bryant and Garret Nicodemus giving me the opportunity to get started in research, for providing me career advice, and their continued support. My original interest in research was probably seeded by two of my most influential teachers, Adam Norris and Rob Matuschek, and my accomplishments here are at least partial reflections of their effects on me when I was younger.

Upon looking back at this thesis it really makes me appreciate all the support I have had throughout my entire life. Most recently, I have been really glad to be a part of the Virginia Rugby Football Club family and thank them for pushing me to do things that are both smart and possibly questionable, especially Jason Gaviria, "Pappy", Brian Carver and coach Tom. Growing up I have been part of an extended family that formed a great support network so thank you to the Kirby, Rowland, Roberts and Hanes families. To my brother A.J., whether it was experiments testing how much gum could fit in a mouth (subjects $n=2$ ) 
or trying to determine the speed of light between the door and the bed, you have always been at my side supporting the scientist that I am. To my sister Gretchen, you always made sure that I knew you loved me and I knew I could always count on you for a hug. Thank you to the both of you. Mom and Dad, you have always pushed me to try my hardest at everything I do and the hard work that I put into this thesis is a direct reflection of the work you put into raising me. To all my friends and family, thank you and I love you all.

And finally, to my wife Hannah: Thank you for your love, support, patience and motivation. We may have not have taken the easiest road but we are stronger for it. This thesis marks the end of a chapter but beginning of a new life together. I can't wait and I loves you.

Eric C. Greenwald May 2015

This work has been made possible by the generosity of the University of Virginia, NIH Pharmacological Sciences Training Grant and the UVA School of Medicine Wagner Fellowship. 


\section{Contents}

Abstract

Acknowledgments

Contents $\quad$ ix

List of Figures

List of Tables $\quad$ xvi

Abbreviations $\quad$ xvii

1 Dissertation Aims $\quad 1$

1.1 Introduction . . . . . . . . . . . . . . . . . 2

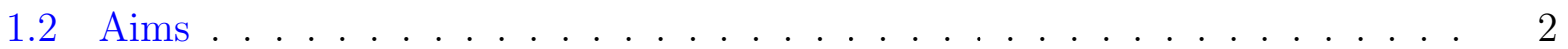

2 Background and Significance $\quad 4$

2.1 Foreword . . . . . . . . . . . . . . . . . . . . 5

2.2 Introduction . . . . . . . . . . . . . . . . . . 5

2.3 Design Principles for AKAP Function . . . . . . . . . . . . . . 6

2.4 Network Motifs on AKAP Signaling Complexes . . . . . . . . . . . . . . . 11

2.5 Computational Models of AKAP Signaling . . . . . . . . . . . . . . . 16

2.6 Computational Models of Other Signaling Scaffolds . . . . . . . . . . . . 19

2.7 Conclusions . . . . . . . . . . . . . . . . . . . . . . . 23

3 Integ. Fluo. Biosensor Data Using Comp. Models 25

3.1 Foreword . . . . . . . . . . . . . . . . . 26 
3.2 Introduction . . . . . . . . . . . . . . . . . 26

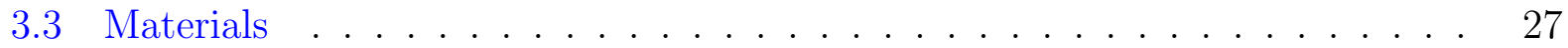

3.4 Model Generation . . . . . . . . . . . . . . . . . . . . . . . . . . . . . 29

3.5 Collection of FRET Data for Model Integration . . . . . . . . . . . 40

3.6 Integrating Experimental Data . . . . . . . . . . . . . . . 47

3.7 Interpreting Biosensor Data Using a Model . . . . . . . . . . . . . . 52

3.8 Conclusions . . . . . . . . . . . . . . . . . . . . . . . 54

4 Scaffold state-switching amplifies, accelerates and insulates PKC signaling 56

4.1 Foreword . . . . . . . . . . . . . . . . . . 57

4.2 Introduction . . . . . . . . . . . . . . . . . 57

4.3 Methods . . . . . . . . . . . . . . . . . . . . . . 59

4.4 Results . . . . . . . . . . . . . . . . . . . . . 61

4.4.1 Scaffold State-Switching Model Predicts Amplification and Acceleration of Reactions Occurring on Scaffolds . . . . . . . . . . . . 61

4.4.2 AKAP7 $\alpha$ amplifies substrate phosphorylation by PKC as predicted by the Scaffold State-Switching model . . . . . . . . . . . . . . . 64

4.4.3 AKAP7 $\alpha$ insulates PKC from substrate- and ATP-competitive inhibitors but not activation-competitive inhibitors . . . . . . 68

4.4.4 Extent of Acceleration, Amplification and Insulation Vary Depending on Enzyme Kinetics . . . . . . . . . . . . . . . . . . 73

4.5 Discussion . . . . . . . . . . . . . . . . . . . . . . . 74

5 AKAP5 coordinates both in- and out-of-phase PKA and $\mathrm{Ca}^{2+}$ oscillations 79

5.1 Foreword . . . . . . . . . . . . . . . . 80

5.2 Introduction . . . . . . . . . . . . . . . . . 80

5.3 Methods . . . . . . . . . . . . . . . . . . . . . 82

5.4 Results . . . . . . . . . . . . . . . . . . . . 86

5.4.1 Disruption of AKAP5 or PKA tethering decreases regular TEA induced calcium oscillations . . . . . . . . . . . . 86

5.4.2 AKAP5-tethered PKA oscillates in-phase with $\mathrm{Ca}^{2+} \ldots \ldots \ldots . .87$

5.4.3 Model requires $\mathrm{Ca}^{2+}$ induced activation of PKA to agree with experimental data . . . . . . . . . . . . . . . . . . 90 
5.4.4 Diffusion of cAMP between compartments is insufficient for robust plasma membrane PKA oscillations . . . . . . . . . . . . . . . . . . 92

5.4.5 Differences in phosphatase concentration inconsistent with sharp decrease in PKA activity . . . . . . . . . . . . . . . . . . . 93

5.4.6 AKAP5-coordinated activation of AC8 and AC5 concords with all five observed oscillatory features . . . . . . . . . . . . . . 95

5.4.7 Computational model predicts that AKAP5-tethered PKA is necessary for TEA induced calcium oscillations . . . . . . . . . . . . . . . . . . 97

5.5 Discussion . . . . . . . . . . . . . . . . . . 101

6 Dissertation discussion $\quad 106$

6.1 Global Aims . . . . . . . . . . . . . . . . . . . 107

6.1.1 Dynamics of AKAP7 $\alpha$ and AKAP5 tethered signal transduction . . . 107

6.1.2 Relevance to other scaffold proteins . . . . . . . . . . . . . . 109

6.2 Future directions . . . . . . . . . . . . . . . . . . . . . . . . . 114

6.2.1 Additional signaling proteins and scaffolds . . . . . . . . . . . . . 114

6.2.2 Scaffold-tethered signaling motifs . . . . . . . . . . . . . . . 115

6.2 .3 (Re)structure of signaling networks . . . . . . . . . . . . . 117

6.2.4 Drug targets and synthetic scaffolds . . . . . . . . . . . . . 118

6.3 Conclusions . . . . . . . . . . . . . . . . . . . . . 119

A Scaffold state-switching model description $\quad 121$

A.1 Model Description . . . . . . . . . . . . . . . . . . . . . 122

A.1.1 Free Model . . . . . . . . . . . . . . . . . . . . 123

A.1.2 Scaffold State-Switching Model . . . . . . . . . . . . . . . . . 124

A.1.3 Model Fitting . . . . . . . . . . . . . . . . . . 127

A.2 Model extension to include enzyme inhibitors . . . . . . . . . . . . . . 129

A.2.1 Free Model . . . . . . . . . . . . . . . . . . . 129

A.2.2 Scaffold Model . . . . . . . . . . . . . . . . . 132

A.2.3 Model Interrogation of Insulation Mechanisms . . . . . . . . . . . . . 134

A.2.4 Model Sensitivity Analysis . . . . . . . . . . . . . . . . 136

B AKAP5 model description $\quad 142$

B.1 EP module . . . . . . . . . . . . . . . . . . . . 142 
B.1.1 TEA Stimulated $\mathrm{Ca}^{2+}$ Oscillation . . . . . . . . . . . . . . 142

B.1.2 Feedback by PKA . . . . . . . . . . . . . . . . . . . 143

B.2 CaM Module . . . . . . . . . . . . . . . . . . . . . . . . . 144

B.3 PKA Module definition . . . . . . . . . . . . . . . . . . . . . 144

B.3.1 PKA equations . . . . . . . . . . . . . . . . 145

B.3.2 cAMP degradation . . . . . . . . . . . . . . . 146

B.3.3 cAMP Production . . . . . . . . . . . . . . . . . 148

B.3.4 Other Signaling . . . . . . . . . . . . . . . . . . 150

B.3.5 cAMP ODEs . . . . . . . . . . . . . . . . 151

$\begin{array}{lr}\text { Bibliography } & 157\end{array}$ 


\section{List of Figures}

\section{Background and Significance}

2.1 Design principles of AKAP signaling . . . . . . . . . . . 7

2.2 Examples of complex signaling responses that are possible on AKAPs. . . . 15

3 Integ. Fluo. Biosensor Data Using Comp. Models

3.1 Virtual Cell Structure and Reaction Diagram _. . . . . . . . . . . . 34

3.2 Initial Simulation Results . . . . . . . . . . . . . . . . . . . . 41

3.3 Experimental Data . . . . . . . . . . . . . . . . . . . . 45

3.4 Data Normalization and Fitting . . . . . . . . . . . . . . . . 49

3.5 Testing hypotheses in silico . . . . . . . . . . . . . . . . 55

4 Scaffold state-switching amplifies, accelerates and insulates PKC signaling

4.1 The scaffold state-switching model . . . . . . . . . . . . . . . . 63

4.2 State switching model predicts acceleration and amplification of substrate phosphorylation . . . . . . . . . . . . . . . 65

4.3 AKAP $7 \alpha$ accelerates and amplifies CKAR phosphorylation. . . . . . . . 67

4.4 Co-expression of AKAP7 $\alpha$ does not affect responses of MyrCKAR. . . . . 67

4.5 AKAP7 $\alpha$-CKAR does not have a larger dynamic range than MyrCKAR. . . 69

4.6 State-switching model predicts insulation of anchored PKC. . . . . . . . . 69

4.7 PKC tethered to AKAP7 $\alpha$ is insulated from certain classes of pharmacological

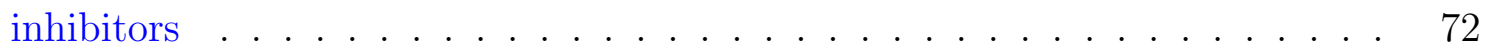

4.8 Amplification, acceleration and insulation vary depending on enzyme kinetics 75 


\section{AKAP5 coordinates both in- and out-of-phase PKA and $\mathrm{Ca}^{2+}$ oscillations}

5.1 Disruption of scaffold tethering reduces regular $\mathrm{Ca}^{2+}$ oscillations . . . . . 88

5.2 AKAP5-tethered PKA activity oscillates in-phase with $\mathrm{Ca}^{2+} \ldots$. . . . . 89

5.3 Quantification of PKA to $\mathrm{Ca}^{2+}$ temporal lag. . . . . . . . . . . . . 89

5.4 Previous model exhibits out-of-phase PM PKA oscillations that decrease from

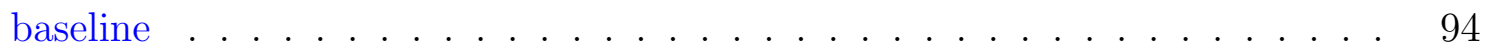

5.5 cAMP Diffusion model exhibits correct phase but small PKA dynamics . . . 94

5.6 Phosphatase model oscillations are insensitive to decreases in PKA activity. . 96

5.7 AC8/AC5 model agrees with all qualitative metrics seen experimentally. . . . 98

5.8 Positive feedback by AKAP5-tethered PKA necessary for TEA-induced $\mathrm{Ca}^{2+}$ oscillations . . . . . . . . . . . . . . . . . . . 100

\section{Dissertation discussion}

6.1 Scaffold proteins coordinate most of the key signaling proteins in the cardiac $\beta$-adrenergic signaling pathway. . . . . . . . . . . . . . . . . 120

\section{A Scaffold state-switching model description}

A.1 Model reaction diagram. . . . . . . . . . . . . . . . . . . . . . . . . . 122

A.2 Fitting of the model to the data. . . . . . . . . . . . . . . . 129

A.3 Network diagram of the extended model with activation-competitive, substratecompetitive, and ATP-competitive inhibitors. . . . . . . . . . . . . . 130

A.4 Dose-dependent effects of the substrate-competitive inhibitor on the active intermediate concentration. . . . . . . . . . . . . . . . 135

A.5 The scaffold creates a "catalytic reserve," where CKAR phosphorylation is less sensitive to decreases in $k_{\text {cat }}$. . . . . . . . . . . . . . . . . 135

A.6 Example predictions from hypothetical scaffold signaling complexes. . . . . . 138

A.7 Individual model parameters do not directly correlate to scaffold response. . 139

\section{B AKAP5 model description}

B.1 Details of PKA signaling in the model . . . . . . . . . . . . . . . 153

B.2 Details of the Ni 2011 signaling model . . . . . . . . . . . . . . . . . 153

B.3 Details of the cAMP Diffusion signaling model . . . . . . . . . . . . 154 
B.4 Details of the Phosphatase signaling model . . . . . . . . . . . . . . 155

B.5 Details of the AC8/AC5 signaling model . . . . . . . . . . . 156 


\section{List of Tables}

2.1 Key Systems Concepts Relevant to Understanding AKAP Signaling . . . . . 16

3.1 Initial parameter values defined for the example model. . . . . . . . . . . . 36

3.2 Initial concentrations . . . . . . . . . . . . . . . . . . 38

$5.1 \quad$ Features of Experimental PKA Oscillations. . . . . . . . . . . . . . . . . 99

A.1 Definition of model species . . . . . . . . . . . . . . . . . . . . . 122

A.2 Constant model parameters. . . . . . . . . . . . . . . . . . . 127

A.3 Fitted model parameters. . . . . . . . . . . . . . . . . . . . . 127

A.4 Inhibition kinetic parameters. . . . . . . . . . . . . . . . . . 140

A.5 Kinase and Phosphatase kinetic parameter values. . . . . . . . . . . 141

A.6 Parameter ranges for sensitivity analysis. . . . . . . . . . . . . . 141

B.1 Cav1.2 Phosphorylation Parameters . . . . . . . . . . . . . . . . . . . . . 144

B.2 Definition of model species, AKAP5 model . . . . . . . . . . . . . . . 144

B.3 PKA and AKAR Parameters . . . . . . . . . . . . . . . 147

B.4 PDE Parameters . . . . . . . . . . . . . . . . . . . . . . . . . . 148

B.5 AC Parameters . . . . . . . . . . . . . . . . . . . . . 150

B.6 Misc. Parameters . . . . . . . . . . . . . . . . . . . . . . . 152 


\title{
Abbreviations
}

\author{
Abbreviation Description \\ AC Adenylyl Cyclase \\ AKAP A-Kinase Anchoring Protein \\ ATP Adenosine Triphosphate \\ cAMP cyclic Adenosine Monophosphate \\ CaM Calmodulin \\ CaMKII $\mathrm{Ca}^{2+} /$ Calmodulin-Dependent Protein Kinase \\ CBP CREB-Binding Protein \\ CKAR C-Kinase Activity Reporter \\ CREB cAMP-Response Element Binding Protein \\ EGFR Epidermal Growth Factor Receptor \\ FRET Fluorescence Resonance Energy Transfer \\ FSK Forskolin, general AC activator \\ GAB GRB2-Associated Binding Protein \\ HBSS Hank's Balanced Salt Solution \\ I-1 Inhibitor 1 \\ ICUE Indicator of cAMP Using Epac \\ IQGAP IQ motif congaing GTPase \\ ISO Isoproterenol, $\beta$-adrenergic agonist \\ IBMX 3-isobutyl-1-methylxanthine,general PDE inhibitor
}




$\begin{array}{ll}\text { JNK } & \text { c-Jun N-terminal Kinase } \\ \text { LTCC } & \text { L-Type Calcium Channel } \\ \text { LQT } & \text { Long QT } \\ \text { MAPK } & \text { Mitogen-Activated Protein Kinase } \\ \text { MAPKK } & \text { MAPK Kinase } \\ \text { MAPKKK } & \text { MAPKK Kinase } \\ \text { NF } \kappa \text { B } & \text { Nuclear Factor kappa-light-chain-enhancer of Activated B Cells } \\ \text { NFAT } & \text { Nuclear Factor of Activated T cells } \\ \text { NRON } & \text { non-coding Repressor of NFAT } \\ \text { ODE } & \text { Ordinary Differential Equation } \\ \text { PDE } & \text { Phosphodiesterase } \\ \text { PDBu } & \text { Phorbol 12,13-dibutyrate, PKC activator } \\ \text { PI3K } & \text { Phosphoinositide 3-Kinase } \\ \text { PKA } & \text { Protein Kinase A } \\ \text { PKC } & \text { Protein Kinase C } \\ \text { PKD } & \text { Protein Kinase D } \\ \text { PKI } & \text { PKA Inhibitor } \\ \text { PLN } & \text { Protein phosphatase 1 } \\ \text { PP2A } & \text { Pab3-interacting Molecule } \\ \text { TEA } & \text { Pethylammonium chloride, Potassium Channel Inhibitor } \\ \text { PAmosphatase 2A }\end{array}$




\section{Chapter 1}

\section{Dissertation Aims}




\section{$1.1 \quad$ Introduction}

The basis of intracellular communication and information processing lies in cascades of chemical reactions catalyzed by enzymes. Many of the signaling proteins in these cascades have multiple downstream targets that lead to diverse or even contradictory cellular outcomes. These signaling proteins can be co-localized on scaffold proteins, which amplify and accelerate signal transduction. Additionally, this amplification and acceleration has been hypothesized to allow promiscuous signaling enzymes to have context dependent specificity. While scaffold proteins have been shown to be essential regulators of signal transduction, the underlying mechanisms of these scaffold derived emergent phenomenon are not understood. To address this gap in knowledge, we proposed the novel "scaffold state-switching" mechanism, where the enzyme-substrate-scaffold complex can stochastically switch between active and inactive intermediate states before the enzyme completes catalysis. In addition, these scaffold proteins often bind several interacting signaling proteins, creating complex signalosomes, and quantitative tools are needed to interpret and dissect the functional role of scaffold proteins. Through the combination of computational modeling and cellular imaging, we test the overall hypothesis that scaffold proteins modulate signal transduction by increasing the rate of enzyme-substrate interaction and coordinating crosstalk between signaling networks. Specific aims for this dissertation are:

\subsection{Aims}

Aim 1: Develop and validate a mechanistic computational model of scaffold state-switching kinetics. To evaluate the scaffold state-switching model, we developed computational models of PKC mediated phosphorylation both in solution and on the sim- 
ple scaffold, A-kinase Anchoring Protein $7 \alpha(\mathrm{AKAP} 7 \alpha)$. We experimentally validated this model using FRET biosensors for PKC activity targeted to the plasma membrane or linked to AKAP7 $\alpha$. Extension of these computational models to evaluate the effect of scaffold tethering on different inhibitors led to the prediction and subsequent validation that scaffold proteins can insulate the tethered enzyme from substrate- and ATP-competitive inhibitors but not activation-competitive inhibitors.

Aim 2: Test the hypothesis that AKAP5 is essential for the coordination of crosstalk between oscillatory Protein Kinase A and calcium signaling. Experimentally, we showed that both AKAP5 binding to Cav1.2 and AKAP-tethering of PKA are necessary for the development of regular $\mathrm{Ca}^{2+}$ oscillations in MIN6 cells. Furthermore, we observed that AKAP5 tethered PKA oscillates in-phase with calcium, in contrast with the out-of-phase PKA oscillations observed at the plasma membrane. We developed computational models exploring mechanisms underlying AKAP5 coordination of simultaneous inand out-of-phase PKA and calcium oscillations. These models predict that tethering of $\mathrm{Ca}^{2+}$ activated adenylyl cyclase and PKC enable AKAP5 to be responsible for the activation of two spatially and temporally separate pools of PKA. Additionally, this model was extended to show that AKAP5 is essential for the development of regular $\mathrm{Ca}^{2+}$ oscillations because it coordinates the positive feedback of PKA onto $\mathrm{Ca}_{\mathrm{V}} 1.2$.

These aims elucidate fundamental design principles governed by scaffold tethering and provide tools to interrogate the effects of scaffold proteins across a signaling network. Further, this work emphasizes the importance of scaffold proteins in cellular physiology and suggests the opportunity for targeting scaffold protein interactions for therapeutic gains. 


\section{Chapter 2}

\section{Background and Significance}

Reprinted from: E.C. Greenwald and J.J. Saucerman. "Bigger, Better, Faster: Principles and Models of AKAP Anchoring Protein Signaling." Journal of Cardiovascular Pharmacology Vol. 58 pp 462-469 (2011), with permission from Wolters Kluwer Health 


\section{$2.1 \quad$ Foreword}

Scaffold proteins constitute a broad and diverse class of proteins that adhere to the very general definition: they bind two or more signaling proteins. While these proteins may not actively transduce cell signals, the seemingly simple role of tethering proteins has been shown to create profound effects [1]. In this dissertation, we aim to study fundamental properties that underlie the effects of scaffold proteins. To focus our analysis of scaffold proteins, we utilize the A-Kinase Anchoring Protein (AKAP) family of scaffold proteins. AKAPs are a well-studied family of scaffold proteins and thus provide several opportunities to study the fundamental properties of scaffold tethered signaling [2]. In this chapter, we review the effects of scaffold tethering as seen for AKAPs, as a prototypical scaffold protein, and the use of computational models to identify how scaffold proteins effect signal transduction.

\subsection{Introduction}

A-kinase anchoring proteins (AKAPs) are a family of proteins that share the ability to bind the regulatory subunit of protein kinase A (PKA). AKAPs regulate a wide range of signaling molecules and cellular processes, binding both PKA and other partners simultaneously. But AKAPs generally do not directly mediate signal propagation. Instead, AKAPs act as scaffolds that modulate the signaling between constituent binding partners. AKAPs affect the location and dynamics of signal transduction by tethering specific signaling molecules to a particular location in the cell. Over the past several years, a wealth of molecular mechanisms underlying AKAP signaling has been revealed, much of which is described in the other reviews of this series on AKAPs [3] or reviewed previously elsewhere $[2,4,5]$.

In parallel with increasing molecular characterization, the role of AKAPs in physiology 
and disease has also been increasingly established. Thirteen different AKAPs have been identified in cardiac myocytes [6]. Several of these AKAPs have been shown to regulate of the inotropic, chronotropic and lusitropic state of the heart [7]. AKAPs have been shown to have important roles in pathological states such as cardiac arrhythmia $[8,9]$ cardiac hypertrophy [10] and familial breast cancer [11]. AKAPs are of particular interest as potential drug targets due to their specific, modulating role in systems governed by effectors that also have many other functions. While inhibiting a highly conserved kinase such as PKA would have widespread consequences in many tissues, AKAP-targeted therapies may allow tissue or even subcellular compartment-specific action. But the indirect signaling role that makes AKAPs attractive therapeutic targets also makes it difficult to assess AKAP function directly using traditional experimental approaches.

Computational modeling can aid understanding of complex networks where intuition alone is insufficient or misleading. Complementing experiments with computational models can allow one to identify key mechanisms underlying a biological response, predict the multiscale physiological consequences of these mechanisms, and perform in silico experiments that are not otherwise feasible [12]. Recent efforts have begun to include AKAPs and other scaffolds into models of signaling networks, helping to clarify the functional role of these scaffolds in cellular information processing. This review provides an overview of how computational models are being used to reveal fundamental principles by which AKAPs shape cellular signals.

\subsection{Design Principles for AKAP Function}

Given that AKAPs act as modifiers rather than direct transducers of cellular signaling, what are the design principles that govern their function? To answer this question, we must 
A

Localization

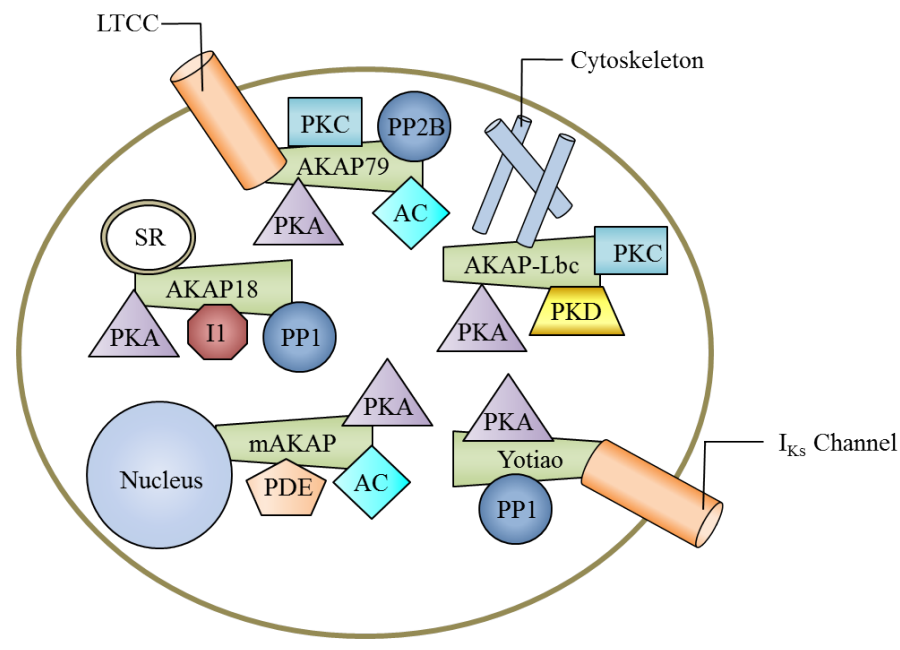

C

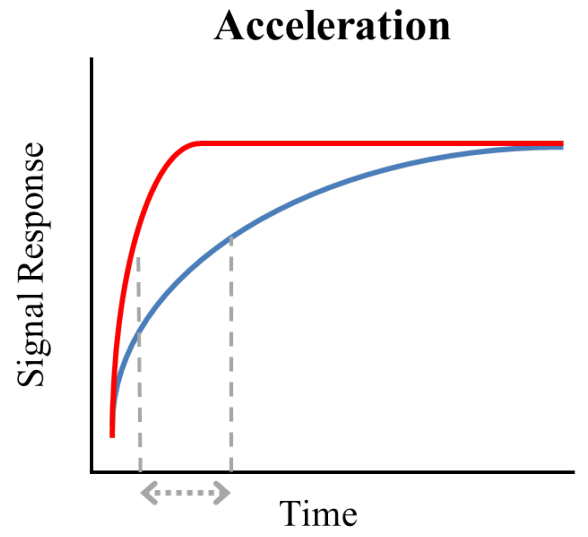

B

Specificity

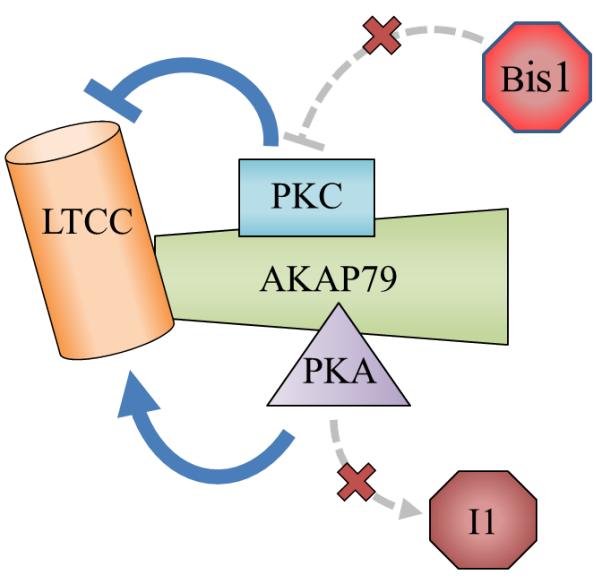

D

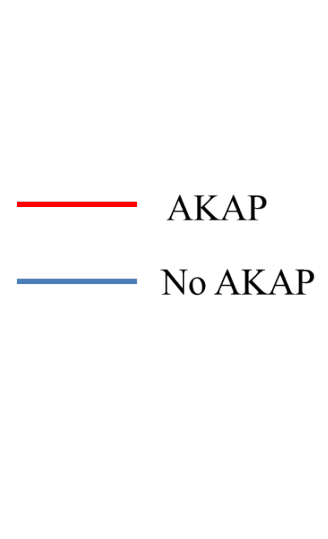

\section{Amplification}

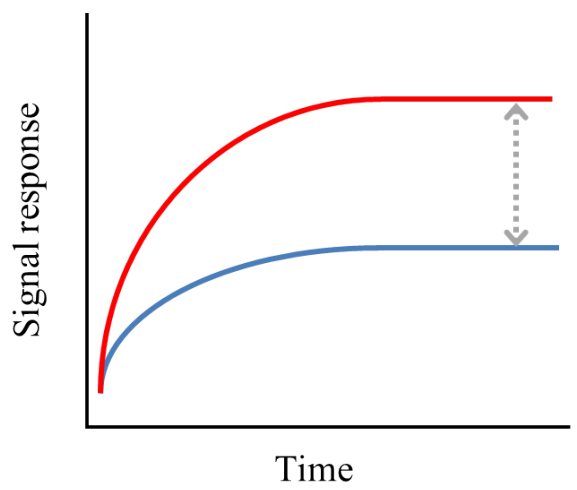

Figure 2.1: Design principles of AKAP signaling. A) AKAPs localize many signaling proteins to specific locations within the cell [13-16]. B) AKAPs can create preferential interactions on the scaffold. C) AKAPs can increase the rate at which signal transduction occurs. D) AKAPs can increase the magnitude of the signal response. AC- Adenylyl Cyclase, I1- Inhibitor 1, LTCC L-type Calcium Channel,PDE - Phosphodiesterase PKA - Protein Kinase A, PKC - Protein Kinase C, PKD - Protein Kinase D, PP1 - Protein Phosphatase, PP2B - Protein Phosphatase 2B, SR Sarcoplasmic Reticulum 
examine the fundamental biophysical mechanisms that enable AKAPs to modify signal flow through a pathway. Here, we outline four main design principles by which AKAPs have been shown to influence signaling: localization, specificity, amplification, and acceleration (Figure 2.1).

AKAPs have the ability to localize their binding partners to specific physical domains within the cell. Many AKAPs have targeting domains that anchor them to various subcellular compartments such as the plasma membrane, mitochondria, endoplasmic reticulum, centrosome, and nuclear membrane (Figure 2.1A) [13-16]. It is not a requirement that AKAPs have a targeting domain, but most AKAPs have been shown to contain localization capabilities $[13,17]$. Targeting to specific subcellular compartments helps mediate signaling that is specific to that compartment. For example, AKAP-Lbc localizes its protein complex to the cytoskeleton, allowing it to direct cell migration, influence PKA activity gradients at the plasma membrane, and regulate cardiac myocyte hypertrophy $[10,18]$. AKAP localization can also be dynamic, with post-translational modifications causing AKAP translocation in response to a cellular signal. This is evident through reversible palmitoylation that targets AKAP18 to the plasma membrane and the dynamic recruitment of PKA to mitochondria during oocyte maturation $[14,19]$.

AKAPs have the ability to bring not only PKA but multiple interacting partners together to create a separate signaling nanodomain termed a "signalosome" [5, 20]. Indeed, a large number of proteins have been shown to bind to AKAPs [2]. By defining which proteins are in a particular signalosome, the AKAP may able to enhance signaling pathways within the scaffold while minimizing the spread of signaling outside the scaffold, creating response specificity (Figure 2.1B). This may be especially important for AKAPs due to the fact that PKA may have $>250$ protein substrates $[21,22]$. It has been hypothesized that scaffolds were evolutionarily selected because they allow the cell to maintain fewer signaling proteins while 
still ensuring specificity [23]. Indeed, protein scaffolds appear to be early adopters of the mantra "reduce, reuse, recycle" as they reconfigure existing components for new uses. One example of bringing interacting proteins together is mAKAP, which binds PKA, phosphodiesterase PDE4D3, adenylyl cyclase-5 (AC5) and others [24,25]. This increased proximity helps PKA activate the PDE and negatively feedback on AC5, down-regulating cAMP. These particular interactions have important physiological consequences for cardiovascular physiology, as disrupting the binding of mAKAP to AC5 caused an increase in global cAMP levels and myocyte hypertrophy [25]. In perhaps the clearest example of AKAP specificity to date, Hoshi et al. found that AKAP79 not only enhanced interactions between PKC and its substrates but also prevented interactions with certain molecules not on the AKAP [26]. While a noncompetitive PKC inhibitor was unaffected by AKAP-PKC interactions, AKAP-bound PKC was insensitive to the competitive PKC inhibitor BIS-1 [26]. This raises an interesting challenge for drug target selection as AKAP-induced specificity may alter the pharmacology of its binding partners.

In addition to arranging specific signaling proteins in new configurations, AKAPs may accelerate signal transduction by positioning the enzyme and substrate in close proximity (Figure 2.1C). The acceleration of downstream activation was clearly shown by Zhang et al. through the development of a fluorescence resonance energy transfer (FRET)-based Akinase activity reporter (AKAR) [27]. They used targeting sequences derived from AKAPs to localize PKA to the AKAR reporter and showed that with rapid cAMP stimulation, the PKA phosphorylated its substrate 10-fold faster than without tethering [27]. Similarly, Hoshi et al. found that fusing AKAP79 to a C Kinase Activity Reporter, CKAR, increased the rate of response to muscarinic stimulation to equal the response time of the endogenous KCNQ2 channel [26].

AKAPs also have the ability to amplify the signal response (Figure 2.1D). For exam- 
ple, AKAP75 amplifies PKA activity in the nucleus, which can increase the activation of transcription factors and subsequently amplify the expression of proteins such as cyclindependent kinase-2 inhibitor p2 $7^{\text {kip1 }}[28,29]$. This amplification is presumed to be due to localization of AKAP75 to the nuclear membrane to increase the rate of nuclear transport. Another way that AKAPs may amplify a signal is through dynamic regulation of substrate binding affinity. For example, AKAP-Lbc amplifies PKD activation by PKC and is involved in PKA regulation to further amplify PKD signaling [30]. It is hypothesized that the amplification by PKA is due to phosphorylation of AKAP-Lbc by PKA, which reduces the binding affinity of PKD and thus allows greater turnover of PKD and amplification of PKD signaling [30].

The ability of scaffolds to accelerate and amplify cell signals appears closely linked. Indeed, in a classic example of the drosophila phototransduction cascade, mutants of the scaffolding protein InaD decreased both response magnitudes and response times to light [31]. The signal acceleration and amplification by AKAPs has been hypothesized to be a result of the increase of the local concentrations of the enzyme and its intended target [32]. In a rough order-of-magnitude approximation Zeke et al. calculated that the "effective concentration" of a protein on a scaffold could be up to 3000 times greater than in solution [23]. Indeed, for a single molecule tethered within a $10 \mathrm{~nm}$ radius sphere, its effective concentration would be approximately $400 \mu \mathrm{M}$. Experimental evidence that AKAPs increase the effective concentration of an effector was shown by Tavalin et al. where introduction of AKAP79 caused a 4-fold acceleration and a 20-fold shift in the concentration of PKC needed to regulate GluR1 receptor currents [33]. These responses are similar to the 10-fold acceleration of PKA-mediated phosphorylation shown by Zhang et al. [27], but the quantitative extent to which "effective concentration" is enhanced may be different for each AKAP. Work done by Persechini et al. on calmodulin provides additional direct experimental evidence of the 
effective concentration hypothesis [34]. Calmodulin mimics a scaffold because it contains two distinct Ca-binding lobes that both interact with target proteins, thus acting like two separate regulators tethered together. By titrating calmodulin fragments containing just one lobe or the other, they were able to show that once one calmodulin lobe binds to a target protein, the effective concentration of the second lobe is approximately $1 \mathrm{mM}$ [34].

The ability of AKAPs to localize, specify, accelerate and amplify biochemical signals are important design principles for understanding the information processing provided by cell signaling networks. Characterizing these principles experimentally requires quantitative experimental approaches such as the fluorescent reporters and electrophysiology described above. These experiments will provide the necessary ingredients for computational models that enable a quantitative understanding of the relationships between biophysical mechanisms and physiological consequences of AKAP signaling.

\subsection{Network Motifs on AKAP Signaling Complexes}

Although the four design principles discussed above focus on how AKAPs modulate the interaction between two proteins, additional functional properties are revealed when we begin to examine higher-order interactions occurring on a scaffold. Several systems concepts useful for understanding AKAP signaling are defined in Table 2.1. Recurring patterns of interactions called network motifs form the building blocks of complex networks [35]. Common network motifs such as feedback, feedforward, and bifan structures have been shown to be statistically enriched across many types of networks, from internet structure and social networks to microbial transcription [35]. By recruiting specific binding partners to the scaffold, there is increasing evidence that AKAPs form network motifs that may have important functional consequences for their cellular signaling. Network motifs found on AKAPs have been shown 
to cause characteristic signal response behaviors such as adaptation [24], oscillation [36] and ultrasensitivity [37].

One common signaling response behavior is adaptation, defined as the ability of a network to respond to a sustained input but then return to the pre-stimulated state (Figure 2.2A) [38]. This is important to be able to sense the change as opposed to the magnitude of a stimulus. Ma et al. computationally tested all possible interactions between three signaling proteins and found just two basic motifs allowing adaptation: negative feedback and incoherent feed forward loops [38]. Negative feedback occurs when a "downstream" protein inhibits an "upstream" protein, while incoherent feedforward loops are formed when a particular protein activates and then later inhibits the same protein. For example, mAKAP organizes a negative feedback motif where PKA phosphorylates PDE4, which degrades cAMP and results in decreased PKA activity, see Figure 2.2A [24]. Dodge-Kafka et al. built a synthetic AKAP complex incorporating the PKA reporter AKAR, PKA and PDE, which exhibited adaptive PKA activity in response to stimuli, whereas complexes without PDE binding did not [24]. Adaptive responses exhibit either a single overshoot (shown in Figure 2.2A) or damped oscillations, depending on the nonlinearity and time constants involved as shown clearly for the $\mathrm{NF} \kappa \mathrm{B}$ system [39]. While feedback and feedforward motifs can drive adaptation, their existence in a network does not guarantee that adaptation will occur.

Oscillation is another physiologically important signaling response which has been shown to be caused primarily through negative feedback loops (Figure 2.2B) [40]. One special requirement that separates this response from adaptation is that the feedback must be sufficiently delayed [40]. AKAPs have been shown to affect the frequency and amplitude of estradiol-induced $\mathrm{Ca}^{2+}$ oscillations in neurons [36]. This was hypothesized to be due to the AKAP's regulation of the L-type Calcium Channel (LTCC) and possibly other downstream effectors of scaffold-bound PKC such as MAPK and PI3K [36]. Another important physi- 
ological example of oscillation is pulsatile insulin secretion in the pancreas, which involves oscillatory $\mathrm{Ca}^{2+}$-triggered exocytosis [21]. Ni et al. showed MIN6 cells exhibited $\mathrm{Ca}^{2+}$ oscillations whose amplitude and magnitude are regulated by PKA activity [21]. This oscillatory circuit is hypothesized to be formed by PKA potentiation of the LTCC, resulting in $\mathrm{Ca}^{2+}$ dependent negative feedback on cAMP, either through PDE or AC (see Figure 2.2B) [21]. This finding of direct PKA regulation of $\mathrm{Ca}^{2+}$ oscillation may implicate a coordination role of AKAPs in this process because PKA, LTCC and AC5/AC6 associate with AKAP79 in these cells $[32,41,42]$. It is important to mention that even though AKAPs can enhance oscillations, it may also be possible for AKAPs to diminish oscillations by reducing the delay in the negative feedback of the network through acceleration.

Ultrasensitivity is a switch-like response to increasing input, characterized by an increased apparent Hill coefficient (Figure 2.2C) [43]. Ultrasensitivity is important because it allows the system to be activated quickly or allow the network to filter out weak signal noise [44]. Ultrasensitivity has been shown to have four underlying mechanisms: cooperativity, multistep, zero-order, and inhibitor $[43,44]$. The classic example of ultrasensitivity is seen in the cooperative oxygen binding to hemoglobin. Multistep ultrasensitivity arises when a signaling effector has multiple feed-forward interactions [43]. For example, MAPK proteins require two distinct phosphorylations by a single upstream kinase for activation which creates multistep ultrasensitivity [44]. Zero-order ultrasensitivity occurs when enzymes are operating at near saturation levels, which occurs when the substrate concentration is relatively large, compared to the Michaelis constant [43]. It is possible that AKAPs may create zero-order sensitivity by increasing the effective local concentration of the substrate to saturating levels. Finally, it has been shown that the presence of a stoichiometric inhibitor can cause ultrasensitivity because at low stimulation levels the enzyme is blocked by the inhibitor [44]. For example, PKI, a PKA inhibitor, was predicted to enhance the ultrasensitivity of cAMP-dependent 
PKA activation [45].

Ultrasensitivity may result from a combination of the above mechanisms and other network motifs on AKAPs. For example, Mutalik and Venkatesh used a computational model to show that incorporation of Inhibitor-1 (I-1) in the signaling cascade may greatly increase the sensitivity of many downstream effectors [37]. The system studied by Mutalik and Venkatesh had already been shown to exhibit zero-order ultrasensitivity but the increased sensitivity was hypothesized as being due to feedforward actions of PKA and the PP1 inhibition by I-1 (see Figure 2.2C). While that study was not focused on AKAPs, others have shown that that AKAP18 binds PKA, I-1 and PP1 and that I-1 phosphorylation by PKA is increased by I-1 binding to the scaffold [46]. By bringing these three components together on an AKAP, it may be possible to create an ultrasensitive signaling response.

AKAPs may enable network responses such as adaptation, oscillation and ultrasensitivity, by organizing the required network motifs efficiently. This may allow them to localize these signal responses to a specific area and provide a compartment specific response to a signal. 


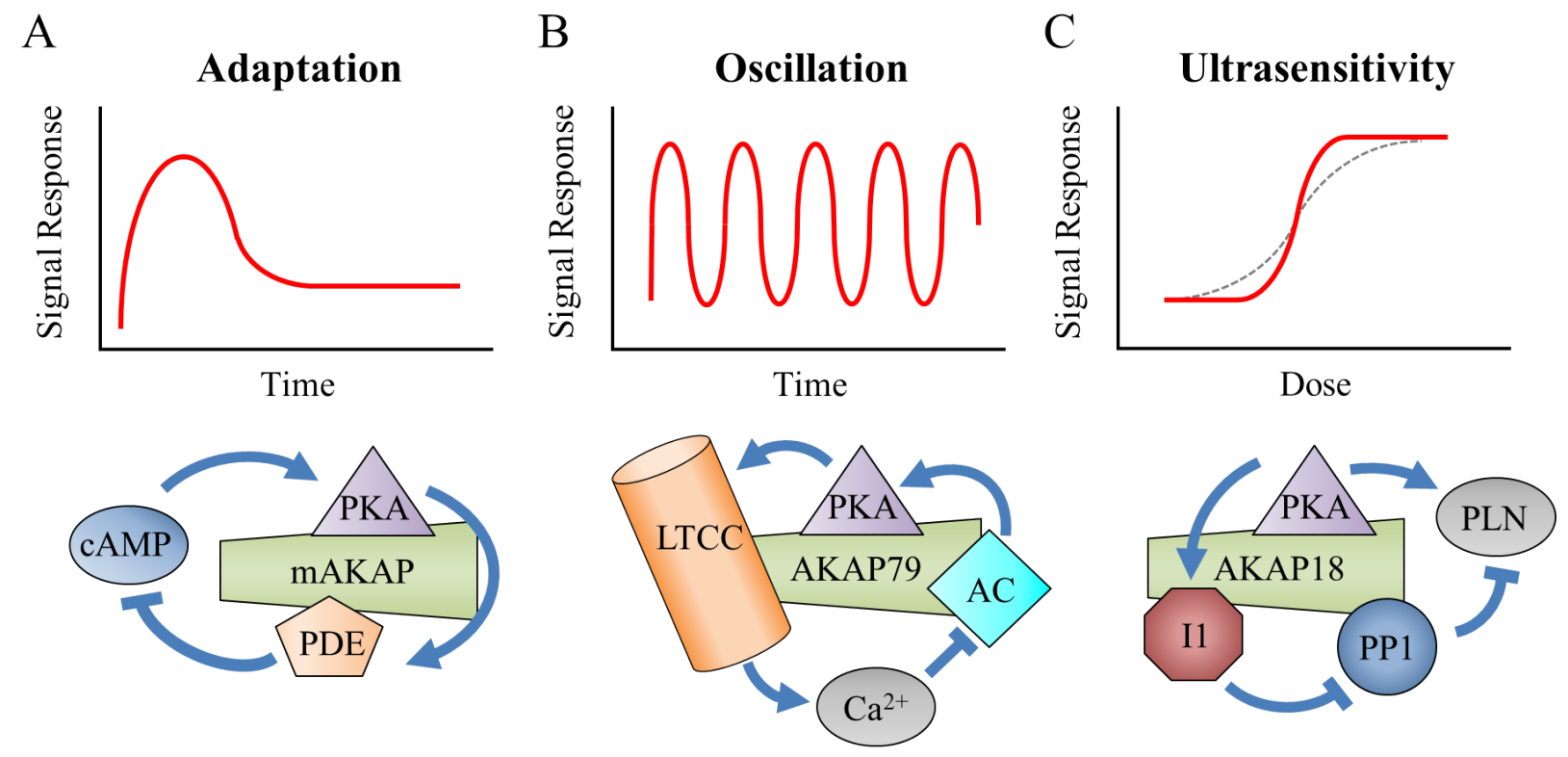

Figure 2.2: Examples of complex signaling responses and corresponding network motifs that are possible on AKAPs. A) The negative feedback loop through PDE can create signal adaptation [24]. B) Calcium negative feedback with significant delay can create oscillation in PKA activity [21]. C) The dual actions of PKA on Phospholamban (PLN) and the phosphatase inhibitor I1 can both increase the Hill coefficient of the signal response [46]. 
Table 2.1: Key Systems Concepts Relevant to Understanding AKAP Signaling

\begin{tabular}{|cc|}
\hline \multicolumn{1}{|c|}{ Term } & \multicolumn{1}{c|}{ Definition and References } \\
\hline Design Principles & Fundamental ways in which AKAPs shape cellular signals \\
Localization & Tethering of a protein to a physical subcellular location [13-17,47] \\
Specificity & Preferential interaction of proteins bound to an AKAP [24-26] \\
Acceleration & Increase in the speed of response to a stimulus [26,27,31] \\
Amplification & Increase in the magnitude of response to a stimulus [28-31,33] \\
& \\
Network Motifs & Topological patterns in signaling cascades [35] \\
Feedback & "Downstream" protein acting on an "upstream" protein [21,24,35,38] \\
Feedforward & "Upstream" protein affecting a "downstream" protein through two \\
& separate pathways [35,38,46] \\
Network Responses & System behaviors not attributable to any individual component \\
Adaptation & Return of a cellular signal to its pre-stimulated state while under \\
& continued stimulation [38] \\
Oscillation & Repeated fluctuations of a cellular signal [21,36,40] \\
Ultrasensitivity & Switch-like response to increasing input signal, exhibiting a dose re- \\
& sponse curve with Hill coefficient >1 [37,43,44] \\
& \\
\hline
\end{tabular}

\subsection{Computational Models of AKAP Signaling}

Computational modeling of signaling networks with scaffolds has proved useful in understanding how protein scaffolds integrate the effects seen at the protein interaction and signaling network levels. To do this one must consider the above design principles and determine how to represent these mathematically. Building a computational model of a signaling network requires a specific list of the biochemical reactions with stoichiometry, rate constants for each reaction (generally determined from prior biochemical experiments) and protein expression levels. When rate constants or expression levels have not been measured directly, these parameters may in some cases be inferred by fitting the model to quantitative 
timecourse data. A detailed introduction to these modeling approaches has been described previously [48]. Here we review computational models that have incorporated AKAPs into signaling networks as well as several models of other relevant protein scaffolds.

The first computational model to incorporate an AKAP into a signaling network was developed by Saucerman et al. [45]. This kinetic model examined how the $\beta$-adrenergic signaling pathway regulates excitation-contraction coupling in cardiac myocytes. While there are many components in this large kinetic model, one subtlety is the inclusion of an AKAP that localizes PKA, PP1, and PP2A to the L-type calcium channel (LTCC). The inclusion of this AKAP was necessary to be able to achieve the dynamic range of LTCC phosphorylation in response to $\beta$-adrenergic agonists seen experimentally. It was hypothesized that the AKAP accelerates PKA-mediated phosphorylation by increasing the effective concentration of the AKAP-bound LTCC [45]. This was achieved mathematically by multiplying scaffold-bound proteins by a 10-fold increase in effective local concentration, based on the acceleration of PKA-mediated phosphorylation seen experimentally when PKA was tethered to a fluorescent reporter $[27,45]$.

The necessity of the AKAP in this model emphasized the importance of AKAP scaffolding in signaling dynamics. But while an "effective concentration" AKAP model was sufficient to explain the experimental data in this case, there are several limitations that should be considered. First, the use of an effective concentration is somewhat phenomenological, because it does not mechanistically represent the interactions within the scaffold. Further, the effective concentration used was based on a recombinant fluorescent reporter rather than the endogenous LTCC, because such data was not available. It is likely that the degree of acceleration will vary both from AKAP to AKAP and between proteins on a given AKAP. Therefore it will be important to obtain more specific experimental data on a case-by-case basis and better characterize the biophysical determinants of signal acceleration. 
This computational model of $\beta$-adrenergic signaling was subsequently extended to examine how a mutation in the IKs channel causes Long QT (LQT) syndrome and arrhythmias during sympathetic stimulation [49]. This study was motivated by a clinically-observed G589D mutation in the KCNQ1 gene that was shown to disrupt the binding of an AKAP, yotiao, to the IKs channel [9]. Yotiao anchors PKA and PP1 to the IKs channel; by disrupting this interaction the mutation prevents PKA-mediated phosphorylation of IKs [9]. Despite these advances in molecular biology, the sequence of physiological mechanisms linking molecular interactions to clinical phenotype could not be addressed directly using genetic perturbations due the minimal role for IKs in mouse electrophysiology. Saucerman et al. incorporated these signaling mechanisms into the $\beta$-adrenergic signaling model discussed above, again using an "effective concentration" model to simulate reactions within the yotiao/IKs channel complex [49]. But in this case, increasing mechanistic detail was used to represent the reversible binding of yotiao to KCNQ1 and the reversible binding of PKA and PP1 to yotiao. By including this detail, they were able to further model the effect of the KCNQ1 mutation as a decreased affinity of yotiao for KCNQ1. The model showed that the disruption of the yotiao/KCNQ1 interface prevented $\beta$-adrenergic regulation of IKs seen experimentally, and was able to further predict increased action potential duration and early afterdepolarizations in myocytes along with LQT and increased transmural dispersion of repolarization in the ventricular wall. Thus, the computational model was able to provide a mechanistic and multi-scale explanation of how the KCNQ1-G589D gene mutation leads to a clinically relevant phenotype. Indeed, a subsequent clinical case study of a LQT1 patient demonstrated remarkably similar "Himalayan" T-waves to those predicted by the model [50].

Others have recently explored alternative mathematical approaches to modeling AKAP scaffolds. Andrei and Calder developed a stochastic reward-based analysis method for modeling interactions occurring on and off a scaffold [51]. They used this approach to develop a 
semi-quantitative model with interactions between cAMP, PKA, PDE and Raf on an AKAP, predicting "pulsations" of signaling. While such behaviors have not been reported experimentally, FRET reporters incorporating a cAMP-PKA-PDE negative feedback loop have shown adaptation in PKA activity [24].

\subsection{Computational Models of Other Signaling Scaffolds}

There have been a number of modeling studies of other scaffolds that are relevant to our understanding of AKAPs. Many of these examine MAPK scaffolds and GAB scaffolds that are associated with EGFR signaling. The focus here is to highlight the assumptions and methods that have been applied to model these scaffolds and the design principles of scaffold signaling elucidated by computational modeling.

Levchenko et al. developed a computational model to examine how scaffold concentration affects the acceleration and amplification of MAPK signaling [52]. To do this, they examined a two-member scaffold that binds both a MAPKK and its substrate MAPK. This model allowed for MAPK phosphorylation both in solution and on the scaffold, but kinases in solution were assumed not to phosphorylate MAPK on the scaffold. In contrast to the "effective concentration" AKAP model described above, Levchenko assumed that phosphorylation on the scaffold occurred at a maximal catalytic rate independent of the Michaelis constant Km. MAPK activation generally requires phosphorylation at two distinct sites and require two distinct enzyme interactions (dissociative), which as discussed above can create ultrasensitivity. But in this model it was assumed that both phosphorylation reactions on the scaffold occur simultaneously.

With these assumptions, Levchenko et al. showed that moderate concentrations of scaffold can both accelerate and amplify the signal response [52]. However, at high scaffold 
concentrations there was little or no amplification, forming a biphasic dependence on scaffold. The surprising prediction of decreased signaling at high scaffold concentrations was due to increasing the number of partially-filled scaffolds where MAPKK or MAPK proteins were isolated and inactive [52]. This suggested that cells may be able to modulate signaling pathways through changes in scaffold expression. This model also suggested that by rewiring sequential phosphorylation events to be simultaneous, the MAPK scaffold may diminish ultrasensitivity [43]. However, later experiments showed that MAPK scaffold signaling was still ultrasensitive [53].

O'Shaughnessy et al. investigated the properties of MAPK scaffold signaling further by combining synthetic biology and computational modeling [53]. They expressed the RafMEK-Erk cascade in yeast, along with varying expression levels of the MAPK scaffold Pax. Like the model predictions of Levchenko et al., they found that high scaffold expression levels diminish signaling by increasing the number of partially-filled scaffolds. But in contrast to the predictions of Levchenko et al., moderate concentrations of scaffold did not enhance signaling [53]. O'Shaughnessy developed a computational model of this system and showed that the lack of amplification by the scaffold may be because this particular MAPK cascade is inherently catalytically efficient when in solution [53]. These experimental and model results indicate that the effects of scaffolding may depend on the kinetic rate constants and concentrations for the particular system of interest.

Another model examining MAPK scaffold dynamics was developed by Locasale et al., which used stochastic spatial simulations to analyze how scaffolds may either amplify or attenuate signal propagation [54]. Their simulations indicated a key balance between the diffusion rate of the kinase and the activity of the phosphatase that dictates whether a scaffold causes amplification or attenuation. While in general one would expect a scaffold to amplify a signal (as discussed above), their model predicted that in fact scaffolds may limit 
amplification by reducing the number of substrates accessible to a mobile kinase. On the other hand, if the opposing phosphatase activity is high, the kinase may require scaffolding in order to effectively phosphorylate its substrates, resulting in amplification. Like the results of Levchenko and O'Shaugnessy et al., these simulation results highlight that while the intuitions and design principles outlined above may be good rules of thumb, the functional impact of scaffolds will depend on a number of factors and will need to be studied quantitatively on a case-by-case basis. While many of the lessons from MAPK scaffolding may be helpful to understanding AKAPs, there are some key differences. One difference is that MAPK scaffolds process information in a linear manner (MAPKKK to MAPKK to MAPK), whereas AKAPs can bring together many different signals and contain much more complex network motifs [55].

Another system that has benefited significantly from computational modeling of scaffolds is endothelial growth factor (EGF) receptor signaling. Kiyatkin et al. combined experimental and computational approaches to investigate how the scaffold protein Grb2-associated binder (GAB1) regulates the balance of MAPK and PI3K pathways in response to EGF [56]. They developed a comprehensive computational model of this system and experimentally validated its predictions under multiple EGF doses, GAB1 suppression, and pharmacological inhibitors. Their models and experiments helped them identify how GAB1 acts to amplify the PI3K pathway and extend the duration of transient MAPK activity. In addition to the feedback and feedforward motifs involved, this network was particularly challenging to model due to the numerous simultaneous docking interactions. Their model included six binding sites on GAB1, 3 binding domains on Grb2 and both cytoplasmic or membrane targeted scaffolds, resulting in vast number of possible combinations of multi-protein complexes [56]. For example, when considering just EGF receptor dimerization and possible phosphorylation states and binding partners, without GAB there can be as many as 1128 different states. 
This combinatorial state explosion has also been observed in models of Fc $\epsilon$ RI signaling, where 354 distinct states and 3680 chemical reactions are possible $[57,58]$.

The large number of protein complex combinations would generally make these models both computationally expensive and prohibitively time consuming to implement correctly. But several groups have identified new model reduction approaches or numerical algorithms to manage this complexity. The model reduction approach in the Kiyatkin model was developed by Borisov et al., which by assuming independent binding collapses many of the states into groups called macrostates $[59,60]$. A strength of this approach is that often the macrostates correspond with experimental observables, such as the overall Gab1 phosphorylation level rather combinations of modifications that are not accessible experimentally. Another method to reduce the burden of combinatorial complexity was developed by Faeder et al., which automatically generates the equations for each state using a reduced number of variables [58]. This takes advantage of the fact that while there may be a large number of possible states, often the reactions that occur on the multi-protein complexes only depend on a few of the states of the complex and are the same across many different combinations. This allows the definition of reaction classes which are all defined by the same kinetic equation and rate constants. For example, a model of FceRI signaling was developed to have 15 reaction classes with 21 rate constants to describe the 354 states in contrast to implementing each of the 3680 chemical reactions individually [58]. Finally, Sneddon et al. developed stochastic modeling software, NFsim, which keeps track of every individual molecular complex and uses rules to describe state changes [61]. This approach becomes beneficial when the number of potential states is much larger than the number of individual molecular species in the simulation.

The modeling studies discussed above provide a number of insights into the design principles of signaling on scaffolds. Studies of MAPK signaling have shown how scaffolds can 
accelerate or amplify signaling by increasing the effective concentration within the complex. On the other hand, certain combinations of rate constants can cause the scaffold to attenuate signaling. Models of the Gab1 scaffold have examined the impact of localization and how scaffolds may recruit entire protein complexes to the plasma membrane. The Gab1 model also nicely illustrates how scaffolds may help establish network motifs such as feedback loops that shape signaling dynamics. While specificity has not been directly studied in these studies, the effects of specificity are often included by assuming no enzymatic activity between the scaffold and cytosol. While the described models have been deterministic, there is increasing recognition of the functional importance of biological noise [62]. Bhalla examined the differences between stochastic and deterministic modeling for four different signaling cascades, finding that the effects of noise begin to dominate at volumes approaching $10^{-15} \mathrm{~L}$ [63]. Localization by AKAPs creates compartments of this size and commonly stoichiometries of just 1 protein per complex, warranting further examination of how randomness affects AKAP signaling.

\subsection{Conclusions}

AKAPs play an important role in shaping cellular signals and regulating physiology and disease. Computational modeling is beginning to provide insights into the design principles fundamental to AKAP signaling function including localization, specificity, acceleration and amplification. AKAPs also help form a variety of feedback and feedforward network motifs, considerably increasing the diversity of signaling dynamics. Recent modeling efforts of MAPK and EGFR scaffolds provide additional examples of how scaffolds may regulate signaling networks. Mathematical models aid formulation of quantitative hypotheses that best explain experimental data and prediction of responses to perturbations that are not cur- 
rently feasible experimentally. In the future, models of AKAP signaling are also likely to be helpful in identifying novel drug targets for cardiovascular disorders including hypertrophy, arrhythmia, and heart failure. 


\section{Chapter 3}

\section{Integrating Fluorescent Biosensor}

\section{Data Using Computational Models}

Adapted from: E.C. Greenwald, R.K. Polanowska-Grabowska, J.J. Saucerman. "Integrating Fluorescent Biosensor Data Using Computational Models." Fluorescent Protein-Based Biosensors, Methods in Molecular Biology Vol. 1071 pp 227-248 (2014), with permission from Springer

Author contribution: E.C. Greenwald - Computational modeling, designed experiments, wrote the paper. R.K. Polanowska-Grabowska - Performed experiments, designed experiments, analyzed data, assisted in writing the paper.J.J. Saucerman - Designed experiments, assisted in writing the paper. 


\section{$3.1 \quad$ Foreword}

Cellular signaling can exhibit complex dynamics that are dependent on several interconnected signaling proteins and second messengers. This complexity can make experimental data difficult to interpret and understand. One useful tool to interpret and dissect these data are computational models [12]. As discussed in the previous chapter, computational models can quantitatively evaluate hypothesized signaling mechanisms as well as generate new hypotheses. To address the aims of this dissertation, our work utilizes the development and analysis of computational models in conjunction with live-cell, biosensor data. Therefore, knowledge of the development of computational models and the incorporation of experimental data is essential to understanding the data and conclusions of this dissertation. In this chapter, we provide a detailed tutorial on the process of model building, incorporation of biosensor data and generation of model hypotheses.

\subsection{Introduction}

Fluorescence microscopy of genetically encoded fluorescence resonance energy transfer (FRET)based biosensors is a powerful technique for understanding signaling kinetics in living cells. These biosensors allow researchers to visualize and quantify the spatiotemporal distribution of signaling molecules within the cell. A variety of FRET biosensors have been developed to directly detect changes in intracellular concentrations or activation of signaling molecules in real time [64]. It is often the goal to understand how these biochemical dynamics are modulated by the overall signaling network, particularly in response to pharmacologic or genetic perturbations. The complexity of signaling networks often hinders attempts to relate biosensor data directly to the molecular mechanisms underlying dynamic cell responses. 
Computational models allow integration of diverse biochemical and biosensor data into a common quantitative framework. These models can be used to quantitatively evaluate the plausibility of current hypotheses with existing data or to computationally generate new hypotheses that can be tested in the wet lab. This chapter provides experimental biologists an accessible tutorial on computational modeling, such that they may begin to analyze the system-level implications of their fluorescence-based biosensor data.

\subsection{Materials}

\section{Computational modeling software}

The computational modeling in this chapter was performed in The Virtual Cell Software (version 5.1 or greater), from the Center for Cell Analysis and Modeling (www.nrcam.uchc.edu).

\section{Cell culture}

Primary cultured rat neonatal cardiac myocytes. The cells are derived from neonatal hearts using the Neonatal Cardiomyocyte Isolation Kit (Cellutron cat\# nc-6031) and plated on 35 mm glass-bottom culture dishes (MatTek Cat\# P35GC-1.5-14-C) coated with SureCoat (Cellutron, Cat\# sc-9035) at $10^{6}$ cells per dish. The cells are cultured in cell culture media at $37^{\circ} \mathrm{C}$ and $5 \% \mathrm{CO}_{2}$.

\section{Cell Imaging}

Tyrode's buffer (155 mM NaCl, $5 \mathrm{mM} \mathrm{KCl}, 2 \mathrm{mM} \mathrm{CaCl} 2,1 \mathrm{mM} \mathrm{MgCl} 2,2 \mathrm{mM} \mathrm{NaH}_{2} \mathrm{PO}_{4}$, $10 \mathrm{mM}$ HEPES, and $10 \mathrm{mM}$ glucose, $\mathrm{pH}$ 7.2.) is used for imaging since it provides low autofluorescence and good pH stability in ambient conditions. All images were collected on 
a wide-field inverted microscope (Olympus IX81) with an UPlanSApo 10X/1.3 numerical aperture (NA) objective (Olympus) and 12-bit Hamamatsu digital C9300-21 digital camera (Hamamatsu Photonics, Bridgewater, NJ). Fluorescent protein excitation and emission collection were controlled by a Lambda DG4 excitation filter, (Sutter Instrument Company), Lambda 10-3 emission filter wheel changer with a Smart-Shutter (Sutter Instrument Company) and ECFP/YFP-ET filter set (Chroma Technology, Rockingham, VT). Images were collected using the Image acquisition software IP-LAB and analyzed in ImageJ.

\section{FRET Based Reporters for Imaging cAMP and PKA Dynamics}

ICUE (Indicator of cAMP accumulation Using Epac) shows changes in intracellular cAMP concentration. Binding of cAMP to this biosensor leads to a decrease of acceptor emission (IA) and an increase of donor fluorescence (ID) [65]. AKAR (A-Kinase Activity Reporter) monitors the activity of PKA by phosphorylation of PKA substrate. AKAR phosphorylation results in an increased acceptor emission (IA) and decreased donor emission (ID) [27,66].

\section{Transfection}

To monitor either cAMP accumulation or PKA activation, cells are transfected with 1.2 $\mu \mathrm{g}$ per dish of ICUE or AKAR DNA plasmid 24 - 48 hours prior FRET imaging, using Lipofectamine 2000 Kit (Lipofectamine 2000 Kit, Invitrogen) as per the manufacturer's instruction $^{1}$.

\section{Cell Treatment}

Forskolin $(50 \mu \mathrm{M})$, IBMX ( $100 \mu \mathrm{M})$ and isoproterenol $(1 \mu \mathrm{M})$.

\footnotetext{
${ }^{1}$ For primary cells, such as rat neonatal cardiac myocytes, transfection efficiency varies from 5 to $15 \%$.
} 


\subsection{Model Generation}

\section{Model Definition}

This chapter focuses on the generation of computational models that incorporate experimental data from FRET-based biosensors. The main goal of computational models is to help answer questions that cannot be directly answered using biosensor responses and intuition alone. Computational models provide a unified understanding of how a signaling pathway acts as a whole by integrating experimental information about the different aspects of the signaling cascade. There are many issues that models address, such as quantitatively examining the viability of a hypothesis or evaluating competing hypotheses for explaining an observed phenomenon. These models are often then probed to make predictions and generate testable hypotheses about particular biological responses, such as the action of drugs or gene silencing.

When building a computational model, the most important consideration is the biological question that will be addressed. The biological question determines the size and scope of the model needed and dictates the expected output of the model. As a prototypical signaling pathway, this chapter will examine the production of the second messenger cyclic adenosine monophosphate (cAMP) and the subsequent activation of cAMP-dependent Protein Kinase A (PKA). PKA participates in many diverse and integral signaling pathways and thus understanding the dynamics of PKA activation is important in analyzing these pathways $[45,67]$. An example of a biological question that could be asked about PKA activation might be: "What is the rate-limiting step for PKA activity in response to receptor stimulation: cAMP production or PKA activation?" This question defines the scope of the model by saying that the desired output is PKA activity and the model must include both cAMP production and 
PKA activation.

Now that the scope and objective of the model system have been specified by the biological question, the specifics of the signaling pathway must be defined. This is done by sifting through the information presented in literature, as well as databases, to define the most accurate signaling network [68-70]. The model considered here (Figure 3.1B) incorporates cAMP production by direct activation of adenylyl cyclase (AC) upon stimulation with forskolin (FSK) or stimulation of AC by $\beta$-adrenergic receptor agonist isoproterenol (ISO). After synthesis, cAMP is degraded by cAMP-specific phosphodiesterase (PDE). The cAMP biosensor ICUE is directly incorporated into this model, binding cAMP to form an ICUE-cAMP complex [65]. PKA holoenzyme consists of two catalytic and two regulatory subunits. Upon full activation by cAMP binding to the regulatory subunits, PKA's catalytic subunits dissociate and phosphorylate its downstream targets. In this simplified model, PKA is represented as having just one regulatory and one catalytic subunit, which switch from an inactive to active state by the binding of two cAMP molecules followed by the dissociation of the regulatory subunits from the catalytic subunits (RC, ARC, A2RC, A2R and PKAC in order from inactive to active catalytic subunits). PKA activity can be monitored by the FRET biosensor A-Kinase Activity Reporter (AKAR) [27,66]. Activated PKA catalytic subunits phosphorylate AKAR, inducing a conformational change in the reporter that generates FRET signal. Both the AKAR phosphorylation and subsequent FRET signal are reversed by protein phosphatases.

It is a good rule of thumb to start with the simplest model possible and then expand as needed to explain the observed data. When developing any model, assumptions must be made explicit when publishing or disseminating the model. For example, the model presented here makes the simplifying assumption that activation of $\mathrm{AC}$ by stimulation of the $\beta$-adrenergic receptor by isoproterenol (ISO) can be approximated as direct AC activation 
in place of explicitly modeling the receptors themselves and activity of $\mathrm{G}$ proteins. Another assumption is that PKA activation, which requires the binding of two cAMP molecules, is not affected by which site the cAMP binds to (i.e. binding order is independent). These assumptions will affect the model and will be discussed later.

The last piece of information needed to build the model is the kinetic rate constants. This can be one of the more difficult aspects of model building because it requires extensive literature mining. It is often useful to look at published computational models, as authors will typically publish the original literature sources for the kinetic parameters $[45,67,71]$. However, many parameters are unavailable in the literature. In this case, you can start by using order-of-magnitude estimates of that parameter [72]. These gaps in information will be filled later in this chapter when we use the experimental biosensor data to constrain the model and fit these parameters.

\section{Model Implementation in Virtual Cell}

The computational models discussed here are formulated mathematically as Ordinary Differential Equations (ODEs). ODE models can be implemented and computationally solved using numerous programming languages (e.g. MATLAB, $\mathrm{C}++$ ), but for this chapter we will use the user-friendly Virtual Cell software (http://vcell.org/) [73]. A key advantage of Virtual Cell is that the math is performed "behind the scenes", so the user can focus on the biochemical reactions and biology of interest. Virtual Cell is free to use and was developed by Les Loew and colleagues at the National Resource of Cell Analysis and Modeling to be a simple yet powerful tool to allow students and biologists with relatively little math background to perform computational modeling [73]. Virtual Cell also has advanced capabilities including stochastic and spatial modeling; however, this book chapter will fo- 
cus on its basic ODE modeling features. More detailed instruction on the use of Virtual Cell, as well as additional tutorials and resources, can be found on the Virtual Cell webpage (http://vcell.org). The following steps provide a tutorial walk-through for creating your own simple cAMP/PKA pathway model in Virtual Cell.

First, creating a model. After loading Virtual Cell, create a user account if you don't have one already. To create a new model, select File $>$ New $>$ Biomodel. Then, define compartments. Virtual Cell can incorporate signaling pathways that occur in separate cellular compartments. Compartments can be physical compartments within the cell, such as the nucleus or mitochondria, but they can also represent regions of the cell that are separated by diffusional barriers within the cell, such as the dyadic cleft which is separated from the cytosol in cardiac myocytes. To create a compartment, use the "Compartment Tool" (empty circle icon) in the "Structure Diagram" tab of the Physiology section of the model, and click in the graph area to create a compartment. If sub-compartments are needed within the cell, click the "Compartment Tool" inside the cell to create individual sub-compartments. Our example uses one compartment to represent the cell, where the inside of the cell has been labeled "cytosol" (Figure 3.1A). Compartments can be renamed using the Object Properties tab in the lower window.

Next, define model species. To define the proteins and second messengers, referred to as species, use the "Species Tool" (green circle icon) and click in the compartment in which they will be reacting. If implementing a multi-compartment model, species that can interchange between compartments must be defined in all relevant compartments. Figure 3.1A presents all of the needed species, shown as small circles, inside the cytosol compartment. Each "species" has been labeled to define what it represents. It is important to note that complexes between two reactants, such as cAMP bound ICUE (ICUE_cAMP), or different phosphorylation states, such as AKAR and phosphorylated AKAR (AKARp), need to be 
defined as separate species. Again, rename species using the Object Properties tab.

The final step in defining a model is to define reactions. In the "Reaction Diagram" tab of the Physiology section, all of the species should be present in their assigned compartment before connecting reactants to products. Reactants are connected to products using the " $R X$ connection tool". When this tool is selected, click on a reactant species and drag it to the product species. A line with arrows pointing from reactant to product and a yellow box in the middle should be created (Figure 3.1B). If multiple reactants are combining to form a product, each additional reactant can be added to the reaction by dragging from the reactant to the yellow box of the desired reaction. For example, to define the cAMP binding to the inactive PKA holoenzyme to form the intermediate "ARC", a reaction is dragged from cAMP to ARC and then another reaction line is dragged from $\mathrm{RC}$ to the newly formed yellow box. Similarly, if multiple products are formed in the reaction, click on the yellow box of the desired reaction and drag to the additional product. Finally, enzymes can be connected to the reaction that they catalyze using the "set a catalyst" tool and dragging from the enzyme to the yellow box of the reaction. Once all of the reactions are created, your "Reaction Diagram" should look similar to Figure 3.1B. 
A)

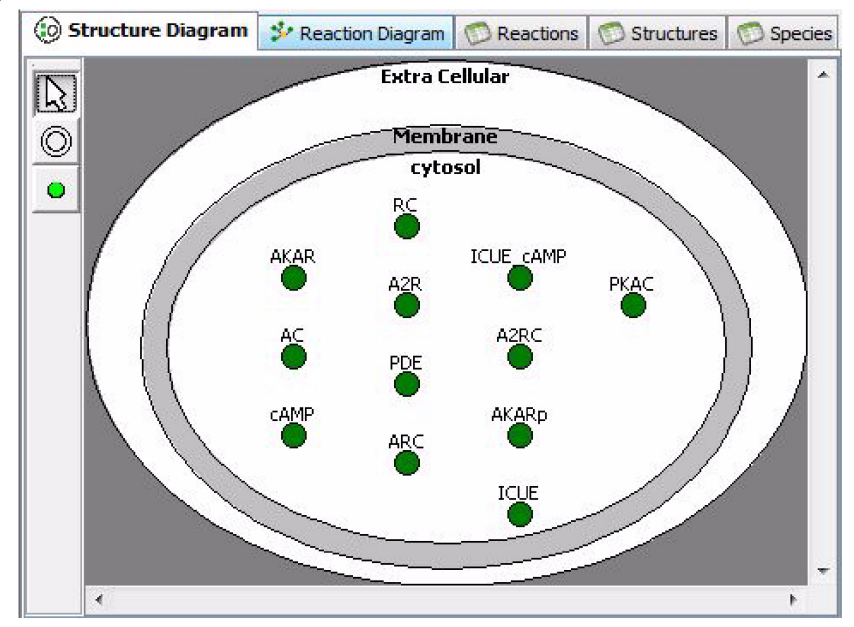

B)

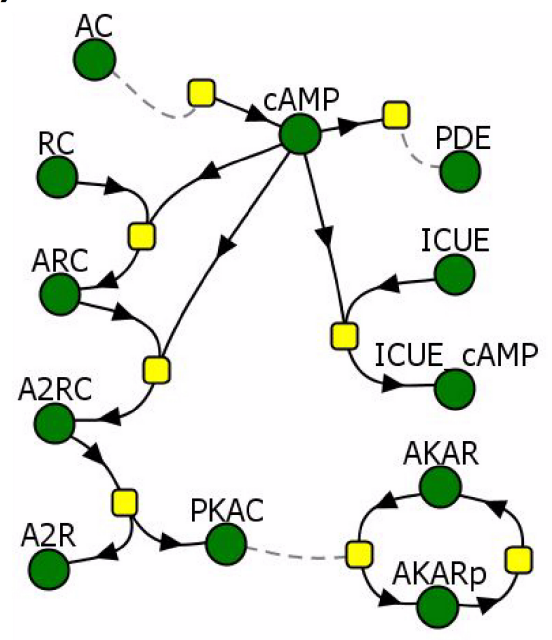

Figure 3.1: Virtual Cell Structure and Reaction Diagram: A) Virtual Cell structure diagram with the cell compartment and model species defined. B) Reaction diagram of model. Green circles represent model species, yellow boxes and arrowed lines are reactions and dotted lines represent enzyme catalysis. 
Once the Reaction Diagram is created, a kinetic type and rate constants must be defined for each of the reactions in the diagram. To define these, select the reaction (yellow box) and the equations and parameters for that reaction will be shown below ${ }^{2}$. The initial parameters used in our example model are listed in Table 3.1.

For binding or dissociation reactions, use the "Mass Action" kinetic type. Mass action kinetics allows the reaction to be reversible. Virtual Cell will then define the reaction rate equation based on how the species have been connected to it and create two variables, $K_{f}$ and $K_{r}$, which represent the forward and reverse rate constants, respectively. If the forward and reverse rate constants are not directly available in the literature, the dissociation constant, $K_{D}$, can be used to infer the rate constants using the following equation ${ }^{3}$.

$$
K_{D}=K_{r} / K_{f}
$$

An estimate of either $K_{r}$ or $K_{f}$ will allow for the calculation of the other constant such that its relative magnitude agrees with experimental data. The above equation can be rearranged and typed into the expression section for the associated rate constant and Virtual Cell will create a new variable, $K_{D}$, where you can input the dissociation constant value.

For enzyme catalyzed reactions, such as phosphorylation of AKAR (AKARp) by the catalytic subunit of PKA (PKAC), use the "Henri-Michaelis-Menten (Irreversible)" kinetic type. Virtual Cell will then create a reaction rate that has two variables, $K_{m}$ and $V_{\max } . K_{m}$ is the Michaelis constant and is often calculated when enzyme kinetics are measured. $V_{\max }$ is the "maximum velocity" of the enzyme catalysis, which can be described by $V_{\max }=k_{\text {cat }} * E_{\text {tot }}$,

\footnotetext{
${ }^{2}$ It is often useful to set kinetic parameters to have the value of 1 when setting up the model to be able to verify that each part of the model is working properly before taking the time to put specific values in for each parameter.

${ }^{3}$ The dissociation constant can sometimes be presented as the association constant, which is the inverse of $\mathrm{KD}$.
} 
Table 3.1: Initial parameter values defined for the example model. See public model "ecg5pc: Greenwald MIMB 2012 - Base Model" to relate reaction and parameter names to the model fluxes. Parameters that were used to fit the model to ICUE (Fit 1) or AKAR (Fit 2) data are identified by $\dagger$ and $\ddagger$, respectively.

\begin{tabular}{|c|c|c|c|}
\hline Reaction & Parameter & Value & Unit \\
\hline \multirow{11}{*}{ cAMP_synth } & ATP & 5000 & $\mu \mathrm{M}$ \\
\hline & $\mathrm{Km}$ & 860 & $\mu \mathrm{M}$ \\
\hline & Km_Iso & 315 & $\mu \mathrm{M}$ \\
\hline & kcat_iso† & 0.75 & $\mathrm{~s}^{-1}$ \\
\hline & Kd_iso & 0.1 & $\mu \mathrm{M}$ \\
\hline & kfsk† & 7.3 & $\mathrm{~S}^{-1}$ \\
\hline & IsO_stim & 1 & $\mu \mathrm{M}$ \\
\hline & t_iso & 200 & s \\
\hline & Kd_fsk & 860 & $\mu \mathrm{M}$ \\
\hline & FSK_stim & 50 & $\mu \mathrm{M}$ \\
\hline & t_FSK & 600 & S \\
\hline \multirow{5}{*}{ cAMP_deg } & $\mathrm{Km}$ & 1.305 & $\mu \mathrm{M}$ \\
\hline & kcat & 5 & $\mathrm{~s}^{-1}$ \\
\hline & $\mathrm{Ki}$ & 30 & $\mu \mathrm{M}$ \\
\hline & IBMX_stim & 100 & $\mu \mathrm{M}$ \\
\hline & t_IBMX & 600 & $\mathrm{~s}$ \\
\hline \multirow{2}{*}{ ICUE_bind } & $\mathrm{Kf} \dagger$ & 5 & $\mu \mathrm{M}^{-1} \mathrm{~s}^{-1}$ \\
\hline & $\mathrm{Kr}$ & 10 & $\mathrm{~s}^{-1}$ \\
\hline \multirow{2}{*}{ PKA_bind1 } & $\mathrm{Kf} \ddagger$ & 1000 & $\mu \mathrm{M}^{-1} \mathrm{~s}^{-1}$ \\
\hline & $\mathrm{Kr} \ddagger$ & 9140 & $\mathrm{~s}^{-1}$ \\
\hline \multirow{2}{*}{ PKA_bind2 } & $\mathrm{Kff}$ & 1000 & $\mu \mathrm{M}^{-1} \mathrm{~s}^{-1}$ \\
\hline & $\mathrm{Kr}$ & 1640 & $\mathrm{~s}^{-1}$ \\
\hline \multirow{2}{*}{ PKA_act } & $\mathrm{Kf}$ & 4375 & $\mathrm{~s}^{-1}$ \\
\hline & $\mathrm{Kr}$ & 1000 & $\mu \mathrm{M}^{-1} \mathrm{~s}^{-1}$ \\
\hline \multirow{2}{*}{ AKAR_phos } & $\mathrm{Km} \neq$ & 21 & $\mu \mathrm{M}$ \\
\hline & kcat & 54 & $\mathrm{~s}^{-1}$ \\
\hline \multirow{2}{*}{ AKAR_dephos } & kcatf & 8.5 & $\mu \mathrm{M}^{-1} \mathrm{~s}^{-1}$ \\
\hline & PPasef & 2.14 & $\mu \mathrm{M}$ \\
\hline
\end{tabular}


where $k_{c a t}$ is the catalytic rate constant and $E_{t o t}$ is the concentration of the active enzyme. Put this equation into the expression column for $V_{\max }$ and in place of $E_{\text {tot }}$ put the species name given to the relevant enzyme that catalyzes this reaction, e.g. PKAC for AKAR phosphorylation. Virtual Cell will automatically create a new variable for $k_{c a t}$ where you can define the catalytic rate constant for this reaction. For enzymatic reactions that do not have an explicit reactant defined (e.g. production of cAMP), Virtual Cell is not able to automatically define the Michaelis-Menten rate equation. Therefore, for this reaction, you will need to choose the general kinetic type and manually input the Michaelis-Menten reaction rate expression.

Finally, for reactions that do not fall into these two categories, the "General kinetic type", where the user defines the equation for the reaction rate manually, can be used. One common expression used in models is the Michaelis Type equation, which has the following general form:

$$
\frac{A}{E C_{50}+A}
$$

where $A$ is the species of interest and $E C_{50}$ is the concentration of species $\mathrm{A}$ at which half of the maximal activity is achieved. This form of the equation is used in our model to approximate ISO stimulation of cAMP production.

Public models can be accessed by selecting file>open $>$ biomodel and selecting the model of interest from the Public Biomodels folder. Use our public Virtual Cell model (model name "ecg5pc: Greenwald MIMB 2012 - Base Model") to verify that the reactions and rate parameters are specified correctly in your model. In particular, examine the cAMP synthesis reaction because this reaction required manual definition and incorporates Michaelis type equations to define stimulus activation strength.

Now that the model has been defined in Virtual Cell, the behavior of the model is de- 
termined by numerical solution of the model equations. To accomplish this, first create a new application by right clicking on "Applications", selecting "Add New" and select "Deterministic". This will create a new application to numerically solve the ODE model you defined in Virtual Cell. If creating a multi-compartment model, you will need to specify the volume of each compartment. If using a single compartment, as is used in the example, it is usually not necessary to change the size definitions away from the default. Next, define parameters for each of the species in the model. Under the "Specifications" tab will be a list of all the species in the model. This is where you define the initial concentrations of each species under the "Initial Condition" column. The initial concentrations used in our model are defined in Table 3.2. It is also possible in this section to specify a species as having a fixed concentration over time by checking the box under the "Clamped" column. For example, ATP concentration is often assumed to be constant because of its high concentration and strict regulation within the cell.

Table 3.2: Initial concentrations used in the example model. $\ddagger$ identifies parameters that were allowed to vary to fit AKAR data (Fit 2).

\begin{tabular}{|lrr|}
\hline Species & \multicolumn{2}{c|}{ Conc. } \\
\hline cAMP & 0 & $\mu \mathrm{M}$ \\
AKAR & 1.25 & $\mu \mathrm{M}$ \\
AC & 0.05 & $\mu \mathrm{M}$ \\
RC $\ddagger$ & 1 & $\mu \mathrm{M}$ \\
A2R & 0 & $\mu \mathrm{M}$ \\
PDE & 0.014 & $\mu \mathrm{M}$ \\
ARC & 0 & $\mu \mathrm{M}$ \\
ICUE_cAMP & 0 & $\mu \mathrm{M}$ \\
A2RC & 0 & $\mu \mathrm{M}$ \\
AKARp & 0 & $\mu \mathrm{M}$ \\
ICUE & 0.15 & $\mu \mathrm{M}$ \\
PKAC & 0 & $\mu \mathrm{M}$ \\
\hline
\end{tabular}


You are now ready to run the simulation. Under the "Simulations" tab, click the "New Simulation" button. Select the simulation that was just created and push the "Edit Simulation" button. From this window you can specify what changes you wish to make for this simulation. This can be used to simulate the addition of a stimulus, such as changing the concentration of ISO from 0 to $1 \mu \mathrm{M}$ at the start of the simulation which is done by entering the value $1 \mu \mathrm{M}$ in the "New Value/Expression" column for ISO. It is often necessary to be able to apply a stimulus later in a simulation in order to compare simulation results to experimental data where multiple perturbations are applied in series. To add time delays, change the parameter in the reaction diagram to have the following form,

$$
p=p_{\text {new }} \cdot\left(t>t_{1}\right)
$$

where $p$ is the parameter that you wish to change, $p_{\text {new }}$ is the new value for that parameter, and $t_{1}$ is the time at which the parameter will change. Then you can define $p_{\text {new }}$ and $t_{1}$ at the beginning of the simulation as described above.

On the "Solver" tab of the edit simulation window, you can specify the time period over which the simulation will be performed, as well as other properties such as the maximum time step and how many samples will be recorded. Since the experimental data which are used for our example were collected for 1,200 seconds at 10 seconds intervals, we set the ending time in the model to 1,200 and set the output interval to 10 seconds. Finally, with the fully defined simulation selected, the simulation can be run by pushing the "Run and Save Simulation" button. This will submit the simulation to be solved remotely on the Virtual Cell servers. When the model is solved, the "Running Status" column will read "completed". To view the results, push the "Simulation Results" button. This will bring 
up a window where you can view the simulation results for each of the different species $^{4}$. Also, the simulation values can be seen in table format by clicking the "Show Data" button in the bottom right corner, which can be useful for extracting data for further analysis or plotting. Figure 3.2 shows the results of the example model with addition of $1 \mu \mathrm{M}$ ISO at 200 seconds and the addition of the direct AC agonist forskolin (FSK, $50 \mu \mathrm{M}$ ) plus PDE inhibitor (IBMX, $100 \mu \mathrm{M}$ ) at 600 seconds.

\subsection{Collection of FRET Data for Model Integration}

Computational models of signaling dynamics often use FRET biosensors because of their ability to collect real-time signaling dynamics. Here we explain how to collect and analyze data obtained from FRET biosensors such that they can be used to evaluate and inform computational models. Various methods can be applied to measure FRET from the changes in donor and acceptor emission. FRET signal can be detected when an excited donor fluorophore transfers energy to an acceptor fluorophore in close proximity $(<10 \mathrm{~nm})$ [64]. A standard way to measure FRET is by quantifying the acceptor emission upon donor excitation (acceptor-sensitized emission). This method is usually corrected for spectral bleed-through caused both by leakage of the donor and acceptor emission into the FRET channel and by acceptor photobleaching. This method is also referred to as the 3-image FRET technique since it requires the measurement of 3 different intensities: acceptor emission, emission from the donor into the acceptor channel (due to FRET), and direct excitation of the acceptor. Our example uses two FRET biosensors, ICUE [65] and AKAR [66], which both utilize the Cyan and Yellow Fluorescence Proteins, CFP and YFP, a common and well established FRET

\footnotetext{
${ }^{4}$ The fluxes of each of the reactions can also be seen from this window, which can help to determine the source of errors if the simulation is not working as it should.
} 
A)

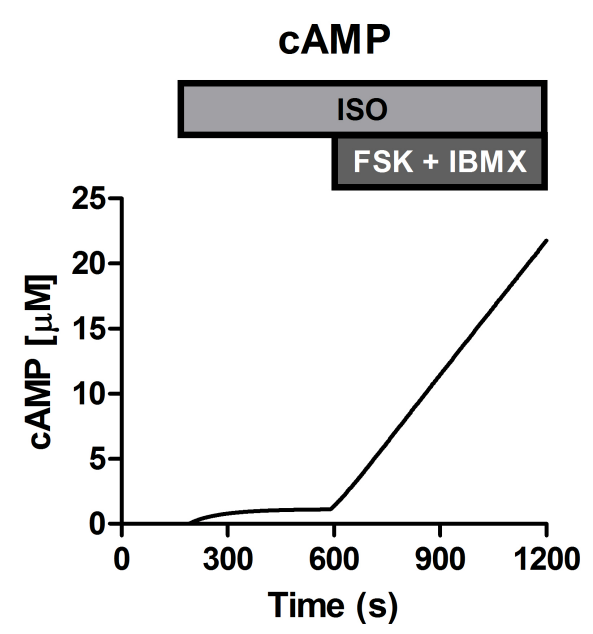

C)

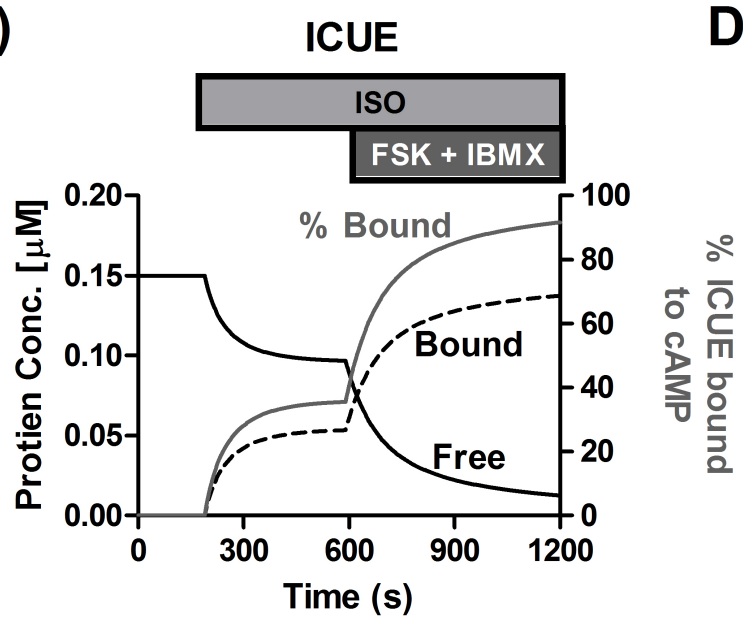

B)

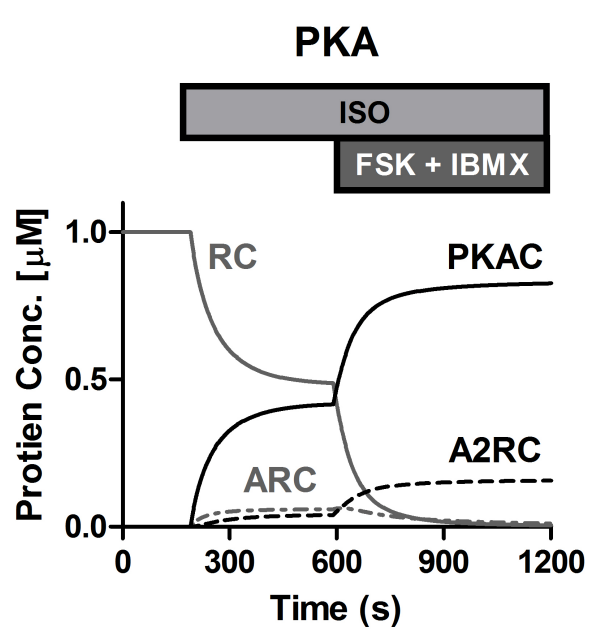

D)

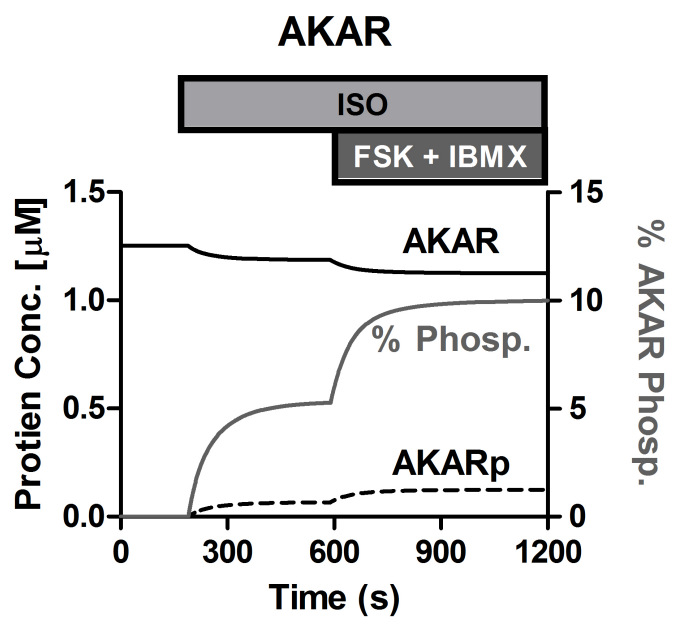

Figure 3.2: Initial Simulation Results: Simulation results with $1 \mu \mathrm{M}$ ISO applied at 200 seconds then $50 \mu \mathrm{M}$ FSK and $100 \mu \mathrm{M}$ IBMX applied at 600 seconds. A) cAMP concentration over time where the concentration grows unbounded in the model because cAMP generation becomes faster than the inhibited cAMP degradation. B) The four activation states of PKA. C) [Left axis] Concentrations of ICUE that are bound to cAMP (bound), and ICUE that does not have cAMP bound to it (free). [Right axis] The percent of total ICUE that has cAMP bound. D) [Left axis] Concentration of AKAR and AKARp. [Right axis] Percent of total AKAR that is phosphorylated. 
pair [74]. Since both ICUE and AKAR are unimolecular reporters, with fixed stoichiometry between donor and acceptor, the changes in donor emission ratio directly correlate to changes in FRET signal. The main purpose of this part of the chapter is to describe how to obtain data from FRET experiment which would be used for validation of the model predictions.

\section{FRET Experimental Data Collection and Normalization}

The generation of FRET experimental data for use in models consists of two interdependent parts: (A) collection of experimental data using FRET-based biosensors and (B) normalization of collected experimental data for model validation and integration. One can also use the model to predict the biological response of the signaling pathway to new perturbations and experimentally test these predictions to gain new insight into the modeling network behavior.

\section{Image Acquisition}

It is important to collect controls for minimal and maximal FRET signal to be able to normalize the changes in the FRET ratio and to determine fractional activation. Fractional activation is the fraction of the total biosensor that is activated (where 1 means the biosensor is fully activated and 0 means that biosensor is inactive). This is necessary because changes in the FRET ratio are a relative measure of changes in biological responses and normalization allows the absolute quantification of the biosensor response that is necessary for model validation and fitting. The minimal biosensor signal $\left(R_{\min }\right)$ is defined as FRET signal measured under conditions where basal enzyme activity or basic protein formation is inhibited by the appropriate inhibitors. In practice, determination of the minimal FRET signal $\left(R_{\min }\right)$ depends on the amount of basal activity or concentration of the protein of in- 
terest in the imaged cells. For example, protein kinase C (PKC) has a significant amount of basal activity, thus to measure the minimal FRET signal PKC inhibitors must be used [75]. In cardiac cells, we determined that cAMP levels are low in the resting state and that PKA has minimal basal activity, thus it is assumed that unstimulated cells exhibit the minimal biosensor signal without inhibition of baseline activity. To establish this baseline FRET for each AKAR or ICUE experiment, we usually collect data for the first $2-5$ minutes without addition of any drug. In contrast, the maximal biosensor signal $\left(R_{\max }\right)$ is determined by stimulating the signaling pathway in such a way that the FRET biosensor is maximally activated. This is usually done by applying a strong stimulator and inhibition of negative regulators of the signal response. To establish a maximal biosensor signal for cAMP or PKA, we add a mixture of $50 \mu \mathrm{M}$ FSK and $100 \mu \mathrm{M}$ IBMX $2-10$ minutes before the end of each experiment.

To visualize the reporters, we use the wide-field Olympus motorized inverted microscope IX81 equipped with an Olympus UPlanSApo 10X/1.3 numerical aperture (NA) objective. The Lambda DG4 excitation filter changer with Xenon arc lamp can be used as an illumination system designed for rapid wavelength change. Since the microscope for 3-color FRET must be capable of collecting donor fluorescence, acceptor fluorescence, and FRET emission, the Lambda 10-3 emission filter wheel changer with a Smart-Shutter is applied for automatic and fast switch between emission filters (for CFP and YFP). To filter excitation and emission wavelengths, we use an ECFP/YFP-ET filter set. The set contains beam splitter (CFP/YFP) and the following filter sets: for the YFP cube: excitation filter $500 / 20 \mathrm{~nm}$ and emission filter 535/30 nm; for the CFP cube: excitation filter 430/24 nm and emission filter 470/24 nm and for the FRET cube (CFP/YFP): excitation filter 430/24 $\mathrm{nm}$ and emission filter $535 / 30 \mathrm{~nm}$. To record the fluorescent images, we recommend using high-sensitivity cameras, as they will minimize the duration of excitation required to obtain 
the images, thereby reducing photobleaching of the sample and photodamage to the cell. We use a 12-bit Hamamatsu digital C9300-21 digital camera. Automated acquisition of images can be achieved using a variety of software packages, such as Metamorph or IP-LAB ${ }^{5}$.

\footnotetext{
${ }^{5}$ It is critical that the same settings and exposure times are maintained for each filter set during image acquisition of all samples.
} 


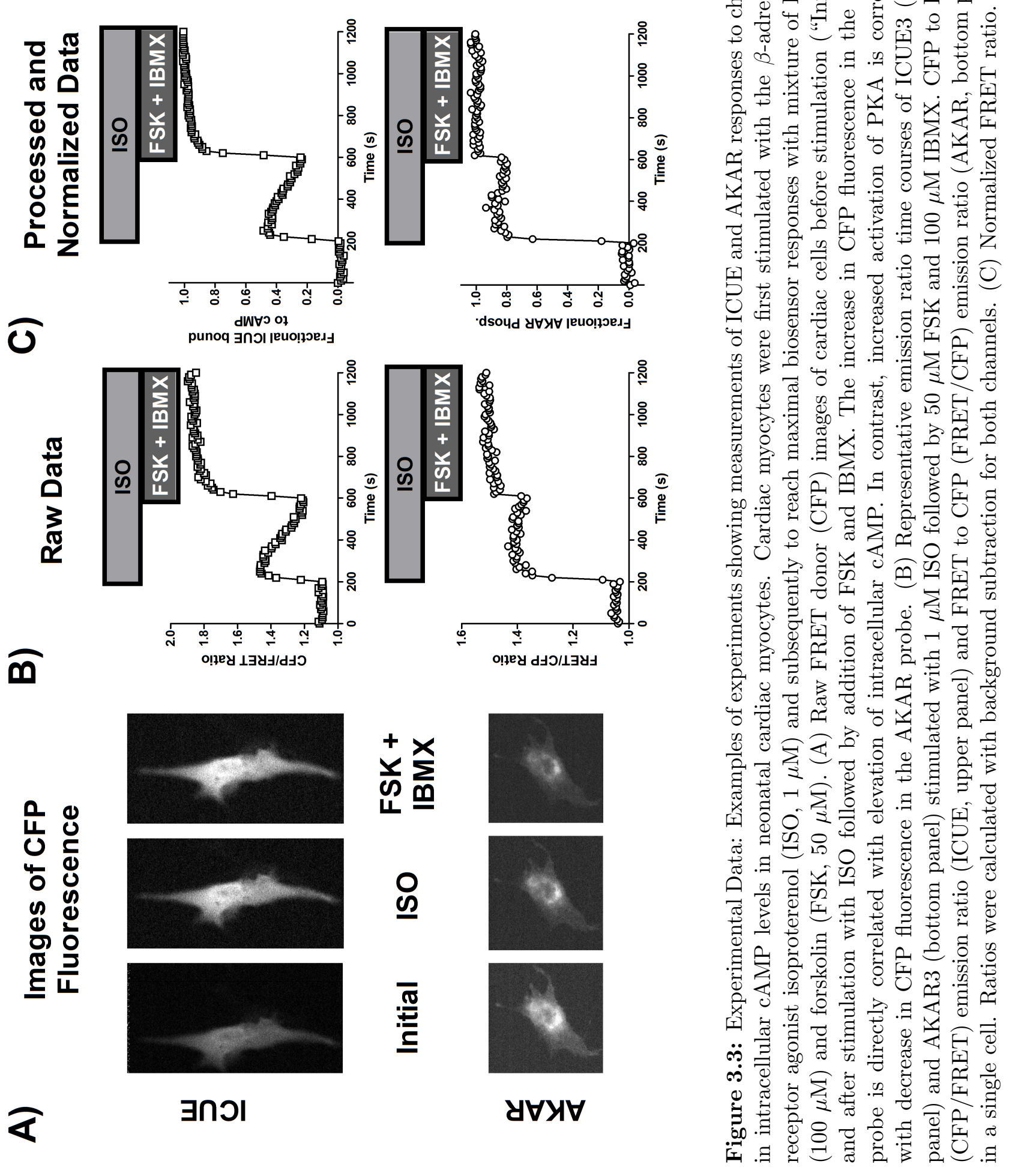




\section{Image Analysis}

To extract data obtained from an imaging experiment, open the recorded experiment and load the raw images using Image J software. Figure 3.3A shows an example of CFP images for both ICUE and AKAR in cardiac myocytes. Select the cells with similar intensity levels of CFP and YFP and draw ROIs both on the cell and on the background $(B k g r)$. The Image J software will extract the mean intensity values of $I_{A}, I_{D}$, and $I_{F}$ from the ROIs drawn. Save the experiment data as an .xls or .txt file.

To correct for background fluorescence, subtract the mean emission intensity of the background ROI from the corresponding mean emission intensity of the cell ROI for appropriate channels. Emission ratios are calculated from the collected data using the following formula for each time point:

$$
\begin{aligned}
& \text { Emission Ratio }(A K A R)=\frac{I_{F}-I_{F-B k g r}}{I_{D}-I_{D-B k g r}} \\
& \text { Emission Ratio }(I C U E)=\frac{I_{D}-I_{D-B k g r}}{I_{F}-I_{F-B k g r}}
\end{aligned}
$$

These data are plotted as changes in the corrected CFP/FRET and FRET/CFP emission ratios for ICUE and AKAR, respectively (Figure 3.3B). Spectral bleed-through correction in FRET biosensors can be done using the method described by Perisamay et al. [76]. For unimolecular FRET-based biosensors, such as ICUE or AKAR, the bleed-through correction can be omitted because of the fixed stoichiometry between the FRET pairs. In this case, the FRET data is expressed as direct fluorescence emission ratios from donor and FRET channels only with background $(B k g r)$ correction as indicated in step 2 (Figure 3.3B). Photobleaching can cause a progressive decrease in fluorescence and signal strength during image collection, which may interfere with FRET signal quantification. In addition, YFP bleaching results in an artificial CFP increase. Thus, illumination intensities and duration of cell 
illumination (exposure or acquisition time) should be kept as low as possible for minimizing photobleaching and phototoxicity. To correct for photobleaching, multiply the FRET channel intensity $\left(I_{F}\right)$ by the ratio of the acceptor intensity $\left(I_{A}\right)$ measured on direct acceptor excitation at the beginning of the experiment $\left(I_{A 0}\right)$ to the intensity at the corresponding time point during the experiment $\left(I_{A t}\right)$ [77]. Finally, calculate the average emission ratio of the minimal $\left(R_{\min }\right)$ and maximal biosensor signal $\left(R_{\max }\right)$ and normalize the raw emission ratio $(E M)$ with the following formula:

$$
\text { Normalized Emission Ratio }=\frac{E M-R_{\min }}{R_{\max }-R_{\min }}
$$

The normalized FRET data can be plotted on the scale from 0 to 1 (Figure 3.3C).

\subsection{Integrating Experimental Data}

\section{Model Validation}

Now that the computational model is built and some data has been acquired, the model needs to be compared with experimental data obtained using biosensors to validate that it captures the properties of the real biological system. The inclusion of experimental controls for minimal and maximal FRET biosensor signals allows us to directly relate the FRET signals to the fraction of the total biosensors that are active. If the control conditions do not span the entire activity range (0-100\%) the interpretation of the experimental data may be affected. To compare the computational results to the experimental data, the biosensor response must be normalized in terms of fractional activation. Fractional activation is calculated using the 
following algebraic equations:

$$
\begin{gathered}
I C U E \text { Ratio }=\frac{I C U E_{\_} c A M P}{\left(I C U E+I C U E_{\_} c A M P\right)} \\
A K A R \text { Ratio }=\frac{A K A R p}{(A K A R+A K A R p)}
\end{gathered}
$$

To have Virtual Cell calculate these algebraic equations, new global parameters need to be defined. In the "Parameters and Functions" section, click the "Add New Global Parameter" button which creates a new global parameter. Rename this parameter (e.g. ICUE_ratio) and in the expression column enter in the algebraic functions as shown above. Now you will be able to see the fractional activation of each biosensor in simulation results. Figure 3.4 shows the comparison between the base model that was created using the best initial guesses for kinetic rate parameters. This shows that the model in its current state does not accurately recapitulate the experimental evidence. This discrepancy can be caused by inaccuracies or uncertainty in kinetic parameters and protein concentrations. Therefore some parameter values will be adjusted so that the model predictions better fit the experimental data.

\section{Fitting to Experimental Data}

Gaps and uncertainty in information about model parameters can create discrepancies between model results and experimental data. To try to better capture the experimental data, we will vary some of the model parameters to fit to the experimental data. When fitting data, one must choose which parameters to fit based on the strength of evidence that was used to define that parameter. The goal is to fit as few parameters as possible but still accurately fit the biological response. When fitting parameters to data that monitor different aspects of a signaling network, such as ICUE monitoring cAMP concentration and 


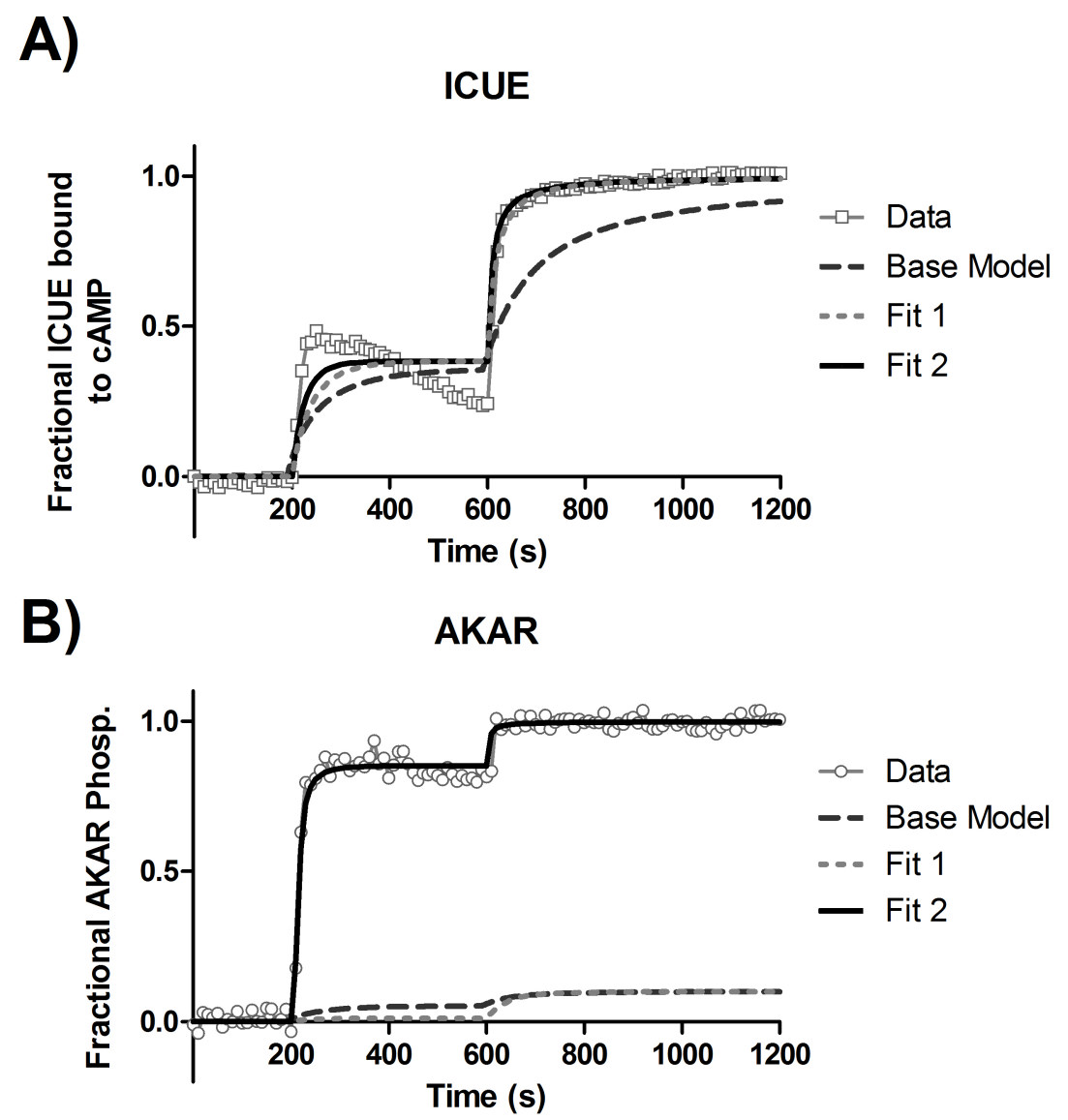

Figure 3.4: Data Normalization and Fitting: A) Normalized ICUE FRET data (squares) is compared to the ICUE ratios for computational models in different stages of the fitting process. B) Normalized AKAR FRET data (circles) is compared to AKAR ratios for computational models in different stages of the fitting process. Base model (Black dash) is the simulation results from the computational model that uses kinetic parameters from the literature. Fit 1 (gray dotted) is the simulation results after fitting kinetic parameters involved in cAMP production. Fit 2 (black solid) is the simulation results after fitting kinetic parameters from Fit 1 that are involved in PKA activation. 
AKAR reporting PKA activity, it can be more effective to fit different modules of the model separately. In this model, parameters involved in cAMP generation will be fitted first using the ICUE data (noted in Table 3.1 with $\dagger$ ). Then, when a good fit of cAMP production is achieved, parameters involving PKA activation will be fit against both ICUE and AKAR data. To use Virtual Cell to fit parameters, use the following steps:

To start, fitting is done in the "Parameter Estimation" tab of the Application that was defined earlier for your model. The first task is to define the parameters that will be varied to fit the experimental data. In the "Parameters" tab, click the add parameter button (green plus) and a list of all the model parameters will be shown. Select the parameters that will be varied and select "ok," which will put those parameters into the parameter tab. For each of the parameters that were selected, lower and upper bounds must be defined. One rule of thumb for the bounds is to allow one order of magnitude in both directions. This allows a fair amount of parameter variation but hopefully keeps parameters within a biologically relevant range.

Next, we will incorporate experimental data. To bring the experimental data into Virtual Cell, it must be saved in a comma separated values (.csv) format (which can be done using "save as" in Excel). The data and associated time values need to be put into columns with labels in the first row of the column. In the "Experimental Data Import" tab, click the "Import from CSV file" button and select the file where your data is saved. The graph should show the data from the file you just imported. The normalized data for this example can be extracted from the public model "ecg5pc: Greenwald MIMB 2012 - Fit 2". The data can be found in the "Experimental Data Import" tab of the Parameter Estimation section of the application. The data can be copied by selecting the "show data" button (blue grid), selecting all the data and copying it to a .csv file. When fitting to the ICUE data alone, do not import the AKAR data because Virtual Cell will try to fit the model to all data 
imported into that model.

In the "Experimental Data Mapping" tab, you need to relate the experimental data to Virtual Cell variables. When using biosensor data it will be necessary to use global variables to define fractional activity as explained earlier. For each data set in the file you imported, there will be a row that will be labeled with the title given in the data file. Select each row and push the "Map Experimental Data..." button. For that experimental data set, choose the appropriate global variable or species concentration to which it relates. For the normalized ICUE data, select the global parameter, as defined earlier, ICUE_ratio.

Finally, the fitting optimization can be done by selecting the "Run Task" tab. There are many methods to find the optimal parameters. We use the Levenberg-Marquadt method because it is an efficient and deterministic solution method. Once a solution method is selected, press the "Solve by Copasi" button and Virtual Cell will run the fitting algorithm. Once a solution is found, the fitted values for the parameters will be shown on the right. To inspect how well the fit agrees with the experimental data, you can push the "Plot" button. Also, the "save solution as new simulation" button is useful to record the results of the fit as well as provide the ability to examine this fit later.

The fit of the cAMP production, called Fit 1, is shown in Figure 3.4 and the model now has fairly good agreement with experimental ICUE FRET data. This model is not able to explain the decrease in ICUE response following ISO stimulation because the model did not include negative feedback loops in the $\beta$-adrenergic receptor pathway, including receptor desensitization and increased PDE activation by PKA-mediated phosphorylation. Such discrepancies between models and data can help identify what parts of a signaling network are necessary to capture a response. At this point, it may be useful to save a different copy of your model (File $>$ Save As...). After fitting the model to the ICUE data, we saved our model as the public model "ecg5pc: Greenwald MIMB 2012 - Fit 1". In 
this new copy of the model, the parameters that were fit in the previous iteration (for this model, cAMP generation) can be put into the model directly so that other components can be fit to the model data. With the "Fit 1" version of the model, we can now fit the parameters relating to PKA activity to the normalized AKAR data. The fit was done by again following steps described above, except we use both the normalized ICUE and AKAR data. Parameters chosen were both involved in PKA activation and catalytic activity as well as phosphatase activity, because the amount of AKAR phosphorylation is determined by the balance of PKA and phosphatase activity (parameters chosen indicated in Tables 3.1 and 3.2 by $\ddagger$ ). As it is shown in Figure 3.4, Fit 2 now has good agreement with both ICUE and AKAR data. This model is available as the public model "ecg5pc: Greenwald MIMB 2012 - Fit 2". To validate that the fitted model accurately represents the biological response, the model needs to be compared to an independent set of experimental data, distinct from the data used to fit the model.

Congratulations! Now you have successfully built a computational model, normalized experimental data to compare to model results and fit the model to the experimental data. Now we will provide some examples of how models can be used to test hypotheses.

\subsection{Interpreting Biosensor Data Using a Model}

The purpose of building models is to understand or evaluate hypotheses as well as create new testable hypotheses. One application of our example model is to identify the rate limiting step during PKA activation. This question was addressed by Saucerman et al. using the AKAR biosensor and a more complex model, finding that PKA activation kinetics are rate-limited by cAMP generation (see the Public Model "jsaucer: AKARmyocyte") [78]. Additionally, they were able to use this model with Virtual Cell's spatial modeling capabilities 
(not discussed here) to examine molecular mechanisms underlying subcellular PKA phosphorylation gradients. Violin et al. also used computational modeling to interpret experimental FRET results involving cAMP signaling [79]. Using ICUE, they examined how the duration of the cAMP signal is regulated in $\beta$-adrenergic signaling. They were able to validate their interpretation of experimental data by building a model that incorporated receptor desensitization through both $\beta$-arrestin and G-protein coupled receptor kinases as well as PKA-dependent PDE activation. Both of these examples exhibit the use of computational models to quantitatively validate hypotheses generated by experimental data.

Models can be used to generate hypotheses by simulating the effects of changing kinetic parameters or protein concentrations. To showcase some examples of model perturbations, here we test how biosensors may introduce three artifacts (biosensor saturation, buffering and kinetics) that affect biological interpretation of biosensor data. The model simulations of these perturbations can be found in the public model "ecg5pc: Greenwald MIMB 2012 - Perturb". One issue that can arise is that a strong biological response to a stimulus may saturate the biosensor. This can cause misinterpretation of biosensor data because the biological response may be greater than the biosensor is able to measure. We have used the model to simulate cases where ICUE responses can become saturated and no longer accurately reflect cAMP concentration. Figure 3.5A shows the ICUE response to different concentrations of the adenylyl cyclase agonist forskolin. As cAMP accumulates in cell, the ICUE response becomes saturated and unable to accurately report the cAMP concentration.

Biosensors can also have a direct impact on the biological system. For example, ICUE binds cAMP and thus can reduce the concentration of free cAMP. When a signaling molecule binds to a protein and removes it from solution, it is called buffering because it can maintain the concentration just like $\mathrm{pH}$ buffers can prevent the $\mathrm{pH}$ from changing. We used the model to show how changes in the ICUE concentration can affect the cAMP concentration in the 
cell (Figure 3.5B). As the concentration of ICUE increases, cAMP concentration equilibrates more slowly because the cAMP is first being bound to ICUE and then contributing to the cytosolic concentration. This shows that using cells with low biosensor expression minimizes the disturbance of the natural signaling kinetics.

Finally, it is important to understand the effect of biosensor kinetics on the measured pathway dynamics. If a biosensor has slow activation kinetics it can falsely represent the actual kinetics that are being monitored. Lai Hock Tay et al. observed this with the $\mathrm{Ca}^{2+}$ FRET biosensor TN-L15 and determined a method to quantitatively correct for the slow activation kinetics [80]. We modeled examples where ICUE binding kinetics would be slow by reducing both the binding and dissociation rates by an equal amount to only change the speed of binding and not the relative amount of cAMP bound. Figure 3.5C shows that as the ICUE kinetics get slower, the biosensor response becomes more dissimilar to the actual cAMP concentration. This is an important consideration in biosensor selection as well as design.

\subsection{Conclusions}

Computational modeling is a powerful tool for understanding and dissecting cell signaling that is often underutilized in biological studies. These models integrate biosensor data with biochemical data to be able to analyze the signaling network as a whole. Models can be used to both test hypotheses as well as generate new hypotheses. Models can also be useful for identifying and minimizing the impact of potential artifacts. It is the hope of the authors that this chapter allows experimental biologists to extract additional insights from their biosensor data using computational models. 


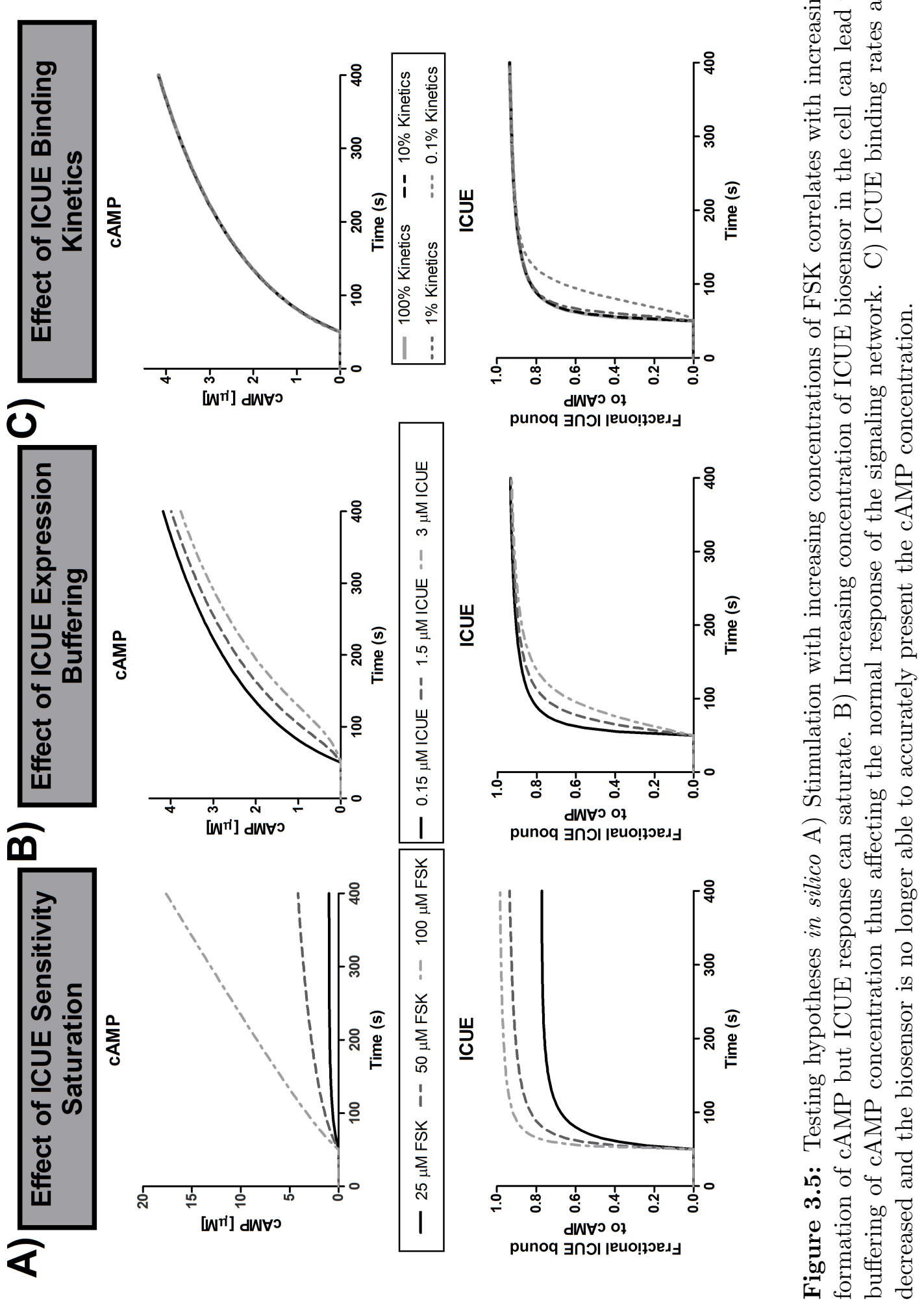




\section{Chapter 4}

\section{Scaffold state-switching amplifies,}

\section{accelerates and insulates PKC}

\section{signaling}

Reprinted from: E.C. Greenwald, J.M. Redden, K.L. Dodge-Kafka, J.J. Saucerman. "Scaffold StateSwitching Amplifies, Accelerates and Insulates PKC Signaling." Journal of Biological Chemistry Vol. 289 pp 2353-2360 (2014), with permission from the American Society for Biochemistry and Molecular Biology

Author contribution: E.C. Greenwald - Computational modeling and analysis, designed experiments, analyzed data, wrote the paper. - J.M. Redden - Performed experiments, designed experiments, analyzed data, assisted in writing the paper. K.L. Dodge-Kafka and J.J. Saucerman - Concieved the study, designed experiments, assisted in writing the paper. 


\subsection{Foreword}

In Chapter 2, we discussed the ability of scaffold proteins to accelerate, amplify and specify signal transduction to dynamically regulate numerous cellular processes. However, there is little theory available to mechanistically explain how signaling on protein scaffolds differs from solution biochemistry. Here, we propose the "scaffold state-switching" kinetic mechanism for enzymatic reactions on protein scaffolds to explain these phenomena, wherein the enzyme-substrate-scaffold complex undergoes stochastic state switching to reach an active state. In this chapter, we develop computational models of this novel scaffold state-switching mechanism and experimentally validate these models by studying the AKAP7 $\alpha$ tethered Protein Kinase C (PKC).

\subsection{Introduction}

Signaling enzymes transduce extracellular cues into cellular responses, often signaling via a wide cohort of effector proteins. The promiscuity of signaling enzymes has led to the evolution of scaffolding proteins and the advancement of the anchoring hypothesis. A growing body of evidence underlies this hypothesis, which states that the spatial sequestration of signaling enzymes with their substrate proteins is an important determinant of the efficacy and specificity of enzyme catalysis, most notably protein phosphorylation [81, 82]. Some scaffolds have been shown to accelerate or amplify signal transduction $[26,33]$, while others create specificity, allowing distinct context-dependent responses using the same promiscuous enzyme $[83,84]$. Although physiological and pathological roles have been identified for an increasing number of scaffolds, there is little mechanistic theory to explain how the colocalization provided by scaffolds modulates cell signaling. 
A-Kinase Anchoring Proteins, AKAPs, are a family of more than 50 functionally related yet structurally diverse proteins that have demonstrated many of these scaffolding phenomena [85]. While AKAPs were originally characterized by their ability to direct the actions of the cAMP-dependent protein kinase towards specific substrates, significant work has demonstrated that they function as more general scaffolds, integrating the actions of multiple enzymes [24]. For example, we have recently described the ability of AKAP7 $\alpha$ to localize the actions of PKC to a membrane domain [86]. Individual AKAPs have been shown to accelerate [33] or amplify [26] protein phosphorylation, yet it is unclear how these phenomena arise. They are hypothesized to be the result of enhanced enzyme-substrate interactions on a scaffold, but there is little quantitative evidence to explain how these macromolecular complexes actually influence enzyme catalysis.

Michaelis-Menten enzyme kinetics usually carry the assumption that both the enzyme and substrate are freely diffusing [87], but tethered enzymes and substrates contradict this assumption. In this chapter, we propose a novel mechanism for scaffold-tethered enzymatic reactions, the scaffold state-switching model. This model predicts that scaffold tethering of enzymes and substrates can lead to amplification and acceleration of signal transduction. These model predictions are validated experimentally by examining the kinetics of phosphorylation by $\mathrm{PKC}$ both on and off $\mathrm{AKAP} 7 \alpha$. We then investigated how scaffold tethering affected the sensitivity of PKC to different inhibitors. This analysis led to the surprising finding that AKAP7 $\alpha$ insulated PKC from ATP- and substrate-competitive inhibitors but not activation-competitive inhibitors. Further, our model demonstrated that insulation arose solely from scaffold tethering. The scaffold state-switching model provides a theoretical framework to study how and when acceleration, amplification and insulation emerge from scaffold localized reactions. 


\subsection{Methods}

\section{Expression constructs}

The following constructs were obtained from Addgene: PKC $\alpha$-Flag (plasmid \#10805), CKAR (plasmid \#10806), and MyrPalm-CKAR (plasmid \#14862). AKAP7 $\alpha$-CKAR was created by flanking AKAP7 $\alpha$ (Dr. John Scott, University of Washington) with HindIII restriction sites and subcloning it into the N-terminus of CKAR.

\section{Pharmacological Manipulations of PKC}

PKC activation was achieved using Phorbol-12,13-dibutyrate (PDBu, EMD Millipore \#524390) at a concentration of $250 \mathrm{nM}$. ATP competitive inhibition of PKC was achieved by preincubating transfected cells with 12-(2-Cyanoethyl)-6,7,12,13-tetrahydro-13-methyl-5-oxo5H-indolo(2,3-a)pyrrolo(3,4-c)-carbazole (Gö6976, EMD Millipore \#365250) at a concentration of $1 \mu \mathrm{M}$ for 5 minutes. The substrate competitive inhibitor PKC 20-28 (EMD Millipore \#476480) was incubated with cells for 30 minutes at $37^{\circ} \mathrm{C}$ at concentration of $16 \mu \mathrm{M}$. The activation inhibitor calphostin C (EMD Millipore \#208725) was pre-incubated with transfected cells at a concentration of $200 \mathrm{nM}$ for 30 minutes at $37^{\circ} \mathrm{C}$ followed by a 15 minute photoactivation via LED illumination.

\section{Fluorescence Resonance Energy Transfer (FRET)}

FRET measurements were executed as described previously [86]. Briefly, Vero cells were seeded at $50 \%$ confluency onto glass coverslips (Warner Instruments) and transfected with 500 ng plasmid DNA using the lipofectamine PLUS transfection system (Life Technologies). Cells were maintained at room temperature in imaging buffer $(\mathrm{pH} 7.3,172 \mathrm{mM} \mathrm{NaCl}, 2.4$ 
$\mathrm{mM} \mathrm{KCl}, 10 \mathrm{mM}$ HEPES, $4 \mathrm{mM} \mathrm{CaCl} 2,4 \mathrm{mM} \mathrm{MgCl}_{2}, 10 \mathrm{mM}$ glucose) for the duration of the imaging, which began following a 5 minute equilibration period on the microscope stage. All images were collected using a Zeiss Pascal confocal microscope and a 40x/1.2NA objective. Excitation of CFP was carried out using a $440 \mathrm{nM}$ laser (Toptica Photonics). A HQ535/50M and HQ480/40M emission filter with a 510DCLP dichroic were used (Chroma Technology). Channel intensities were quantified using ImageJ software. Individual traces were background corrected, standardized against their baseline values to put them on a scale of 1, and photobleach corrected against a linear fit of change in fluorescence for untreated cells. Data presented are composite traces from multiple cells and experiments as indicated within each figure.

\section{Confocal Imaging}

Representative images of MyrCKAR and AKAP7 $\alpha$-CKAR correspond to emissions collected in the CFP channel. Images were collected under identical conditions and settings, using the same laser $(440 \mathrm{nM})$ and filter configuration as the FRET experiments. Images were processed and arranged using ImageJ.

\section{Model Development}

A computational model was developed to describe PKC phosphorylation of CKAR both with and without the AKAP7 $\alpha$ scaffold, implemented in MATLAB (MathWorks). These models were based on mass action kinetics of either the standard enzyme mechanism or the scaffold state-switching mechanism. The model parameters were defined using literature values of biochemical rate constants. Parameters for phosphatase activity, basal activity and enzyme concentration were estimated by non-linear least-squares fitting to the CKAR 
experimental FRET measurements. Confidence intervals (95\%) on parameter estimates were calculated using the covariance matrix from the least squares fit. A detailed description of model equations and parameters is in Appendix A.

\section{Model Sensitivity Analysis}

Model sensitivity analysis was performed by randomly sampling kinetic parameter values from a fixed range of physiological values for the different rate constants as determined by a literature search (see Appendix A). The parameter space was sampled using a MATLAB built in Latin-Hypercube sampling algorithm to ensure complete coverage of the parameter space.

\section{Statistical analysis}

Unpaired t-tests were performed using GraphPad Prism 5.02 (GraphPad Software). P $<0.05$ was considered significant

\subsection{Results}

\subsubsection{Scaffold State-Switching Model Predicts Amplification and Acceleration of Reactions Occurring on Scaffolds}

The standard model of enzyme catalysis assumes that the enzyme and substrate freely diffuse in solution, reversibly associating to form an active intermediate state $([\overline{E S}])$ in which the enzyme catalyzes the conversion of the substrate to product $([E P])[87]$. Yet these assumptions are not valid for the tethered "solid-state" interactions that occur within an enzyme-substrate-scaffold complex. Thus we developed a new "scaffold state-switching" 
model to examine how tethering an enzyme and substrate to a scaffold protein affects enzyme catalysis. The key underlying assumption is that enzymatic reactions occurring on scaffolds exhibit stochastic switching of the enzyme-scaffold-substrate complex between active $(\overline{E-\kappa-S})$ and inactive $(E-\kappa-S)$ intermediate states (Figure 4.1). In this model we assume that the scaffold acts as a simple tether, although some scaffolds have been shown to directly regulate enzyme activity as well [88]. We defined a dimensionless number $\epsilon$ to compare the rate of enzyme-substrate interaction on the scaffold relative to that in solution. This "scaffold efficiency number" is defined as $\epsilon=k_{\epsilon} /\left(k_{f} \cdot[S]_{t o t}\right)$, where $\epsilon$ greater than 1 indicates faster enzyme-substrate interaction on the scaffold than in solution.

We next tested whether scaffold state-switching is sufficient to predict amplification and acceleration of signaling, as was observed experimentally for several AKAPs $[26,27,33,84]$. We modeled the activation of PKC and its phosphorylation of C-Kinase Activity Reporter, CKAR, in solution (Free, Figure 4.2A) and when tethered to a scaffold (Scaffold, Figure 4.2A). The "Scaffold" model assumes a scaffold state-switching mechanism, where active PKC and CKAR switch stochastically between active and inactive intermediate states. In both models, PKC is activated by the phorbol ester PDBu and CKAR is dephosphorylated by phosphatases. Rate constants were curated from the literature as detailed in Appendix A, with the scaffold efficiency number left as a free unknown parameter.

When the scaffold efficiency number is set to 1 , the active intermediate formation rate is the same on the scaffold and in solution, creating similar phosphorylation kinetics in solution and on the scaffold (Figure 4.2B). Increasing the scaffold efficiency number resulted in a concomitant increase in both the magnitude and rate of CKAR phosphorylation on the scaffold (Figure 4.2B, solid lines). These kinetic increases were quantified by changes in the fraction of phosphorylated CKAR and exponential time constant upon PDBu stimulation (Figure 4.2C). These model predictions show that when $\epsilon$ is greater than 1 , the enhanced 

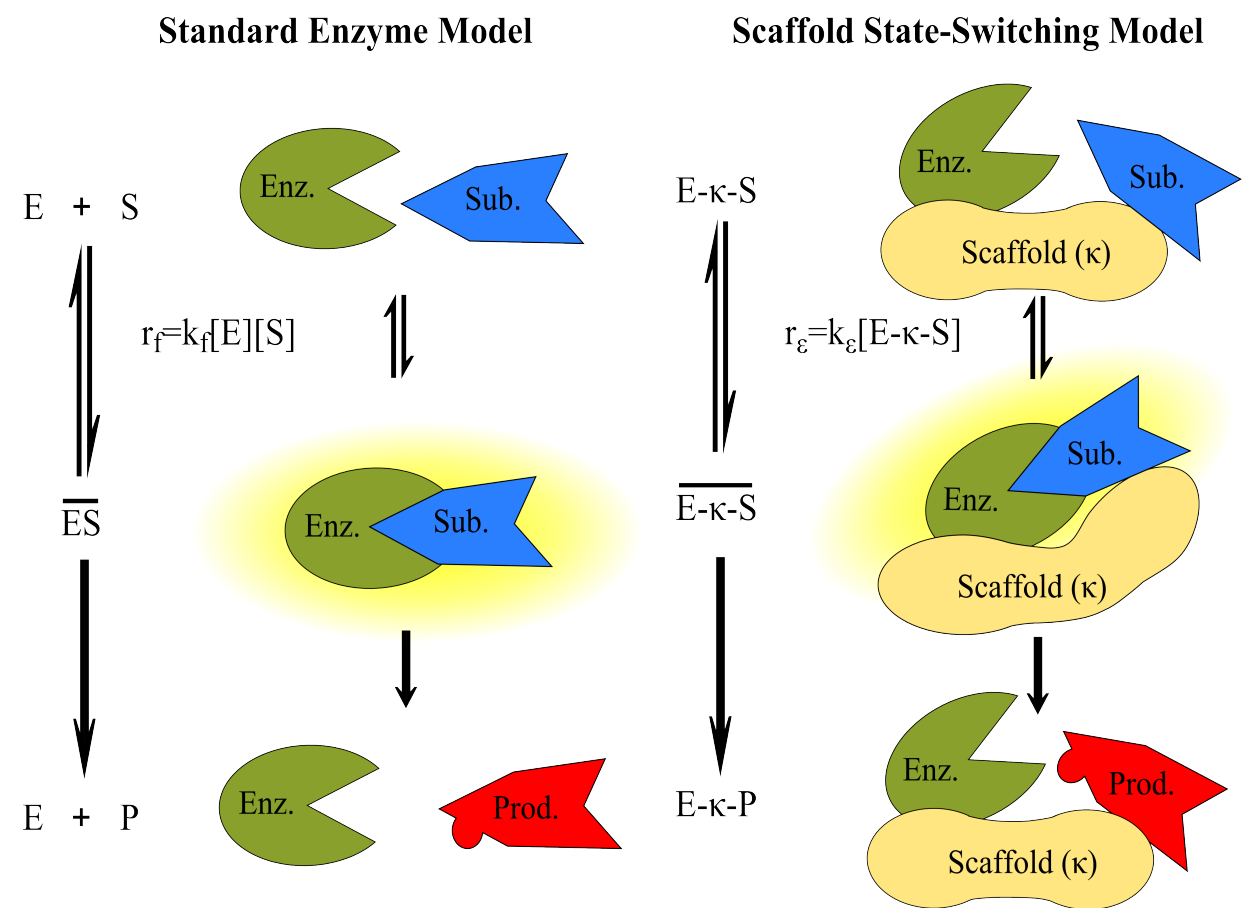

Figure 4.1: The scaffold state-switching model. Enzymes in solution follow standard enzyme kinetics (left), wherein an enzyme $(E)$ and substrate $(S)$ reversibly associate to form an active intermediate $(\overline{E S})$ and catalyze the formation of product $(P)$. The scaffold state-switching model (right), in contrast, describes anchored enzymatic reactions. In this model, both enzyme and substrate are bound to the scaffold $(\kappa)$, and the complex undergoes stochastic switching between inactive $(E-\kappa-S)$ and active $(\overline{E-\kappa-S})$ intermediate states through which the catalysis proceeds to form the product $(E-\kappa-P)$. Note that the enzyme substrate interaction rate on the scaffold $\left(r_{\epsilon}\right)$ is a first order reaction compared to the second order reaction in solution $\left(r_{f}\right)$. 
rate of active intermediate formation on a scaffold is sufficient to amplify and accelerate PKC signaling.

\subsubsection{AKAP7 $\alpha$ amplifies substrate phosphorylation by PKC as predicted by the Scaffold State-Switching model}

To experimentally validate our model predictions, we used the FRET biosensor CKAR, a live-cell kinetic reporter of intracellular PKC activity [79]. This biosensor undergoes FRET in its dephosphorylated state but loses this ability upon phosphorylation by PKC. We generated an anchored PKC activity reporter, AKAP7 $\alpha$-CKAR, by genetically fusing the PKC scaffold AKAP7 $\alpha$ to CKAR (Figure 4.3A). We have previously shown that AKAP7 $\alpha$ directly binds PKC with a $27 \mathrm{nM}$ affinity [86]. As AKAP7 $\alpha$ is membrane targeted in cells via Nterminal lipid modifications, we used myristolated CKAR, MyrCKAR, to represent the free substrate. This ensures comparative activation of PKC by PDBu for both biosensors. Validation of AKAP7 $\alpha$-CKAR was confirmed via western blot using anti-GFP and anti-AKAP7 antibodies (data not shown). Confocal images of the two biosensors demonstrate the constructs to be appropriately targeted to the plasma membrane with similar expression levels (Figure 4.3B). Anchored PKC-mediated phosphorylation, assessed by changes in AKAP7 $\alpha$ CKAR FRET ratio, was two-fold higher than the response of the free PKC reported by MyrCKAR $(7.4 \% \pm 0.7 \%$ AKAP7 $\alpha$-CKAR vs. $3.7 \% \pm 0.6 \%$ MyrCKAR) upon stimulation with PDBu (Figure 4.3C). We also observed a 1.8 fold acceleration of the phosphorylation rate of AKAP $7 \alpha$-CKAR, though this trend did not reach statistical significance. The amplified response of AKAP7 $\alpha$-CKAR was not simply due to overexpression of AKAP7 $\alpha$, because co-expression of AKAP7 $\alpha$ with MyrCKAR induced responses similar to MyrCKAR alone (Figure 4.4). We also examined whether the larger response of AKAP7 $\alpha$-CKAR was due to 
A

Free

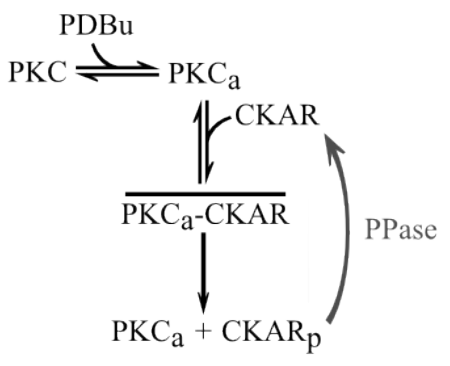

B

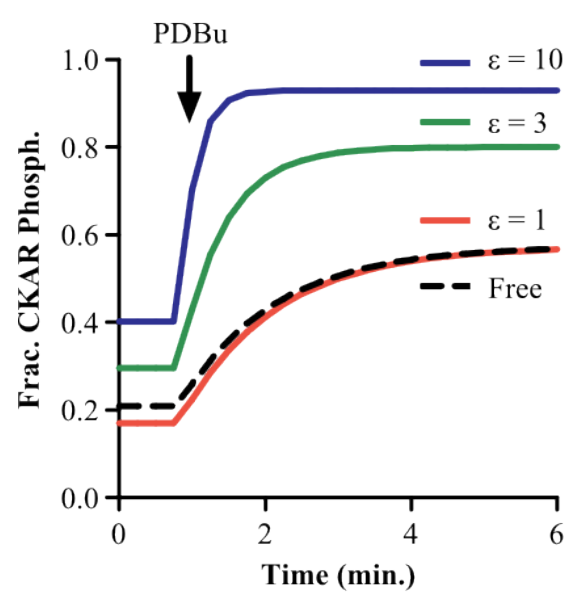

Scaffold

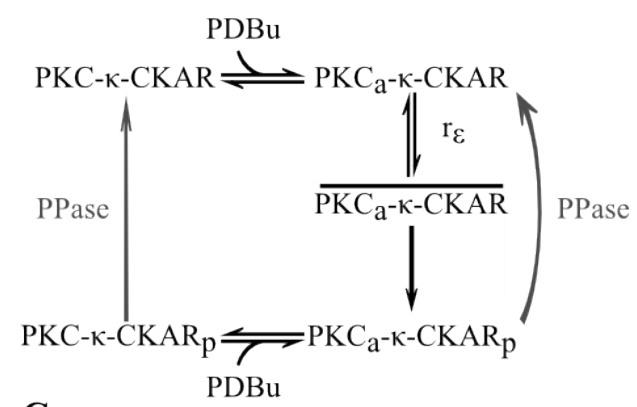

C

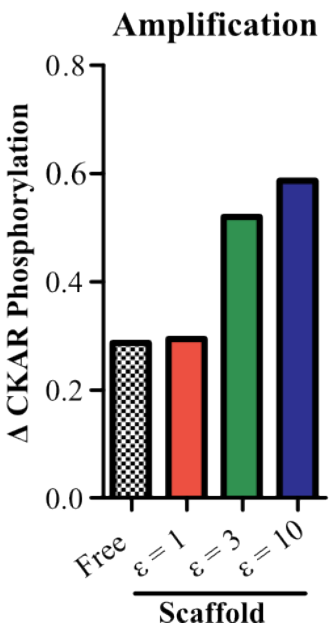

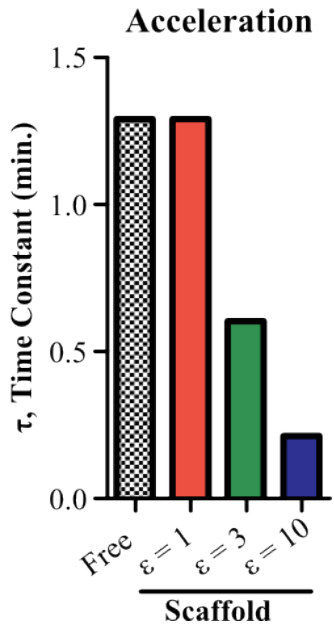

Figure 4.2: State switching model predicts acceleration and amplification of substrate phosphorylation. (A) Network diagram detailing assumptions of the computational models. In the free enzyme model (left), quiescent PKC is activated by $P D B u$. The active enzyme $\left(P K C_{a}\right)$ reversibly associates with its substrate, $C K A R$, to form the active intermediate $\left(\overline{P K C_{a}-C K A R}\right)$ and then carry out phosphorylation $\left(C K A R_{p}\right)$. However, in the scaffold state switching model (right), a pre-assembled complex containing PKC and CKAR exists on a scaffold $(P K C-\kappa-C K A R)$, causing $\mathrm{PDBu}$-bound $\mathrm{PKC}$ to switch stochastically between inactive $\left(P K C_{a}-\kappa-C K A R\right)$ and active $\left(\overline{P K C_{a}-\kappa-C K A R}\right)$ intermediate states. The efficiency of this state-switching mechanism is referred to as the scaffold efficiency number $(\epsilon)$. (B) The state-switching model predicts that increased scaffold efficiency results in a concomitant amplification and acceleration of CKAR phosphorylation. (C) Quantification of amplification and acceleration by the scaffold for increasing scaffold efficiency number. 
a larger dynamic range of this biosensor. Under conditions that maximize phosphorylation (combined PDBu and phosphatase inhibitor Calyculin A), AKAP7 $\alpha$-CKAR and MyrCKAR exhibited similar responses (Figure 4.5). Thus, we conclude that the observed increases in PKC-mediated phosphorylation reported by AKAP7 $\alpha$-CKAR are indeed due to scaffold efficiency, and not due to perturbation of the cellular environment.

We refined our model parameters by simultaneously fitting the free and scaffold models to the MyrCKAR and AKAP7 $\alpha$-CKAR data, respectively. All parameter values for free and scaffold models were constrained to be equal, with the exception of the rate constant for active intermediate formation on the scaffold $\left(k_{\epsilon}\right)$ and in solution $\left(k_{f}\right)$. During the fit, both models were scaled to the measured steady state PDBu response of MyrCKAR (for further explanation see Appendix A). The fitted model was in good agreement with the experimental data for both MyrCKAR and AKAP7 $\alpha$-CKAR (Figure 4.3C). By fitting the model to these data, we inferred a scaffold efficiency number of $\epsilon=5.70 \pm 0.37$ for PKC on AKAP7 $\alpha$. Similar to the experimental data, the model predicts that AKAP7 $\alpha$ amplifies PKC signaling in response to PDBu (Figure 4.3D, left). The simulations also show a $61 \%$ percent decrease in the time constant of CKAR phosphorylation on AKAP7 $\alpha$, consistent with the trend measured experimentally (Figure 4.3D, right). Collectively, these data and simulations indicate that the amplification and acceleration of PKC phosphorylation of substrates mediated by AKAP7 $\alpha$ are caused by a roughly 5 -fold increase in the rate of active intermediate formation. 
A

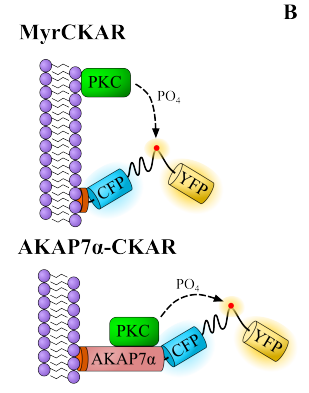

B

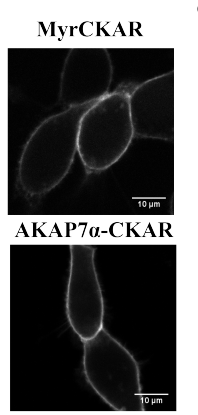

C

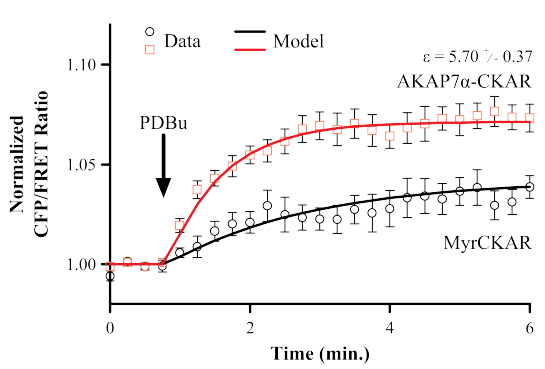

D

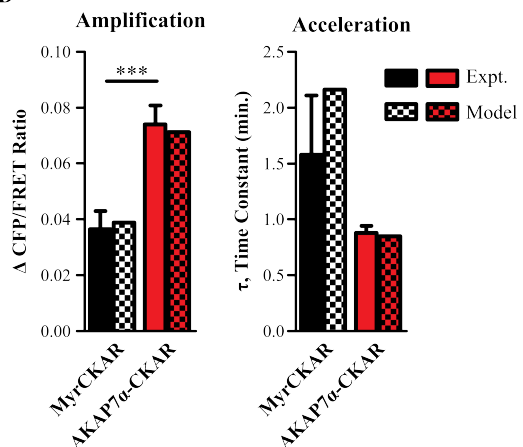

Figure 4.3: AKAP7 $\alpha$ accelerates and amplifies CKAR phosphorylation. (A) Schematic diagram of the FRET probes MyrCKAR (top) and AKAP7 $\alpha$-CKAR (bottom). MyrCKAR, characterized previously, contains an N-terminal Myr/Palm sequence sufficient to target it to the plasma membrane. AKAP7 $\alpha$-CKAR, generated by fusing AKAP7 $\alpha$ to the CKAR backbone, is also membrane targeted by virtue of a Myr/Palm domain contained within the N-terminus of the AKAP. (B) Confocal images of MyrCKAR and AKAP7 $\alpha$-CKAR obtained in the CFP channel (440 nm laser). (C) Cells expressing MyrCKAR (black circles) $(\mathrm{n}=11)$ or AKAP7 $\alpha$-CKAR (red squares) $(\mathrm{n}=9)$ were stimulated with $250 \mathrm{nM}$ PDBu exhibit increases in the FRET ratio. Both the free model (black line) and scaffold model (red line) were simultaneously fit to the MyrCKAR and AKAP7 $\alpha$-CKAR data, respectively, resulting in a scaffold efficiency number $(\epsilon)$ of $5.70 \pm 0.37$ (95\% confidence interval). (D) Quantification of experimental data (solid bars) and corresponding model fits (checkered bars) reveal amplification and a trend towards acceleration of AKAP7 $\alpha$-CKAR (red) vs. MyrCKAR (black). All error bars are standard error, ${ }^{* * *} \mathrm{p}<0.001$.

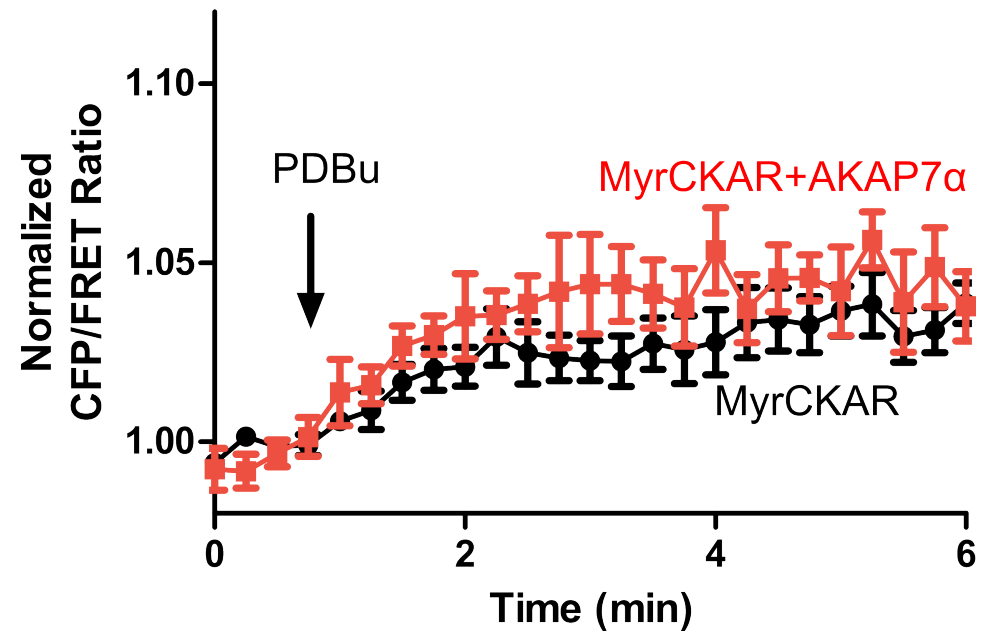

Figure 4.4: Co-expression of AKAP7 $\alpha$ does not affect responses of MyrCKAR. Cells expressing expressing MyrCKAR (black) were compared to cells co-expressing MyrCKAR and AKAP7 $\alpha$ (red). In response to $250 \mathrm{nM} \mathrm{PDBu}$, co-transfected cells had a $3.8 \% \pm 0.9 \%$ increase in CFP/FRET ratio $(n=6)$, compared with a $3.7 \% \pm 0.6 \%$ increase in CFP/FRET ratio in cells expressing MyrCKAR alone $(\mathrm{n}=11)$. 


\subsubsection{AKAP $7 \alpha$ insulates PKC from substrate- and ATP-competitive inhibitors but not activation-competitive inhibitors}

In addition to amplification and acceleration, scaffold tethering may impact other aspects of cell signaling such as the sensitivity to outside regulators. We sought to test if the scaffold state-switching model can predict whether scaffolds insulate PKC from certain classes of inhibitors, lowering their effective potency in live cells. We hypothesized that the scaffold would cause PKC to interact preferentially with tethered CKAR, insulating PKC from a substrate-competitive inhibitor in solution. Conversely, we hypothesized that activationand ATP-competitive inhibitors would be similarly potent for PKC with and without the scaffold. To evaluate this hypothesis in silico, we extended our model to incorporate these three different classes of inhibitors (Figure A.3). We simulated the effect of each inhibitor on the steady state response of $\mathrm{PKC}$ in the presence of $\mathrm{PDBu}$, both with and without scaffold (Figure 4.6). Results were normalized to the PDBu-stimulated increase in CKAR phosphorylation without inhibitor.

The activation-competitive PKC inhibitor was modeled by assuming that it competed with the binding of PDBu and endogenous lipid activators of PKC. The model predicted similar potency of activation-competitive inhibitor for both free PKC and scaffold-tethered PKC, consistent with our hypothesis (Figure 4.6A). Note that the rise of the normalized inhibitor response curve at low doses of calphostin $\mathrm{C}$ is due to a greater effect on basal PKC activity than PDBu-stimulated PKC activity.

The substrate-competitive PKC inhibitor was modeled by assuming that it reversibly associates with active PKC when PKC and the substrate are not in the active intermediate state. The substrate-competitive inhibitor was predicted to have a 3.7-fold lower potency for scaffold-tethered PKC compared to free PKC, consistent with our hypothesis above (Fig- 


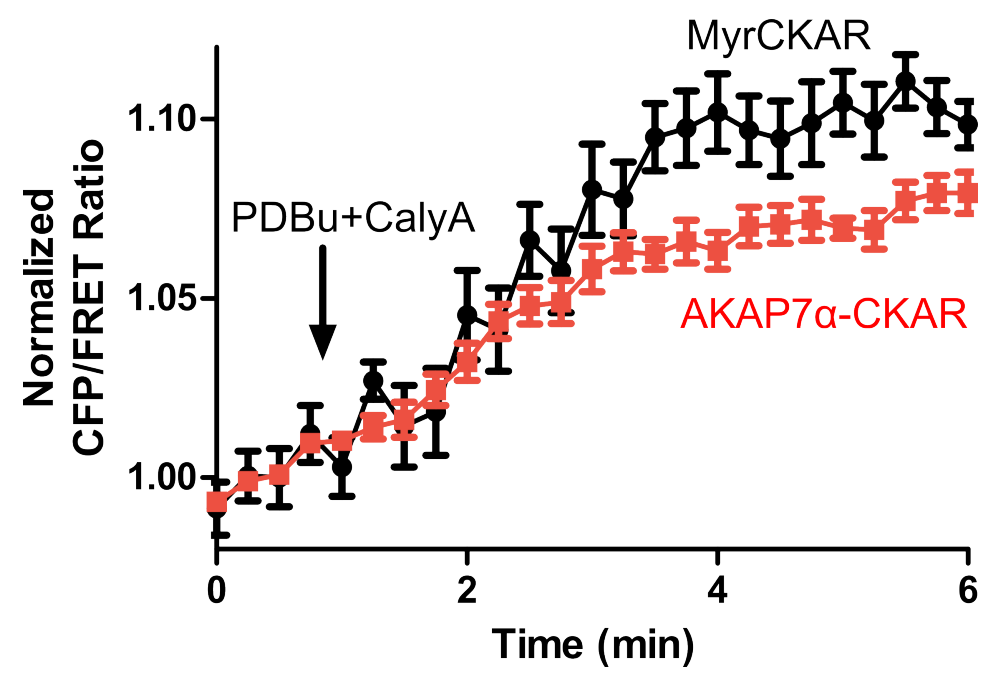

Figure 4.5: AKAP $7 \alpha$-CKAR does not have a larger dynamic range than MyrCKAR. In response to combined PKC activation $(250 \mathrm{nM} \mathrm{PDBu})$ and phosphatase inhibition (50 nM calyculin A), cells expressing AKAP7 $\alpha$-CKAR increased CFP/FRET ratio by $7.6 \% \pm 0.6 \%(\mathrm{n}=12)$ while cells expressing MyrCKAR increased CFP/FRET ratio by $9.8 \% \pm 0.7 \%(\mathrm{n}=12)$.

A
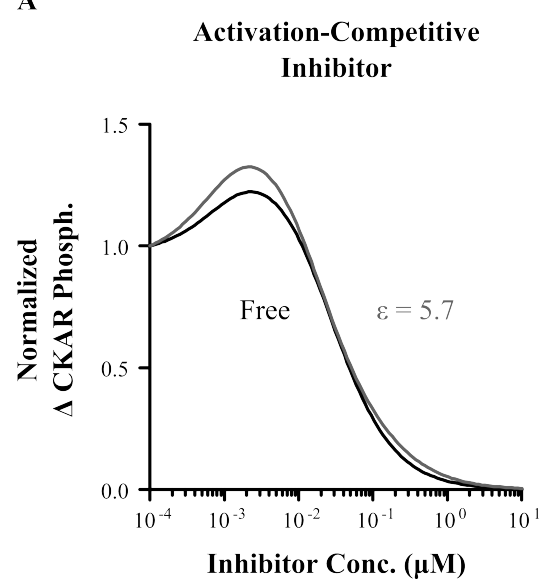

Substrate-Competitive Inhibitor

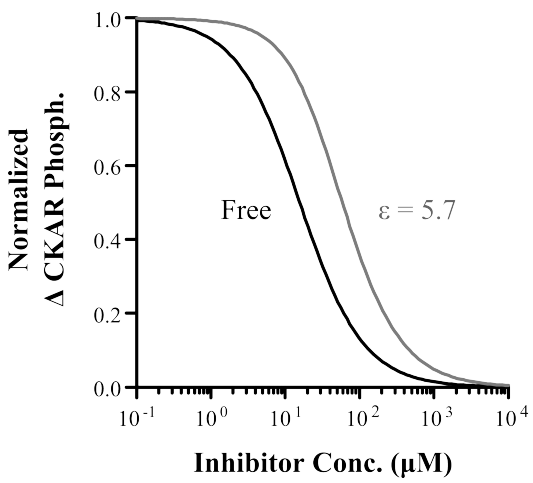

C
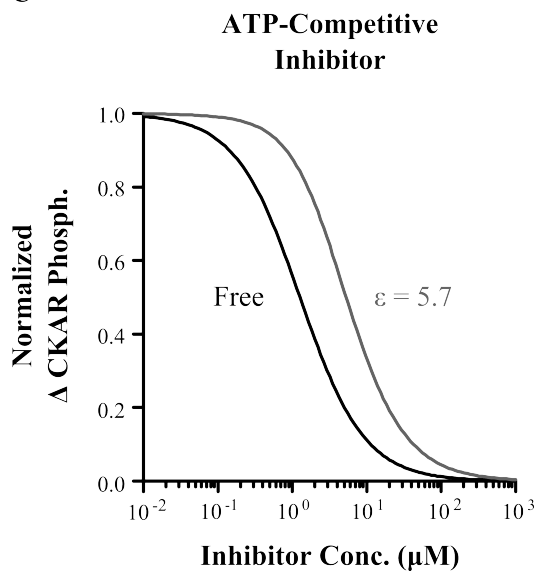

Figure 4.6: State-switching model predicts insulation of anchored PKC. Dose responses of PKC to three different classes of inhibitors were simulated, where the PKC activity was quantified by the change in CKAR phosphorylation upon stimulation with PDBu. All responses are normalized to the non-inhibited response to $\mathrm{PDBu}$. The kinetic parameters from the fitted models were used in the simulation $(\epsilon=5.7)$. (A) Activation-competitive inhibitors were predicted to have similar potency either with $(\epsilon=5.7)$ or without (Free) tethering to the scaffold. (B) Substrate-competitive inhibitors acting on anchored $\mathrm{PKC}$ were predicted to have reduced potency (increased $\left.I C_{50}\right)$ compared to free enzyme. (C) ATP-competitive inhibitors were also predicted to exhibit lower potency for anchored PKC vs. free PKC. 
ure 4.6B). To understand the mechanism underlying how the scaffold insulated the kinase from substrate-competitive inhibitors, we examined how the amount of enzyme-substrate active intermediate depends on the inhibitor concentration (Figure A.4). This analysis showed that because scaffold tethering increased the amount of active intermediate, more inhibitor was needed to obtain active intermediate levels (and downstream CKAR phosphorylation) similar to that without scaffold. In contrast, scaffold-induced insulation was not predicted for activation-competitive inhibitors because the amount of PKC that becomes activated is independent of the enzymatic reaction efficiency.

The ATP-competitive PKC inhibitor was modeled by assuming that it reduces PKC's effective catalytic rate constant by competing with ATP in a rapid equilibrium manner. In contrast to our original hypothesis, the model predicted that the ATP-competitive inhibitor would have a surprising 4.2-fold lower potency for scaffold-tethered PKC compared to free PKC (Figure 4.6C). To understand why the model predicted insulation for ATPcompetitive inhibitors, we examined the effect of changing the catalytic rate constant $\left(k_{\text {cat }}\right)$ on the magnitude of CKAR phosphorylation (Figure A.5). This analysis showed that by increasing the efficiency of phosphorylation, the scaffold pushed the system to a state where catalytic rate constant is less rate-limiting to phosphorylation. Thus, by increasing the rate of enzyme-substrate active intermediate formation, scaffolds may create a "catalytic reserve" that requires more inhibitor to obtain a similar amount of inhibition. This counter-intuitive insulation of ATP-competitive inhibitors suggests that insulation is a novel property of scaffold tethering. Thus, the scaffold state-switching model predicted that substrate tethering can insulate PKC from ATP- and substrate-competitive inhibitors but not an activationcompetitive inhibitor.

To test the model predictions of inhibitor insulation, we performed experiments comparing the sensitivity of MyrCKAR and AKAP7 $\alpha$-CKAR to calphostin C (an activation- 
competitive PKC inhibitor), Gö6976 (an ATP-competitive PKC inhibitor) and pseudosubstrate oligopeptide PKC 20-28 (a substrate-competitive PKC inhibitor). In agreement with the model predictions, pre-treatment with the activation inhibitor calphostin $\mathrm{C}$ significantly inhibited PKC substrate phosphorylation elicited by PDBu on both MyrCKAR (109.8\% inhibition) and AKAP7 $\alpha$-CKAR (71.5\% inhibition) (Figure 4.7A). Strikingly, we found that while the pseudosubstrate inhibitor 20-28 robustly suppressed MyrCKAR phosphorylation (72.0\% inhibition), PKC tethered to AKAP7 $\alpha$ was insulated from this substrate-competitive inhibitor (6.4\% inhibition). These experimental data indicate that AKAP7 $\alpha$ insulates PKC from substrate competitive inhibitors even more than predicted by the computational model (Figure 4.7B). Similarly, although the ATP-competitive inhibitor Gö6976 was a potent inhibitor of MyrCKAR phosphorylation (72.2\% inhibition), Gö6976 did not significantly decrease PKC activity on AKAP7 $\alpha$-CKAR (Figure 4.7C). This AKAP7 $\alpha$-induced insulation of PKC from Gö6976 qualitatively agreed with the lower potency of ATP-competitive inhibitors predicted by the model. Indeed, the experimentally measured insulation was even greater than in the model, perhaps due to underestimation of the scaffold efficiency number. Thus these experiments successfully validate model predictions of drug sensitivity for PKC tethered to AKAP7 $\alpha$ for 3 distinct classes of inhibitors. 
A

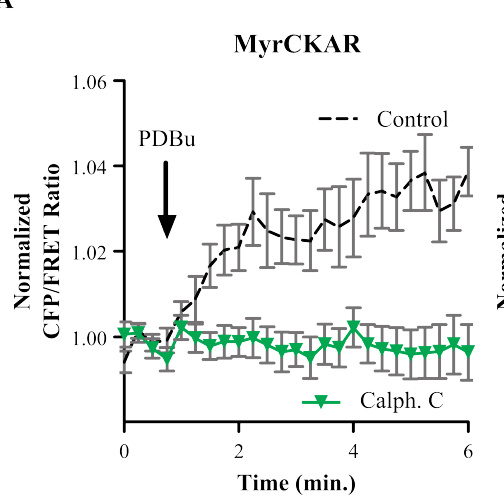

B

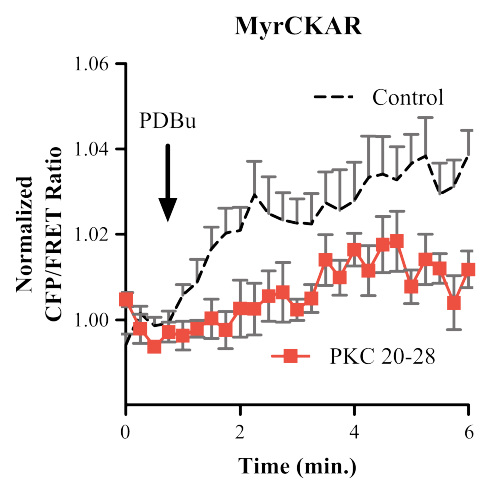

C

MyrCKAR

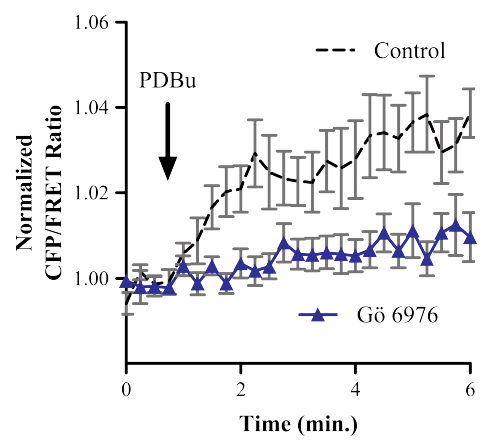

AKAP7 $\alpha$-CKAR

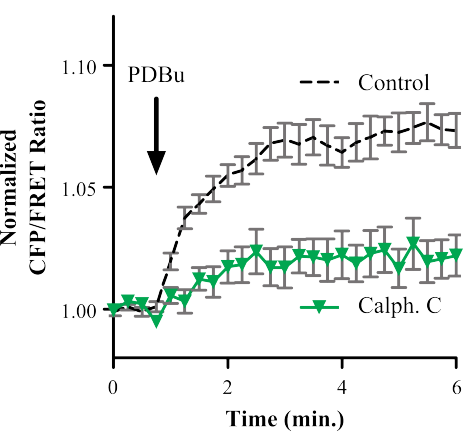

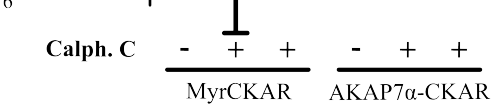

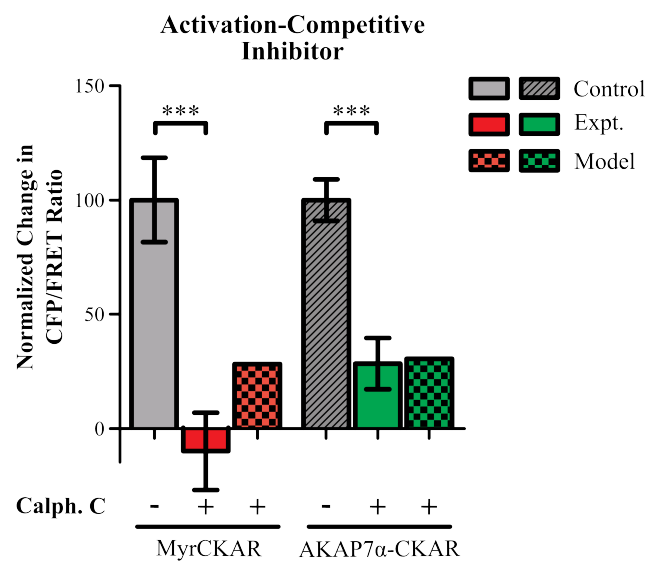

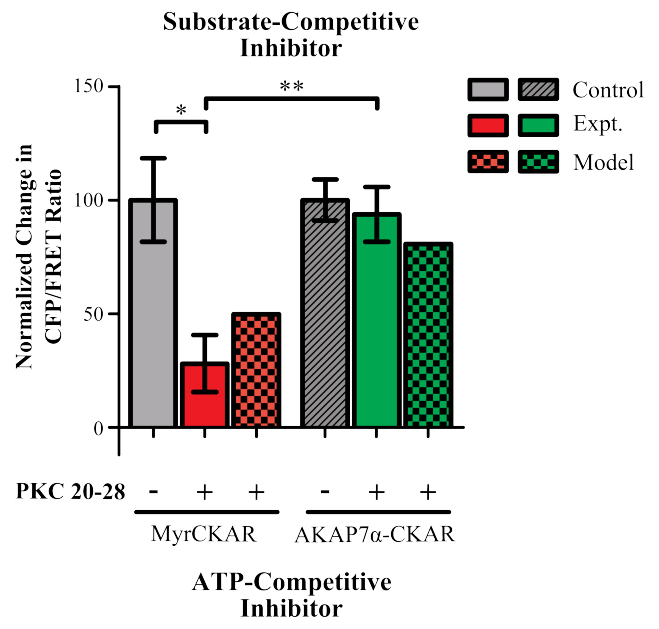
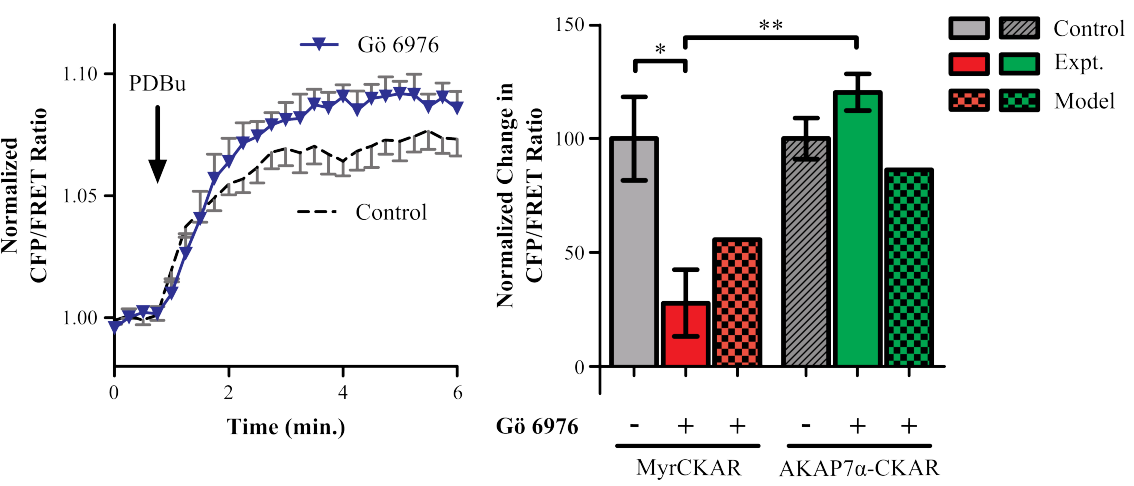
Figure 4.7: PKC tethered to AKAP7 $\alpha$ is insulated from certain classes of pharmacological inhibitors. (A) Cells expressing either MyrCKAR $(\mathrm{n}=17)$ or AKAP7 $\alpha$-CKAR $(\mathrm{n}=12)$ were pretreated with the activation-competitive PKC inhibitor calphostin C (200 nM). Calphostin C significantly inhibited the response of MyrCKAR to PDBu (solid red bar). Similarly, AKAP7 $\alpha$-CKAR was also significantly inhibited by calphostin $\mathrm{C}$ (solid green bar). The difference in the amount of inhibition between MyrCKAR and AKAP7 $\alpha$-CKAR was not significant $(\mathrm{p}=0.099)$. These measurements agree with predictions from the free enzyme model (checkered red bar) as well as in the AKAP7 $\alpha$ scaffold state switching model $(\epsilon=5.7$, checkered green bar). (B) Cells expressing either MyrCKAR $(\mathrm{n}=8)$ or AKAP7 $\alpha$-CKAR $(\mathrm{n}=8)$ were pre-treated with the substrate-competitive PKC inhibitor PKC 20-28 (16 $\mu \mathrm{M})$. PKC 20-28 significantly inhibited PDBu-dependent responses of MyrCKAR (solid red bar), yet no significant inhibition was observed for AKAP7 $\alpha$-CKAR (solid green bar). The inhibition of MyrCKAR was significantly greater than that of AKAP7 $\alpha$-CKAR, consistent with predictions from the free (checkered red bar) and the scaffold model $(\epsilon=5.7$, checkered green bar). (C) Cells expressing either MyrCKAR $(\mathrm{n}=9)$ or AKAP7 $\alpha$-CKAR $(\mathrm{n}=7)$ were pre-treated with the ATP-competitive PKC inhibitor Gö6976 (1 $\mu \mathrm{M})$. Gö6976 significantly inhibited the PDBu-evoked FRET response of MyrCKAR (solid red bar). In contrast, no significant inhibition was observed for AKAP7 $\alpha$-CKAR (solid green bar). MyrCKAR inhibition was significantly greater than AKAP $7 \alpha$-CKAR, in agreement with the model-predicted lower potency of Gö6976 for the scaffold (checkered green bar) compared to free PKC (checkered red bar). All error bars are standard error, ${ }^{*} \mathrm{p}<0.05,{ }^{* *} \mathrm{p}<0.01,{ }^{* * *} \mathrm{p}<0.001$.

\subsubsection{Extent of Acceleration, Amplification and Insulation Vary Depending on Enzyme Kinetics}

Here we have focused on how AKAP7 $\alpha$ tethering modulates PKC signaling. However, as numerous other enzyme-substrate-scaffold complexes exist within cells, we sought to determine if these same principles can be applied to other scaffold complexes as well. From the literature we identified biologically plausible ranges of kinetic rate parameters for select protein kinases (e.g. PKA, CaMKII) and phosphatases (e.g. PP1, PP2A). Parameters were randomly sampled within these ranges to obtain models for 2000 distinct hypothetical protein complexes. To allow fair comparison between complexes, the scaffold efficiency number was held constant at the level estimated for PKC-AKAP7 $\alpha(\epsilon=5.7)$. Each hypothetical protein complex was simulated to quantify the predicted acceleration, amplification and insulation. The dis- 
tribution and co-variation of these features were examined to identify the range of possible scaffold "phenotypes" (Figure 4.8). Overall, this analysis demonstrated that amplification, acceleration and insulation are expected to arise for many protein complexes, although their quantitative levels can vary considerably. Amplification and acceleration showed a negative correlation, meaning that there are some cases where a scaffold can greatly amplify the response of a kinase but the rate of the response will have very little acceleration, and vice versa (Figure 4.8A). Similarly, insulation and amplification were negatively correlated (Figure 4.8B). This strong negative correlation arose because kinases that fully phosphorylated their substrate without the scaffold could not exhibit further amplification when tethered to the scaffold. Yet these kinases that exhibited high activity without the scaffold had a larger catalytic reserve, leading to a larger insulation from inhibitor when tethered to the scaffold. Examples of specific responses can be seen in Figure A.6. We verified that the trends seen in this analysis are not strictly correlated with any single kinetic parameter but rather a result of the effect of the scaffold on the system as a whole (Figure A.7). Overall, this sensitivity analysis showed that the extent of acceleration, amplification and insulation by a scaffold can vary depending on the rate constants of the particular kinase and phosphatase.

\subsection{Discussion}

We propose scaffold state-switching as a novel mechanism shaping the kinetics of signaling on protein scaffolds. This model assumes that enzymes and substrates tethered to a scaffold switch stochastically between active and inactive intermediate states. Our computational models and subsequent experimental validation showed that the scaffold state-switching model accurately predicted the amplification and acceleration of PKC phosphorylation of a tethered substrate. Reconciling our computational and experimental data, we estimated 
A

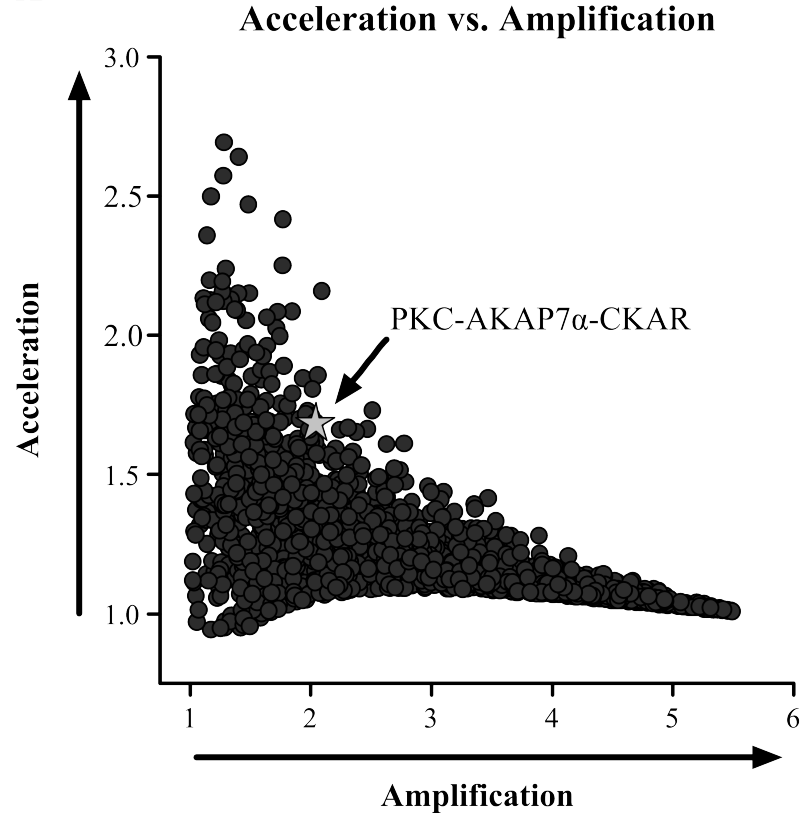

B

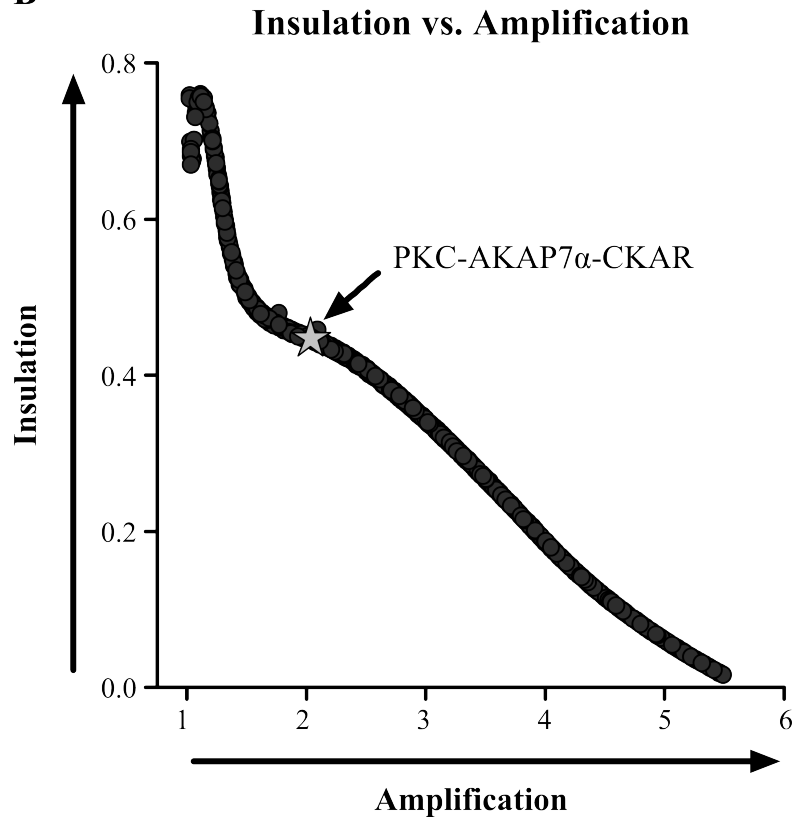

Figure 4.8: Amplification, acceleration and insulation vary depending on enzyme kinetics. Enzyme kinetic parameters were varied over a physiological range to produce models of 2000 hypothetical enzyme-substrate-scaffold complexes (black circles). For all simulations the scaffold efficiency number was held constant at $\epsilon=5.7$. The PKC-AKAP7 $\alpha$-CKAR model is indicated as a gray star. (A) Amplification is negatively correlated with acceleration. Amplification was quantified as the ratio of the steady state substrate phosphorylation $\left(S_{p}\right)$ for scaffold to solution biochemistry, $S_{p}(S c a f f o l d) / S_{p}($ Free $)$. Acceleration was quantified as the ratio of half maximal time, $t_{50}$, in solution to on the scaffold, $t_{50}($ Free $) / t_{50}($ Scaffold $)$. (B) Insulation and amplification are also negatively correlated. Insulation from substrate-competitive inhibitors was quantified as the log of the ratio of the $I C_{50}$ on scaffold to in solution, $\log 10\left(I C_{50}(S c a f\right.$ fold $) / I C_{50}($ Free $\left.)\right)$. 
that AKAP7 $\alpha$ tethering increased PKC's rate of association with its substrate by more than 5 -fold. We then extended our model to address how scaffolding proteins could affect the external influence of three classes of pharmacological kinase inhibitors. Both our computational simulations and experimental evidence demonstrated that AKAP7 $\alpha$ insulated bound PKC from substrate- and ATP-competitive inhibitors. However, no protection was offered against activation-competitive inhibitors. Finally, model sensitivity analysis indicated that scaffolds may amplify, accelerate and insulate a broad range of signaling pathways. Overall this work provides a new kinetic mechanism for scaffold-localized reactions and a theoretical underpinning with which to further understand how scaffold proteins shape cell signaling.

Acceleration and amplification by scaffold proteins has been shown for several different protein complexes $[26,27,33]$. Physiological and pathological roles of acceleration and amplification by scaffolds have been demonstrated in multiple tissues including the heart, brain and pancreas $[7,10,89,90]$. Here we have not only shown that AKAP7 $\alpha$ can amplify and accelerate PKC signaling, we have also provided a mechanistic explanation of how amplification and acceleration occur. In developing the scaffold state-switching model, we have generated an important tool for understanding how different scaffolds may uniquely modulate signaling enzymes by providing more or less acceleration, amplification, and insulation.

While others have reported insulation created by scaffolds, the scaffold state-switching model provides new evidence that insulation can result directly from scaffold tethering and the resulting enhanced rate of active intermediate formation. AKAP5, for example, reduces the potency of certain ATP-competitive PKC inhibitors by competing for access to the substrate-binding pocket of the enzyme [26]. In contrast, here we provide strong evidence that the insulation of $\mathrm{PKC}$ by $\mathrm{AKAP} 7 \alpha$ is an emergent property of anchoring, which is consistent with our previous observation that AKAP7 does not inhibit PKC activity [86, 88]. This insulation is distinct from the substrate specificity by scaffold tethering [83], as 
substrate specificity would have predicted reduced potency only for the substrate-competitive inhibitor. Insulation of PKC from both ATP- and substrate-competitive inhibitors indicate that insulation is a native property of scaffold tethering. Further, the scaffold state-switching model provides a biophysical explanation of how tethering alone can cause insulation on protein scaffolds.

The scaffold state-switching model differs from previous approaches used to model signaling on protein scaffolds. Levchenko et al. and O'Shaunessy et al. developed computational models to examine the role of scaffolds in MAPK signaling [52,53]. By assuming that the scaffold fixes the enzyme and substrate in an active intermediate state, they predicted amplification and acceleration of the MAPK pathway. However, such an approach cannot predict insulation from competing substrates as shown here with the scaffold state-switching model. Saucerman and colleagues assumed that AKAPs increase the local concentration of substrates ten-fold $[45,49]$ based on kinetic experimental data of tethered PKA [27]. But their phenomenological models of AKAPs implicitly assumed enhanced association rates in solution rather than mechanistically representing the "solid-state" transitions [91] within a protein complex as done in the current study. Others have modeled signaling on scaffolds using spatially explicit stochastic algorithms, which can add further detail of cellular anatomy $[54,92]$. Yet these models again either assumed that the scaffold fixed enzyme and substrate in an active intermediate state [54] or enhanced association rates due to high local concentrations [92]. Thus, the scaffold state-switching model has advantages in its mechanistic representation of reactions on a scaffold as well as the prediction of insulation from competing substrates.

In some instances, the relative stoichiometry of scaffold to enzyme or substrate can enhance or suppress signaling, an effect termed combinatorial inhibition [52,53] . Given the high affinity interaction between PKC and AKAP7 $\alpha$ [86], and the physical linkage of AKAP7 $\alpha$ to 
CKAR, we assumed that the scaffold was fully occupied by enzyme and substrate. However, future extensions of this model exploring other scaffold enzyme permutations may require the incorporation of enzyme or substrate dissociation from the scaffold. For example, phosphorylation of AKAP-Lbc can lead to a decreased association rate of Protein Kinase D (PKD) for the scaffold which increases phosphorylation of PKD by tethered PKC through substrate turnover [30]. Additionally, some scaffolds can directly alter the activity of bound enzymes [93]. AKAP5 inhibits PKC by tethering to PKC's catalytic pocket [26]. The yeast MAPK scaffold Ste5 directly increases the catalytic rate constant of Ste7 phosphorylation of Fus3 independent of tethering [94]. However, direct effects of AKAP7 on PKC have not been documented. Thus, models of other scaffolds may require consideration of both tethering and direct enzyme regulation.

Collectively, the scaffold state-switching model and its experimental validation shed light on the biophysical underpinnings of anchored enzymatic reactions that extend beyond PKC and AKAP7 $\alpha$. Our work has shown how amplification, acceleration and insulation arise from tethering reactions to scaffold proteins. The wide range of scaffold-enzyme-substrate complexes surveyed in our sensitivity analysis suggests that our findings are broadly applicable, and therefore of great interest for many clinical and bioengineering applications. Despite uncertainty in specific kinetic parameters, the model was able to predict a range of experimental data, giving us confidence in its validity. Our mechanistic representation of enzyme-scaffold dynamics provides a quantitative definition of the anchoring hypothesis. Future extensions of this theoretical and experimental framework will allow analysis of more complex signalosomes, and further our understanding of how cells use promiscuous enzymes to make specific decisions. 


\section{Chapter 5}

\section{AKAP5 coordinates both in- and out-of-phase PKA and $\mathrm{Ca}^{2+}$ oscillations}

The following people contributed to the work in this chapter: E.C. Greenwald, S. Mehta, B. Tenner., J.J. Saucerman, J. Zhang

Author contribution: E.C. Greenwald - Computational model design and development, analyzed data, wrote the chapter. S. Mehta and B. Tenner. - Performed experiments, designed experiments, analyzed data, assisted in writing the chapter. J. Zhang and J.J. Saucerman - Concieved the study, designed experiments, assisted in writing the chapter. 


\section{$5.1 \quad$ Foreword}

Now that we have described the effect of scaffold tethering on an individual reaction in

Chapter 4, we turn to study the effects of scaffolds bringing together specific networks of signaling proteins. Combinations of certain signaling connections can create signaling motifs that have been shown to lead to the emergence of complex signaling dynamics utilized throughout the body [35]. Since scaffold proteins have been shown to assemble these network motifs $[24,36,37]$, scaffold proteins have been hypothesized to play an integral role in the development of complex signaling dynamics by anchoring a specific set of signaling proteins and coordinating their interaction. In this chapter, we study the role of AKAP5 in $\mathrm{Ca}^{2+}$ and PKA oscillatory signaling as a specific example of a scaffold protein coordinating multiple signaling pathways and the feedback loops that allow crosstalk between them.

\subsection{Introduction}

Spatial and temporal coordination of signaling molecules is essential throughout biology. This organization is necessary for the development of complex signaling dynamics such as the calcium oscillations seen in cardiac [95], neuronal [96] and pancreatic [21] tissues. Tissues can coordinate signal transduction through scaffold proteins, which are proteins that bind two or more signaling proteins, tethering them together in space and time [84]. While several scaffolds have been shown to play a key role in the regulation of calcium oscillations in the heart [6] and brain [89], the role of scaffold proteins in pancreatic $\beta$-cell calcium oscillations are unclear.

Pancreatic $\beta$-cells have been shown to exhibit $\mathrm{Ca}^{2+}$ oscillations in response to glucose uptake, and these oscillations are critical for the pulsatile exocytosis of insulin [97]. Glycolysis 
stimulates these oscillations by depolarizing the plasma membrane through the inhibition of ATP sensitive potassium channels. This depolarization induces opening of the voltage gated calcium channel $\mathrm{Ca}_{\mathrm{V}} 1.2$ and the subsequent Calcium Induced Calcium Release(CICR) results in a large spike in cytosolic $\mathrm{Ca}^{2+}[98,99]$. Similar calcium oscillations in MIN6 cells, a pancreatic $\beta$-cell line, have been observed in response to glucose [97] or the potassium channel blocker tetraethylammonium chloride (TEA) $[21,100]$. While sustained $\mathrm{Ca}^{2+}$ oscillations have been studied in other cell types [95], the regulation of these glucose inducible $\mathrm{Ca}^{2+}$ dynamics in $\beta$-cells is unclear.

$\mathrm{Ca}_{\mathrm{V}} 1.2$, an essential protein in these calcium oscillations, is regulated through phosphorylation by Protein Kinase-A (PKA), where phosphorylation promotes a greater flux of $\mathrm{Ca}^{2+}$ through the channel $[101,102]$. Previously, we have shown that PKA activity is necessary for calcium oscillations in MIN6 cells and that cytosolic and plasma membrane PKA activity oscillates out-of-phase with TEA-induced $\mathrm{Ca}^{2+}$ oscillations [21]. While previous computational models have shown that the $\mathrm{Ca}^{2+}$-activated phosphodiesterase 1 (PDE1) can lead to the observed out-of-phase PKA oscillations [21,103], the coordination of the positive feedback of this oscillatory PKA activity on $\mathrm{Ca}_{\mathrm{V}} 1.2$ is unknown.

PKA signaling is coordinated by a family of scaffold proteins called A-Kinase Anchoring Proteins, AKAPs, [84] which promote the interaction of tethered signaling proteins and lead to amplification or acceleration of signal transduction [104]. The promotion of Cav1.2 regulation by PKA has been hypothesized to be a key role for AKAP5 (also known as AKAP79 or AKAP150) in the brain and heart because AKAP5 binds both Cav1.2 and PKA [102]. This AKAP also regulates the activation of PKA by associating with several different isoforms of adenylyl cyclase, AC, which catalyzes the conversion of ATP into the activator of PKA, cyclic adenosine monophosphate, cAMP [105]. Understanding the role of scaffold proteins in the regulation of these calcium dynamics will provide insight into the 
mechanisms that promote the coordination of complex signaling dynamics.

Here we study the role of AKAP5 in both the coordination of PKA dynamics and the regulation of $\mathrm{Ca}^{2+}$ oscillations by PKA. Through genetically encoded fluorescent biosensors we show that scaffold tethering is important in the development of regular calcium oscillations in MIN6 cells. Additionally, these biosensors reveal that AKAP5 tethered PKA oscillates in-phase with calcium, in contrast with the out-of-phase PKA oscillations at the plasma membrane. We developed computational models to explain the compartmental difference in PKA oscillations and found that differences between adenylyl cyclase isoform tethering can lead to simultaneous in- and out-of-phase PKA oscillations. This work identified the ability of AKAP5 to generate simultaneous, diametrically opposed dynamics in two compartments of the cell, thus encoding multiple outcomes from a single signaling pathway.

\subsection{Methods}

\section{Plasmid construction}

To generate the leucine-zipper (LZ) construct, DNA encoding a 52-amino-acid fragment that spans the LZ-like motif from Cav1.2 (GAQGRQFHGSASSLVEAVLISEGLGQFAQDPKFIEVTTQELADACDLTIEEM), which has been shown to contribute to AKAP-binding [106], was PCR-amplified using BglII and EcoRI linkers and cloned into a BglII/EcoRIdigested pcDNA3 (Invitrogen) backbone containing an HA tag followed by mCherry, a red fluorescent protein. The resulting plasmid was digested with BamHI and EcoRI to liberate the HA-mCherry-Cav1.2[LZ] fragment, which was then ligated into a BamHI/EcoR1digested pcDNA3 backbone that encodes the N-terminal 30 amino acids from DAKAP1 [65] for targeting to the outer mitochondrial membrane. For AKAP79-AKAR4, the Kozak se- 
quence, His tag, and full-length coding sequence of wild-type AKAP79 (minus the stop codon) were PCR-amplified from pcDNA3-AKAP79 (gift of John Scott) [107] using HindIII and BamHI linkers and inserted in-frame at the 5' end of AKAR4 [108] in pcDNA3.

\section{Cell culture and transfection}

MIN6 $\beta$-cells were cultured in DMEM containing $4.5 \mathrm{~g} / \mathrm{L}$ glucose and supplemented with $10 \%$ (v/v) FBS, $1 \%$ (v/v) Pen-Strep, and $50 \mu \mathrm{M} \beta$-mercaptoethanol. All cells were maintained in a humidified incubator at $37^{\circ} \mathrm{C}$ with a $5 \% \mathrm{CO}_{2}$ atmosphere. Prior to transfection, cells were plated onto sterile, 35-mm glass-bottomed dishes and grown to 50-70\% confluence. Cells were then transfected using Lipofectamine 2000 (Invitrogen) and cultured an additional 48 hours before imaging.

\section{Imaging}

Cells were washed with Hank's Balanced Salt Solution (HBSS) and subsequently imaged in the dark at $37^{\circ} \mathrm{C}$. Tetraethylammonium chloride (TEA; Sigma), St-Ht31 (Promega), and St-Ht31p (Promega) were added as indicated. Images were acquired using an Axiovert 200M inverted fluorescence microscope (Carl Zeiss, Thornwood, NY) with a 40x/1.3 NA oil-immersion objective lens and a cooled charge-coupled device camera (Roper Scientific, Trenton, NJ) controlled by Metafluor 7.7 software (Molecular Devices, Sunnyvale, CA). Fura-2 dual excitation ratio imaging was performed using two excitation filters (350DF10 for $350 \mathrm{~nm}$ excitation and 380DF10 for $380 \mathrm{~nm}$ excitation), a 450DRLP dichroic mirror and a 535DF45 emission filter. Dual cyan/yellow emission ratio imaging was performed using a 420DF20 excitation filter, a 450DRLP dichroic mirror, and two emission filters (475DF40 for CFP and 535DF25 for YFP). RFP intensity was imaged using a 568DF55 excitation 
filter, a 600DRLP dichroic mirror, and a 653DF95 emission filter. Filter sets were alternated using a Lambda 10-2 filter changer (Sutter Instruments, Novato, CA). Exposure times were between 10 and $500 \mathrm{~ms}$, and images were taken every 20-30 s. Raw fluorescence images were corrected by subtracting the background fluorescence intensity of a cell-free region from the emission intensities of biosensor-expressing or Fura-loaded cells. Emission ratios (yellow/cyan or F350/F380) were then calculated at each time point. All time-courses were normalized to the basal emission ratio or intensity, in the case of RCaMP, immediately before drug addition.

\section{AKAP disruption experiments}

For global AKAP disruption experiments, MIN6 cells transfected with RCaMP were pretreated with $5 \mu \mathrm{M}$ St-Ht31 or St-Ht31p for 30 min at $37^{\circ} \mathrm{C}$ prior to imaging. For targeted disruption of the AKAP79-Cav1.2 interaction, MIN6 cells transfected with the mCherry$\mathrm{Ca}_{\mathrm{V}} 1.2[\mathrm{LZ}]$ construct were loaded with $1 \mu \mathrm{M}$ Fura-2/AM for $10 \mathrm{~min}$ at $37^{\circ} \mathrm{C}$ prior to imaging. During analysis, the cells were then divided into two groups: cells with a backgroundsubtracted RFP intensity $>1000$ were considered $\mathrm{Ca}_{\mathrm{V}} 1.2[\mathrm{LZ}]$-expressing cells, and cells with a background-subtracted RFP intensity $<500$ were considered untransfected and used as an internal control. Cells with intermediate intensity were excluded from the analysis. Cellular $\mathrm{Ca}^{2+}$ responses in response to TEA treatment for the control and AKAP disrupted cells were classified as either regular or irregular oscillatory behavior. These classifications were performed in a blinded and randomized fashion by different observers and repeated with 3 technical repeats. Differences between observers precluded the ability to combine classifications between observers but the same trends were seen by all observers. 


\section{Model Development}

Computational models were developed to describe compartmentalized PKA activity, $\mathrm{Ca}^{2+}$ dynamics and cellular electrophysiology in MIN6 $\beta$-cells and implemented in MATLAB (MathWorks). These models have 3 interconnected modules of systems of Ordinary Differential Equations (ODEs): Electrophysiology/ $\mathrm{Ca}^{2+}$, Calmodulin (CaM) and PKA modules. The Electrophysiology $/ \mathrm{Ca}^{2+}$ module is derived from the previously developed $\beta$-cell model by Fridlyand et al., which is able to replicate TEA-induced $\mathrm{Ca}^{2+}$ oscillations [99]. This module has states for the cellular membrane voltage (V) and cytosolic $\mathrm{Ca}^{2+}$ concentration ( $\mathrm{Ca}_{i}$ ) that are dependent on $\mathrm{K}^{+}, \mathrm{Na}^{+}$, and $\mathrm{Ca}^{2+}$ membrane channel fluxes. The CaM module is derived from a previous model by Saucerman and Bers and contains states for CaM that has four, two or no bound calcium, where the four $\mathrm{Ca}^{2+} \mathrm{CaM}$ is assumed to be the active state [109]. Finally, the PKA modules originated from our previous model of $\beta$-cell PKA activity [21] and then was expanded using reactions developed based on experimental evidence and previous models $[45,63,104,110,111]$. This module contains two isolated compartments for the plasma membrane and AKAP5 nanodomain. This module models PKA activation by cAMP, and the production and degradation of cAMP by adenylyl cyclase and phosphodiesterases, respectively. Additionally, phosphorylation of the FRET biosensor for PKA activity, AKAR, was incorporated into the model. Model parameters were defined using literature values of biochemical rate constants. A detailed description of the model equations and parameters is given in the Appendix B.

\section{Statistics}

Comparisons of the percent of cells exhibiting regular oscillations were performed using the hypothesis test for the equality of two binomial proportions in MATLAB. The average 
number of regularly oscillating cells across 3 technical repeats for a single observer was used for this statistic. Unpaired and unequal variance t-tests for the time lag quantification were performed in MATLAB. $\mathrm{p}<0.05$ was considered significant.

\subsection{Results}

\subsubsection{Disruption of AKAP5 or PKA tethering decreases regular TEA induced calcium oscillations}

To investigate the role of scaffold tethering in MIN6 $\mathrm{Ca}^{2+}$ oscillations we disrupted the binding of Cav1.2 to AKAP5 or PKA tethering to AKAPs. Live cell $\mathrm{Ca}^{2+}$ dynamics in MIN6 cells were measured with the genetically encoded fluorescent $\mathrm{Ca}^{2+}$ biosensor RCaMP or the cell permeable $\mathrm{Ca}^{2+}$ dye Fura-2/AM [112]. Induction of $\mathrm{Ca}^{2+}$ oscillations in Fura-2/AM loaded MIN6 cells by the $\mathrm{K}^{+}$channel blocker TEA led to sustained oscillations in the Fura2/AM fluorescence intensity, consistent with our previous studies [21, 100] (Figure 5.1A). We evaluated the impact of disrupting the AKAP5-Cav1.2 tethering on TEA-induced $\mathrm{Ca}^{2+}$ oscillations by expressing a leucine zipper peptide (LZ) that competes for the Cav1.2 binding domain on AKAP5 [113]. Cells expressing this LZ peptide exhibited more disrupted or decaying oscillations (Figure 5.1B). Blinded observers classified $\mathrm{Ca}^{2+}$ oscillations from both control and AKAP5 disrupted cells as either regular or irregular. Indeed, expression of the LZ peptide led to a significant decrease in the percentage of cells exhibiting regular oscillations in response to TEA treatment (Figure 5.1C). Similarly, disrupting general PKA tethering to AKAPs by pre-treating RCaMP transfected MIN6 cells with St-Ht31, a membrane-permeable peptide that competes for the binding site of PKA on AKAPs, also decreased the fraction of cells that oscillate regularly in response to TEA compared to the 
phosphorylated control St-Ht31p, that does not compete for PKA binding to AKAPs (Figure 5.1C). These data show that both AKAP5 binding to $\mathrm{Ca}_{\mathrm{V}} 1.2$ and AKAP-tethering of PKA mediate the formation of regular TEA-induced $\mathrm{Ca}^{2+}$ oscillations in MIN6 cells.

\subsubsection{AKAP5-tethered PKA oscillates in-phase with $\mathrm{Ca}^{2+}$}

Our previous work showed TEA-induced $\mathrm{Ca}^{2+}$ coordinates out-of-phase oscillations in cytosolic and plasma membrane PKA activity [21]. We hypothesized that in addition to promoting the regulation of $\mathrm{Ca}_{\mathrm{V}} 1.2$ by PKA, AKAP5 may modulate the activation of PKA by $\mathrm{Ca}^{2+}$. Simultaneous measurement of $\mathrm{Ca}^{2+}$ concentration and plasma membrane PKA activity within single cells were performed by co-expressing RCaMP and the plasma membrane-targeted FRET-biosensor of PKA activity, Lyn-AKAR4, in MIN6 cells [21,27,114]. In agreement with our previous data [21], these data exhibited out-of-phase RCaMP and Lyn-AKAR4 oscillations in response to TEA stimulation (Figure 5.2A). To quantify the effect of AKAP5 tethering on PKA activity we genetically fused the PKA biosensor AKAR4 with AKAP5 and co-expressed this AKAP5-AKAR4 fusion protein with RCaMP in MIN6 cells. In contrast to the out-of-phase Lyn-AKAR4 oscillations, TEA stimulated cells expressing AKAP5-AKAR4 exhibited oscillations in-phase with RCaMP (Figure 5.2B). Quantification of these phase dynamics shows that Lyn-AKAR consistently exhibited a significant temporal lag in both the PKA peak and transient initiation to that of $\mathrm{Ca}^{2+}$, whereas in AKAP5-AKAR4 the transient initiation and peaks of PKA and $\mathrm{Ca}^{2+}$ occurred nearly simultaneously (Figure 5.3). These novel, contrasting dynamics occurring at the plasma membrane and in the AKAP5 nanodomain are unique evidence of scaffold proteins promoting compartmentalized signaling dynamics. 
A

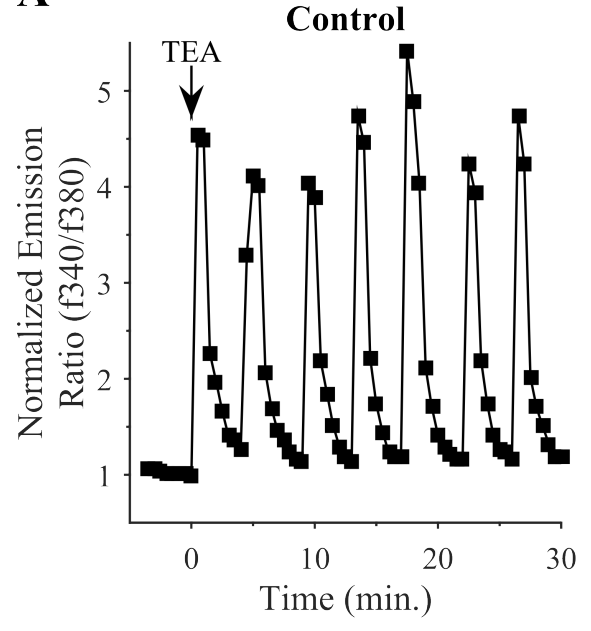

B

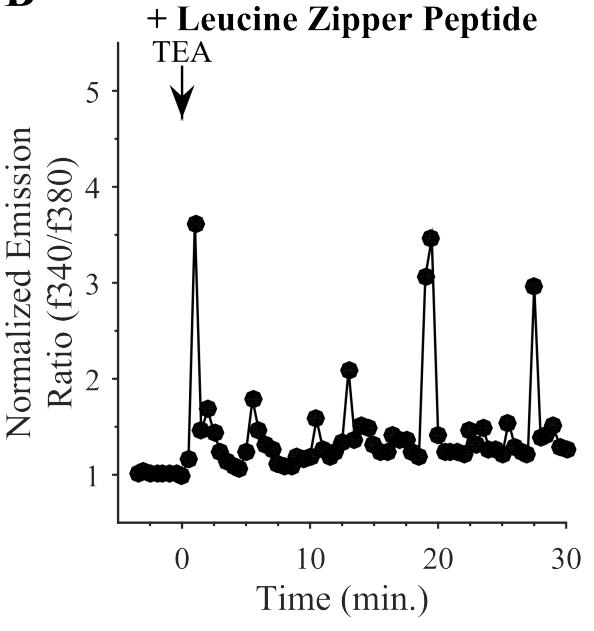

C

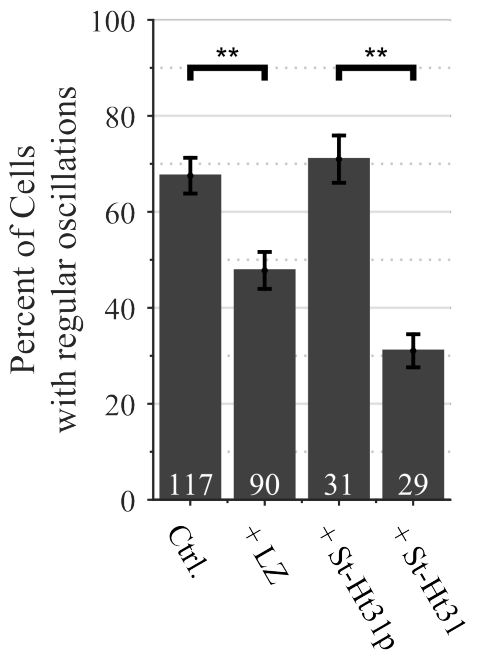

Figure 5.1: Disruption of scaffold tethering reduces regular $\mathrm{Ca}^{2+}$ oscillations. Representative single cell traces of TEA-induced calcium oscillations, measured by Fura-2/AM fluorescence, for control (A) or Leucine zipper peptide co-transfected (B) cells. C) Expression of the leucine zipper peptide and treatment with HT31 reduced the percentage of cells exhibiting regular Fura-2/AM or RCaMP fluorescence oscillations compared to control and HT31p treated cells, respectively. Error bars are standard deviation across technical replicates for a single blinded observer $(\mathrm{n}=3)$. Statistics between groups were performed using the number of cells in each condition, indicated at the bottom of the bar, and performed using the hypothesis test for the equality of two binomial proportions. ** $\mathrm{p}<0.01$ 
A

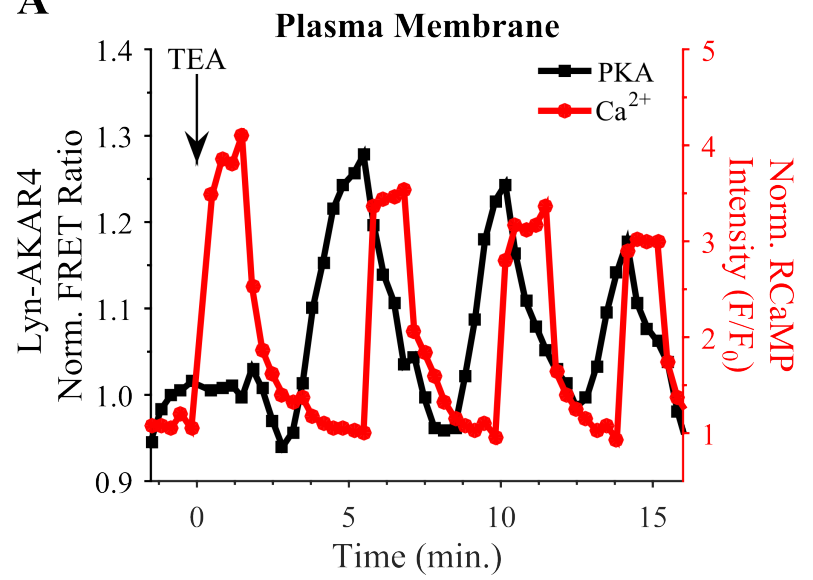

B

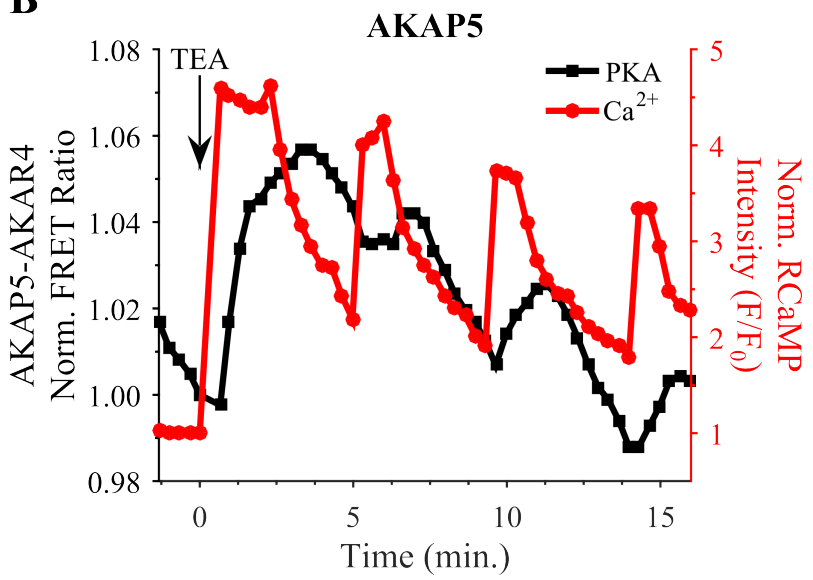

Figure 5.2: AKAP5-tethered PKA activity oscillates in-phase with $\mathrm{Ca}^{2+}$. Simultaneous measurement of calcium concentration (Red) and either plasma membrane (A) or AKAP5-tethered (B) PKA activity (black) by co-expression of RCaMP and Lyn-AKAR4 or AKAP5-AKAR4, respectively. Representative single cell traces display $\mathrm{PKA}$ and $\mathrm{Ca}^{2+}$ oscillations that are out-of-phase at the plasma membrane (A) but in-phase on AKAP5 (B).

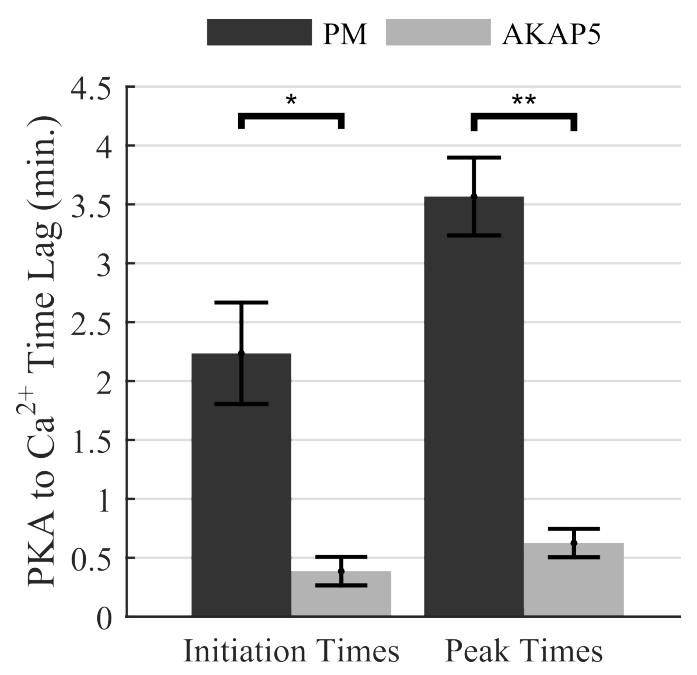

Figure 5.3: Quantification of $\mathrm{PKA}$ to $\mathrm{Ca}^{2+}$ temporal lag. The time difference for transient initiation (initiation times) and transient peaks (peak times) between the RCaMP and either LynAKAR4 (black bars, $n=3$ ) or AKAP5-AKAR4 (gray bars, $n=5$ ) were quantified for each cell. These metrics indicate a significantly longer lag for plasma membrane PKA activity than for AKAP5tethered PKA. ${ }^{*} \mathrm{p}<0.05,{ }^{* *} \mathrm{p}<0.01$, Error bars represent s.e.m. 


\subsubsection{Model requires $\mathrm{Ca}^{2+}$ induced activation of PKA to agree with experimental data}

The unique nature of PKA oscillations that are simultaneously in- and out-of-phase with calcium in two different cellular compartments suggests that there is a complex coordination of several feedback mechanisms. To evaluate competing hypotheses for these mechanisms, we developed several computational biochemical Ordinary Differential Equation (ODE) models of MIN6 electrophysiology, calcium dynamics, and PKA regulation. The asynchronous nature of oscillations in individual MIN6 cells in response to TEA prevents averaging of $\mathrm{Ca}^{2+}$ and PKA dynamics across cells. Therefore, we defined the following qualitative features seen experimentally in all cells and evaluated the extent to which the computational models exhibited these features (Table 5.1):

(1) Plasma membrane PKA activity increases after the $\mathrm{Ca}^{2+}$ concentration decreases.

(2) Plasma membrane PKA activity shows a sharp decrease that is coincident with the initiation of the $\mathrm{Ca}^{2+}$ transient.

(3) PKA phosphorylation increased from baseline after TEA stimulation for both the plasma membrane and AKAP5 compartments.

(4) Plasma membrane PKA exhibits larger oscillations than AKAP5-tethered PKA.

(5) AKAP5-tethered PKA oscillation initiation occurs with the initiation of the $\mathrm{Ca}^{2+}$ transient.

The evaluation of different hypothesized mechanisms for these features, as referenced by the above circled numbers for the rest of the chapter, allowed us to critically evaluate the phenotypic outcomes of different models. 
We first examined the mechanisms that underlie the regulation of PKA by $\mathrm{Ca}^{2+}$. To model $\mathrm{Ca}^{2+}$ dynamics in MIN6 cells, we used a previously developed model of $\beta$-cell electrophysiology and $\mathrm{Ca}^{2+}$ dynamics (EP model) [99]. This EP model is able to provide a good approximation the TEA-induced $\mathrm{Ca}^{2+}$ oscillations [99] but does not directly incorporate PKA activity. Consequently, this EP model de-couples the regulation of $\mathrm{Ca}^{2+}$ oscillations and PKA activity from each other. With this EP model we were able to test hypothesized mechanisms by which $\mathrm{Ca}^{2+}$ can regulate PKA without having to simultaneously consider the positive feedback of PKA on the Cav1.2 channel. Therefore, we first developed ODE models that contain separated modules for $\mathrm{Ca}^{2+}$ and PKA activity and incorporated mechanisms by which $\mathrm{Ca}^{2+}$ may drive PKA activity (Appendix B)

Our previous work identified that the $\mathrm{Ca}^{2+}$ activated PDE1 is a primary driver of outof-phase PKA oscillations observed at the plasma membrane [21]. We incorporated this mechanism of $\mathrm{Ca}^{2+}$-dependent regulation of PKA activity into the EP model to examine the effect of this $\mathrm{Ca}^{2+}$ driven approach on our previously published model of $\beta$-cell PKA dynamics [21] (Figure 5.4A, described in detail in Appendix B). This "Ni 2011" model still exhibited out-of-phase PKA activity at the plasma membrane, agreeing with our qualitative features (1) and (2), but does not exhibit increases in PKA activity after TEA stimulation (feature (3)) (Figure 5.4B, Table 5.1). The Ni 2011 model is unable to capture feature (3) because this model assumed a high basal adenylyl cyclase (AC) activity and did not incorporate any mechanism for calcium stimulated increase in PKA activity.

This led us to hypothesize that the calcium activated adenylyl cyclases, AC1 and AC8 [115], may be important for the calcium dependent increase in PKA activity. While both AC1 and AC8 are expressed in pancreatic $\beta$-cells [116] and MIN6 cells [117], AKAP5 binds AC8 but not AC1 [105]. Thus, we assumed that AC8 was primarily responsible for the $\mathrm{Ca}^{2+}$ activation of PKA in TEA-stimulated $\mathrm{Ca}^{2+}$ oscillations. With $\mathrm{Ca}^{2+}$ simultaneously 
activating PDE1 and AC8, merely adding AC8 to our previous model would not lead to $\mathrm{Ca}^{2+}$ stimulated and out-of-phase PKA oscillations. Indeed, a previous model [103] that did incorporate both the $\mathrm{Ca}^{2+}$ activated $\mathrm{AC} 8$ and PDE1 found that a high basal adenylyl cyclase activity was needed for out-of-phase PKA oscillations in their model. But this high basal adenylyl cyclase activity exhibited out-of-phase PKA oscillations due to decreases in PKA activity from baseline, similar to our Ni 2011 model. Thus, additional mechanisms are required to obtain both out-of-phase oscillations and an increase in PKA activity after TEA stimulation (feature (3)).

\subsubsection{Diffusion of cAMP between compartments is insufficient for robust plasma membrane PKA oscillations}

To be able to capture both an in-phase PKA activity localized to AKAP5 and an out-of-phase PKA activity at the plasma membrane we assumed that there are two insulated compartments, AKAP5 and plasma membrane. In the AKAP5 compartment, we hypothesized that the in-phase PKA activity is driven by the $\mathrm{Ca}^{2+}$ induced activation of $\mathrm{AC} 8$ and not impeded by PDE1, since AKAP5 has been shown to bind AC8 $[118,119]$ but no evidence for PDE1 binding has been shown. While incorporation of AC8 into the model can lead to in-phase

PKA activity at AKAP5 (feature (5), this does not address the combination of out-of-phase oscillations and TEA-induced increase in PKA activity at the plasma membrane (features (1) - (3).

One hypothesis for the delayed activation of plasma membrane PKA is that diffusion of cAMP produced by the AKAP5-tethered AC8 induces a significant delay such that plasma membrane PKA is out-of-phase [120]. To test this diffusion hypothesis, we developed a "cAMP Diffusion Model" where cAMP is produced by AKAP5-tethered AC8 and cAMP is 
allowed to diffuse between the AKAP5 and plasma membrane compartments (Figure 5.5A). The inclusion of AC8 in the AKAP5 compartment did indeed lead to in-phase PKA activity at AKAP5 (Figure 5.5C). Furthermore, the combination of this $\mathrm{Ca}^{2+}$ induction of cAMP and PDE1 did allow PM PKA activity to be out-of-phase and increase from baseline but the magnitude of the oscillations were very small due to the rapid decrease in cAMP in the AKAP5 compartment after the $\mathrm{Ca}^{2+}$ decreases (Figure 5.5B). Thus, the cAMP diffusion

model does not capture feature (4), which is indicative of the fact that these AKAP complexes form nanodomains that may not be able to supply enough cAMP to elicit large enough responses at the plasma membrane (Table 5.1). The cAMP Diffusion Model provides insight that the tethered AC8 is sufficient to create the in-phase PKA oscillations on AKAP5, but diffusion of cAMP out of this compartment is insufficient to produce robust PKA oscillations at the plasma membrane.

\subsubsection{Differences in phosphatase concentration inconsistent with sharp decrease in PKA activity}

An alternative hypothesis is that the measured differences in PKA phase are due to compartmental differences in the biosensor and phosphatase concentrations. In this "Phosphatase Model", we assumed that AC8 was the source of cAMP for both compartments and allowed the biosensor and phosphatase concentration to differ between compartments (Figure 5.6A). We were able to identify a model that did agree with most of the experimental features, but the plasma membrane PKA oscillations did not exhibit the characteristic sharp decrease that is coincident with the next $\mathrm{Ca}^{2+}$ oscillation (Figure 5.6B). The AKAP5 PKA activity in the Phosphatase Model is still dictated by AC8, thus it still exhibited in-phase oscillations (Figure 5.6C). The Phosphatase Model fails to produce dynamics that agree with feature 
A

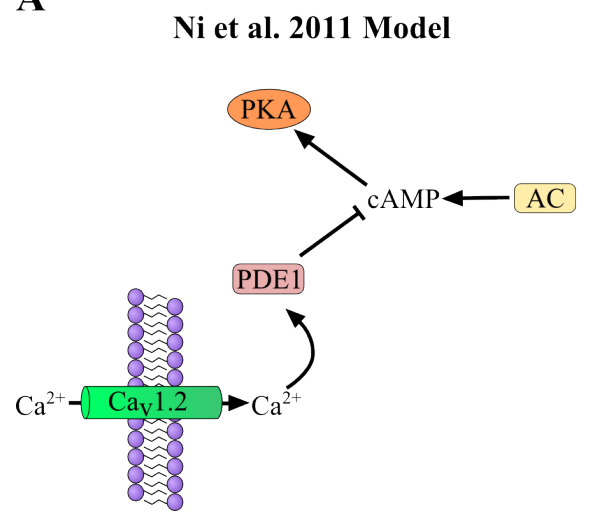

B

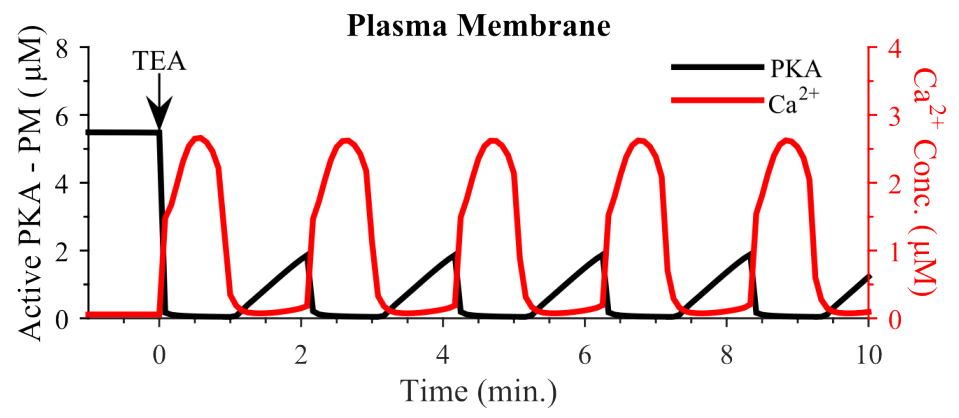

Figure 5.4: Previous model exhibits out-of-phase PM PKA oscillations that decrease from baseline. A) Simplified model diagram showing activation of PDE1 by TEA-induced $\mathrm{Ca}^{2+}$ leading to decreased in cAMP and activated PKA. B) In response to TEA stimulation the model exhibits oscillations in $\mathrm{Ca}^{2+}$ concentration (red) and out-of-phase PKA oscillations that decrease from baseline.
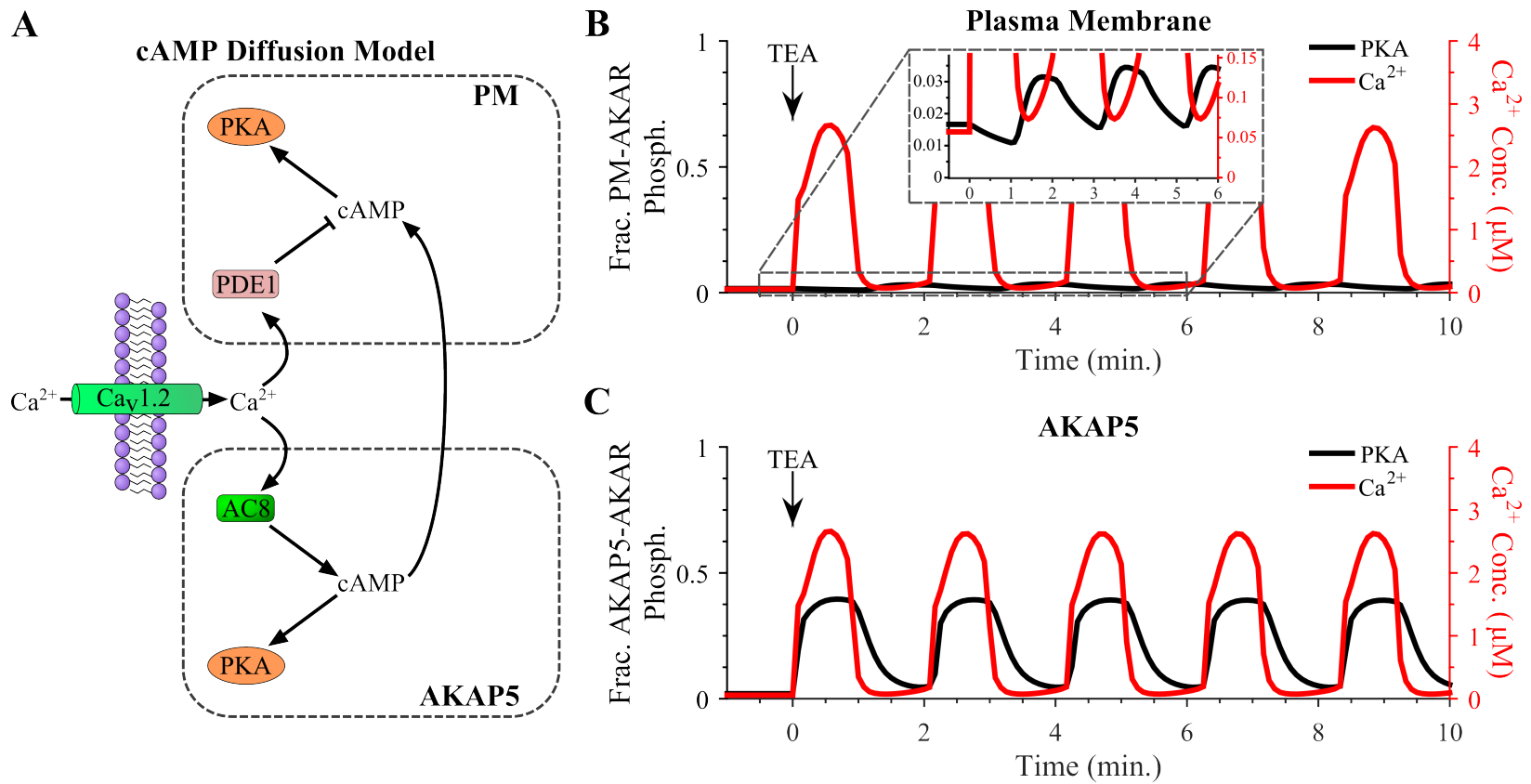

Figure 5.5: cAMP Diffusion model exhibits correct phase but small PKA dynamics. A) Simplified model diagram shows the 2 model compartments, plasma membrane and AKAP5, where PDE1 or AC8 are activated by $\mathrm{Ca}^{2+}$, respectively. cAMP is allowed to freely diffuse between the two compartments. Model simulation results of $\mathrm{Ca}^{2+}$ concentration (red) and AKAR phosphorylation (black) show small, out-of-phase oscillations at the plasma membrane (B, see inset for detail) and in-phase oscillations in the AKAP5 compartment (C). 
(2) because the phosphatase activity and concentration had to be decreased to such a level that makes the biosensor insensitive to decreases in the PKA activity (Table 5.1). A toy example of this type of biosensor kinetics based artifact can be seen in Figure 3.5. The disagreement between the Phosphatase Model and the acute sensitivity of the LynAKAR4 biosensor indicates that PDE1 activity is indeed a primary driver of the out-of-phase PKA activity and serves to assuage concerns that these dynamics are simply biosensor artifact.

\subsubsection{AKAP5-coordinated activation of AC8 and AC5 concords with all five observed oscillatory features}

One final hypothesis to explain the contrasting PKA phase dynamics is that AKAP5 coordinates the activation of two separate pools of adenylyl cyclase, where the plasma membrane adenylyl cyclase pool can be distinctly activated in a temporally delayed manner. In addition to PKA, Cav 1.2 and AC8, AKAP5 also binds Protein Kinase C, PKC, and Calmodulin, CaM [121]. At low $\mathrm{Ca}^{2+}$ concentrations, PKC binds AKAP5 tighter than CaM but activation of $\mathrm{CaM}$ by $\mathrm{Ca}^{2+}$ increases the affinity of CaM for AKAP5 and allows activated CaM to displace PKC $[121,122]$. Furthermore, this displaced PKC would then be free to phosphorylate and activate AC5 [123]. This mechanism would thereby lead to a calcium dependent and delayed activation of AC5 driving the out-of-phase, TEA-stimulated PKA oscillations at the plasma membrane. We incorporated this hypothesized mechanism into the model by including PKC and CaM and assuming that PKC and CaM compete for a single binding site on AKAP5. Additionally, we assumed that PKC and CaM compete for this site by trading places in the plasma membrane and AKAP5 compartments via an "antiporter membrane transport protein"-like mechanism (Figure 5.7A, refer to Appendix B for details). This "AC8/AC5 Model" was able to produce both the in- and out-of-phase PKA oscillations 


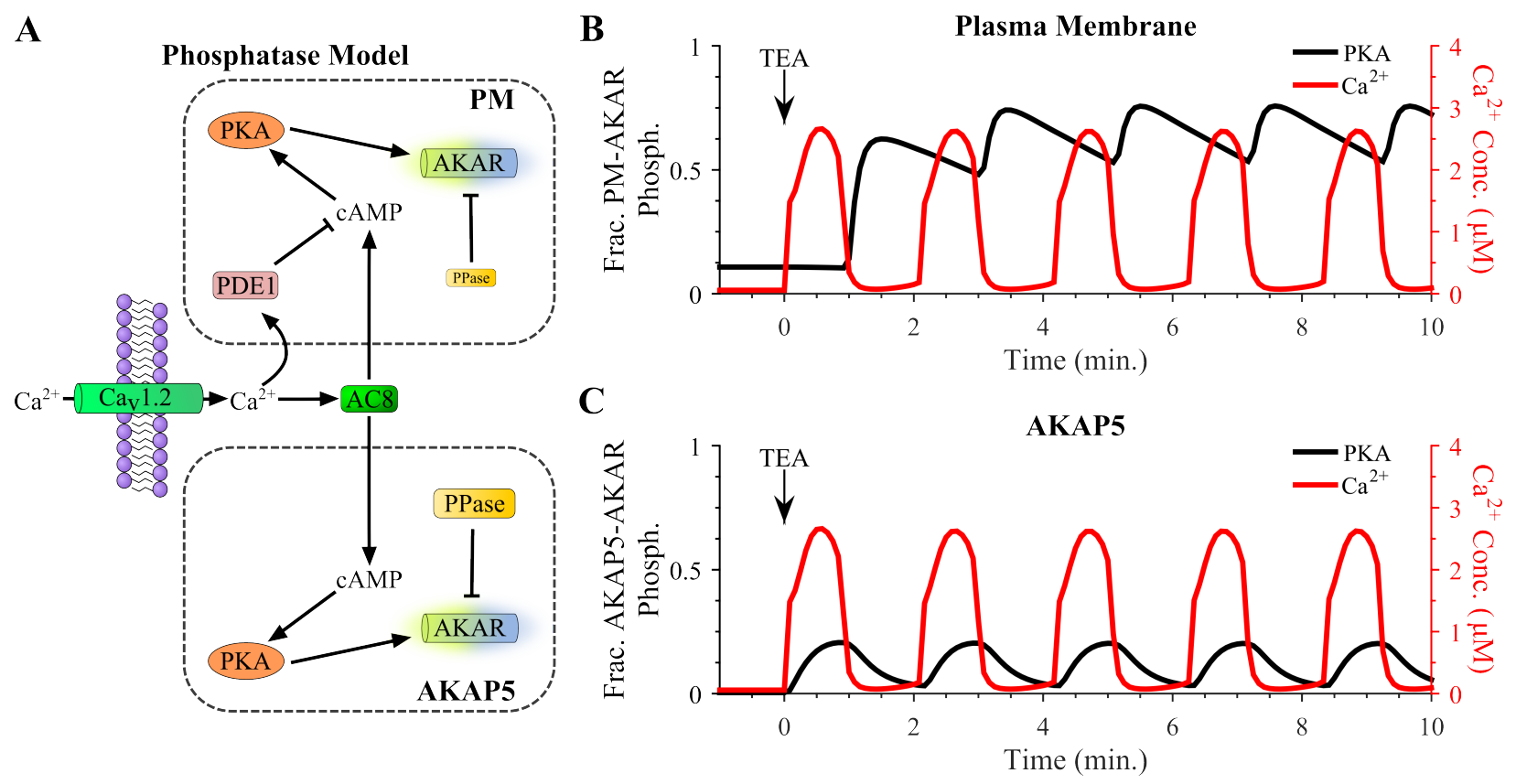

Figure 5.6: Phosphatase model oscillations are insensitive to decreases in PKA activity. A) The phosphatase model assumes that calcium activated AC8 produces cAMP for both the PM and AKAP5 compartments and that the phosphatase concentration and dephosphorylation kinetics are reduced in the PM compartment. B) This model still produced in-phase oscillations on AKAP5 (C) but lead to out-of-phase oscillations in the plasma membrane but the AKAR phosphorylation dynamics (black) are insensitive to decreases in the PKA activity as seen by a lack of decrease upon the next calcium (red) spike. 
that recapitulate all five of the qualitative features of our experimental results (Figure 5.7BC). This agreement between the experimental data and this AC8/AC5 model suggests that AKAP5 may promote the development of both in- and out-of-phase PKA oscillations by coordinating the activation both $\mathrm{AC} 8$ and $\mathrm{AC} 5$ through two different mechanisms.

\subsubsection{Computational model predicts that AKAP5-tethered PKA is necessary for TEA induced calcium oscillations}

With our improved understanding of the mechanisms underlying the coordination of $\mathrm{Ca}^{2+}$ dependent PKA dynamics by AKAP5, we sought to better understand the aforementioned observation that tethering of PKA and $\mathrm{Ca}_{\mathrm{V}} 1.2$ are critical for the development regular $\mathrm{Ca}^{2+}$ oscillations (Figure 5.1). The AC8/AC5 model of simultaneous in- and out-of-phase PKA activity enables us to computationally evaluate whether the feedback of PKA onto the Cav 1.2 channel requires the in-phase, AKAP5-tethered PKA activity or the out-of-phase plasma membrane PKA. To test this we extended the AC8/AC5 model to incorporate phosphorylation of the $\mathrm{Ca}_{\mathrm{V}} 1.2$ by PKA, which leads to an approximately 2 fold increase in $\mathrm{Ca}^{2+}$ conductance through the channel [124] (Figure 5.8A). When the plasma membrane PKA activity was used for the feedback onto the $\mathrm{Ca}_{\mathrm{V}} 1.2$ channel, stimulation with TEA is insufficient to produce calcium oscillations (Figure 5.8B). When we examine the feedback of AKAP5-tethered PKA on $\mathrm{Ca}_{\mathrm{V}} 1.2$, the amplification and acceleration that can occur with scaffold tethered reactions must also be considered [84]. Previous models studying PKA phosphorylation of $\mathrm{Ca}_{\mathrm{V}} 1.2$ in cardiac cells have assumed that scaffold tethering leads to a 10-fold increase in the "effective concentration" of PKA [45,49], and for simplicity we also ap-

plied this assumption. When this AKAP5-tethered PKA activity is used for $\mathrm{Ca}^{2+}$-induced feedback onto the Cav1.2 channel, TEA stimulation leads to stable oscillations in $\mathrm{Ca}^{2+}$ 

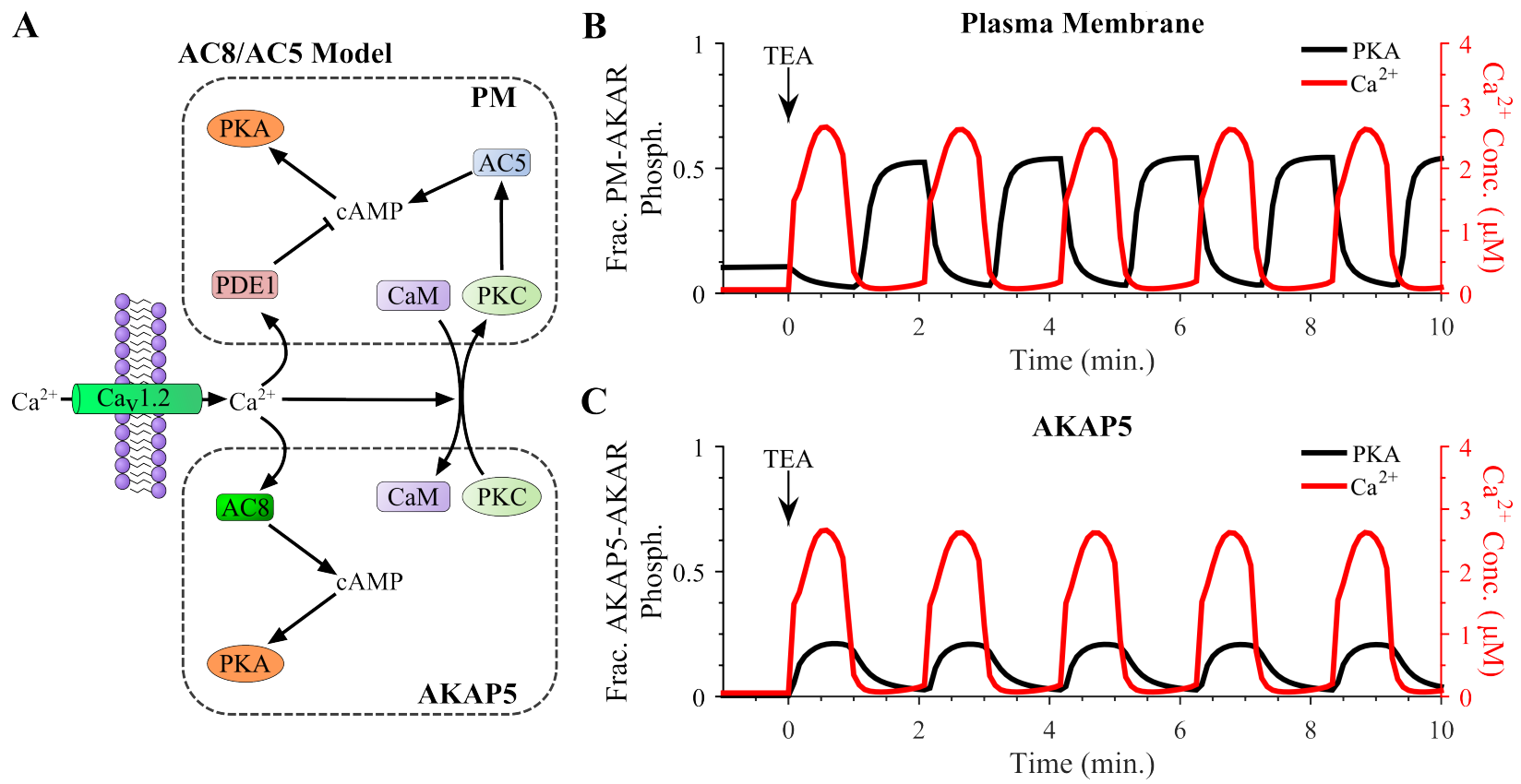

Figure 5.7: AC8/AC5 model agrees with all qualitative metrics seen experimentally. A) Simplified model schematic shows the TEA-induced $\mathrm{Ca}^{2+}$ activation of PDE1 and AC8 in the plasma membrane and AKAP5 compartments, respectively. Additionally, activation of CaM by $\mathrm{Ca}^{2+}$ causes CaM to displace PKC from the AKAP5 compartment and translocate to the plasma membrane compartment. This PKC is then able to activate AC5, thus producing cAMP. Model simulations show that the AC8/AC5 model that the $\mathrm{Ca}^{2+}$ (red) and PKA (black) oscillations are TEA-stimulated and out-of-phase at the plasma membrane (B) and in-phase on AKAP5 (C). 
(Figure 5.8C) agreeing with our experimental evidence showing that disruption of AKAP5$\mathrm{Ca}_{\mathrm{V}} 1.2$ binding or PKA tethering to AKAPs leads to a reduction in the regular oscillations in response to TEA. Thus, this extended AC8/AC5 model provides further support that the AKAP5 tethered and $\mathrm{Ca}^{2+}$ induced positive feedback by PKA onto the $\mathrm{Ca}_{\mathrm{V}} 1.2$ channel is important for the generation of oscillatory $\mathrm{Ca}^{2+}$ dynamics in MIN6 Cells.

Table 5.1: Features of Experimental PKA Oscillations. Summary of the ability of each of our models to capture observed features of PKA oscillations

\begin{tabular}{|c|c|c|c|c|c|}
\hline \multirow[b]{3}{*}{ Models } & \multicolumn{5}{|c|}{ Experimental Features } \\
\hline & \multicolumn{2}{|c|}{$\mathrm{PM}$} & \multicolumn{2}{|c|}{ PM and AKAP5 } & AKAP5 \\
\hline & $\begin{array}{c}1 \\
\mathrm{PKA} \\
\text { increase } \\
\text { after } \mathrm{Ca}^{2+} \\
\text { decrease }\end{array}$ & $\begin{array}{c}2 \\
\text { PKA } \\
\text { decrease } \\
\text { with } \mathrm{Ca}^{2+} \\
\text { increase }\end{array}$ & $\begin{array}{c}3 \\
\text { PKA } \\
\text { increase } \\
\text { from } \\
\text { baseline }\end{array}$ & $\begin{array}{c}4 \\
\text { PM - PKA } \\
\text { oscil. larger } \\
\text { than } \\
\text { AKAP5 }\end{array}$ & $\begin{array}{c}5 \\
\text { PKA } \\
\text { increase } \\
\text { with } \mathrm{Ca}^{2+} \\
\text { increase }\end{array}$ \\
\hline \multirow{4}{*}{$\begin{array}{l}\text { Ni } 2011 \\
\text { cAMP Diffusion } \\
\text { Phosphatase } \\
\text { AC5/AC8 }\end{array}$} & Yes & Yes & No & $\mathrm{N} / \mathrm{A}$ & $\mathrm{N} / \mathrm{A}$ \\
\hline & Yes & Yes & Yes & No & Yes \\
\hline & Yes & No & Yes & Yes & Yes \\
\hline & Yes & Yes & Yes & Yes & Yes \\
\hline
\end{tabular}




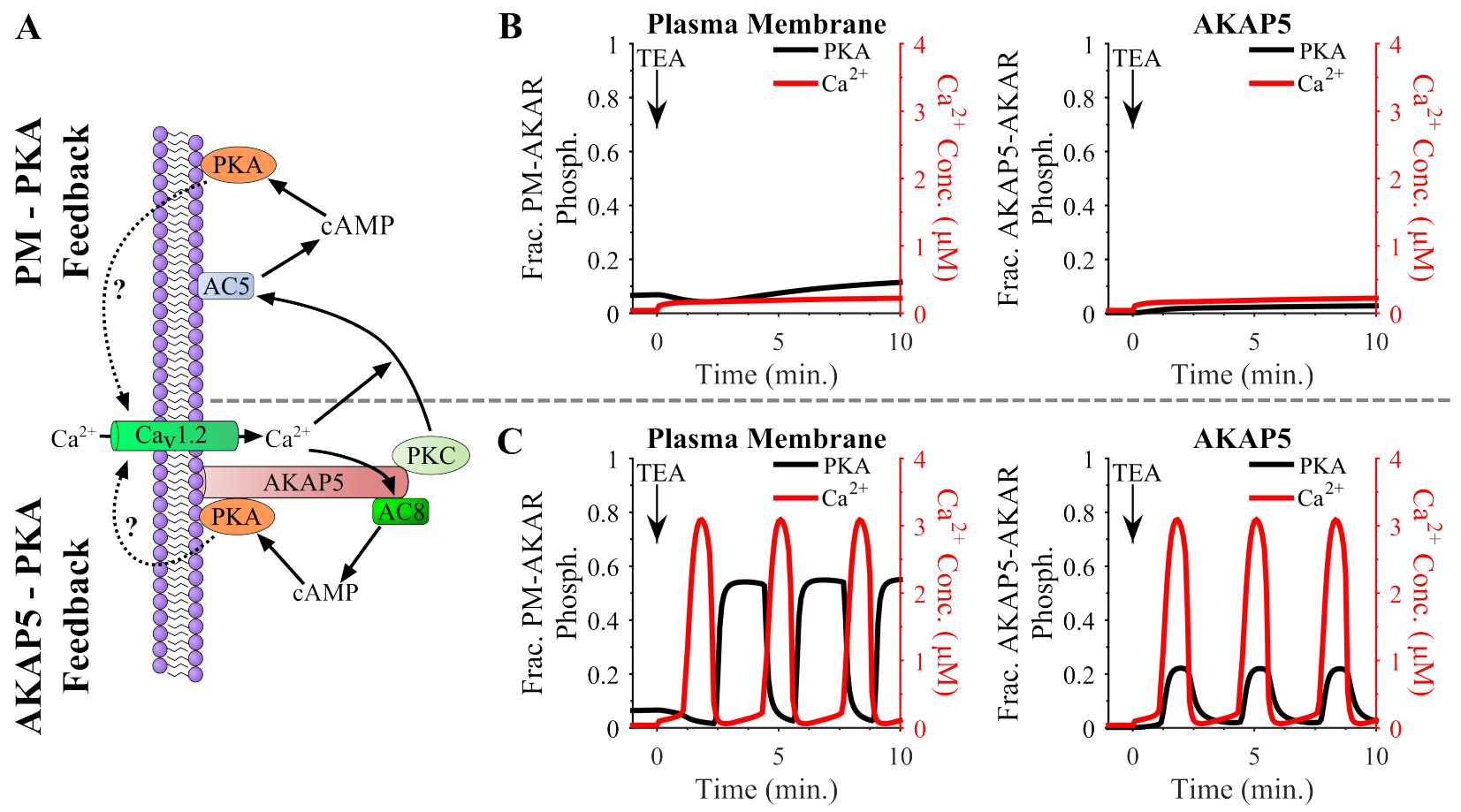

Figure 5.8: Positive feedback by AKAP5-tethered PKA necessary for TEA-induced $\mathrm{Ca}^{2+}$ oscillations. A) Simplified model diagram showing that the AC8/AC5 model was extended to include phosphorylation of Cav1.2 by either plasma membrane PKA (top) or AKAP5-tethered PKA (bottom). B) Feedback of plasma membrane PKA did not lead to oscillations in $\mathrm{Ca}^{2+}$ (red) or PKA (black) in response to TEA stimulation. C) TEA stimulation in a model with feedback by AKAP5tethered PKA produced stable $\mathrm{Ca}^{2+}$ (red) oscillations and maintained the PKA (black) phase relationships of the AC8/AC5 model. 


\subsection{Discussion}

By bringing together disparate signaling pathways, scaffold proteins are capable of coordinating crosstalk throughout the cell. Here we have shown that AKAP5 coordinates $\mathrm{Ca}^{2+}$ and PKA signaling in MIN6 cells (Figure 5.1). In addition, in- and out-of-phase PKA oscillations on AKAP5 and the plasma membrane, respectively, provides novel evidence that scaffold proteins are capable of shaping unique and compartmentalized signaling dynamics (Figures 5.2 and 5.3). The development of computational models allowed us to test several hypothesized mechanisms underlying these signaling dynamics. Through these models we identified that the $\mathrm{Ca}^{2+}$ activated AC8 is responsible for the in-phase PKA oscillations on AKAP5. Additionally, the coordination of PKC by AKAP5 is sufficient to initiate delayed activation of AC5 at the plasma membrane leading to stimulated and out-of-phase PKA oscillations (Figure 5.7). This novel mechanism improves upon previous models [21,103] because it captures both the $\mathrm{Ca}^{2+}$ stimulated increase in plasma membrane PKA activity and its antiphase relationship with $\mathrm{Ca}^{2+}$. Finally, our computational model provided evidence that the immediately activated and AKAP5 tethered PKA positive feedback is necessary for the generation of TEA induced $\mathrm{Ca}^{2+}$ oscillations (Figure 5.8).

AKAP5 tethering of both PKA and AC8 to Cav 1.2 provides coordination of the complete positive feedback loop of calcium back onto $\mathrm{Ca}_{\mathrm{V}} 1.2$. In agreement with a previous model [103], our model shows that the positive stimulation of $\mathrm{AC} 8$ by $\mathrm{Ca}^{2+}$ drives in-phase PKA oscillations but our model extends this finding to identify that the AC8-stimulated PKA activity is necessary for the positive feedback needed to develop $\mathrm{Ca}^{2+}$ oscillations. Indeed, recent work by Dou et al. provides experimental evidence that knockdown of AC8 in the pancreatic $\beta$-cell line Ins-1 ablates the PKA activation in response to depolarization by $\mathrm{KCl}$ [125]. Interestingly, they observed this loss of $\mathrm{Ca}^{2+}$ stimulated PKA with a cytosolic 
PKA reporter even though the $\mathrm{Ca}^{2+}$ response to $\mathrm{KCl}$ was unchanged by the $\mathrm{AC} 8$ knockdown. Our AC8/AC5 model would predict that the AKAP5 PKA response would be lost, but that the plasma membrane PKA activity would be unaffected. In the future, experimental validation of this model prediction could be tested through the use of our AKAP5-AKAR4 and Lyn-AKAR4 biosensors combined with knockdown of AC8. Furthermore, mutation of our AKAP5-AKAR4 biosensor in AKAP5 residues that mediate AC8 localization [118] could be utilized to experimentally test if AC8 tethering specifically by AKAP5 is the driver of in-phase PKA oscillations and necessary for the development of $\mathrm{Ca}^{2+}$ oscillations. This non-canonical activation of PKA by $\mathrm{Ca}^{2+}$ stimulation of AC8 establishes crosstalk between $\mathrm{Ca}^{2+}$ and PKA signaling, and is central to the ability of AKAP5 to coordinate in-phase oscillations.

While PDE1 has been shown to be a critical component in the development of out-ofphase PKA and $\mathrm{Ca}^{2+}$ oscillations [21,103], we have identified a novel mechanism wherein the PKA activity at the plasma membrane can both oscillate out-of-phase with $\mathrm{Ca}^{2+}$ and increase from baseline after TEA stimulation. Gaining insight into the basal PKA activity is critical for two reasons: evaluating the positive feedback by PKA onto the $\mathrm{Ca}_{\mathrm{V}} 1.2$ channel and understanding the activation state of PKA regulated transcription factors. Contrastingly, the extended AC8/AC5 model suggested that positive feedback by the out-of-phase PKA activity was insufficient to generate regular $\mathrm{Ca}^{2+}$ oscillations in response to TEA stimulation (Figure 5.8B), but the previous model by $\mathrm{Ni}$ et al. [21], which only had out-of-phase PKA activity, was able to exhibit the $\mathrm{Ca}^{2+}$ oscillations. This discrepancy between these two models occurs due to the differences in the basal PKA activity prior to TEA stimulation. The Ni model only evaluated the $\mathrm{Ca}^{2+}$ dependent regulation of PDE1 which required a high basal AC activity (and therefore also a high basal PKA activity) to get out-of-phase PKA oscillations; consequently $\mathrm{Ca}_{\mathrm{V}} 1.2$ would be highly phosphorylated in the basal resting state 
and thus constantly primed for oscillations. On the other hand, Cav1.2 channels in the the AC8/AC5 model are primarily dephosphorylated at rest, and only the immediate and scaffold amplified positive feedback by the in-phase, AKAP5 tethered PKA is strong enough push the cell membrane potential past its depolarization threshold. Furthermore, this model provides a mechanistic interpretation of the importance of AKAP5 tethering to $\mathrm{Ca}_{\mathrm{V}} 1.2$ in the development of regular oscillations in response to TEA (Figure 5.1). Alternatively, a sustained high level of PKA activity in $\beta$-cells leads to translocation of the PKA catalytic subunit to the nucleus and which then activates the transcription factors CREB and PDX1 [126-128]. $\beta$-cells can experience sustained high PKA levels in stress conditions such as over-nutrition, and the activation of CREB and PDX-1 serve to compensate for this by promoting the increase in $\beta$-cell mass and insulin production $[127,129]$. Thus, maintaining a low basal PKA activity during normal physiologic function would be necessary to avoid $\beta$-cell hypertrophy and the over-production of insulin as well as allowing the cell to be able to respond to stress. Moreover, the oscillatory PKA activity in $\beta$-cells may have evolved to allow the cell to strongly activate immediate targets of PKA phosphorylation but avoid the translocation of PKA to the nucleus [126].

Previous work has hypothesized that PKC can mediate crosstalk between $\mathrm{Ca}^{2+}$ and PKA [130,131]. Our computational model suggests that AKAP5 can regulate the crosstalk between PKC and AC5, leading to the delayed and $\mathrm{Ca}^{2+}$-dependent activation of PKA at the plasma membrane. Experimental validation of this novel mechanism could be done by inhibiting PKC or knocking down AC5 expression and testing if plasma membrane PKA activity is ablated but the AKAP5 tethered PKA response is still maintained in either TEA induced oscillations or membrane depolarization by $\mathrm{KCl}$. If subsequent experimental validation shows that $\mathrm{PKC}$ is not responsible for the activation of plasma membrane PKA activity, our model still identified that there must be a delayed and $\mathrm{Ca}^{2+}$ dependent activation 
of adenylyl cyclase at the plasma membrane. This coordinated and distinct regulation of two different pools of adenylyl cyclase within the cell suggests that these two phases of PKA activation may both play an important functional role in signal transduction.

While there are several examples of in-phase or out-of-phase oscillations within cells or across groups of cells [132-135], the work shown here is, to the best of our knowledge, novel evidence of a single signaling component oscillating simultaneously in- and out-ofphase within two separate compartments of a cell. The ability of AKAP5 to coordinate simultaneous in- and out-of-phase PKA oscillations may allow the cell to reuse the same signaling pathway for the regulation of different processes $[23,136]$. Our work here showed that the in-phase PKA oscillations are necessary for the re-enforcement of calcium flux and thus provides the integral role of positive feedback in the development of oscillations [137]. For the out-of-phase PKA activity, the conservation of this complex mechanism suggests that this out-of-phase PKA activity plays a critical role in $\beta$-cell signaling. It is possible that the out-of-phase cAMP and PKA oscillations are necessary for either the insulin vesicle filling $[125,138]$ or promoting vesicle mobilization for exocytosis [139-141] which are important processes during the periods between calcium transients. In addition, this dual purpose encoding [142] of PKA signals by AKAP5 may provide the groundwork to develop synthetic scaffolds that can multiplex two responses for a single stimulus $[143,144]$. This evidence of polarized separation of compartmentalized PKA signals suggests that scaffold proteins in other cells could promote similar multi-purpose use of PKA or other signaling pathways.

In summary, this study provides experimental evidence and computational analysis of the coordination of both in- and out-of-phase PKA and $\mathrm{Ca}^{2+}$ oscillations by AKAP5. Our findings highlight the importance of scaffold proteins in the organization of unique signalosomes and diversifying the possible responses from a signaling enzyme. The significance of AKAP5 in this signaling mechanism demonstrates that targeting scaffold protein interac- 
tions may represent a novel approach to therapeutically disrupt crosstalk between specific signaling pathways. The importance of $\mathrm{Ca}^{2+}$ and PKA signaling in other cell types further emphasizes the importance of this AKAP5 coordinated $\mathrm{Ca}^{2+}$-PKA circuit across biology. 


\section{Chapter 6}

\section{Dissertation discussion}




\subsection{Global Aims}

The overall goal of this dissertation was to investigate the effects of scaffold proteins on cellular signaling and their underlying mechanisms. We hypothesized that scaffold proteins affect signal transduction for both individual reactions and coordinated crosstalk by tethering specific signaling networks and promoting increased enzyme-substrate interaction. In testing this hypothesis we have:

1. Proposed and validated the scaffold state-switching model as a novel mechanism for scaffold tethered enzymatic reactions, thus providing a mechanistic understanding of the amplification and acceleration seen on scaffolds.

2. Discovered that the scaffold state-switching mechanism leads to insulation of scaffoldtethered enzymes from inhibition by substrate- or ATP-competitive inhibitors but not activation-competitive inhibitors.

3. Demonstrated the ability of a scaffold protein to promote the development of complex signaling dynamics through the coordination of crosstalk between signaling pathways.

\subsubsection{Dynamics of AKAP7 $\alpha$ and AKAP5 tethered signal trans- duction}

In this dissertation, we examined these properties of scaffold proteins by studying the effects of AKAP7 $\alpha$ and AKAP5 on signal transduction.

In Chapter 4 (Aim 1) we described the development of the scaffold state-switching computational model and experimental validation with PKC and the scaffold protein AKAP7 $\alpha$. This model laid the fundamental groundwork to study the effects of scaffold tethering on signaling kinetics and quantitatively evaluate amplification or acceleration observed from 
scaffold tethering. Through this model we estimated that tethering of PKC by AKAP7 $\alpha$ results in a greater than 5 fold increase in the rate of enzyme-substrate interaction and this increase is the mechanism driving the amplification and acceleration. Extension of this model to study the effect of scaffold state-switching on different types of inhibitors led to the prediction that scaffold proteins can insulate tethered kinases from substrate- and ATP-competitive inhibitors but not activation-competitive inhibitors. Indeed, insulation of PKC from substrate- and ATP-competitive by AKAP7 $\alpha$ validated this novel model prediction. Collectively, these data support the scaffold state-switching model as a mechanism underlying scaffold-tethered enzymatic reactions.

In Chapter 5 (Aim 2) we described the application of computational models to evaluate the role of AKAP5 in the coordination of oscillatory PKA and $\mathrm{Ca}^{2+}$ signaling dynamics in MIN6 cells. These computational models were utilized to evaluate several hypotheses for mechanisms that could lead to our experimental observation that PKA oscillations are in-phase with $\mathrm{Ca}^{2+}$ on AKAP5 but out-of-phase at the plasma membrane. These models predicted that AKAP5 is critical for regulating the activation of two distinct pools of adenylyl cyclases, AC8 and AC5, that drive PKA oscillations through different $\mathrm{Ca}^{2+}$-dependent mechanisms of activation. This model was extended to incorporate the positive feedback by PKA on the Cav 1.2 calcium channel and suggested that the in-phase, AKAP5-tethered PKA activity was necessary for the initiation of $\mathrm{Ca}^{2+}$ oscillations in MIN6 cells. Together, these model predictions provide an example of the integral nature of scaffold proteins in the coordination of signaling proteins into specific signalosomes.

AKAP7 $\alpha$ and AKAP5 served as prototype scaffold proteins by which we could evaluate how scaffolds can create acceleration, amplification, insulation and specificity for tethered enzymatic reactions. Validation of the scaffold state-switching model with AKAP7 $\alpha$ was possible because it acts as a simple tether that does not directly affect PKC activity and 
does not bind negative regulators of PKC [86]. Conversely, AKAP5 binds several interacting signaling proteins creating a complex signalosome capable of coordinating crosstalk between different signaling pathways. Through AKAP7 $\alpha$ and AKAP5 we were able to test our theoretical understanding of the effects of scaffold proteins with the goal to apply this insight broadly to all scaffold proteins.

\subsubsection{Relevance to other scaffold proteins}

Our broad definition that scaffold proteins bind two or more signaling proteins encompasses a large number of proteins [145], but in this dissertation we sought to specifically study the effects of tethering on signal transduction. To study these effects, AKAPs have often served as prototypical scaffold proteins because many of the canonical AKAPs were originally identified to primarily act as simple tethers with little or no active participation in signal transduction [47]. Indeed, AKAPs besides AKAP7 $\alpha$, such as AKAP5, have been shown to amplify or accelerate signal transduction while acting as a simple tether [28,29,33]. Moreover, our work examining the coordination of signal transduction by AKAP5 is complimented by studies of different AKAP knockout mice (AKAP1 - [19] AKAP5 - [146, 147] AKAP9 [148]), that demonstrated the importance of scaffold proteins in the coordination of multiple signaling pathways. But the AKAP family of scaffold proteins are diverse and share little sequence similarity apart from the PKA binding domain that is believed to be a product of convergent evolution [149]. The combination of this independence between AKAPs and the occurrence of amplification and acceleration in different AKAPs suggests that these phenomena arise as a feature of scaffold tethering in general. Additionally, the concordance

of the scaffold state-switching model with experimental data did not require AKAP specific assumptions that would limit its applicability to all scaffold proteins. These data suggest 
that our insights into the effects of scaffold proteins may extend beyond the AKAP family of scaffold proteins.

In addition to AKAPs, there are other families of scaffold proteins that are classified by their ability to bind specific proteins, such as MAP Kinases [150,151], or the presence of protein binding domains, like SH3 [152] or PDZ domains [153]. In the MAPK scaffolds alone, several examples of amplification, acceleration and coordination have been identified. For example, the MAPK scaffold Arrestin-3 has been shown to amplify and accelerate JNK3 phosphorylation [154]. On the other hand, the MAPK scaffold Pax has been observed to exhibit very little amplification of MAPK signaling [53]. When we consider this in the context of our sensitivity analysis on the scaffold state-switching model (Figure 4.8), these data may indicate that the kinetics of the MAPKs associated with Pax may exist in a kinetic domain where the scaffold does not exhibit much amplification but may significantly accelerate the rate of signal transduction. Interestingly, our sensitivity analysis would also suggest that this Pax scaffold may strongly insulate the tethered MAPKs from substrate-competitive inhibitors, but this has not been tested experimentally. Additionally, the MAPK scaffolds Ste 5 and Pbs2 are great examples of scaffold proteins that coordinate specific signaling pathways to create a distinct signaling response [83], similar to the coordination of in-phase PKA activity on AKAP5 in MIN6 cells (Chapter 5). The breadth of scaffold proteins that amplify, accelerate and coordinate signal transduction serves to further strengthen our hypothesis that these effects are fundamental properties of scaffold tethering.

While several of the scaffold proteins discussed previously are considered to act as simple scaffolds, there are also proteins that can both act as a scaffold and actively regulate signal transduction. To better understand the impacts of both the active and passive contributions of these scaffold proteins, the scaffold state-switching model can be extended to incorporate the effects of scaffold proteins that extend beyond the simple tethering of signaling proteins. 
For example, some scaffold proteins can directly regulate the intrinsic activity of a tethered enzyme. This can either increase activity, such as the allosteric activation of the MAPK Fus3 by binding to the Ste5 scaffold protein [155], or inhibit activity, as seen in the inhibition of PKC activity when bound to AKAP5 [26]. Furthermore, some regulatory subunits of signaling proteins can have an under-appreciated role as a scaffold that tethers the catalytic subunit to specific substrates. For example, regulatory subunits of protein phosphatases can bind to distinct protein complexes thus promoting substrate specificity of these promiscuous enzymes [156-160]. Evaluating these direct effects in the scaffold state-switching model could identify if the combination of the active and passive roles of these scaffold proteins are synergistic, antagonistic or purely additive.

Scaffold proteins can also play an active role in shaping cell signaling through the dynamic regulation of binding affinities of tethered signaling proteins. For example, the affinity of PKD for AKAP-Lbc is decreased when AKAP-Lbc is phosphorylated by PKA and this decrease in PKD affinity actually leads to an increase in PKD activation due to the increased substrate turnover [30]. While we did not examine the effects of scaffold association and dissociation in Chapter 4, because of the high binding affinity of PKC for AKAP7 $\alpha$, extending the model to allow enzyme and substrate dissociation would not affect the fundamental assumptions of the scaffold state-switching model. These examples of proteins that function as both scaffolds and active regulators of signal transduction highlight the diverse array of scaffold proteins and the importance of considering the effect tethering in multi-protein complexes.

In this dissertation, we examined the roles of proteins that are scaffolds and proteins that are tethered in signaling transduction but this work may extend beyond proteins to evaluate scaffolds that promote interactions between different types of cellular macromolecules. There is a family of "bridging proteins" that contain co-regulators of transcription which 
tether transcription factors to transcriptional machinery or other regulators of transcription [161]. For example, CREB-binding protein (CBP) was originally identified to bind the cAMP-response element binding protein (CREB) transcription factor but has since been shown to bind several different transcription factors [161]. In addition to binding transcription factors, CBP can tether these transcription factors to transcriptional elements or histone acetyltransferases to coordinate the regulation of gene transcription [162]. While $\mathrm{CBP}$ is promoting the interaction between proteins and DNA (instead of protein to protein), the scaffold state-switching model could be extended to this system, suggesting that these bridging proteins may accelerate and amplify the regulation of gene transcription. One noteworthy difference is that when the CBP scaffolds the transcriptional machinery, the transcription complex must leave the scaffold in order to perform its function. Although this may negate the amplification by scaffold proteins, it may greatly accelerate the initiation of the first transcription. Conversely, scaffolds need not be limited to proteins, as other cellular macromolecules can also specifically bind several signaling molecules. For example, the long intergenic non-coding RNA NRON (non-coding repressor of NFAT) can tether the Nuclear factor of activated T cells (NFAT) and IQ motif containing GTPase activating protein (IQGAP) proteins to promote the phosphorylation of NFAT and suppress its role as a transcription factor [163].

Additionally, scaffold proteins may also play a role outside of cellular signaling. For example, the CipA protein in Clostridium acetobutylicum tethers cellulose to the multiple enzymes required to degrade cellulose, thus forming a "cellulosome", in order to hydrolyze this notoriously resistant substrate $[164,165]$. Again, these cellulosome scaffold proteins (also referred to as "Scaffoldin" proteins in metabolism literature) can amplify and accelerate the metabolism of cellulose similar to that seen with the scaffold state-switching model [166] and may promote substrate channeling between sequential enzymes [167]. These examples 
indicate the foundational role that scaffold tethering can play across cellular functions.

In addition to scaffold proteins, cells can organize and compartmentalize signal transduction by several different means and the insights into the effects of scaffold proteins described in this dissertation may be applied to these as well. For instance, lipid rafts can concentrate certain membrane targeted and integral membrane proteins thus promoting their interaction [168]. However, the mechanism by which the enzyme-substrate interaction would be increased by these lipid rafts is fundamentally different than the assumptions made in the scaffold state-switching model. The key distinction being that our scaffold state-switching model assumes that the scaffold condenses the two signaling proteins into a solid-state device (discrete states), whereas lipid rafts serve to increase the density of the proteins. Interestingly, interactions between proteins in the plasma membrane can occur 1-2 orders of magnitude faster than if they were to occur in solution due to the reduced dimensionality of a 2-dimensional surface but this advantage may be lost if the protein must first translocate and associate with the plasma membrane [169]. Conceptually one could consider scaffolds as changing the dimension from either $3 \mathrm{D}$ or $2 \mathrm{D}$ to " $0 \mathrm{D}$ " (only 2 states), but the more technically correct difference is that the scaffold state-switching model changes the enzyme-substrate association reaction from a second order to a first order reaction. While other mechanisms of compartmentalization can also create similar effects as scaffold proteins, the solid-state nature of the scaffold state-switching model distinguishes the unique function of scaffold proteins in cell signaling.

In this dissertation, we identified and validated that scaffold proteins can create acceleration, amplification, insulation and specificity for enzymatic reactions by tethering specific signaling proteins and promoting their interactions. Indeed, the effects scaffold tethering on a diverse set of signaling proteins indicate the importance of scaffold proteins in numerous cellular functions. In conclusion, the fundamental properties of scaffold proteins make them 
integral for the efficient and coordinated signal transduction necessary for proper cellular communication.

\subsection{Future directions}

The fundamental nature of this work opens up several interesting opportunities for future work studying the impacts of scaffold tethering on both individual reactions and across the signaling network. Specifically, our work motivates follow-up studies in the following areas:

- Extension to other signaling proteins and scaffolds

- Evaluation of tethered signaling motifs

- (Re)defining the structure of signaling networks

- Identification of therapeutic targets and synthetic scaffold design

\subsubsection{Additional signaling proteins and scaffolds}

The minimal assumptions underlying the scaffold state-switching mechanism allow this model to be applied to the numerous enzymatic reactions tethered to a scaffold. Here we validated this mechanism using $\mathrm{PKC}$ and $\mathrm{AKAP} 7 \alpha$, and further validation for other signaling proteins would strengthen the evidence supporting the general applicability of the scaffold state-switching model. Specifically, the prototypical kinase PKA would be of particular interest because of its importance in cell signaling and the $>70$ different AKAPs that scaffold PKA $[95,96,170]$. Mirroring our work done in Chapter 4, scaffold state-switching models of PKA activity could be coupled to experimental validation with the simple scaffold AKAP7 $\alpha$ 
and the PKA biosensor AKAR4 [108]. This work could both demonstrate the general applicability of the scaffold state-switching mechanism and provide insight into some of the factors that affect the scaffold efficiency number, $\epsilon$. Further into the future, after several enzyme-scaffold complexes have been evaluated with the scaffold state-switching model, it may become possible to identify what biophysical properties of the scaffold or enzyme affect the rate of enzyme-substrate interaction on the scaffold. Indeed, Smith et al. have shown that the adjusting length of the linker region of the regulatory subunit of PKA, which itself acts as a scaffold for the catalytic subunit, can alter the enzyme kinetics [171]. Interestingly, they found that a shorter linker accelerated and amplified the response to stimulation suggesting that enzymes and substrates that are tethered nearer to each other on the scaffold may exhibit a larger scaffold efficiency number [171]. Understanding the biophysical properties that define the $\epsilon$ for a given enzyme-substrate-scaffold complex will provide us with a better understanding of the biology and enable the prediction of the $\epsilon$ for untested com-

plexes. Furthermore, this study highlights the ability of regulatory subunits to both directly modulate enzyme activity and serve as a scaffold to promote substrate specificity [156-160]. Evaluation of the scaffold state-switching model on other signaling proteins and scaffolds will either serve to strengthen its general applicability or provide additional data with which to refine the model of tethered signal transduction.

\subsubsection{Scaffold-tethered signaling motifs}

In Chapter 4 we studied the effects of scaffold tethering on a single enzyme and substrate pair, but many of the identified scaffold proteins have been shown to bind several interacting signaling proteins at a time $[149,172]$. The combination of multiple signaling proteins on a single scaffold can lead to the coordination of signaling motifs that are sufficient to exhibit 
adaptive, oscillatory and ultrasensitive signaling dynamics [38, 40,44]. For example, our work in Chapter 5 showed that AKAP 5 organizes a positive feedback loop that is critical for the development of oscillatory $\mathrm{Ca}^{2+}$ dynamics. Analysis of tethered signaling motifs with the scaffold state-switching mechanism would provide insight into the quantitative effects of scaffold tethering on the signaling dynamics. This computational modeling would be important because these nonlinear dynamics exist with a subset of kinetic regimes and it will be important to understand if scaffold tethering can push signaling into or out of a required signaling regime $[173,174]$. For example, negative feedback can lead to either an adaptive signaling response or a simple monotonic increase depending on the strength and time delay of the negative feedback [38], which are both kinetic parameters that can be affected by scaffold tethering.

One such example tethered signaling motif, is the cAMP-PKA-PDE4 negative feedback loop that is coordinated by the scaffold proteins AKAP7 $\delta, \operatorname{mAKAP}$ and Yotiao $[175,176]$. In this feedback loop, PKA phosphorylation of PDE4 increases the rate of degradation of cAMP by PDE4 [177]. Dodge-Kafka et al. developed a synthetic scaffold that tethers both PKA and PDE4 and showed that this scaffold complex does indeed exhibit an adaptive response [24]. In addition, recent evidence has shown that PDEs may directly promote the dissociation of cAMP from the regulatory subunit of PKA thus directly terminating PKA activity $[178,179]$. Therefore, a scaffold state-switching model of this signaling complex could determine if the adaptation arises from the scaffold promoting PKA phosphorylation of PDE, PDE direct inactivation of PKA, or both, and how sensitive this system is to scaffold dependent changes. Extension of this analysis to the endogenous scaffolds that bind both PDE and PKA (e.g. mAKAP or AKAP7 $\delta$ ) would also test the sensitivity of this feedback loop to scaffold differences and allow the study of the downstream effects of this adaptive response. By assembling these signaling motifs, scaffold proteins can act as "solid-state" 
signaling devices [91] to process and transform signaling inputs.

\subsection{3 (Re)structure of signaling networks}

To evaluate the role of scaffold proteins on physiological and pathological outcomes we must begin to examine how scaffold proteins shape whole signaling networks. In Chapter 5, we showed the essential role of AKAP5 in the development of the oscillatory $\mathrm{Ca}^{2+}$ dynamics which are required for the pulsatile secretion of insulin in $\beta$-cells [97]. But the process of insulin secretion requires the coordination of additional signaling machinery [180] and indeed the scaffold proteins RIM $[140,181]$ and AKAP7 [182] have been shown to also regulate the secretion of insulin. The extent of signaling network coordination by different scaffold proteins is even more apparent in cardiac PKA signaling where at least 14 AKAPs have been identified $[172,183,184]$. In Figure 6.1, we can see that most of species in the $\beta$-adrenergic signaling pathway are tethered by different AKAPs [110]. While there have been systems level studies of signal transduction through these pathways, providing insight into the role of compartmentation $[45,78,110]$, incorporation of AKAPs into these models could reveal the underlying organization of the whole network. Even without knowledge of the kinetic effect of scaffolding for each tethered reaction, tools such as normalized hill modeling [22] could be used to gain insight into the effect of different scaffolds on network topology. Additionally, incorporation of scaffold proteins into these normalized hill models could indirectly create compartmentalized signaling, possibly even unknown compartment organization, not currently in the model. The complexity of these scaffold-organized signaling networks suggest that taking a system-level view of this scaffold "interactome" will be fundamental to understanding and altering signal transduction. 


\subsubsection{Drug targets and synthetic scaffolds}

The promotion of signaling enzyme and substrate interaction by scaffold proteins can lead to context dependent specificity for promiscuous signaling proteins [185]. This has engendered the hypothesis that disruption of scaffold tethering presents a novel drug target to inhibit specific signaling outcomes [186]. By providing a quantitative framework by which to study scaffold tethered signaling, the work presented in this dissertation will enable the analysis and guidance toward this hypothesis. Already, the scaffold state-switching model has predicted that scaffold proteins can insulate tethered enzymes from substrate- and ATP-competitive inhibitors (Chapter 4). On the other hand, drugs targeting scaffold proteins interactions present a fundamentally different method of inhibition. Where traditional inhibitors block the catalytic activity, scaffold targeted drugs could reduce the signaling received by the substrates that are tethered specifically to that scaffold. In the future, scaffold protein models could simulate the kinetic effects of disrupting the tethering different signaling proteins from a signaling complex. Moreover, the development of scaffold network models could be used to predict the functional outcome of disrupting specific scaffold interactions and identify the most salient interactions to disrupt. With the recent development of AKAP isoform-specific PKA disruption peptides [187], this type of model could identify the best candidates for targeting diseases like cardiac hypertrophy [188]. These advances present the possibility of being able to develop drugs for specific targets of signaling proteins that would otherwise be "un-druggable" because of the other detrimental side effects that would occur.

Additionally, the coordination of signal transduction by scaffold proteins through combination of different, and possibly modular, protein binding domains has inspired the biomimetic use of scaffold proteins in synthetic biology [93]. Application of the scaffold state-switching model to study synthetic scaffolds could both guide their design and also provide insight 
into the underlying design of endogenous scaffolds. Experimentally taking protein binding domains and linking them together with different synthetic linkers, similarly to the work done by Smith et al. on the PKA regulatory domain, would allow us to probe the design principles of the scaffold efficiency, $\epsilon$ [171]. Additionally, the tunable and modular nature of these synthetic scaffolds would allow the testing of the effects of tethering different signaling motifs onto a scaffold [189-191]. On a larger scale, network scale models of scaffold coordinated signal transduction could be used also evaluate the outcomes and design of network "re-wiring" through the use of synthetic scaffolds [83,192]. As seen by the unique dynamics coordinated by AKAP5, the work in dissertation provides tools that are needed to design, optimize and utilize synthetic scaffolds for biomanufacturing and therapeutic translation.

\subsection{Conclusions}

Since the identification of protein binding domains in the late 1980s, such as the SH2 domain [193] or PKA binding proteins [194,195], the number of scaffold and adaptor linked signaling proteins has increased tremendously [93]. Additionally, recent proteomics work identifying 1,278 human proteins found in multi-protein complexes suggests that the organization of proteins by scaffolds may be more ubiquitous than previously thought [145]. The combination of this large number of possible scaffolded proteins and the promiscuity of signaling enzymes [185] suggests that scaffold tethered signal transduction could be more of the rule, rather than the exception. Thus, this work could provide a keystone in the foundation of a shift in the current paradigm of cellular enzyme kinetics [87]. In conclusion, by elucidating fundamental mechanisms that underlie the effects of scaffold proteins, our work expands our understanding cellular communication and provides tools to evaluate scaffold interactions. 


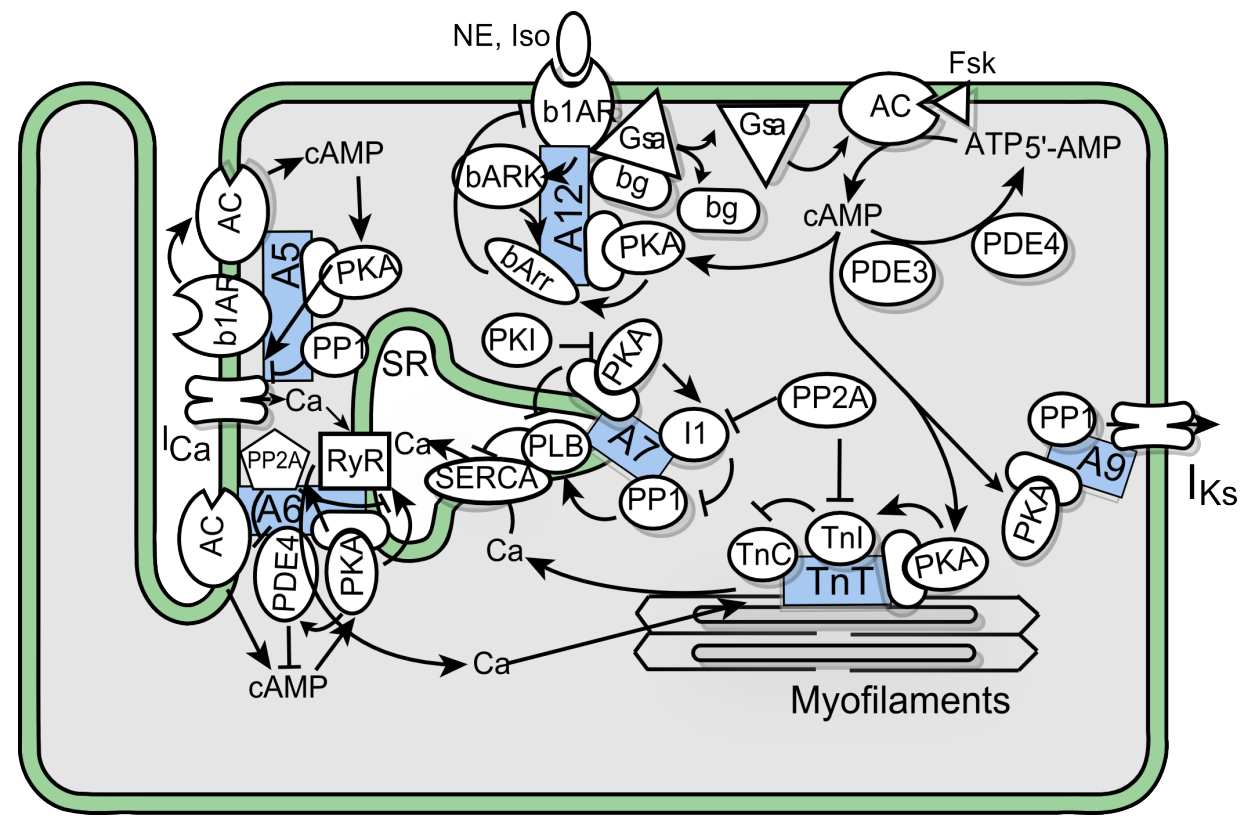

Figure 6.1: Scaffold proteins coordinate most of the key signaling proteins in the cardiac $\beta$ adrenergic signaling pathway. Figure adapted from Yang and Saucerman JMCC 2012 [110] 


\section{Appendix A}

\section{Scaffold state-switching model}

\section{description}

Reprinted from supplemental materials of: E.C. Greenwald, J.M. Redden, K.L. Dodge-Kafka, J.J. Saucerman. "Scaffold State-Switching Amplifies, Accelerates and Insulates PKC Signaling." Journal of Biological Chemistry Vol. 289 pp 2353-2360 (2014), with permission from the American Society for Biochemistry and Molecular Biology 


\section{A.1 Model Description}

Here we will describe the model assumptions and equations. The model is an Ordinary Differential Equation, ODE, model that was solved using MATLAB's built in ODE solver ode15s.

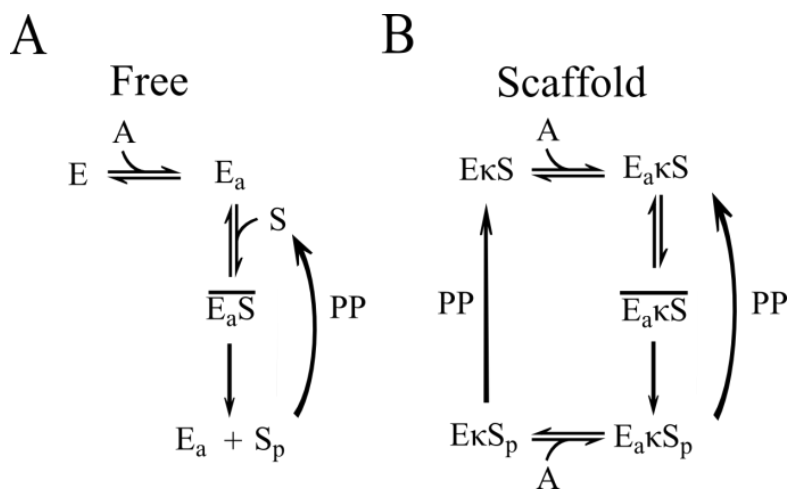

Figure A.1: Model reaction diagram. A) The free model represents enzyme, $E$, catalysis of phosphorylating the substrate, $S$, to by first forming the active intermediate, $\overline{E_{a}} S$, and then form the product, $S_{p}$, in solution. B) The scaffold model considers the enzyme-scaffold-substrate complex, $E \kappa S$, as a whole. This model incorporates scaffold state-switching when the enzyme is active the complex can stochastically switch between inactive, $E_{a} \kappa S$, and active, $\overline{E_{a} \kappa S}$, intermediates before completing the catalysis to the phosphorylated product, $E_{a} \kappa S_{p}$. In both models the phosphorylation is reversed by phosphatases, $P P$.

Table A.1: Definition of model species

\begin{tabular}{|cl|}
\hline Symbol & Definition \\
\hline$E$ & Enzyme $(P K C)$ \\
$S$ & Substrate $(C K A R)$ \\
$E_{a}$ & Activated enzyme $\left(P K C_{a}\right)$ \\
$S_{p}$ & Phosporylated enzyme $\left(C K A R_{p}\right)$ \\
$A$ & Enzyme agonist (PDBu) \\
$E_{a} S$ & Enzyme-substrate active intermediate \\
$\kappa$ & Scaffold complex \\
\hline
\end{tabular}




\section{A.1.1 Free Model}

The "Free Model" is a model of PKC phosphorylation of CKAR in solution using the standard enzyme mechanism. The model will be described in general where PKC is the enzyme, $E$, and CKAR is the substrate, $S$. First we assume a closed system so the total concentration of PKC and CKAR remain constant.

$$
\begin{aligned}
& E_{t o t}=E+E_{a}+\overline{E_{a} S} \\
& S_{t o t}=S+\overline{E_{a} S}+S_{p}
\end{aligned}
$$

\section{PKC activation}

Here we assume that the enzyme can be activated by binding of either a small molecule agonist, A, or basal amounts of endogenous enzyme activators. The basal activity of the enzyme was included because PKC has been shown to have a significant amount of basal activity with CKAR [75]. Upon dissociation of the activator from the enzyme, the enzyme is no longer active.

$$
R_{1}=\left(k_{a a} \cdot A+k_{a b}\right) \cdot E-k_{a d} \cdot E_{a}
$$

\section{CKAR phosphorylation}

The enzyme and substrate must first associate to form the active enzyme-substrate intermediate, then the enzyme can catalyze the phosphorylation of the substrate. The rate of net enzyme-substrate association can be written as follows:

$$
R_{2}=k_{e s a} \cdot S \cdot E_{a}-k_{e s d} \cdot \overline{E_{a} S}
$$


The rate of catalysis can then be written as,

$$
R_{3}=k_{\text {cat }} \cdot \overline{E_{a} S}
$$

\section{CKAR phosphorylation}

Here we have modeled the substrate dephosporylation as a simple first order irreversible reaction.

$$
R_{4}=k_{\text {ppase }} \cdot S_{p}
$$

Now the ODE's can be formed as follows,

$$
\begin{gathered}
\frac{d S_{p}}{d t}=R_{3}-R_{4} \\
\frac{d \overline{E_{a} S}}{d t}=R_{2}-R_{3} \\
\frac{d E_{a}}{d t}=R_{1}-R_{2}+R_{3}
\end{gathered}
$$

where the $E$ and $S$ are determined using the mass balance equations (eqns. A.1 and A.2, respectively).

\section{A.1.2 Scaffold State-Switching Model}

The scaffold state-switching model, referred here as the scaffold model, is our hypothesis for how reactions on scaffold occur. Most of the reactions are very similar except for the enzymesubstrate association, the novel aspect of the scaffold state-switching model. The reaction rates are the same for both models with the exception of, $k_{\epsilon}$, the rate of state switching for the enzyme-scaffold-substrate complex to the active intermediate. Here we assume that 
effects from enzyme and substrate dissociation are negligible because the substrate in our experimental example (CKAR) is permanently tethered to the scaffold and because PKC has been shown to have a high affinity for AKAP7 $\alpha$ [86]. Therefore, we must consider the state of both the enzyme and substrate for each state of the complex as a whole. Due to this assumption the mass balance equation becomes the following,

$$
E \kappa S_{t o t}=E \kappa S+E_{a} \kappa S+\overline{E_{a} \kappa S}+E_{a} \kappa S_{p}+E \kappa S_{p}
$$

where $\kappa$ denotes the scaffold $(\mathrm{AKAP} 7 \alpha)$.

\section{Enzyme Activation}

$$
R_{1 S}=\left(k_{a a} \cdot A+k_{a b}\right) \cdot E \kappa S-k_{a d} \cdot E_{a} \kappa S
$$

Because of fixed stoichiometry we must now also consider activation of the enzyme that is complexed with phosphorylated substrate.

$$
R_{5 S}=\left(k_{a a} \cdot A+k_{a b}\right) \cdot E \kappa S_{p}-k_{a d} \cdot E_{a} \kappa S_{p}
$$

\section{CKAR phosphorylation}

The main difference proposed here is the enzyme and substrate can stochastically transition between inactive and active intermediate states, which occur through first order state transition reactions.

$$
R_{2 S}=k_{\epsilon} \cdot E_{a} \kappa S-k_{\text {esd }} \cdot \overline{E_{a} \kappa S}
$$


Again the catalytic rate constant and reaction order is not changed.

$$
R_{3 S}=k_{c a t} \cdot \overline{E_{a} \kappa S}
$$

\section{CKAR dephosporylation}

The phosphatase activity is assumed to not be affected by the substrate tethering to the scaffold.

$$
R_{4 S}=k_{\text {ppase }} \cdot E_{a} \kappa S_{p}
$$

Because of the fixed stoichiometry, the dephosporylation of the substrate that is tethered to an inactive enzyme must also be accounted for.

$$
R_{6 S}=k_{\text {ppase }} \cdot E \kappa S_{p}
$$

\section{ODE Reactions}

Now the scaffold ODE equations can be written.

$$
\begin{gathered}
\frac{d E_{a} \kappa S_{p}}{d t}=R_{3 S}-R_{4 S}+R_{5 S} \\
\frac{d E \kappa S_{p}}{d t}=-R_{5 S}-R_{6 S} \\
\frac{d \overline{E_{a} \kappa S}}{d t}=R_{2 S}-R_{3 S} \\
\frac{d E_{a} \kappa S}{d t}=R_{1 S}-R_{2 S}+R_{4 S}
\end{gathered}
$$

Parameter values for the above equations were determined from literature sources (Table A.2) or given a best initial guess (Table A.3). For the free model, the total substrate 
concentration, $[S]_{\text {tot }}$, was assumed to be equal to the total enzyme concentration. When the fraction of CKAR phosphorylated all states where CKAR is phosphorylated were included.

Table A.2: Constant model parameters.

\begin{tabular}{|lllcl|}
\hline Symbol & Definition & Units & Value & Source \\
\hline$k_{\text {cat }}$ & PKC catalysis rate & $s^{-1}$ & 4.4 & {$[196]$} \\
$K_{m}(P K C)$ & PKC Michaelis-Menten Const. & $\mu M$ & 57 & {$[197]$} \\
$k_{\text {esd }}$ & Enzyme-substrate dissociation rate & $s^{-1}$ & 17.6 & assumed $4 k_{\text {cat }}$ \\
$k_{\text {esa }}$ & Enzyme-substrate association rate & $\mu M^{-1} s^{-1}$ & 0.3860 & Calc. from \\
& & & & $K_{m}(P K C)$ \\
$K_{d}(P D B u)$ & PDBu Dissociation constant & $\mu M$ & 0.00799 & {$[198]$} \\
$k_{a d}$ & PDBu dissociation rate & $s^{-1}$ & 0.013 & {$[198]$} \\
$k_{a a}$ & PDBu association rate & $\mu M^{-1} s^{-1}$ & 1.6229 & Calc. from $k_{a d}$ \\
& & & & and $K_{d}(P D B u)$ \\
\hline
\end{tabular}

Table A.3: Fitted model parameters. The initial value is the parameter value used to generate model predictions in Figure 4.2. The fitted value is the parameter value obtained by fitting to the experimental data in Figure 4.3. CI range indicates 95\% confidence interval.

\begin{tabular}{|lllcll|}
\hline Symbol & Definition & Units & Init. Value & Fitted Val. & CI Range \\
\hline$k_{\text {ppase }}$ & $\begin{array}{l}\text { Phosphatase dephosph. } \\
\text { rate }\end{array}$ & $s^{-1}$ & $5.5 \cdot 10^{-3}$ & $4.79 \cdot 10^{-3}$ & $\pm 2.2 \cdot 10^{-3}$ \\
$k_{a b}$ & $\begin{array}{l}\text { Basal enzyme activation } \\
\text { rate }\end{array}$ & $s^{-1}$ & $1.08564 \cdot 10^{-4}$ & $4.579 \cdot 10^{-3}$ & $\pm 9.2 \cdot 10^{-3}$ \\
{$[E]_{t o t}$} & $\begin{array}{l}\text { Total enzyme conc. } \\
\epsilon\end{array}$ & $\mu M$ & 0.1 & 0.0397 & \pm 0.012 \\
& $\begin{array}{l}\text { Scaffold efficiency } \\
\text { number }\end{array}$ & - & 5 & 5.697 & \pm 0.37 \\
\hline
\end{tabular}

\section{A.1.3 Model Fitting}

To fit the model to the data, we normalized the model to the experimental data. To do this, we assumed the dynamic ranges of both sensors are the same since they contain the same CKAR biosensor. This is further supported by the similar responses of MyrCKAR and 
AKAP7 $\alpha$-CKAR to combined PKC activation and phosphatase inhibition (Figure 4.5). Thus we assume a constant scaling factor $(\phi)$ for converting from FRET ratio $(R)$ to fractional CKAR phosphorylation $(P)$ for both free and scaffold models. Thus the model is scaled then normalized to the basal activity state, similar to how the FRET data is normalized to 1 for the initial value (Eqn. A.21).

$$
R=\phi\left(P-P_{0}\right)+1
$$

Then to determine $\phi$ we used the steady-state FRET response $\left(R_{\text {Max }}\right)$ of MyrCKAR after $\mathrm{PDBu}$ stimulation and assumed this was directly related to the fractional CKAR phosphorylation in this condition $\left(P_{\operatorname{Max}}\right)$.

$$
R_{\text {Max }}=\phi\left(P_{\text {Max }}-P_{0}\right)+1
$$

Thus the scaling value is the ratio of the change in the FRET ratio to the change in CKAR phosphorylation.

$$
\phi=\frac{R_{\text {Max }}-1}{P_{\text {Max }}-P_{0}}
$$

Using this normalization scheme we used a built-in MATLAB non-linear least squares function (lsqcurvefit) to fit the model to the data in Figure 4.3. To confirm that the normalization did not introduce fitting artifacts, we also examined the predicted fraction of phosphorylated CKAR (Figure A.2B). The 95\% confidence intervals for fitted parameters were estimated from the coefficient covariance matrix (Table A.3). 
A

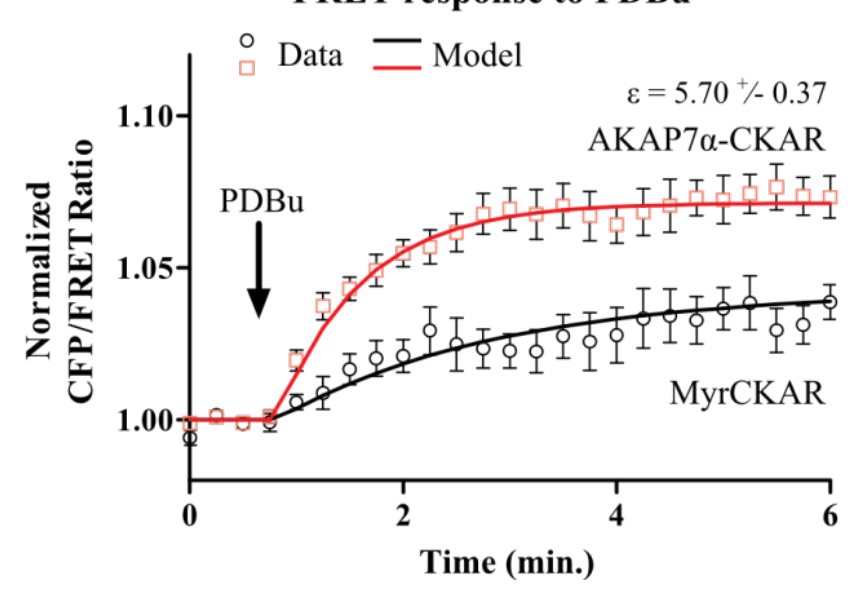

B

Model Fit Results

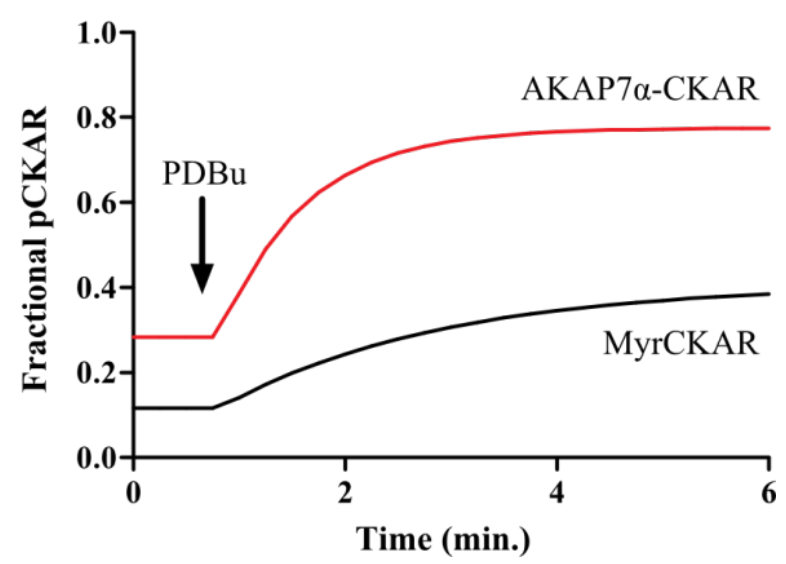

Figure A.2: Fitting of the model to the data. (A) Panel replicated from Figure 4.3B for clarity. (B) The model fit results shown as the fraction of phosphorylated CKAR.

\section{A.2 Model extension to include enzyme inhibitors}

The core model described above was extended to examine how the scaffold state-switching model affects the sensitivity to different classes of enzyme inhibitors (Figure A.3).

\section{A.2.1 Free Model}

\section{Activation Competitive Inhibitors}

The activation competitive inhibitor, $I_{a}$, is assumed to be able to bind only to the inactive enzyme.

$$
R_{5}=k_{i a a} \cdot I_{a} \cdot E-k_{i d a} \cdot E I_{a}
$$


A

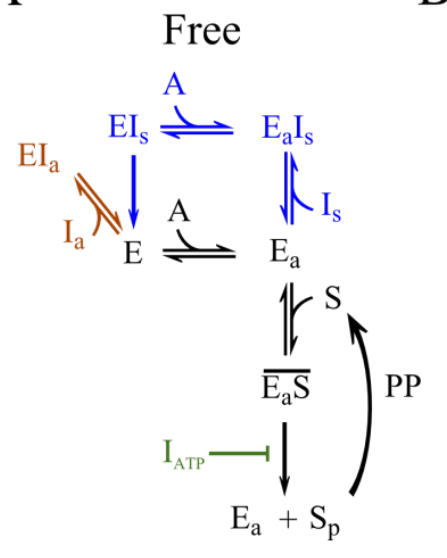

B

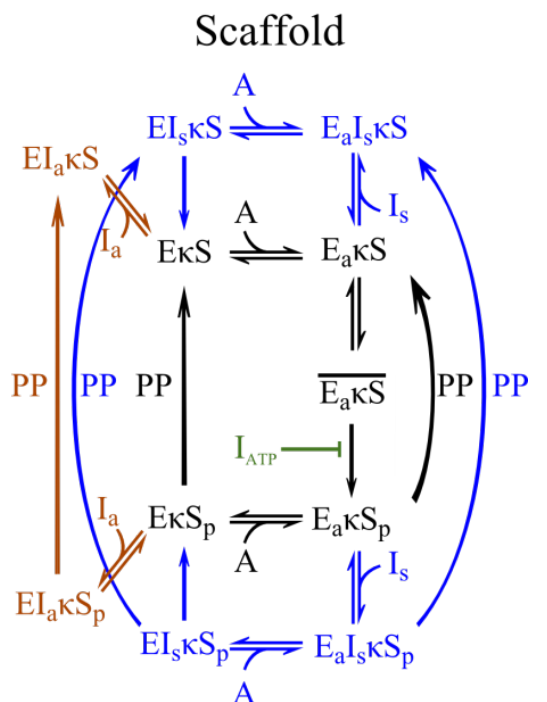

Figure A.3: Network diagram of the extended model with activation-competitive, substratecompetitive, and ATP-competitive inhibitors. The Free model represents the reaction occurring in solution and the Scaffold model is the reaction occurring on a scaffold. The original model (black) is the same as described earlier (Figure A.1). The activation-competitive inhibitor (brown) included the binding of the inhibitor, $I_{a}$, to the inactive enzyme, $E$, to form the inhibited enzyme, $E I_{a}$. The same process occurs in the scaffold except the inhibitor also can bind the complex with a phosphorylated substrate $\left(E \kappa S_{p}\right)$ and this substrate can be dephosporylated by PPase. Substratecompetitive inhibition (blue) involves the binding of the inhibitor, $I_{s}$, to the active enzyme, $E_{a}$, and the enzyme can dissociate and associate with the activator, $A$, while the inhibitor is bound. Again, the inhibition in the scaffold model mirrors that of the free model but also must account for the phosphorylation state of the substrate within the enzyme-scaffold-substrate complex. Finally, the ATP-competitive inhibitor (green), $I_{A T P}$, is shown as inhibiting the catalytic rate constant, $k_{\text {cat }}$. 


\section{ATP competitive Inhibitors}

The ATP competitive inhibitor was assumed to reduce $k_{\text {cat }}$ in a competitive manner against ATP binding to PKC.

$$
\hat{k}_{c a t}=k_{\text {cat }} \frac{A T P}{K_{m A T P}\left(1+I_{A T P} / K_{i A T P}\right)+A T P}
$$

\section{Substrate Competitive Inhibitors}

The substrate competitive inhibitor is assumed to be able to bind to the active enzyme when the enzyme is not in the enzyme-substrate active intermediate. It is also assumed that the activator can freely associate and dissociate with enzyme when the substrate-competitive inhibitor is bound.

$$
\begin{gathered}
R_{6}=k_{i a S} \cdot I_{S} \cdot E_{a}-k_{i d S} \cdot E_{a} I_{S} \\
R_{7}=\left(k_{a a} \cdot A+k_{a b}\right) \cdot E I_{S}-k_{a d} \cdot E_{a} I_{S} \\
R_{8}=k_{i d S} \cdot E I_{S}
\end{gathered}
$$

\section{ODEs}

$$
\begin{gathered}
\frac{d S_{p}}{d t}=R_{3}-R_{4} \\
\frac{d \overline{E_{a} S}}{d t}=R_{2}-R_{3} \\
\frac{d E_{a}}{d t}=R_{1}-R_{2}+R_{3}-R_{6} \\
\frac{d E I_{a}}{d t}=R_{5} \\
\frac{d E_{a} I_{S}}{d t}=R_{6}+R_{7}
\end{gathered}
$$




$$
\frac{d E I_{S}}{d t}=-R_{7}-R_{8}
$$

\section{A.2.2 Scaffold Model}

The scaffold model makes the same assumptions as the free models with respect to the inhibitor actions, but the scaffold model must account for both the enzyme's and substrate's activation and phosphorylation state, respectively.

\section{Activation Competitive Inhibitors}

$$
\begin{gathered}
R_{7 S}=k_{i a a} \cdot I_{a} \cdot E \kappa S-k_{i d a} \cdot E I_{a} \kappa S \\
R_{8 S}=k_{i a a} \cdot I_{a} \cdot E \kappa S_{p}-k_{i d a} \cdot E I_{a} \kappa S_{p} \\
R_{9 S}=k_{\text {ppase }} \cdot E I_{a} \kappa S_{p}
\end{gathered}
$$

\section{ATP competitive Inhibitors}

$$
\hat{k}_{c a t}=k_{\text {cat }} \frac{A T P}{K_{m A T P}\left(1+I_{A T P} / K_{i A T P}\right)+A T P}
$$

\section{Substrate Competitive Inhibitors}

$$
\begin{gathered}
R_{10 S}=k_{i a S} \cdot I_{S} \cdot E_{a} \kappa S-k_{i d S} \cdot E_{a} I_{S} \kappa S \\
R_{11 S}=\left(k_{a a} \cdot A+k_{a b}\right) \cdot E I_{S} \kappa S-k_{a d} \cdot E_{a} I_{S} \kappa S \\
R_{12 S}=k_{i d S} \cdot E I_{S} \kappa S
\end{gathered}
$$

We must also account for the phosphorylated substrates.

$$
R_{13 S}=k_{i a S} \cdot I_{S} \cdot E_{a} \kappa S_{p}-k_{i d S} \cdot E_{a} I_{S} \kappa S_{p}
$$




$$
\begin{gathered}
R_{14 S}=\left(k_{a a} \cdot A+k_{a b}\right) \cdot E I_{S} \kappa S_{p}-k_{a d} \cdot E_{a} I_{S} \kappa S_{p} \\
R_{15 S}=k_{i d S} \cdot E I_{S} \kappa S_{p} \\
R_{16 S}=k_{\text {ppase }} \cdot E_{a} I_{S} \kappa S_{p} \\
R_{17 S}=k_{\text {ppase }} \cdot E I_{S} \kappa S_{p}
\end{gathered}
$$

\section{ODEs}

$$
\begin{gathered}
\frac{d E_{a} \kappa S_{p}}{d t}=R_{3 S}-R_{4 S}+R_{5 S}-R_{13 S} \\
\frac{d E \kappa S_{p}}{d t}=-R_{5 S}-R_{6 S}-R_{8 S}+R_{15 S} \\
\frac{d \overline{E_{a} \kappa S}}{d t}=R_{2 S}-R_{3 S} \\
\frac{d E_{a} \kappa S}{d t}=R_{1 S}-R_{2 S}+R_{4 S}-R_{10 S} \\
\frac{d E I_{a} \kappa S}{d t}=R_{7 S}+R_{9 S} \\
\frac{d E I_{a} \kappa S_{p}}{d t}=R_{8 S}-R_{9 S} \\
\frac{d E_{a} I_{S} \kappa S}{d t}=R_{10 S}+R_{11 S}+R_{16 S} \\
\frac{d E I_{S} \kappa S}{d t}=-R_{11 S}-R_{12 S}+R_{17 S} \\
\frac{d E_{a} I_{S} \kappa S_{p}}{d t}=R_{13 S}+R_{14 S}-R_{16 S} \\
\frac{d E I_{S} \kappa S_{p}}{d t}=-R_{14 S}-R_{15 S}-R_{17 S}
\end{gathered}
$$




\section{A.2.3 Model Interrogation of Insulation Mechanisms}

\section{Substrate-Competitive Inhibitor}

To fully understand the cause of the predicted substrate-competitive insulation, we further examined the effect of the scaffold efficiency on the steady state concentration of enzymesubstrate active intermediate (Figure A.4). First, this shows that increasing the scaffold efficiency number leads to more enzyme in the active intermediate at steady state. This increase in the amount of active intermediate means more inhibitor is required to overcome this increased activity, which leads to a decreased inhibitor potency on the scaffold.

\section{ATP-Competitive Inhibitor}

The prediction of insulation from ATP-competitive inhibitors was surprising, thus we sought to understand its underlying mechanism. To probe the effect of ATP-competitive inhibitors we directly changed the catalytic rate constant, $k_{c a t}$, since the action of ATP-competitive inhibitors was modeled as reducing this rate constant (Figure A.4). This analysis showed that increasing the scaffold efficiency number led to an increase in the fraction of CKAR phosphorylated at all $k_{\text {cat }}$ values, thus shifting the response to the left. The nonlinear dependence of the CKAR phosphorylation levels means that at higher $k_{c a t}$ values the CKAR phosphorylation can become less sensitive to decreases in $k_{\text {cat }}$. Thus there is a "catalytic reserve" at these saturation levels which the inhibitor would have to first overcome before significantly decreasing CKAR phosphorylation levels. Increasing the scaffold efficiency number pushes the system closer to that saturation level at the baseline $k_{c a t}$ value for the given enzyme. The decrease in the sensitivity to changes in $k_{c a t}$ can be seen by the shallower initial slope at higher $\epsilon$ values (Figure A.5B). 

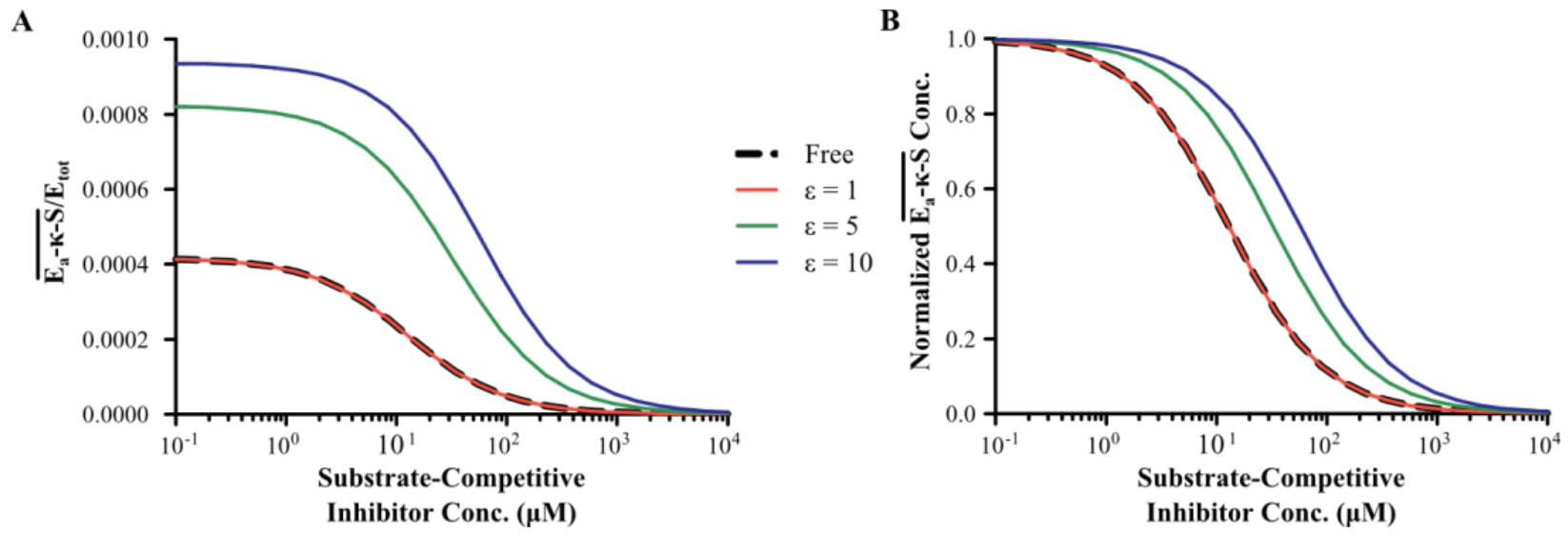

Figure A.4: Dose-dependent effects of the substrate-competitive inhibitor on the active intermediate concentration. (A) The fraction of enzyme in the active intermediate state was simulated for increasing concentrations of the substrate-competitive inhibitor for both the Free model (dashed line) and the scaffold model with increasing scaffold efficiency numbers (solid lines). (B) The responses were normalized to the active intermediate concentration in the absence of the substratecompetitive inhibitor to further illustrate the shift in the dose response with increasing scaffold efficiency.

A

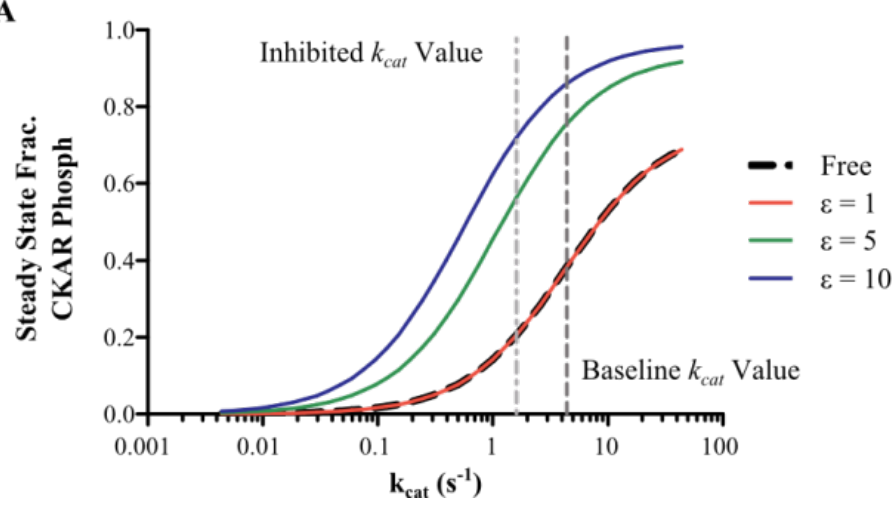

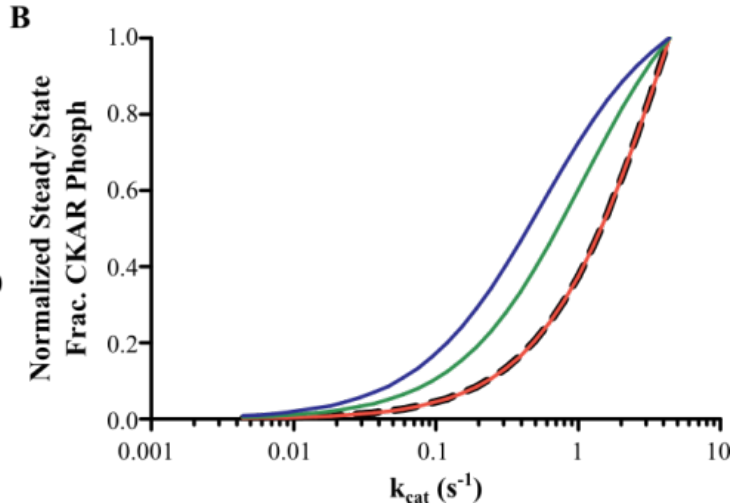

Figure A.5: The scaffold creates a "catalytic reserve," where CKAR phosphorylation is less sensitive to decreases in $k_{\text {cat }}$. (A) The steady state fraction of CKAR phosphorylated after stimulation with PDBu calculated for varying catalytic rate constant, $k_{c a t}$, for the free model (dashed line) and scaffold models (solid lines) with increasing scaffold efficiency numbers. The baseline $k_{\text {cat }}$ value used in the model $\left(4.40 \mathrm{~s}^{-1}\right)$ and the inhibited $k_{\text {cat }}$ value with $1 \mu M$ Gö6976 present $\left(1.62 s^{-1}\right)$ are shown with vertical dashed lines. (B) The CKAR responses were normalized to the fraction of CKAR phosphorylated at the baseline $k_{\text {cat }}$. 


\section{A.2.4 Model Sensitivity Analysis}

To examine how other enzymes and substrates may be affected by scaffold we varied the kinase $\left(k_{c a t}, k_{e s d}, K_{m}\right)$ and phosphatase $\left(k_{\text {ppase }}\right)$ rate constants and examined the range of scaffold effects. The ranges over which the parameters were varied was determined through extracting published kinetic rate constants for kinases and phosphatases for different substrates (Table A.5). Because there is very few published values for the enzyme substrate dissociation rate we assumed a range similar to that found for $k_{c a t}$ around the value use for the PKC-CKAR model. The first order rate constant for the phosphatase, $k_{\text {ppase }}$, was approximated by dividing the $k_{\text {cat }}$ by the $K_{m}$ for paired measurements for a given phosphatase. The parameters were linearly varied and evenly sampled across the parameter ranges (Table A.6) using Latin Hypercube Sampling (lhsdesign) with 2000 independent samples. To ensure a fair comparison across all hypothetical enzyme-scaffold-substrate complexes, the scaffold efficiency number was held constant at $\epsilon=5.7$ and the basal activity of the enzyme was set to zero. All other parameters were kept the same as the PKC-CKAR model.

For each sample the extent of amplification, acceleration and insulation by the scaffold was quantified. Amplification was quantified as the fold increase when the scaffold is present on the steady state fraction of substrate phosphorylated upon stimulation with an agonist. Acceleration was quantified by the fold decrease in the half maximal time of the agonist response on the scaffold. Insulation of the scaffold from substrate competitive inhibitors was quantified by the shift in the dose response in the presence of the scaffold. Example kinetic responses and dose response curves for high acceleration (i, green star), high insulation (ii, magenta star), and high amplification (iii, yellow star) are shown in Figure A.6. To verify that none of the individual parameters were directly responsible for the variability in the model outputs, we examined the correlation of the individual parameters to each model 
output (Figure A.7). Indeed, no single parameter is individually controlling any of the model results and the model is sensitive to each of these parameters. 


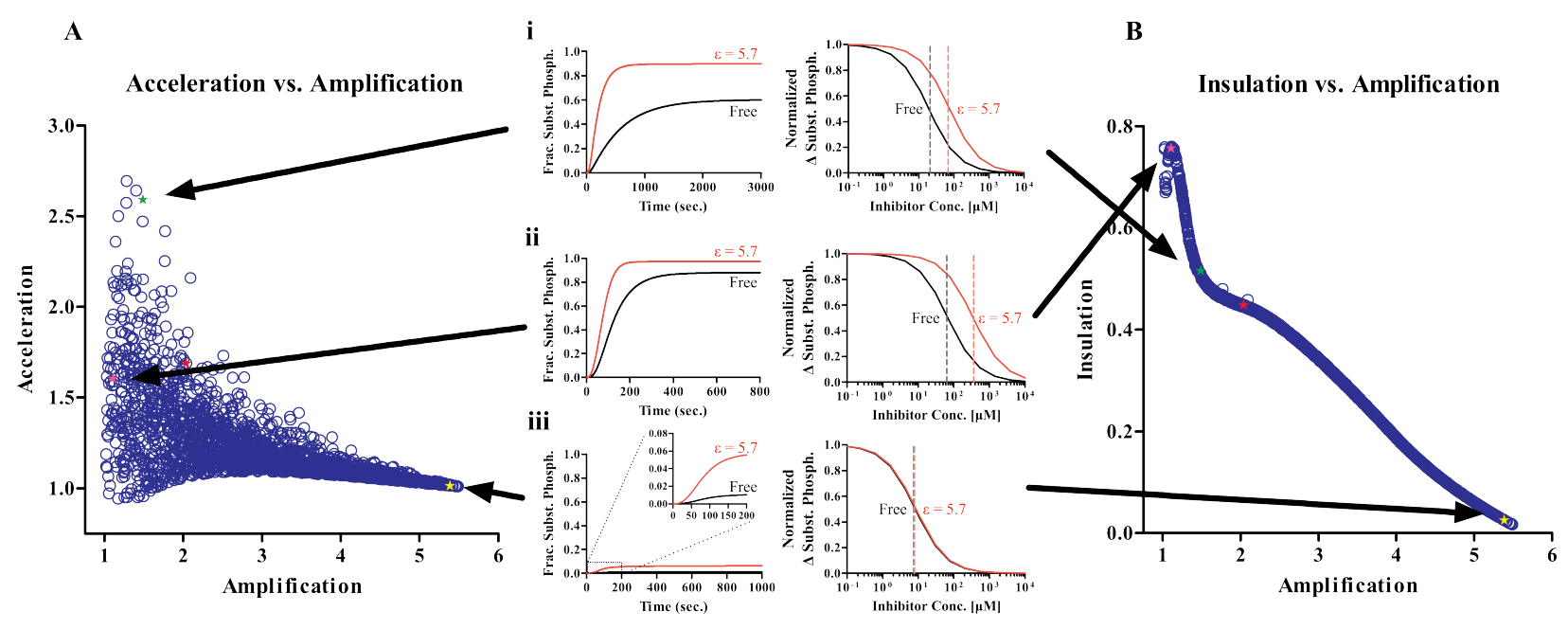

Figure A.6: Example predictions from hypothetical scaffold signaling complexes. Scatter plots show predicted amplification versus acceleration (A) and amplification versus insulation (B) of the 2000 hypothetical enzyme-scaffold-substrate complexes, with a scaffold efficiency number $\epsilon=5.7$. Three example responses are shown (i-iii) where the left plot is the time response to stimulation with an enzyme activator and the right plot is a dose response to a substrate-competitive inhibitor. For panels i-iii the black line represents the free model and the red line represents the scaffold model with a scaffold efficiency number of $\epsilon=5.7$. (i) [Green star] A signaling complex with high acceleration (2.59 fold increase) and low amplification (1.48 fold) also showed a moderately high strength of insulation (0.515 log shift). (ii) [Magenta star] A signaling complex with high insulation (0.757 log shift) also corresponded to low amplification (1.11 fold) and moderate acceleration (1.61 fold). (iii) [Yellow Star] A signaling complex with high amplification (5.39 fold) and low acceleration (1.01 fold) also corresponded to a low shift in the inhibitor sensitivity (0.025 log shift). 


\section{Model Parameters}
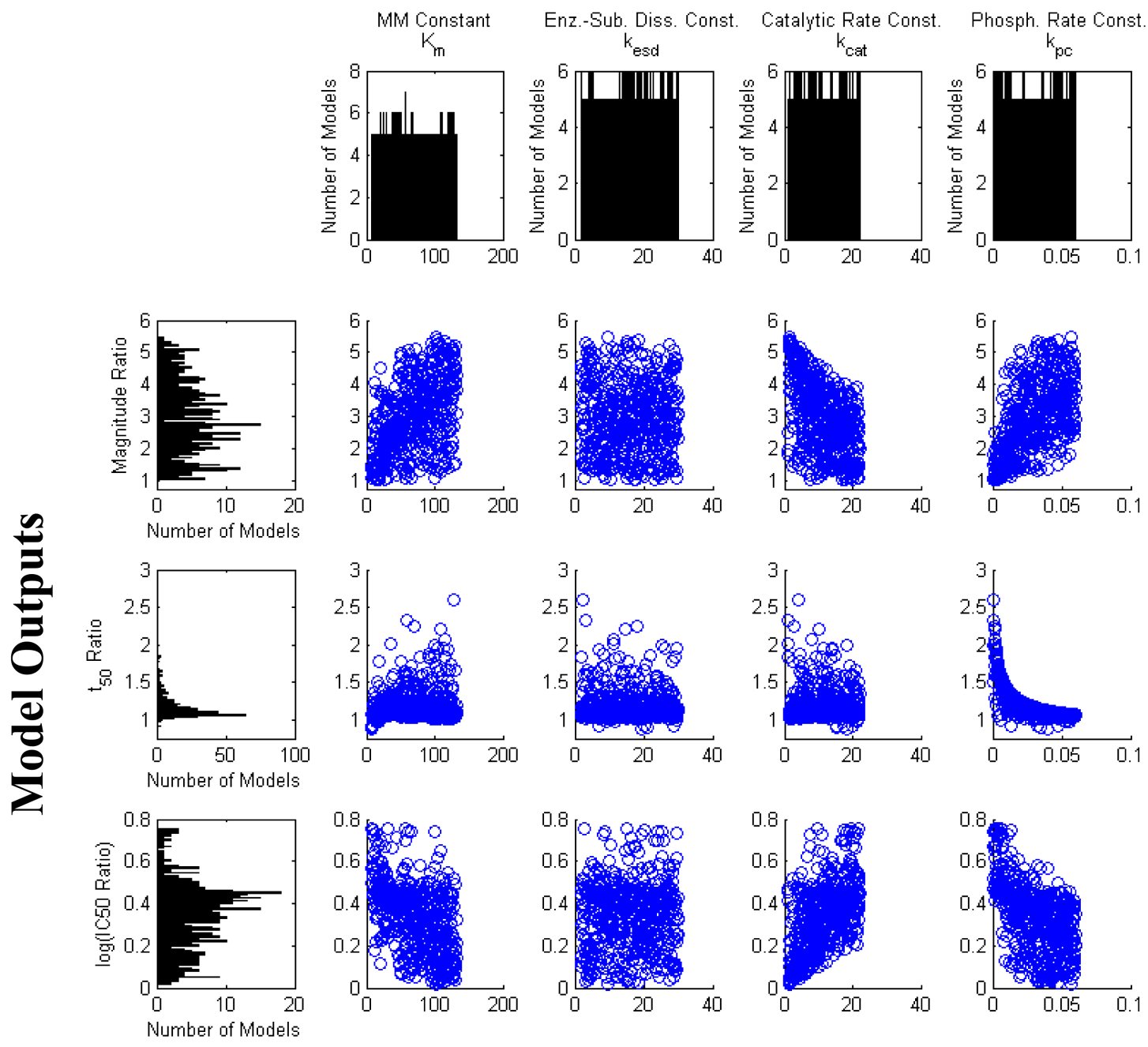

Figure A.7: Individual model parameters do not directly correlate to scaffold response. Histograms of either the model parameters (columns) or model outputs (rows) are shown in black. The magnitude ratio is the ratio of the fraction of CKAR phosphorylated on the scaffold to solution biochemistry, $S_{p}($ Scaffold $) / S_{p}$ (Free). The $t_{50}$ ratio is the ratio of the half maximal time in solution to on the scaffold, $t_{50}($ Free $) / t_{50}($ Scaffold $)$. Insulation is quantified as the $\log$ of the ratio of the $I C_{50}$ for substrate competitive inhibitors on scaffolds to solution biochemistry, $\log _{10}\left(I C_{50}(\right.$ Scaffold $) / I C_{50}$ (Free)). Scatter plots (blue) show the correlation between individual model parameters and model outputs. The $\mathrm{x}$-axis is defined by the column heading (e.g. Km) and the y-axis is defined by the row label (e.g. Magnitude Ratio). 
Table A.4: Inhibition kinetic parameters.

\begin{tabular}{|c|c|c|c|c|}
\hline Symbol & Definition & Units & Value & Source \\
\hline$K_{m}(A T P)$ & $\begin{array}{l}\text { Michaelis-menten const. } \\
\text { for ATP on PKC }\end{array}$ & $\mu M$ & 24 & [199] \\
\hline$K_{i}(G \ddot{6} 6976)$ & $\begin{array}{l}\text { Inhibition constant for } \\
\text { Gö6976 on PKC vs. ATP }\end{array}$ & $\mu M$ & 0.0028 & {$[200]$} \\
\hline$A T P$ & ATP concentration & $\mu M$ & 5000 & {$[201]$} \\
\hline$I C_{50}(P K C 20-28)$ & $\begin{array}{l}\text { half maximal inhibitory } \\
\text { concentration for PKC } \\
20-28\end{array}$ & $\mu M$ & 8 & $\begin{array}{l}\text { manufacturing } \\
\text { documents }\end{array}$ \\
\hline$k_{i a S}$ & $\begin{array}{l}\text { Enzyme-Substrate com- } \\
\text { petitive inhibitor associ- } \\
\text { ation rate }\end{array}$ & $\mu M^{-1} s^{-1}$ & 0.3860 & $\begin{array}{l}\text { assumed to be the } \\
\text { same as } k_{\text {esa }}\end{array}$ \\
\hline$k_{i d S}$ & $\begin{array}{l}\text { Enzyme-Substrate com- } \\
\text { petitive inhibitor dissoci- } \\
\text { ation rate }\end{array}$ & $s^{-1}$ & 3.0877 & $\begin{array}{l}\text { Calculated from } K_{i} \\
\text { assuming the } \\
K_{i}=I C_{50}\end{array}$ \\
\hline$I C_{50}($ Calph.C $)$ & $\begin{array}{l}\text { half maximal inhibitory } \\
\text { concentration for Calph. } \\
\text { C }\end{array}$ & $\mu M$ & 0.05 & {$[202]$} \\
\hline$k_{i a a}$ & $\begin{array}{l}\text { Enzyme-activation } \\
\text { competitive inhibitor } \\
\text { association rate }\end{array}$ & $\mu M^{-1} s^{-1}$ & 1.6229 & $\begin{array}{l}\text { assumed to be the } \\
\text { same as } k_{a a}\end{array}$ \\
\hline$k_{i d a}$ & $\begin{array}{l}\text { Enzyme-activation } \\
\text { competitive inhibitor } \\
\text { dissociation rate }\end{array}$ & $s^{-1}$ & $7.27 \cdot 10^{-4}$ & $\begin{array}{l}\text { Adjusted to agree } \\
\text { with } I C_{50}\end{array}$ \\
\hline
\end{tabular}


Table A.5: Kinase and Phosphatase kinetic parameter values.

\begin{tabular}{|ccll|}
\hline Enzyme & Parameter & Values & Units \\
\hline \multirow{2}{*}{ PKC } & $k_{\text {cat }}$ & $9.2[203], 9.7[204], 4.4[196]$ & $s^{-1}$ \\
& $K_{m}$ & $17.4-59.9[205], 132[206], 57[197]$ & $\mu M$ \\
\hline \multirow{2}{*}{ PKA } & $k_{\text {cat }}$ & $19.6[204], 20[207], 22[208], 4.18-8.6[209]$ & $s^{-1}$ \\
& $K_{m}$ & $30[207], 7.6[208], 101-136[209], 12.5-100[210]$ & $\mu M$ \\
\hline \multirow{2}{*}{ CaMKII } & $k_{\text {cat }}$ & $1.15[204]$ & $s^{-1}$ \\
& $K_{m}$ & $24[204]$ & $\mu M$ \\
\hline \multirow{2}{*}{ PP1 } & $k_{\text {cat }}$ & $5.14[211]$ & $s^{-1}$ \\
& $K_{m}$ & $1930[211]$ & $\mu M$ \\
\hline \multirow{2}{*}{ PP2A } & $k_{\text {cat }}$ & $0.77[211]$ & $s^{-1}$ \\
& $K_{m}$ & $960[211]$ & $\mu M$ \\
\hline \multirow{2}{*}{ PP2B } & $k_{\text {cat }}$ & $2.72[211]$ & $s^{-1}$ \\
& $K_{m}$ & $8680[211]$ & $\mu M$ \\
\hline \multirow{2}{*}{ PP2C } & $k_{\text {cat }}$ & $2.4[212], 0.0088-1.19[211]$ & $s^{-1}$ \\
& $K_{m}$ & $36[212], 0.223-2050[211]$ & $\mu M$ \\
\hline
\end{tabular}

Table A.6: Parameter ranges for sensitivity analysis.

\begin{tabular}{|lcl|}
\hline Parameter & Units & Range \\
\hline$k_{\text {cat }}$ & $s^{-1}$ & $1.15-22$ \\
$K_{m}($ kinase $)$ & $\mu M$ & $7.6-132$ \\
$k_{\text {esd }}$ & $s^{-1}$ & $1.76-30$ \\
$k_{\text {ppase }}$ & $s^{-1}$ & $0.00058-0.06$ \\
\hline
\end{tabular}




\section{Appendix B}

\section{AKAP5 model description}

\section{B.1 EP module}

The elecrophysiology module is adapted from the Fridlyand et al 2003 model [99].

\section{B.1.1 TEA Stimulated $\mathrm{Ca}^{2+}$ Oscillation}

We achieved TEA stimulated oscillations in the same approach done by Fridlyand et al in the 2003 model [99]. First the basal Cav 1.2 conductance was set to 400.

$$
g m V C a=400.0
$$

Then upon TEA stimulation the potassium channel fluxes are changed to the following

$$
\begin{gathered}
g m K A T P=4000.0 \\
g m K D r=800.0
\end{gathered}
$$




$$
g m K C a=60
$$

\section{B.1.2 Feedback by PKA}

To incorporate feedback we assumed that unphosphorylated $\mathrm{Ca}_{\mathrm{V}} 1.2$ had a conductance of 200 and the phosphorylated Cav1.2 had a conductance of 450. Michaelis-Menten equations were used to model the phosporylation of $\mathrm{Cav}_{\mathrm{v}} 1.2$.

$$
\begin{gathered}
R_{\text {phos-Cav }}=k_{\text {cat }}\left(P K A_{-} C a v\right) \cdot \frac{P K A a_{\text {in }} \cdot\left(C a_{V} 1.2 t o t-C a_{V} 1.2 p\right)}{K_{m}\left(P K A_{-} C a v\right)+\left(C a_{V} 1.2 t o t-C a_{V} 1.2 p\right)} \\
R_{\text {ppase-Cav }}=k_{\text {cat }}(\text { ppase_Cav }) \cdot \frac{P P t o t \cdot C a_{V} 1.2 p}{\left.K_{m}(\text { PPase_Cav })+C a_{V} 1.2 p\right)} \\
\frac{d\left(C a_{V} 1.2 p\right)}{d t}=R_{\text {phos-Cav }}-R_{\text {ppase-Cav }}
\end{gathered}
$$

In this work we tested whether the PM or AKAP5 feedback is necessary for the $\mathrm{Ca}^{2+}$ oscillations. Thus for the equations above $P K A a_{i n}$ is either $P K A a_{P M}$ or $\xi \cdot P K A a_{a k a p}$, where $\xi$ is the fold increase of PKA "effective concentration" due to AKAP tethering [45]. Here we assume $\xi=10$ as was used by Saucerman et al. in a previous model [45]. 
Table B.1: Cav1.2 Phosphorylation Parameters

\begin{tabular}{|clcc|}
\hline parameter & Name & Unit & Value \\
\hline$k_{\text {cat }}($ PKA_Cav $)$ & Catalytic rate constant for & $s^{-1}$ & $54[45]$ \\
& PKA phosph. of Cav1.2 & & \\
$K_{m}($ PKA_Cav $)$ & M.M constant for PKA & $\mu M^{-1} s^{-1}$ & 50 (Adj. from [111]) \\
& phosph. of Ca 1.2 & & \\
Ca $a_{V} 1.2 t o t$ & Total Cav1.2 Conc. & $\mu M^{-1}$ & 0.5 \\
$k_{\text {cat }}($ ppase_Cav $)$ & $\begin{array}{l}\text { Catalytic rate constant for } \\
\text { dephosph. of Cav } 1.2\end{array}$ & $s^{-1}$ & $8.5[111]$ \\
$K_{m}($ PPase_Cav $)$ & $\begin{array}{l}\text { M.M constant for dephosph. } \\
\text { of Ca } 1.2\end{array}$ & $\mu M^{-1} s^{-1}$ & $7[111]$ \\
PPtot & Total Phosphatase Conc. & $\mu M^{-1}$ & 0.3 \\
$\xi$ & Effective conc. factor & n.d. & $10[45]$ \\
\hline
\end{tabular}

\section{B.2 CaM Module}

The CaM module was directly used from the work done by Saucerman and Bers [109]. The only difference is that we reduced the calcium buffer concentration from $24 \mu M$ to $0.24 \mu M$ because $\beta$-cells do not have the extensive contractile machinery seen in cardiac cells, for which the model was developed [109].

\section{B.3 PKA Module definition}

Table B.2: Definition of model species, AKAP5 model

\begin{tabular}{|cl|}
\hline Symbol & Definition \\
\hline RC & Inactive PKA (Regulatory and catalytic subunit) \\
RC_cAMP & PKA with 1 cAMP bound \\
RC_2cAMP & PKA with 2 cAMP bound \\
R_PKA & regulatory subunit of PKA \\
PKAa & active PKA \\
\hline
\end{tabular}




\section{B.3.1 PKA equations}

The mechanisms underlying PKA activation and AKAR phosphorylation are the same for both the plasma membrane and AKAP5 compartments. The equations below describe the activation of PKA where $\mathrm{X}$ is used in place of pm or akap, for the plasma membrane or AKAP compartment respectively Figure B.1.

\section{PKA Activation}

PKA mass balance

$$
R C_{X}=R_{C t o t_{X}}-R C_{-} c A M P_{X}-P K A a_{X} ;
$$

Now we can define reaction fluxes as the reactions as defined in Figure B.X.

$$
\begin{gathered}
R_{1 X}=k_{f}\left(R C_{-} c A M P\right) \cdot R C_{X}-k_{r}\left(R C_{\_} c A M P\right) \cdot R C_{-} c A M P_{X} \\
R_{2 X}=k_{f}\left(R C \_2 c A M P\right) \cdot R C_{-} c A M P_{X}-k_{r}\left(R C \_2 c A M P\right) \cdot R C \_2 c A M P_{X}
\end{gathered}
$$

Because neither the PKA catalytic subunit or the regulatory subunit are destroyed from

either compartment, and they associate and dissociate together, their concentration will be equal.

$$
\begin{gathered}
R \_2 c A M P_{X}=P K A a_{X} \\
R_{3 X}=k_{f}\left(R \_2 c A M P\right) \cdot R C \_2 c A M P_{X}-k_{r}\left(R \_2 c A M P\right) \cdot R \_2 c A M P_{X} \cdot P K A a
\end{gathered}
$$

Therefore differential equations for PKA in each compartment can be defined by the following ODEs.

$$
\frac{d R C_{-} c A M P_{X}}{d t}=R_{1 X}-R_{2 X}
$$




$$
\begin{aligned}
\frac{d R C \_2 c A M P_{X}}{d t} & =R_{2 X}-R_{3 X} \\
\frac{d P K A a_{X}}{d t} & =R_{3 X}
\end{aligned}
$$

\section{AKAR Phosphorylation}

Similar to PKA activation, the mechanism of phosphorylation and dephosporylation are the same for both compartments. Phosporylation:

$$
R_{4 X}=k_{\text {cat }}\left(P K A_{-} A K A R\right) \cdot \frac{\text { AKARtot }_{X}-A K A R p_{X}}{K_{m}\left(P K A_{-} A K A R\right)+\left(\text { AKARtot }_{X}-A K A R p_{X}\right)}
$$

Dephosporylation:

$$
R_{5 X}=k_{\text {cat }}\left(\text { PPase } \_A K A R p\right) \cdot \frac{A K A R p_{X}}{K_{m}\left(\text { PPase }_{-} A K A R p\right)+A K A R p_{X}}
$$

Therefore the ODE is:

$$
\frac{d A K A R p_{X}}{d t}=R_{4 X}-R_{5 X}
$$

For the Phosphatase model, the $k_{\text {cat }}\left(P K A_{-} A K A R\right)$ and $k_{\text {cat }}\left(P P a s e_{-} A K A R p\right)$ were both reduced by a factor of 10 .

\section{B.3.2 cAMP degradation}

\section{Plasma Membrane}

Here we use PDE3 as a general PDE

$$
R_{6}=V m\left(P D E 3_{p m}\right) \cdot c A M P_{p m}
$$


Table B.3: PKA and AKAR Parameters

\begin{tabular}{|c|c|c|c|}
\hline parameter & Name & Unit & Value \\
\hline$k_{f}\left(R C \_c A M P\right)$ & RC-cAMP assoc. rate & $\mu M^{-1} s^{-1}$ & $1[110]$ \\
\hline$k_{r}\left(R C \_c A M P\right)$ & RC_cAMP dissoc. rate & $s^{-1}$ & $1.64[110]$ \\
\hline$k_{f}\left(R C \_2 c A M P\right)$ & RC_cAMP-cAMP assoc. rate & $\mu M^{-1} s^{-1}$ & $1[110]$ \\
\hline$k_{r}\left(R C \_2 c A M P\right)$ & RC_2cAMP dissoc. rate & $s^{-1}$ & $9.14[110]$ \\
\hline$k_{f}\left(R \_2 c A M P\right)$ & $\begin{array}{l}\text { Activation of catalytic subunit of } \\
\text { PKA }\end{array}$ & $s^{-1}$ & $4.375[110]$ \\
\hline$k_{r}\left(R \_2 c A M P\right)$ & $\begin{array}{l}\text { Regulatory subunit binding of } \\
\text { PKA }\end{array}$ & $\mu M^{-1} s^{-1}$ & $1[110]$ \\
\hline$k_{\text {cat }}\left(P K A_{-} A K A R\right)$ & $\begin{array}{l}\text { Catalytic rate constant for PKA } \\
\text { phosph. of AKAR }\end{array}$ & $s^{-1}$ & $54[45]$ \\
\hline$K_{m}\left(P K A_{-} A K A R\right)$ & $\begin{array}{l}\text { M.M constant for PKA phosph. } \\
\text { of AKAR }\end{array}$ & $\mu M^{-1} s^{-1}$ & 50 (Adj. from [111]) \\
\hline$k_{\text {cat }}\left(P P a s e_{-} A K A R p\right)$ & $\begin{array}{l}\text { Catalytic rate constant for PPase } \\
\text { dephosph. of AKARp }\end{array}$ & $s^{-1}$ & $8.5[111]$ \\
\hline$K_{m}\left(P P a s e_{-} A K A R p\right)$ & $\begin{array}{l}\text { M.M constant for PPase de- } \\
\text { phosph. of AKARp }\end{array}$ & $\mu M^{-1} s^{-1}$ & $7[111]$ \\
\hline
\end{tabular}

PDE4 is assumed to be further activated by PKA phosphorylation

$$
R_{7}=V m\left(P D E 4_{p m}\right) \cdot c A M P_{p m} \cdot\left(1+k p k a p d e 4 \cdot P K A a_{p m}\right)
$$

Finally,, we have the $\mathrm{Ca}^{2+}$ activated PDE1

$$
R_{8}=k_{c}(P D E 1) \cdot \frac{\left(K 3 C a \cdot\left(C a^{2+}\right)^{3}+K 4 C a \cdot\left(C a^{2+}\right)^{4}\right)}{k p k a p d e 1 c+P K A a_{p m}} \cdot c A M P_{p m}
$$

\section{AKAP5}

Here we use PDE3 as a general PDE

$$
R_{9}=V m\left(P D E 3_{a k a p}\right) \cdot c A M P_{a k a p}
$$


PDE4 is assumed to be further activated by PKA phosphorylation

$$
R_{10}=V m\left(P D E 4_{a k a p}\right) \cdot c A M P_{a k a p} \cdot\left(1+k p k a p d e 4 \cdot P K A a_{a k a p}\right)
$$

We assume that the AKAP5 compartment does not have PDE1

Table B.4: PDE Parameters

\begin{tabular}{|clllll|}
\hline parameter & Unit & Ni & cAMP & PPase & AC8/AC5 \\
& & $\mathbf{2 0 1 1}$ & Diff. & & \\
\hline$V m\left(P D E 3_{\text {pm }}\right)$ & $s^{-1}$ & 0.01 & 0.005 & 0.9 & 0.2 \\
$V m\left(P D E 4_{p m}\right)$ & $s^{-1}$ & N/A & 0 & 0 & 0 \\
$k p k a p d e 4$ & $\mu M^{-1}$ & N/A & $100[21]$ & $100[21]$ & $100[21]$ \\
$k_{c}(P D E 1)$ & & $1[21]$ & $1[21]$ & 1.5 & $1[21]$ \\
$K 3 C a$ & $\left(\mu M^{3} s\right)^{-1}$ & 0.072 & $0.072[21]$ & $0.072[21]$ & $0.072[21]$ \\
& & {$[21]$} & & & \\
$K 4 C a$ & $\left(\mu M^{3} s\right)^{-1}$ & $2.16[21]$ & $2.16[21]$ & $2.16[21]$ & $2.16[21]$ \\
$k p k a p d e 1 c$ & $\mu M^{-1}$ & N/A & $0.01[21]$ & $0.01[21]$ & $0.01[21]$ \\
$V m\left(P D E 3_{\text {akap }}\right)$ & $s^{-1}$ & N/A & 0.1 & 0.9 & 0.9 \\
$V m\left(P D E 4_{\text {akap }}\right)$ & $s^{-1}$ & N/A & 0.1 & 0.9 & 0.9 \\
\hline
\end{tabular}

\section{B.3.3 cAMP Production}

Ni Model: Constant AC activity [21]

$$
R_{11}=\operatorname{Vacm}
$$

For all the other models we have at AC8 creating cAMP in some compartment. AC8 is assumed to be activated by CaM when activated by $\mathrm{Ca}^{2+}$ and bound to AKAP5.

$$
R_{12}=k_{\text {cat }}(A C 8) \frac{a C a M_{-} A K A P 5}{a C a M_{-} A K A P 5+E C_{50}\left(A C 8_{-} C a\right)} \frac{A T P t o t}{A T P t o t+K_{m}\left(A C 8_{-} A T P\right)}
$$


On the other hand AC5 activity is dependent on the displacement of PKC from AKAP5 by CaM. Because the PKC and CaM displace each other, the concentration of AKAP5 bound $\mathrm{CaM}$ is equal to that the released and assumed active PKC.

$$
P K C=a C a M_{-} A K A P 5
$$

The PKA phosphorylates AC5 via the following reaction rate:

$$
R_{13}=k_{\text {cat }}\left(P K C \_A C 5\right) \cdot P K C \cdot \frac{(A C 5 t o t-A C 5 p)}{K_{m}(P K C)+(A C 5 t o t-A C 5 p)}
$$

We used our previous model [104] examining PKC activity as the basis for these kinetic parameters and similarly, we will use a linear rate of dephosphorylation of this PKC substrate.

$$
R_{14}=k_{\text {ppase }}(A C 5) \cdot A C 5 p
$$

The ODE of phosphorylated is then the following equation

$$
\frac{d A C 5 p}{d t}=R_{13}-R_{14}
$$

Finally, $A C 5 p$ derived cAMP is given by the following equation

$$
R_{15}=k_{c a t}(A C 5) \cdot A C 5 p \cdot \frac{A T P t o t}{A T P t o t+K_{m}\left(A C 5_{-} A T P\right)}
$$


Table B.5: AC Parameters

\begin{tabular}{|clcl|}
\hline parameter & Name & Unit & Value \\
\hline Vacm & cAMP constant production rate & $\mu M s^{-1}$ & $0.002[21]$ \\
$k_{\text {cat }}(A C 8)$ & catalytic rate cAMP by AC8 & $s^{-1}$ & $59[213]$ \\
$E C_{50}\left(A C 8 \_C a\right)$ & $\begin{array}{l}\text { concentration for 50\% activation } \\
\text { of AC8 by Ca }\end{array}$ & $\mu M$ & $0.56[115]$ \\
ATPtot & total ATP concentration & $\mu M$ & 1000 \\
$K_{m}\left(A C 8 \_A T P\right)$ & MM rate const. for cAMP prod & $\mu M$ & $340[213]$ \\
& by AC8 & & \\
$k_{\text {cat }}\left(P K C \_A C 5\right)$ & catalytic rate of PKC phosph of & $s^{-1}$ & $4.4[104]$ \\
& AC5 & & \\
$K_{m}(P K C)$ & M.M. of PKC phosph of AC5 & $\mu M$ & $57[104]$ \\
$k_{\text {pase }}(A C 5)$ & linear dephosp. rate of AC5 & $s^{-1}$ & 0.0024 \\
& & & {$[104]$ ) (adj. } \\
$k_{\text {cat }}(A C 5)$ & catalytic rate cAMP by AC5 & $s^{-1}$ & $59 \quad$ (assumed \\
$K_{m}\left(A C 5 \_A T P\right)$ & MM rate const. for cAMP prod & $\mu M$ & $340 \quad$ (assumed \\
& by AC5 & & same as AC8) \\
\hline
\end{tabular}

\section{B.3.4 Other Signaling}

\section{CaM Binding to AKAP5}

As discussed above, activated CaM binds AKAP5 and displaces PKC. This equation describes the binding of CaM to AKAP5

$$
\begin{aligned}
& R_{16}= \\
& \qquad \begin{array}{r}
k_{a}\left(C a M_{-} A K A P\right) \cdot\left(a C a M-a C a M_{-} A K A P 5\right) \cdot\left(A K A P t o t-a C a M_{-} A K A P 5\right) \\
-k_{d}\left(C a M_{-} A K A P\right) \cdot a C a M_{-} A K A P 5
\end{array}
\end{aligned}
$$


Where the ODE for the $a C a M_{-} A K A P 5$ is,

$$
\frac{d a C a M_{\_} A K A P 5}{d t}=R_{16}
$$

\section{cAMP diffusion}

The diffusion of cAMP between compartments for the cAMP Diffusion model was based off of models by Bhalla and Upinder [63]. The flux from the AKAP to the cytosol is governed by this equation, which is written in terms of moles/time

$$
R_{17}=-k_{f}(c A M P) \cdot c A M P_{a k a p}+k_{b}(c A M P) \cdot c A M P_{p m}
$$

The difference in the volumes of the compartments we must incorporate this difference into the ODEs, by dividing by the volume of the compartment that is being considered, $V_{c y t o}$ and $V_{\text {akap }}$.

\section{B.3.5 cAMP ODEs}

Now with all the other reactions defined we can write out the cAMP ODEs.

\section{AKAP}

In all the AKAP models AC8 is the only source of cAMP so the ODE can be written as

$$
\frac{d c A M P_{\text {akap }}}{d t}=R_{12}-R_{9}-R_{10}-R_{1 a k a p}-R_{2 a k a p}+\frac{R_{17}}{V_{a k a p}}
$$

PM

The plasma membrane on the other hand has different sources of cAMP 
Table B.6: Misc. Parameters. Model parameters for Calmodulin, cAMP diffusion and compartment volumes. The values for each model are listed under their respective name. v.f. - volume fraction

\begin{tabular}{|c|c|c|c|c|c|c|}
\hline parameter & Name & Unit & $\begin{array}{l}\mathrm{Ni} \\
2011\end{array}$ & $\begin{array}{l}\text { cAMP } \\
\text { Diff. }\end{array}$ & PPase & $\begin{array}{l}\mathrm{AC} 8 / \\
\mathrm{AC5}\end{array}$ \\
\hline$k_{a}\left(C a M \_A K A P\right)$ & $\begin{array}{l}\text { assoc. const of } \\
\text { CaM for AKAP79 } \\
\text { (with calcium) }\end{array}$ & $\mu M^{-1} s^{-1}$ & $\mathrm{~N} / \mathrm{A}$ & $\begin{array}{l}0.203 \\
{[122]}\end{array}$ & $\begin{array}{l}0.203 \\
{[122]}\end{array}$ & $\begin{array}{l}0.203 \\
{[122]}\end{array}$ \\
\hline$k_{d}\left(C a M_{-} A K A P\right)$ & $\begin{array}{l}\text { dissassoc. const. of } \\
\text { CaM for AKAP79 } \\
\text { (with calcium) }\end{array}$ & $s^{-1}$ & $\mathrm{~N} / \mathrm{A}$ & $\begin{array}{l}0.0055 \\
{[122]}\end{array}$ & $\begin{array}{l}0.0055 \\
{[122]}\end{array}$ & $\begin{array}{l}0.0055 \\
{[122]}\end{array}$ \\
\hline$k_{f}(c A M P)$ & $\begin{array}{l}\text { Rate of diff. from } \\
\text { AKAP to Cyto }\end{array}$ & $\mu m^{3} s^{-1}$ & $\mathrm{~N} / \mathrm{A}$ & $\begin{array}{l}509.1970 \\
\text { (calcu- } \\
\text { lated } \\
D=20)\end{array}$ & $\mathrm{N} / \mathrm{A}$ & $\mathrm{N} / \mathrm{A}$ \\
\hline$k_{b}(c A M P)$ & $\begin{array}{l}\text { Rate of diff. from } \\
\text { AKAP to Cyto }\end{array}$ & $\mu m^{3} s^{-1}$ & $\mathrm{~N} / \mathrm{A}$ & $\begin{array}{l}509.1970 \\
(\text { calcu- } \\
\text { lated } \\
D=20)\end{array}$ & $\mathrm{N} / \mathrm{A}$ & $\mathrm{N} / \mathrm{A}$ \\
\hline$V_{\text {cyto }}$ & $\begin{array}{l}\text { Volume of the } \\
\text { plasma membrane } \\
\text { compartment }\end{array}$ & $\mu m^{3}$ & $\mathrm{~N} / \mathrm{A}$ & $\begin{array}{l}1.6503 \times 10^{4} \\
(\text { v.f. }=0.5)\end{array}$ & $\mathrm{N} / \mathrm{A}$ & $\begin{array}{l}3.2676 \times 10^{4} \\
(\text { v.f. }=0.99)\end{array}$ \\
\hline$V_{a k a p}$ & $\begin{array}{l}\text { Volume of the } \\
\text { AKAP compart- } \\
\text { ment }\end{array}$ & $\mu m^{3}$ & $\mathrm{~N} / \mathrm{A}$ & $\begin{array}{l}1.6503 \times 10^{4} \\
\text { (v.f.=0.5) }\end{array}$ & $\mathrm{N} / \mathrm{A}$ & $\begin{array}{l}330.0636 \\
\text { (v.f. }=0.01)\end{array}$ \\
\hline
\end{tabular}

$$
c A M P \_i n_{p m}(\text { Model })= \begin{cases}R_{11} & \text { if Model is Ni } 2011 \\ 0 & \text { if Model is cAMP diffusion } \\ R_{12} & \text { if Model is Phosphatase } \\ R_{15} & \text { if Model is AC8/AC5 }\end{cases}
$$

Using this the PM cAMP ODE is

$$
\frac{d c A M P_{p m}}{d t}=c A M P_{\imath} i n_{p m}(\text { Model })-R_{6}-R_{7}-R_{8}-R_{1 p m}-R_{2 p m}-\frac{R_{17}}{V_{p m}}
$$




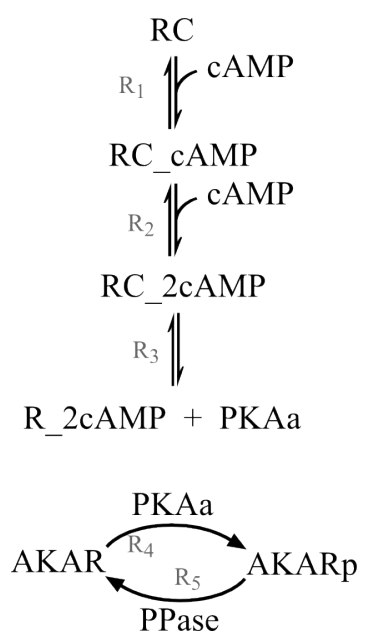

Figure B.1: Details of PKA signaling in the model. Reaction annotations (gray R)

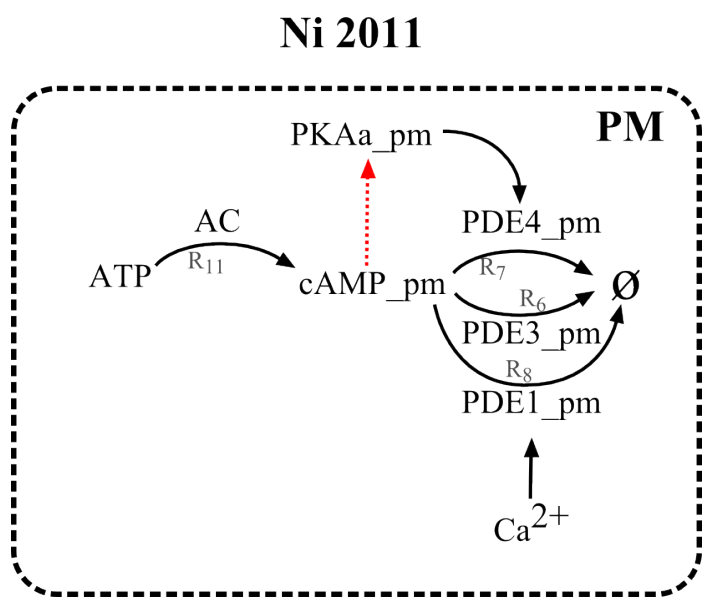

Figure B.2: Details of the Ni 2011 signaling model. Red arrows indicate mechanisms that are explained in more detail elsewhere, Figure B.1 


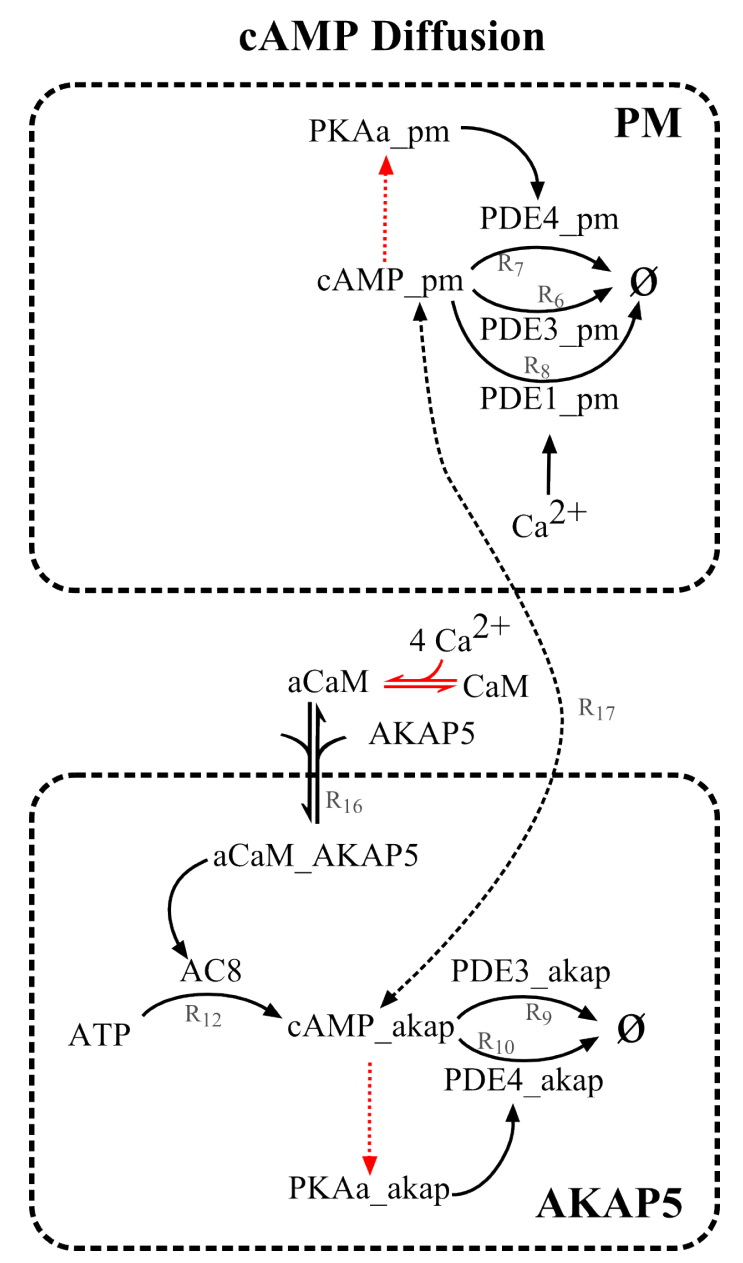

Figure B.3: Details of the cAMP Diffusion signaling model. Red arrows indicate mechanisms that are explained in more detail elsewhere, Figure B.1 and [109] 


\section{Phosphatase}

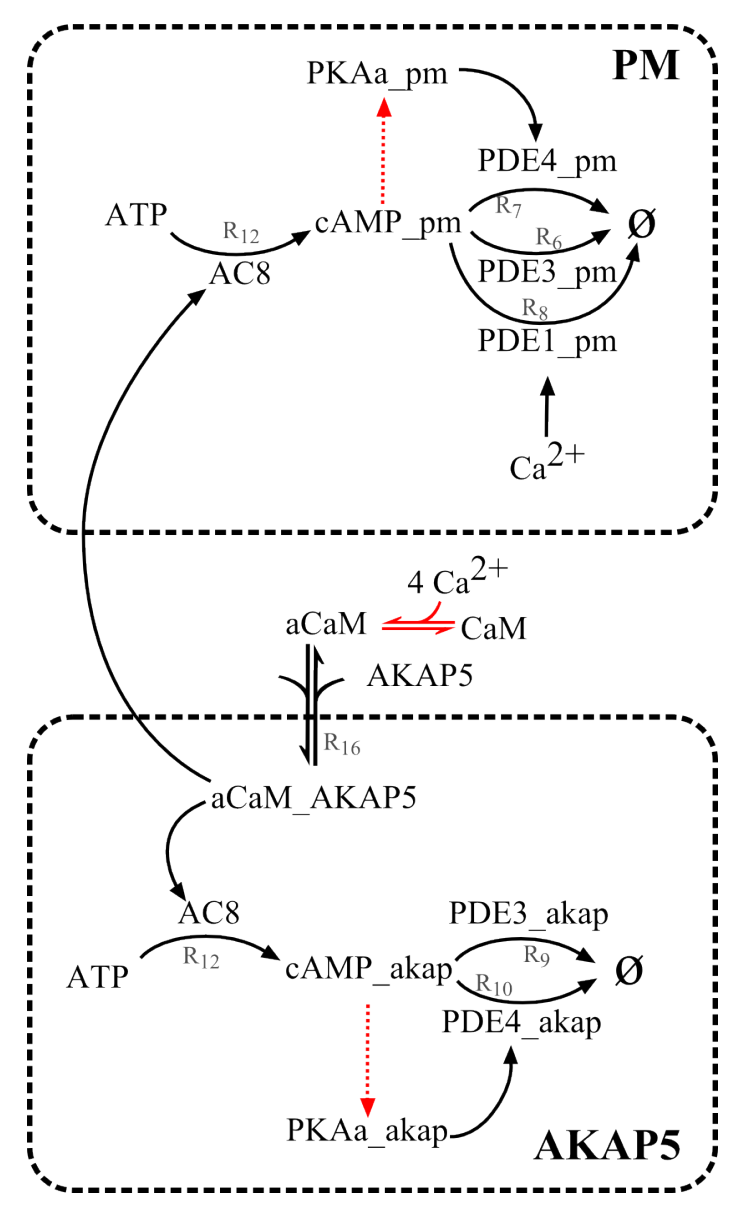

Figure B.4: Details of the Phosphatase signaling model. Red arrows indicate mechanisms that are explained in more detail elsewhere, Figure B.1 and [109] 


\section{$\mathrm{AC8} / \mathrm{AC5}$}

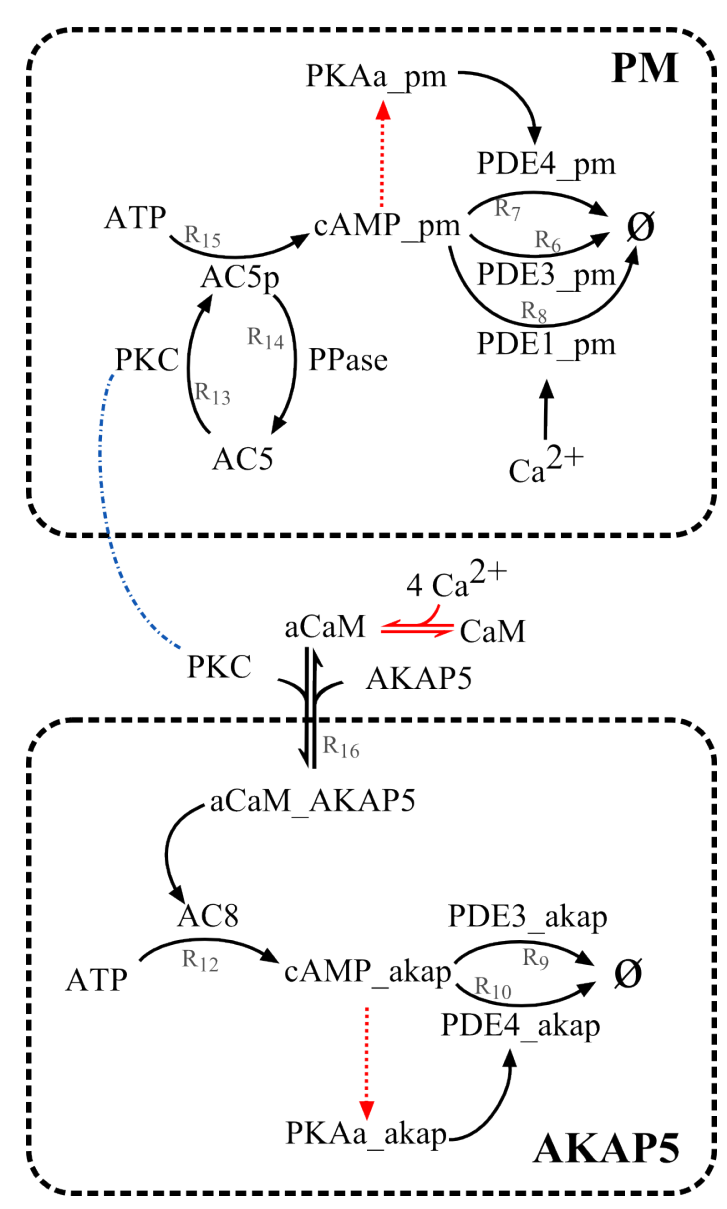

Figure B.5: Details of the AC8/AC5 signaling model. Red arrows indicate mechanisms that are explained in more detail elsewhere, Figure B.1 and [109] 


\section{Bibliography}

[1] T. Pawson and J. Scott, "Signaling Through Scaffold, Anchoring, and Adaptor Proteins," Science, vol. 278, pp. 2075-2080, Dec. 1997.

[2] W. Wong and J. D. Scott, "AKAP signalling complexes: focal points in space and time.," Nature reviews. Molecular cell biology, vol. 5, pp. 959-70, Dec. 2004.

[3] M. S. Kapiloff and K. D. Chandrasekhar, "A-kinase anchoring proteins: temporal and spatial regulation of intracellular signal transduction in the cardiovascular system.," Journal of cardiovascular pharmacology, vol. 58, pp. 337-8, Oct. 2011.

[4] J. J. C. Michel and J. D. Scott, "AKAP mediated signal transduction.," Annual review of pharmacology and toxicology, vol. 42, pp. 235-57, Jan. 2002.

[5] K. L. Dodge-Kafka, A. Bauman, and M. S. Kapiloff, "A-kinase anchoring proteins as the basis for cAMP signaling.," Handbook of experimental pharmacology, pp. 3-14, Jan. 2008.

[6] M. L. Ruehr, M. a. Russell, and M. Bond, "A-kinase anchoring protein targeting of protein kinase A in the heart.," Journal of molecular and cellular cardiology, vol. 37, pp. 653-65, Sept. 2004. 
[7] H. H. Patel, L. L. Hamuro, B. J. Chun, Y. Kawaraguchi, A. Quick, B. Rebolledo, J. Pennypacker, J. Thurston, N. Rodriguez-Pinto, C. Self, G. Olson, P. a. Insel, W. R. Giles, S. S. Taylor, and D. M. Roth, "Disruption of protein kinase A localization using a trans-activator of transcription (TAT)-conjugated A-kinase-anchoring peptide reduces cardiac function.," The Journal of biological chemistry, vol. 285, pp. 27632-40, Sept. 2010.

[8] W. G. Tingley, L. Pawlikowska, J. G. Zaroff, T. Kim, T. Nguyen, S. G. Young, K. Vranizan, P.-Y. Kwok, M. A. Whooley, and B. R. Conklin, "Gene-trapped mouse embryonic stem cell-derived cardiac myocytes and human genetics implicate AKAP10 in heart rhythm regulation.," Proceedings of the National Academy of Sciences of the United States of America, vol. 104, pp. 8461-6, May 2007.

[9] S. O. Marx, J. Kurokawa, S. Reiken, H. Motoike, J. D'Armiento, A. R. Marks, and R. S. Kass, "Requirement of a macromolecular signaling complex for beta adrenergic receptor modulation of the KCNQ1-KCNE1 potassium channel.," Science (New York, N.Y.), vol. 295, pp. 496-9, Jan. 2002.

[10] G. K. Carnegie, J. Soughayer, F. D. Smith, B. S. Pedroja, F. Zhang, D. Diviani, M. R. Bristow, M. T. Kunkel, A. C. Newton, L. K. Langeberg, and J. D. Scott, "AKAP-Lbc mobilizes a cardiac hypertrophy signaling pathway.," Molecular cell, vol. 32, pp. 16979, Oct. 2008.

[11] M. Wirtenberger, J. Schmutzhard, K. Hemminki, A. Meindl, C. Sutter, R. K. Schmutzler, B. Wappenschmidt, M. Kiechle, N. Arnold, B. H. F. Weber, D. Niederacher, C. R. Bartram, and B. Burwinkel, "The functional genetic variant Ile646Val located in the 
kinase binding domain of the A-kinase anchoring protein 10 is associated with familial breast cancer.," Carcinogenesis, vol. 28, pp. 423-6, Feb. 2007.

[12] J. H. Yang and J. J. Saucerman, "Computational models reduce complexity and accelerate insight into cardiac signaling networks.," Circulation research, vol. 108, pp. 85-97, Jan. 2011.

[13] K. W. Trotter, I. D. Fraser, G. K. Scott, M. J. Stutts, J. D. Scott, and S. L. Milgram, "Alternative splicing regulates the subcellular localization of A-kinase anchoring protein 18 isoforms.," The Journal of cell biology, vol. 147, pp. 1481-92, Dec. 1999.

[14] L. J. Huang, L. Wang, Y. Ma, K. Durick, G. Perkins, T. J. Deerinck, M. H. Ellisman, and S. S. Taylor, "NH2-Terminal targeting motifs direct dual specificity A-kinaseanchoring protein 1 (D-AKAP1) to either mitochondria or endoplasmic reticulum.," The Journal of cell biology, vol. 145, pp. 951-9, May 1999.

[15] A. K. Gillingham and S. Munro, "The PACT domain, a conserved centrosomal targeting motif in the coiled-coil proteins AKAP450 and pericentrin.," EMBO reports, vol. 1, pp. 524-9, Dec. 2000.

[16] M. S. Kapiloff, R. V. Schillace, A. M. Westphal, and J. D. Scott, "mAKAP: an A-kinase anchoring protein targeted to the nuclear membrane of differentiated myocytes.," Journal of cell science, vol. 112 ( Pt 1, pp. 2725-36, Aug. 1999.

[17] D. Carr, D. DeManno, A. Atwood, M. Hunzicker-Dunn, and J. Scott, "Folliclestimulating hormone regulation of A-kinase anchoring proteins in granulosa cells," J. Biol. Chem., vol. 268, pp. 20729-20732, Oct. 1993. 
[18] A. A. Paulucci-Holthauzen, L. A. Vergara, L. J. Bellot, D. Canton, J. D. Scott, and K. L. O'Connor, "Spatial distribution of protein kinase A activity during cell migration is mediated by A-kinase anchoring protein AKAP Lbc.," The Journal of biological chemistry, vol. 284, pp. 5956-67, Feb. 2009.

[19] K. J. Newhall, A. R. Criniti, C. S. Cheah, K. C. Smith, K. E. Kafer, A. D. Burkart, and G. S. McKnight, "Dynamic anchoring of PKA is essential during oocyte maturation.," Current biology : CB, vol. 16, pp. 321-7, Feb. 2006.

[20] W. R. Burack and a. S. Shaw, "Signal transduction: hanging on a scaffold.," Current opinion in cell biology, vol. 12, pp. 211-6, Apr. 2000.

[21] Q. Ni, A. Ganesan, N.-N. Aye-Han, X. Gao, M. D. Allen, A. Levchenko, and J. Zhang, "Signaling diversity of PKA achieved via a Ca(2+)-cAMP-PKA oscillatory circuit.," Nature chemical biology, vol. 7, pp. 34-40, Jan. 2011.

[22] M. J. Kraeutler, A. R. Soltis, and J. J. Saucerman, "Modeling cardiac beta-adrenergic signaling with normalized-Hill differential equations: comparison with a biochemical model.," BMC systems biology, vol. 4, p. 157, Nov. 2010.

[23] A. Zeke, M. Lukács, W. a. Lim, and A. Reményi, "Scaffolds: interaction platforms for cellular signalling circuits.," Trends in cell biology, vol. 19, pp. 364-74, Aug. 2009.

[24] K. L. Dodge-Kafka, J. Soughayer, G. C. Pare, J. J. Carlisle Michel, L. K. Langeberg, M. S. Kapiloff, and J. D. Scott, "The protein kinase A anchoring protein mAKAP coordinates two integrated cAMP effector pathways.," Nature, vol. 437, pp. 574-8, Sept. 2005. 
[25] M. S. Kapiloff, L. A. Piggott, R. Sadana, J. Li, L. A. Heredia, E. Henson, R. Efendiev, and C. W. Dessauer, "An adenylyl cyclase-mAKAPbeta signaling complex regulates cAMP levels in cardiac myocytes.," The Journal of biological chemistry, vol. 284, pp. 23540-6, Aug. 2009.

[26] N. Hoshi, L. K. Langeberg, C. M. Gould, A. C. Newton, and J. D. Scott, "Interaction with AKAP79 modifies the cellular pharmacology of PKC.," Molecular cell, vol. 37, pp. 541-50, Feb. 2010.

[27] J. Zhang, Y. Ma, S. S. Taylor, and R. Y. Tsien, "Genetically encoded reporters of protein kinase A activity reveal impact of substrate tethering.," Proceedings of the National Academy of Sciences of the United States of America, vol. 98, pp. 149975002, Dec. 2001.

[28] C. Indolfi, E. Stabile, C. Coppola, A. Gallo, C. Perrino, G. Allevato, L. Cavuto, D. Torella, E. Di Lorenzo, G. Troncone, A. Feliciello, E. V. Avvedimento, and M. Chiariello, "Membrane-Bound Protein Kinase A Inhibits Smooth Muscle Cell Proliferation In Vitro and In Vivo by Amplifying cAMP-Protein Kinase A Signals," Circ. Res., vol. 88, no. 3, pp. 319-324, 2001.

[29] A. Feliciello, Y. Li, E. V. Avvedimento, M. E. Gottesman, and C. S. Rubin, "A-kinase anchor protein 75 increases the rate and magnitude of cAMP signaling to the nucleus," Current Biology, vol. 7, pp. 1011-1014, Dec. 1997.

[30] G. K. Carnegie, F. D. Smith, G. McConnachie, L. K. Langeberg, and J. D. Scott, "AKAP-Lbc nucleates a protein kinase D activation scaffold.," Molecular cell, vol. 15, pp. 889-99, Sept. 2004. 
[31] K. Scott and C. S. Zuker, "Assembly of the Drosophila phototransduction cascade into a signalling complex shapes elementary responses.," Nature, vol. 395, pp. 805-8, Oct. 1998.

[32] T. Gao, A. Yatani, M. L. Dell'Acqua, H. Sako, S. a. Green, N. Dascal, J. D. Scott, and M. M. Hosey, "cAMP-dependent regulation of cardiac L-type Ca2+ channels requires membrane targeting of PKA and phosphorylation of channel subunits.," Neuron, vol. 19, pp. 185-96, July 1997.

[33] S. J. Tavalin, "AKAP79 Selectively Enhances Protein Kinase C Regulation of GluR1 at a Ca2+-Calmodulin-dependent Protein Kinase II/Protein Kinase C Site," The Journal of biological chemistry, vol. 283, pp. 11445-11452, Apr. 2008.

[34] A. Persechini, K. McMillan, and P. Leakey, "Activation of myosin light chain kinase and nitric oxide synthase activities by calmodulin fragments," J. Biol. Chem., vol. 269, no. 23, pp. 16148-16154, 1994.

[35] R. Milo, S. Shen-Orr, S. Itzkovitz, N. Kashtan, D. Chklovskii, and U. Alon, "Network motifs: simple building blocks of complex networks.," Science (New York, N.Y.), vol. 298, pp. 824-7, Oct. 2002.

[36] L. Zhang, B. E. Blackman, M. D. Schonemann, T. Zogovic-Kapsalis, X. Pan, M. Tagliaferri, H. A. Harris, I. Cohen, R. A. R. Pera, S. H. Mellon, R. I. Weiner, and D. C. Leitman, "Estrogen receptor beta-selective agonists stimulate calcium oscillations in human and mouse embryonic stem cell-derived neurons.," PloS one, vol. 5, p. e11791, Jan. 2010.

[37] V. K. Mutalik and K. V. Venkatesh, "Theoretical Biology and Medical Quantification of the glycogen cascade system : the ultrasensitive responses of liver glycogen syn- 
thase and muscle phosphorylase are due to distinctive regulatory designs," Theoretical Biology and Medical Modelling, vol. 18, pp. 1-18, 2005.

[38] W. Ma, A. Trusina, H. El-Samad, W. a. Lim, and C. Tang, "Defining network topologies that can achieve biochemical adaptation.," Cell, vol. 138, pp. 760-73, Aug. 2009.

[39] A. Hoffmann, A. Levchenko, M. L. Scott, and D. Baltimore, "The IkappaB-NF-kappaB signaling module: temporal control and selective gene activation.," Science (New York, N.Y.), vol. 298, pp. 1241-5, Nov. 2002.

[40] B. Novák and J. J. Tyson, "Design principles of biochemical oscillators.," Nature reviews. Molecular cell biology, vol. 9, pp. 981-91, Dec. 2008.

[41] L. B. Lester, M. C. Faux, J. B. Nauert, and J. D. Scott, "Targeted Protein Kinase A and PP-2B Regulate Insulin Secretion through Reversible Phosphorylation," Endocrinology, vol. 142, pp. 1218-1227, Mar. 2001.

[42] A. L. Bauman, J. Soughayer, B. T. Nguyen, D. Willoughby, G. K. Carnegie, W. Wong, N. Hoshi, L. K. Langeberg, D. M. F. Cooper, C. W. Dessauer, and J. D. Scott, "Dynamic regulation of cAMP synthesis through anchored PKA-adenylyl cyclase V/VI complexes.," Molecular cell, vol. 23, pp. 925-31, Sept. 2006.

[43] D. E. Koshland, a. Goldbeter, and J. B. Stock, "Amplification and adaptation in regulatory and sensory systems.," Science (New York, N.Y.), vol. 217, pp. 220-5, July 1982.

[44] J. E. Ferrell Jr., "Tripping the switch fantastic: how a protein kinase cascade can convert graded inputs into switch-like outputs," Trends in Biochemical Sciences, vol. 21, pp. 460-466, Dec. 1996. 
[45] J. J. Saucerman, L. L. Brunton, A. P. Michailova, and A. D. McCulloch, "Modeling beta-adrenergic control of cardiac myocyte contractility in silico.," The Journal of biological chemistry, vol. 278, pp. 47997-48003, Nov. 2003.

[46] A. Singh, J. M. J. Redden, M. S. M. Kapiloff, and K. L. Dodge-Kafka, "The large isoforms of AKAP18 mediate the phosphorylation of Inhibitor-1 by PKA and the inhibition of PP1 activity.," Molecular pharmacology, vol. 79, pp. 533-540, Dec. 2011.

[47] L. K. Langeberg and J. D. Scott, "A-kinase-anchoring proteins.," Journal of cell science, vol. 118, pp. 3217-20, Aug. 2005.

[48] J. J. Saucerman and A. D. McCulloch, "Mechanistic systems models of cell signaling networks: a case study of myocyte adrenergic regulation.," Progress in biophysics and molecular biology, vol. 85, no. 2-3, pp. 261-78, 2004.

[49] J. J. Saucerman, S. N. Healy, M. E. Belik, J. L. Puglisi, and A. D. McCulloch, "Proarrhythmic consequences of a KCNQ1 AKAP-binding domain mutation: computational models of whole cells and heterogeneous tissue.," Circulation research, vol. 95, pp. 1216-24, Dec. 2004.

[50] D. Darbar, D. M. Roden, M. F. Ali, T. Yang, and M. S. Wathen, "Images in cardiovascular medicine. Himalayan T waves in the congenital long-QT syndrome.," Circulation, vol. 111, p. e161, Mar. 2005.

[51] O. Andrei and M. Calder, "A Model and Analysis of the AKAP Scaffold," Electronic Notes in Theoretical Computer Science, pp. 1-14, 2010.

[52] A. Levchenko, J. Bruck, and P. W. Sternberg, "Scaffold proteins may biphasically affect the levels of mitogen-activated protein kinase signaling and reduce its threshold 
properties.," Proceedings of the National Academy of Sciences of the United States of America, vol. 97, pp. 5818-23, May 2000.

[53] E. C. O'Shaughnessy, S. Palani, J. J. Collins, C. A. Sarkar, and E. C. O'Shaughnessy, "Tunable Signal Processing in Synthetic MAP Kinase Cascades," Cell, vol. 144, pp. 119-131, Jan. 2011.

[54] J. W. Locasale, A. S. Shaw, and A. K. Chakraborty, "Scaffold proteins confer diverse regulatory properties to protein kinase cascades.," Proceedings of the National Academy of Sciences of the United States of America, vol. 104, pp. 13307-12, Aug. 2007.

[55] J. D. Scott and T. Pawson, "Cell signaling in space and time: where proteins come together and when they're apart.," Science (New York, N.Y.), vol. 326, pp. 1220-1224, Nov. 2009.

[56] A. Kiyatkin, E. Aksamitiene, N. I. Markevich, N. M. Borisov, J. B. Hoek, and B. N. Kholodenko, "Scaffolding protein Grb2-associated binder 1 sustains epidermal growth factor-induced mitogenic and survival signaling by multiple positive feedback loops.," The Journal of biological chemistry, vol. 281, pp. 19925-38, July 2006.

[57] W. S. Hlavacek, J. R. Faeder, M. L. Blinov, A. S. Perelson, and B. Goldstein, "The complexity of complexes in signal transduction.," Biotechnology and bioengineering, vol. 84, pp. 783-94, Dec. 2003.

[58] J. R. Faeder, W. S. Hlavacek, I. Reischl, M. L. Blinov, H. Metzger, A. Redondo, C. Wofsy, and B. Goldstein, "Investigation of early events in Fc epsilon RI-mediated signaling using a detailed mathematical model.," Journal of immunology (Baltimore, Md. : 1950), vol. 170, pp. 3769-81, Apr. 2003. 
[59] N. M. Borisov, N. I. Markevich, J. B. Hoek, and B. N. Kholodenko, "Signaling through receptors and scaffolds: independent interactions reduce combinatorial complexity.," Biophysical journal, vol. 89, pp. 951-66, Aug. 2005.

[60] N. M. Borisov, N. I. Markevich, J. B. Hoek, and B. N. Kholodenko, "Trading the micro-world of combinatorial complexity for the macro-world of protein interaction domains.," Bio Systems, vol. 83, no. 2-3, pp. 152-66, 2006.

[61] M. W. Sneddon, J. R. Faeder, and T. Emonet, "Efficient modeling, simulation and coarse-graining of biological complexity with NFsim," Nature Methods, vol. 8, pp. 177183, Feb. 2011.

[62] A. Eldar and M. B. Elowitz, "Functional roles for noise in genetic circuits," Nature, vol. 467, pp. 167-173, Sept. 2010.

[63] U. S. Bhalla, "Signaling in small subcellular volumes. I. Stochastic and diffusion effects on individual pathways.," Biophysical journal, vol. 87, pp. 733-44, Aug. 2004.

[64] N.-N. Aye-Han, Q. Ni, and J. Zhang, "Fluorescent biosensors for real-time tracking of post-translational modification dynamics.," Current opinion in chemical biology, vol. 13, pp. 392-7, Oct. 2009.

[65] L. M. DiPilato, X. Cheng, and J. Zhang, "Fluorescent indicators of cAMP and Epac activation reveal differential dynamics of cAMP signaling within discrete subcellular compartments.," Proceedings of the National Academy of Sciences of the United States of America, vol. 101, pp. 16513-8, Nov. 2004. 
[66] M. D. Allen and J. Zhang, "Subcellular dynamics of protein kinase A activity visualized by FRET-based reporters.," Biochemical and biophysical research communications, vol. 348, pp. 716-21, Sept. 2006.

[67] V. Sample, L. M. Dipilato, J. H. Yang, Q. Ni, J. J. Saucerman, and J. Zhang, "Regulation of nuclear PKA revealed by spatiotemporal manipulation of cyclic AMP.," Nature chemical biology, vol. 8, pp. 375-382, Feb. 2012.

[68] C. Li, M. Donizelli, N. Rodriguez, H. Dharuri, L. Endler, V. Chelliah, L. Li, E. He, A. Henry, M. I. Stefan, J. L. Snoep, M. Hucka, N. Le Novère, and C. Laibe, "BioModels Database: An enhanced, curated and annotated resource for published quantitative kinetic models.," BMC systems biology, vol. 4, p. 92, Jan. 2010.

[69] G. D. Bader, M. P. Cary, and C. Sander, "Pathguide: a pathway resource list.," Nucleic acids research, vol. 34, pp. D504-6, Jan. 2006.

[70] C. M. Lloyd, J. R. Lawson, P. J. Hunter, and P. F. Nielsen, "The CellML Model Repository.," Bioinformatics (Oxford, England), vol. 24, pp. 2122-3, Sept. 2008.

[71] U. S. Bhalla and R. Iyengar, "Emergent Properties of Networks of Biological Signaling Pathways," Science, vol. 283, pp. 381-387, Jan. 1999.

[72] A. R. Soltis and J. J. Saucerman, "Robustness portraits of diverse biological networks conserved despite order-of-magnitude parameter uncertainty.," Bioinformatics (Oxford, England), vol. 27, pp. 2888-94, Oct. 2011.

[73] I. I. Moraru, J. C. Schaff, B. M. Slepchenko, M. L. Blinov, F. Morgan, A. Lakshminarayana, F. Gao, Y. Li, and L. M. Loew, "Virtual Cell modelling and simulation software environment.," IET systems biology, vol. 2, pp. 352-62, Sept. 2008. 
[74] M. A. Rizzo, G. Springer, K. Segawa, W. R. Zipfel, and D. W. Piston, "Optimization of pairings and detection conditions for measurement of FRET between cyan and yellow fluorescent proteins.," Microscopy and microanalysis, vol. 12, pp. 238-54, June 2006.

[75] L. L. Gallegos, M. T. Kunkel, and A. C. Newton, "Targeting protein kinase C activity reporter to discrete intracellular regions reveals spatiotemporal differences in agonistdependent signaling.," The Journal of biological chemistry, vol. 281, pp. 30947-56, Oct. 2006.

[76] A. Periasamy, H. Wallrabe, Y. Chen, and M. Barroso, "Chapter 22: Quantitation of protein-protein interactions: confocal FRET microscopy.," Methods in cell biology, vol. 89, pp. 569-98, Jan. 2008.

[77] T. Zal and N. R. J. Gascoigne, "Photobleaching-corrected FRET efficiency imaging of live cells.," Biophysical journal, vol. 86, pp. 3923-39, June 2004.

[78] J. J. Saucerman, J. Zhang, J. C. Martin, L. X. Peng, A. E. Stenbit, R. Y. Tsien, and A. D. McCulloch, "Systems analysis of PKA-mediated phosphorylation gradients in live cardiac myocytes.," Proceedings of the National Academy of Sciences of the United States of America, vol. 103, pp. 12923-8, Aug. 2006.

[79] J. D. Violin, J. Zhang, R. Y. Tsien, and A. C. Newton, "A genetically encoded fluorescent reporter reveals oscillatory phosphorylation by protein kinase C.," The Journal of cell biology, vol. 161, pp. 899-909, June 2003.

[80] L. H. Tay, O. Griesbeck, and D. T. Yue, "Live-cell transforms between Ca2+ transients and FRET responses for a troponin-C-based Ca2+ sensor.," Biophysical journal, vol. 93, pp. 4031-40, Dec. 2007. 
[81] J. D. Scott, C. W. Dessauer, K. Tasken, and K. Taskén, "Creating Order from Chaos: Cellular Regulation by Kinase Anchoring.," Annual review of pharmacology and toxicology, vol. 53, pp. 1-24, Oct. 2012.

[82] A. S. Edwards and J. D. Scott, "A-kinase anchoring proteins: protein kinase A and beyond," Current Opinion in Cell Biology, vol. 12, pp. 217-221, Apr. 2000.

[83] S.-H. Park, A. Zarrinpar, and W. A. Lim, "Rewiring MAP kinase pathways using alternative scaffold assembly mechanisms.," Science (New York, N.Y.), vol. 299, pp. 1061-4, Feb. 2003.

[84] E. C. Greenwald and J. J. Saucerman, "Bigger, better, faster: principles and models of AKAP anchoring protein signaling.," Journal of cardiovascular pharmacology, vol. 58, pp. 462-9, Nov. 2011.

[85] G. Pidoux and K. Taskén, "Specificity and spatial dynamics of protein kinase A signaling organized by A-kinase-anchoring proteins.," Journal of molecular endocrinology, vol. 44, pp. 271-84, May 2010.

[86] J. Redden, A. Le, A. Singh, K. Federkiewicz, S. Smith, and K. Dodge-Kafka, "Spatiotemporal regulation of PKC via interactions with AKAP7 isoforms," Biochemical Journal, vol. 309, pp. 301-309, 2012.

[87] L. Michaelis, M. L. Menten, K. A. Johnson, and R. S. Goody, "The original Michaelis constant: translation of the 1913 Michaelis-Menten paper.," Biochemistry, vol. 50, pp. 8264-9, Oct. 2011. 
[88] M. C. Faux, E. N. Rollins, A. S. Edwards, L. K. Langeberg, A. C. Newton, and J. D. Scott, "Mechanism of A-kinase-anchoring protein 79 (AKAP79) and protein kinase C interaction.," The Biochemical journal, vol. 343 Pt 2, pp. 443-52, Oct. 1999.

[89] J. L. Sanderson and M. L. Dell'Acqua, "AKAP signaling complexes in regulation of excitatory synaptic plasticity.," The Neuroscientist : a review journal bringing neurobiology, neurology and psychiatry, vol. 17, pp. 321-36, June 2011.

[90] L. B. Lester, "Anchoring of protein kinase A facilitates hormone-mediated insulin secretion," Proceedings of the National Academy of Sciences, vol. 94, pp. 14942-14947, Dec. 1997.

[91] C. C. Malbon, J. Tao, E. Shumay, and H.-Y. Wang, "AKAP (A-kinase anchoring protein) domains: beads of structure-function on the necklace of G-protein signalling," Biochemical Society transactions, vol. 32, pp. 861-4, Nov. 2004.

[92] R. F. Oliveira, A. Terrin, G. Di Benedetto, R. C. Cannon, W. Koh, M. Kim, M. Zaccolo, and K. T. Blackwell, "The role of type 4 phosphodiesterases in generating microdomains of cAMP: large scale stochastic simulations.," PloS one, vol. 5, p. e11725, Jan. 2010.

[93] M. C. Good, J. G. Zalatan, and W. a. Lim, "Scaffold Proteins: Hubs for Controlling the Flow of Cellular Information," Science, vol. 332, pp. 680-686, May 2011.

[94] M. Good, G. Tang, J. Singleton, A. Reményi, and W. A. Lim, "The Ste5 scaffold directs mating signaling by catalytically unlocking the Fus3 MAP kinase for activation.," Cell, vol. 136, pp. 1085-97, Mar. 2009. 
[95] F. Hohendanner, A. D. McCulloch, L. A. Blatter, and A. P. Michailova, "Calcium and IP3 dynamics in cardiac myocytes: experimental and computational perspectives and approaches.," Frontiers in pharmacology, vol. 5, p. 35, Jan. 2014.

[96] E. Smedler and P. Uhlén, "Frequency decoding of calcium oscillations.," Biochimica et biophysica acta, vol. 1840, pp. 964-9, Mar. 2014.

[97] R. Bertram, A. Sherman, and L. S. Satin, "Metabolic and electrical oscillations: partners in controlling pulsatile insulin secretion.," American journal of physiology. Endocrinology and metabolism, vol. 293, pp. E890-900, Oct. 2007.

[98] T. Tsuboi, G. da Silva Xavier, G. G. Holz, L. S. Jouaville, A. P. Thomas, and G. A. Rutter, "Glucagon-like peptide-1 mobilizes intracellular Ca2+ and stimulates mitochondrial ATP synthesis in pancreatic MIN6 beta-cells," The Biochemical journal, vol. 369, pp. 287-99, Jan. 2003.

[99] L. E. Fridlyand, N. Tamarina, and L. H. Philipson, "Modeling of Ca2+ flux in pancreatic beta-cells: role of the plasma membrane and intracellular stores." American journal of physiology. Endocrinology and metabolism, vol. 285, pp. E138-54, July 2003.

[100] S. Mehta, N.-N. Aye-Han, A. Ganesan, L. Oldach, K. Gorshkov, and J. Zhang, "Calmodulin-controlled spatial decoding of oscillatory Ca2+ signals by calcineurin.," eLife, vol. 3, p. e03765, Jan. 2014.

[101] S. M. P. Jacobo, M. L. Guerra, and G. H. Hockerman, "Cav1.2 and Cav1.3 Are Differentially Coupled to Glucagon-Like Peptide-1 Potentiation of Glucose-Stimulated Insulin Secretion in the Pancreatic beta-Cell Line INS-1," Journal of Pharmacology and Experimental Therapeutics, vol. 331, pp. 724-732, Aug. 2009. 
[102] J. Striessnig, A. Pinggera, G. Kaur, G. Bock, and P. Tuluc, "L-type Ca(2+) channels in heart and brain.," Wiley interdisciplinary reviews. Membrane transport and signaling, vol. 3, pp. 15-38, Mar. 2014.

[103] L. E. Fridlyand, M. C. Harbeck, M. W. Roe, and L. H. Philipson, "Regulation of cAMP dynamics by $\mathrm{Ca} 2+$ and $\mathrm{G}$ protein-coupled receptors in the pancreatic beta-cell: a computational approach.," American journal of physiology. Cell physiology, vol. 293, pp. C1924-33, Dec. 2007.

[104] E. C. Greenwald, J. M. Redden, K. L. Dodge-Kafka, and J. J. Saucerman, "Scaffold state switching amplifies, accelerates, and insulates protein kinase $\mathrm{C}$ signaling.," The Journal of biological chemistry, vol. 289, pp. 2353-60, Jan. 2014.

[105] R. Efendiev, B. K. Samelson, B. T. Nguyen, P. V. Phatarpekar, F. Baameur, J. D. Scott, and C. W. Dessauer, "AKAP79 interacts with multiple adenylyl cyclase (AC) isoforms and scaffolds AC5 and -6 to alpha-amino-3-hydroxyl-5-methyl-4isoxazole-propionate (AMPA) receptors.," The Journal of biological chemistry, vol. 285, pp. 14450-8, May 2010.

[106] S. F. Oliveria, M. L. Dell'Acqua, and W. A. Sather, "AKAP79/150 anchoring of calcineurin controls neuronal L-type Ca2+ channel activity and nuclear signaling.," Neuron, vol. 55, pp. 261-75, July 2007.

[107] N. Hoshi, L. K. Langeberg, and J. D. Scott, "Distinct enzyme combinations in AKAP signalling complexes permit functional diversity.," Nature cell biology, vol. 7, pp. 106673, Nov. 2005.

[108] C. Depry, M. D. Allen, and J. Zhang, "Visualization of PKA activity in plasma membrane microdomains.," Molecular bioSystems, vol. 7, pp. 52-8, Jan. 2011. 
[109] J. J. Saucerman and D. M. Bers, "Calmodulin mediates differential sensitivity of CaMKII and calcineurin to local Ca2+ in cardiac myocytes.," Biophysical journal, vol. 95, pp. 4597-612, Nov. 2008.

[110] J. H. Yang and J. J. Saucerman, "Phospholemman is a negative feed-forward regulator of $\mathrm{Ca} 2+$ in $\beta$-adrenergic signaling, accelerating $\beta$-adrenergic inotropy.," Journal of molecular and cellular cardiology, vol. 52, pp. 1048-55, May 2012.

[111] J. H. Yang, R. K. Polanowska-Grabowska, J. S. Smith, C. W. Shields, and J. J. Saucerman, "PKA catalytic subunit compartmentation regulates contractile and hypertrophic responses to $\beta$-adrenergic signaling.," Journal of molecular and cellular cardiology, vol. 66, pp. 83-93, Jan. 2014.

[112] J. Akerboom, N. Carreras Calderón, L. Tian, S. Wabnig, M. Prigge, J. Tolö, A. Gordus, M. B. Orger, K. E. Severi, J. J. Macklin, R. Patel, S. R. Pulver, T. J. Wardill, E. Fischer, C. Schüler, T.-W. Chen, K. S. Sarkisyan, J. S. Marvin, C. I. Bargmann, D. S. Kim, S. Kügler, L. Lagnado, P. Hegemann, A. Gottschalk, E. R. Schreiter, and L. L. Looger, "Genetically encoded calcium indicators for multi-color neural activity imaging and combination with optogenetics.," Frontiers in molecular neuroscience, vol. 6, p. 2, Jan. 2013.

[113] J. T. Hulme, T. W.-C. Lin, R. E. Westenbroek, T. Scheuer, and W. A. Catterall, "Betaadrenergic regulation requires direct anchoring of PKA to cardiac CaV1.2 channels via a leucine zipper interaction with A kinase-anchoring protein 15.," Proceedings of the National Academy of Sciences of the United States of America, vol. 100, pp. 13093-8, Oct. 2003. 
[114] J. Zhang, C. J. Hupfeld, S. S. Taylor, J. M. Olefsky, and R. Y. Tsien, "Insulin disrupts beta-adrenergic signalling to protein kinase A in adipocytes.," Nature, vol. 437, pp. 569-73, Sept. 2005.

[115] N. Masada, A. Ciruela, D. A. Macdougall, and D. M. F. Cooper, "Distinct mechanisms of regulation by Ca2+/calmodulin of type 1 and 8 adenylyl cyclases support their different physiological roles.," The Journal of biological chemistry, vol. 284, pp. 445163, Feb. 2009.

[116] D. Delmeire, D. Flamez, S. A. Hinke, J. J. Cali, D. Pipeleers, and F. Schuit, "Type VIII adenylyl cyclase in rat beta cells: coincidence signal detector/generator for glucose and GLP-1.," Diabetologia, vol. 46, pp. 1383-93, Oct. 2003.

[117] L. R. Landa, M. Harbeck, K. Kaihara, O. Chepurny, K. Kitiphongspattana, O. Graf, V. O. Nikolaev, M. J. Lohse, G. G. Holz, and M. W. Roe, "Interplay of Ca2+ and cAMP signaling in the insulin-secreting MIN6 beta-cell line.," The Journal of biological chemistry, vol. 280, pp. 31294-302, Sept. 2005.

[118] I. Delint-Ramirez, D. Willoughby, G. V. R. Hammond, L. J. Ayling, and D. M. F. Cooper, "Palmitoylation targets AKAP79 protein to lipid rafts and promotes its regulation of calcium-sensitive adenylyl cyclase type 8.," The Journal of biological chemistry, vol. 286, pp. 32962-75, Sept. 2011.

[119] D. Willoughby, N. Masada, S. Wachten, M. Pagano, M. L. Halls, K. L. Everett, a. Ciruela, and D. M. F. Cooper, "AKAP79/150 Interacts with AC8 and Regulates Ca2+dependent cAMP Synthesis in Pancreatic and Neuronal Systems," Journal of Biological Chemistry, vol. 285, pp. 20328-20342, Apr. 2010. 
[120] J. J. Saucerman, E. C. Greenwald, and R. Polanowska-Grabowska, "Mechanisms of cyclic AMP compartmentation revealed by computational models.," The Journal of general physiology, vol. 143, pp. 39-48, Jan. 2014.

[121] M. G. Gold, F. Stengel, P. J. Nygren, C. R. Weisbrod, J. E. Bruce, C. V. Robinson, D. Barford, and J. D. Scott, "Architecture and dynamics of an A-kinase anchoring protein 79 (AKAP79) signaling complex.," Proceedings of the National Academy of Sciences of the United States of America, vol. 108, pp. 6426-31, Apr. 2011.

[122] M. C. Faux and J. D. Scott, "Regulation of the AKAP79-Protein Kinase C Interaction by Ca2+/Calmodulin," Journal of Biological Chemistry, vol. 272, pp. 17038-17044, July 1997.

[123] J. Kawabe, G. Iwami, T. Ebina, S. Ohno, T. Katada, Y. Ueda, C. J. Homcy, and Y. Ishikawa, "Differential activation of adenylyl cyclase by protein kinase $\mathrm{C}$ isoenzymes.," J. Biol. Chem., vol. 269, pp. 16554-16558, June 1994.

[124] A. Sculptoreanu, E. Rotman, M. Takahashi, T. Scheuer, and W. A. Catterall, "Voltagedependent potentiation of the activity of cardiac L-type calcium channel alpha 1 subunits due to phosphorylation by cAMP-dependent protein kinase.," Proceedings of the National Academy of Sciences, vol. 90, pp. 10135-10139, Nov. 1993.

[125] H. Dou, C. Wang, X. Wu, L. Yao, X. Zhang, S. Teng, H. Xu, B. Liu, Q. Wu, Q. Zhang, M. Hu, Y. Wang, L. Wang, Y. Wu, S. Shang, X. Kang, L. Zheng, J. Zhang, M. Raoux, J. Lang, Q. Li, J. Su, X. Yu, L. Chen, and Z. Zhou, "Calcium influx activates adenylyl cyclase 8 for sustained insulin secretion in rat pancreatic beta cells.," Diabetologia, vol. 58, pp. 324-33, Feb. 2015. 
[126] O. Dyachok, Y. Isakov, J. Så getorp, and A. Tengholm, "Oscillations of cyclic AMP in hormone-stimulated insulin-secreting beta-cells.," Nature, vol. 439, pp. 349-52, Jan. 2006.

[127] M. E. Doyle and J. M. Egan, "Mechanisms of action of glucagon-like peptide 1 in the pancreas.," Pharmacology \& therapeutics, vol. 113, pp. 546-93, Mar. 2007.

[128] X. Wang, J. Zhou, M. E. Doyle, and J. M. Egan, "Glucagon-like peptide-1 causes pancreatic duodenal homeobox-1 protein translocation from the cytoplasm to the nucleus of pancreatic beta-cells by a cyclic adenosine monophosphate/protein kinase Adependent mechanism.," Endocrinology, vol. 142, pp. 1820-7, May 2001.

[129] B. E. Peercy and A. S. Sherman, "How pancreatic beta-cells discriminate long and short timescale cAMP signals.," Biophysical journal, vol. 99, pp. 398-406, July 2010.

[130] P. Lipp and G. Reither, "Protein kinase C: the "masters" of calcium and lipid.," Cold Spring Harbor perspectives in biology, vol. 3, July 2011.

[131] M. L. Halls and D. M. F. Cooper, "Regulation by Ca2+-signaling pathways of adenylyl cyclases.," Cold Spring Harbor perspectives in biology, vol. 3, p. a004143, Jan. 2011.

[132] M.-L. Dequéant, E. Glynn, K. Gaudenz, M. Wahl, J. Chen, A. Mushegian, and O. Pourquié, "A complex oscillating network of signaling genes underlies the mouse segmentation clock.," Science (New York, N.Y.), vol. 314, pp. 1595-8, Dec. 2006.

[133] A. B. Reddy, N. A. Karp, E. S. Maywood, E. A. Sage, M. Deery, J. S. O’Neill, G. K. Y. Wong, J. Chesham, M. Odell, K. S. Lilley, C. P. Kyriacou, and M. H. Hastings, "Circadian orchestration of the hepatic proteome.," Current biology : CB, vol. 16, pp. 110715, June 2006. 
[134] M. T. Laub and W. F. Loomis, "A Molecular Network That Produces Spontaneous Oscillations in Excitable Cells of Dictyostelium," Molecular Biology of the Cell, vol. 9, pp. 3521-3532, Dec. 1998.

[135] C.-C. Wang, S. S. Bajikar, L. Jamal, K. A. Atkins, and K. A. Janes, "A time- and matrix-dependent TGFBR3-JUND-KRT5 regulatory circuit in single breast epithelial cells and basal-like premalignancies.," Nature cell biology, vol. 16, pp. 345-56, Apr. 2014.

[136] M. Zaccolo, "Spatial control of cAMP signalling in health and disease.," Current opinion in pharmacology, vol. 11, pp. 649-55, Dec. 2011.

[137] J. E. Ferrell, T. Y.-C. Tsai, and Q. Yang, "Modeling the cell cycle: why do certain circuits oscillate?," Cell, vol. 144, pp. 874-85, Mar. 2011.

[138] L. Eliasson, "SUR1 Regulates PKA-independent cAMP-induced Granule Priming in Mouse Pancreatic B-cells," The Journal of General Physiology, vol. 121, pp. 181-197, Feb. 2003.

[139] H. Hatakeyama, T. Kishimoto, T. Nemoto, H. Kasai, and N. Takahashi, "Rapid glucose sensing by protein kinase A for insulin exocytosis in mouse pancreatic islets.," The Journal of physiology, vol. 570, pp. 271-82, Jan. 2006.

[140] P. Rorsman and E. Renström, "Insulin granule dynamics in pancreatic beta cells.," Diabetologia, vol. 46, pp. 1029-45, Aug. 2003.

[141] J. Gromada, J. J. Holst, and P. Rorsman, "Cellular regulation of islet hormone secretion by the incretin hormone glucagon-like peptide 1," Pflugers Archiv European Journal of Physiology, vol. 435, pp. 583-594, Mar. 1998. 
[142] T. C. Rich, K. J. Webb, and S. J. Leavesley, "Can we decipher the information content contained within cyclic nucleotide signals?," The Journal of general physiology, vol. 143, pp. 17-27, Jan. 2014.

[143] C. J. Bashor, N. C. Helman, S. Yan, and W. A. Lim, "Using engineered scaffold interactions to reshape MAP kinase pathway signaling dynamics.," Science (New York, N.Y.), vol. 319, pp. 1539-43, Mar. 2008.

[144] M. Weitz, J. Kim, K. Kapsner, E. Winfree, E. Franco, and F. C. Simmel, "Diversity in the dynamical behaviour of a compartmentalized programmable biochemical oscillator.," Nature chemistry, vol. 6, pp. 295-302, Apr. 2014.

[145] P. C. Havugimana, G. T. Hart, T. Nepusz, H. Yang, A. L. Turinsky, Z. Li, P. I. Wang, D. R. Boutz, V. Fong, S. Phanse, M. Babu, S. A. Craig, P. Hu, C. Wan, J. Vlasblom, V.-u.-N. Dar, A. Bezginov, G. W. Clark, G. C. Wu, S. J. Wodak, E. R. M. Tillier, A. Paccanaro, E. M. Marcotte, and A. Emili, "A census of human soluble protein complexes.," Cell, vol. 150, pp. 1068-81, Aug. 2012.

[146] C. B. Nichols, C. F. Rossow, M. F. Navedo, R. E. Westenbroek, W. A. Catterall, L. F. Santana, and G. S. McKnight, "Sympathetic stimulation of adult cardiomyocytes requires association of AKAP5 with a subpopulation of L-type calcium channels.," Circulation research, vol. 107, pp. 747-56, Sept. 2010.

[147] B. J. Tunquist, N. Hoshi, E. S. Guire, F. Zhang, K. Mullendorff, L. K. Langeberg, J. Raber, and J. D. Scott, "Loss of AKAP150 perturbs distinct neuronal processes in mice.," Proceedings of the National Academy of Sciences of the United States of America, vol. 105, pp. 12557-62, Aug. 2008. 
[148] K. J. Schimenti, S. K. Feuer, L. B. Griffin, N. R. Graham, C. A. Bovet, S. Hartford, J. Pendola, C. Lessard, J. C. Schimenti, and J. O. Ward, "AKAP9 is essential for spermatogenesis and sertoli cell maturation in mice.," Genetics, vol. 194, pp. 447-57, June 2013.

[149] G. K. Carnegie, C. K. Means, and J. D. Scott, "A-kinase anchoring proteins: from protein complexes to physiology and disease.," IUBMB life, vol. 61, pp. 394-406, Apr. 2009.

[150] A. J. Whitmarsh and W. A, "The JIP family of MAPK scaffold proteins," Biochemical Society transactions, vol. 34, pp. 828-32, Nov. 2006.

[151] M. D. Brown and D. B. Sacks, "Protein scaffolds in MAP kinase signalling.," Cellular signalling, vol. 21, pp. 462-9, Apr. 2009.

[152] M. Sheng and E. Kim, "The Shank family of scaffold proteins," J. Cell Sci., vol. 113, pp. 1851-1856, June 2000.

[153] E. Kim and M. Sheng, "PDZ domain proteins of synapses.," Nature reviews. Neuroscience, vol. 5, pp. 771-81, Oct. 2004.

[154] X. Zhan, T. S. Kaoud, K. N. Dalby, and V. V. Gurevich, "Nonvisual arrestins function as simple scaffolds assembling the MKK4-JNK3 $\alpha 2$ signaling complex.," Biochemistry, vol. 50, pp. 10520-9, Dec. 2011.

[155] R. P. Bhattacharyya, A. Reményi, M. C. Good, C. J. Bashor, A. M. Falick, and W. A. Lim, "The Ste5 scaffold allosterically modulates signaling output of the yeast mating pathway.," Science (New York, N.Y.), vol. 311, pp. 822-6, Feb. 2006. 
[156] J. Liu and D. L. Brautigan, "Glycogen synthase association with the striated muscle glycogen-targeting subunit of protein phosphatase-1. Synthase activation involves scaffolding regulated by beta-adrenergic signaling.," The Journal of biological chemistry, vol. 275, pp. 26074-81, Aug. 2000.

[157] D. L. Brautigan, "Protein Ser/Thr phosphatases-the ugly ducklings of cell signalling.," The FEBS journal, vol. 280, pp. 324-45, Jan. 2013.

[158] W. Sents, E. Ivanova, C. Lambrecht, D. Haesen, and V. Janssens, "The biogenesis of active protein phosphatase $2 \mathrm{~A}$ holoenzymes: a tightly regulated process creating phosphatase specificity.," The FEBS journal, vol. 280, pp. 644-61, Jan. 2013.

[159] F. Herzog, A. Kahraman, D. Boehringer, R. Mak, A. Bracher, T. Walzthoeni, A. Leitner, M. Beck, F.-U. F.-U. F.-U. Hartl, N. Ban, L. Malmström, R. Aebersold, and L. Malmstrom, "Structural probing of a protein phosphatase $2 \mathrm{~A}$ network by chemical cross-linking and mass spectrometry.," Science (New York, N.Y.), vol. 337, pp. 134852, Sept. 2012.

[160] L. K. Langeberg and J. D. Scott, "Signalling scaffolds and local organization of cellular behaviour.," Nature reviews. Molecular cell biology, vol. advance on, Mar. 2015.

[161] R. Janknecht and T. Hunter, "Transcriptional control: Versatile molecular glue," Current Biology, vol. 6, pp. 951-954, Aug. 1996.

[162] H. M. Chan and N. B. La Thangue, "p300/CBP proteins: HATs for transcriptional bridges and scaffolds," J. Cell Sci., vol. 114, pp. 2363-2373, July 2001.

[163] S. Sharma, G. M. Findlay, H. S. Bandukwala, S. Oberdoerffer, B. Baust, Z. Li, V. Schmidt, P. G. Hogan, D. B. Sacks, and A. Rao, "Dephosphorylation of the nuclear 
factor of activated T cells (NFAT) transcription factor is regulated by an RNA-protein scaffold complex.," Proceedings of the National Academy of Sciences of the United States of America, vol. 108, pp. 11381-6, July 2011.

[164] F. Sabathe and P. Soucaille, "Characterization of the CipA Scaffolding Protein and In Vivo Production of a Minicellulosome in Clostridium acetobutylicum," Journal of Bacteriology, vol. 185, pp. 1092-1096, Feb. 2003.

[165] W. H. Schwarz, "The cellulosome and cellulose degradation by anaerobic bacteria," Applied Microbiology and Biotechnology, vol. 56, pp. 634-649, Sept. 2001.

[166] H.-P. Fierobe, E. A. Bayer, C. Tardif, M. Czjzek, A. Mechaly, A. Bélaïch, R. Lamed, Y. Shoham, and J.-P. Bélaïch, "Degradation of cellulose substrates by cellulosome chimeras. Substrate targeting versus proximity of enzyme components.," The Journal of biological chemistry, vol. 277, pp. 49621-30, Dec. 2002.

[167] C. You, S. Myung, and Y.-H. P. Zhang, "Facilitated Substrate Channeling in a SelfAssembled Trifunctional Enzyme Complex," Angewandte Chemie, vol. 124, pp. 89178920, Aug. 2012.

[168] K. Simons and D. Toomre, "Lipid rafts and signal transduction.," Nature reviews. Molecular cell biology, vol. 1, pp. 31-9, Oct. 2000.

[169] M. A. McCloskey and M. Poo, "Rates of membrane-associated reactions: reduction of dimensionality revisited," The Journal of Cell Biology, vol. 102, pp. 88-96, Jan. 1986.

[170] A. Rababa'h, J. W. Craft, C. S. Wijaya, F. Atrooz, Q. Fan, S. Singh, A. N. Guillory, P. Katsonis, O. Lichtarge, and B. K. McConnell, "Protein Kinase A and 
Phosphodiesterase-4D3 Binding to Coding Polymorphisms of Cardiac Muscle Anchoring Protein (mAKAP).," Journal of molecular biology, vol. null, June 2013.

[171] F. D. Smith, S. L. Reichow, J. L. Esseltine, D. Shi, L. K. Langeberg, J. D. Scott, and T. Gonen, "Intrinsic disorder within an AKAP-protein kinase A complex guides local substrate phosphorylation.," eLife, vol. 2, p. e01319, Jan. 2013.

[172] A. Perino, A. Ghigo, J. D. Scott, and E. Hirsch, "Anchoring proteins as regulators of signaling pathways.," Circulation research, vol. 111, pp. 482-92, Aug. 2012.

[173] S. Schuster, M. Marhl, and T. Höfer, "Modelling of simple and complex calcium oscillations," European Journal of Biochemistry, vol. 269, pp. 1333-1355, Mar. 2002.

[174] B. N. Kholodenko, "Negative feedback and ultrasensitivity can bring about oscillations in the mitogen-activated protein kinase cascades," European Journal of Biochemistry, vol. 267, pp. 1583-1588, Mar. 2000.

[175] E. Stefan, B. Wiesner, G. S. Baillie, R. Mollajew, V. Henn, D. Lorenz, J. Furkert, K. Santamaria, P. Nedvetsky, C. Hundsrucker, M. Beyermann, E. Krause, P. Pohl, I. Gall, A. N. MacIntyre, S. Bachmann, M. D. Houslay, W. Rosenthal, and E. Klussmann, "Compartmentalization of cAMP-dependent signaling by phosphodiesterase-4D is involved in the regulation of vasopressin-mediated water reabsorption in renal principal cells.," Journal of the American Society of Nephrology : JASN, vol. 18, pp. 199-212, Jan. 2007.

[176] A. Stangherlin and M. Zaccolo, "Local termination of 3'-5'-cyclic adenosine monophosphate signals: the role of A kinase anchoring protein-tethered phosphodiesterases.," Journal of cardiovascular pharmacology, vol. 58, pp. 345-53, Oct. 2011. 
[177] M. D. Houslay and D. R. Adams, "PDE4 cAMP phosphodiesterases: modular enzymes that orchestrate signalling cross-talk, desensitization and compartmentalization.," The Biochemical journal, vol. 370, pp. 1-18, Feb. 2003.

[178] S. Krishnamurthy, B. S. Moorthy, L. Xin Xiang, L. Xin Shan, K. Bharatham, N. K. Tulsian, I. Mihalek, and G. S. Anand, "Active site coupling in PDE:PKA complexes promotes resetting of mammalian cAMP signaling.," Biophysical journal, vol. 107, pp. 1426-40, Sept. 2014.

[179] B. S. Moorthy, Y. Gao, and G. S. Anand, "Phosphodiesterases catalyze hydrolysis of cAMP-bound to regulatory subunit of protein kinase A and mediate signal termination.," Molecular \& cellular proteomics : MCP, vol. 10, p. M110.002295, Feb. 2011.

[180] S. Seino, "Cell signalling in insulin secretion: the molecular targets of ATP, cAMP and sulfonylurea.," Diabetologia, vol. 55, pp. 2096-108, Aug. 2012.

[181] M. Iezzi, R. Regazzi, and C. B. Wollheim, "The Rab3-interacting molecule RIM is expressed in pancreatic $\beta$-cells and is implicated in insulin exocytosis," FEBS Letters, vol. 474, pp. 66-70, May 2000.

[182] K. Josefsen, Y. C. Lee, P. Thams, S. Efendic, and J. H. Nielsen, "AKAP 18 alpha and gamma have opposing effects on insulin release in INS-1E cells.," FEBS letters, vol. 584, pp. 81-5, Jan. 2010.

[183] D. Diviani, D. Maric, I. P. López, S. Cavin, C. D. del Vescovo, and I. Pérez López, "A-kinase anchoring proteins: Molecular regulators of the cardiac stress response.," Biochimica et biophysica acta, Aug. 2012. 
[184] T.-T. Aye, S. Soni, T. A. B. van Veen, M. A. G. van der Heyden, S. Cappadona, A. Varro, R. A. de Weger, N. de Jonge, M. A. Vos, A. J. R. Heck, and A. Scholten, "Reorganized PKA-AKAP associations in the failing human heart.," Journal of molecular and cellular cardiology, June 2011.

[185] E. Kanshin, L.-P. Bergeron-Sandoval, S. S. Isik, P. Thibault, and S. W. Michnick, “A Cell-Signaling Network Temporally Resolves Specific versus Promiscuous Phosphorylation.," Cell reports, vol. 10, pp. 1202-1214, Feb. 2015.

[186] J. Tröger, M. C. Moutty, P. Skroblin, and E. Klussmann, "A-kinase anchoring proteins as potential drug targets.," British journal of pharmacology, vol. 166, pp. 420-33, May 2012.

[187] Y. Wang, T. G. Ho, D. Bertinetti, M. Neddermann, E. Franz, G. C. H. Mo, L. P. Schendowich, A. Sukhu, R. C. Spelts, J. Zhang, F. W. Herberg, and E. J. Kennedy, "Isoform-selective disruption of AKAP-localized PKA using hydrocarbon stapled peptides.," ACS chemical biology, vol. 9, pp. 635-42, Mar. 2014.

[188] K. A. Ryall, D. O. Holland, K. A. Delaney, M. J. Kraeutler, A. J. Parker, and J. J. Saucerman, "Network reconstruction and systems analysis of cardiac myocyte hypertrophy signaling.," The Journal of biological chemistry, vol. 287, pp. 42259-68, Dec. 2012.

[189] R. P. Bhattacharyya, A. Reményi, B. J. Yeh, and W. a. Lim, "Domains, motifs, and scaffolds: the role of modular interactions in the evolution and wiring of cell signaling circuits.," Annual review of biochemistry, vol. 75, pp. 655-80, Jan. 2006.

[190] A. S. Khalil and J. J. Collins, "Synthetic biology: applications come of age.," Nature reviews. Genetics, vol. 11, pp. 367-79, May 2010. 
[191] J. E. Dueber, G. C. Wu, G. R. Malmirchegini, T. S. Moon, C. J. Petzold, A. V. Ullal, K. L. J. Prather, and J. D. Keasling, "Synthetic protein scaffolds provide modular control over metabolic flux.," Nature biotechnology, vol. 27, pp. 753-9, Aug. 2009.

[192] A. Lai, P. M. Sato, and S. G. Peisajovich, "Evolution of Synthetic Signaling Scaffolds by Recombination of Modular Protein Domains.," ACS synthetic biology, Jan. 2015.

[193] I. Sadowski, J. C. Stone, and T. Pawson, "A noncatalytic domain conserved among cytoplasmic protein-tyrosine kinases modifies the kinase function and transforming activity of Fujinami sarcoma virus P130gag-fps.," Molecular and cellular biology, vol. 6, pp. 4396-408, Dec. 1986.

[194] S. M. Lohmann, P. DeCamilli, I. Einig, and U. Walter, "High-affinity binding of the regulatory subunit (RII) of cAMP-dependent protein kinase to microtubule-associated and other cellular proteins.," Proceedings of the National Academy of Sciences of the United States of America, vol. 81, pp. 6723-7, Nov. 1984.

[195] D. Sarkar, J. Erlichman, and C. S. Rubin, "Identification of a calmodulin-binding protein that co-purifies with the regulatory subunit of brain protein kinase II.," $J$. Biol. Chem., vol. 259, pp. 9840-9846, Aug. 1984.

[196] A. C. Newton, "Analyzing protein kinase C activation," in Methods in Enzymology (R. Iyengar and J. D. Hildebrandt, eds.), vol. 345 of Methods in Enzymology, pp. 499506, Academic Press, 2002.

[197] A. S. Edwards and A. C. Newton, "Phosphorylation at Conserved Carboxyl-terminal Hydrophobic Motif Regulates the Catalytic and Regulatory Domains of Protein Kinase C," Journal of Biological Chemistry, vol. 272, pp. 18382-18390, July 1997. 
[198] W. G. Dunphy, R. J. Kochenburger, M. Castagna, and P. M. Blumberg, "Kinetics and Subcellular Localization of Specific [3H]Phorbol 12,13-Dibutyrate Binding by Mouse Brain," Cancer Res., vol. 41, no. 7, pp. 2640-2647, 1981.

[199] N. Ward and C. O'Brian, "Kinetic analysis of protein kinase C inhibition by staurosporine: evidence that inhibition entails inhibitor binding at a conserved region of the catalytic domain but not competition with substrates," Mol. Pharmacol., vol. 41, pp. 387-392, Feb. 1992.

[200] G. Martiny-Baron, M. Kazanietz, H. Mischak, P. Blumberg, G. Kochs, H. Hug, D. Marme, and C. Schachtele, "Selective inhibition of protein kinase C isozymes by the indolocarbazole Go 6976," J. Biol. Chem., vol. 268, no. 13, pp. 9194-9197, 1993.

[201] K. M. Lin, B. Lin, I. Y. Lian, R. Mestril, I. E. Scheffler, and W. H. Dillmann, "Combined and Individual Mitochondrial HSP60 and HSP10 Expression in Cardiac Myocytes Protects Mitochondrial Function and Prevents Apoptotic Cell Deaths Induced by Simulated Ischemia-Reoxygenation," Circulation, vol. 103, pp. 1787-1792, Apr. 2001.

[202] Z. Diwu, J. Zimmermann, T. Meyer, and J. Lown, "Design, synthesis and investigation of mechanisms of action of novel protein kinase $\mathrm{C}$ inhibitors: perylenequinonoid pigments," Biochemical Pharmacology, vol. 47, pp. 373-385, Jan. 1994.

[203] N. Grodsky, Y. Li, D. Bouzida, R. Love, J. Jensen, B. Nodes, J. Nonomiya, and S. Grant, "Structure of the catalytic domain of human protein kinase C beta II complexed with a bisindolylmaleimide inhibitor.," Biochemistry, vol. 45, pp. 13970-81, Nov. 2006. 
[204] S. Schauble, C. C. King, M. Darshi, A. Koller, K. Shah, and S. S. Taylor, "Identification of ChChd3 as a novel substrate of the cAMP-dependent protein kinase (PKA) using an analog-sensitive catalytic subunit.," The Journal of biological chemistry, vol. 282, pp. 14952-9, May 2007.

[205] J.-H. Kang, D. Asai, S. Yamada, R. Toita, J. Oishi, T. Mori, T. Niidome, and Y. Katayama, "A short peptide is a protein kinase C (PKC) alpha-specific substrate.," Proteomics, vol. 8, pp. 2006-11, May 2008.

[206] S. Gandy, A. J. Czernik, and P. Greengard, "Phosphorylation of Alzheimer disease amyloid precursor peptide by protein kinase $\mathrm{C}$ and $\mathrm{Ca} 2+/$ calmodulin-dependent protein kinase II.," Proceedings of the National Academy of Sciences of the United States of America, vol. 85, pp. 6218-21, Aug. 1988.

[207] J. A. Adams, M. L. McGlone, R. Gibson, and S. S. Taylor, "Phosphorylation modulates catalytic function and regulation in the cAMP-dependent protein kinase.," Biochemistry, vol. 34, pp. 2447-54, Feb. 1995.

[208] B. D. Grant, W. Hemmer, I. Tsigelny, J. A. Adams, and S. S. Taylor, "Kinetic analyses of mutations in the glycine-rich loop of cAMP-dependent protein kinase.," Biochemistry, vol. 37, pp. 7708-15, May 1998.

[209] C. S. Gibbs and M. J. Zoller, "Identification of electrostatic interactions that determine the phosphorylation site specificity of the cAMP-dependent protein kinase.," Biochemistry, vol. 30, pp. 5329-34, June 1991.

[210] W. Zhang, G. Z. Morris, and S. J. Beebe, "Characterization of the cAMP-dependent protein kinase catalytic subunit Cgamma expressed and purified from sf9 cells.," Protein expression and purification, vol. 35, pp. 156-69, May 2004. 
[211] B. Zhou, Z.-X. Wang, Y. Zhao, D. L. Brautigan, and Z.-Y. Zhang, "The specificity of extracellular signal-regulated kinase 2 dephosphorylation by protein phosphatases.," The Journal of biological chemistry, vol. 277, pp. 31818-25, Aug. 2002.

[212] H. Yamaguchi, G. Minopoli, O. N. Demidov, D. K. Chatterjee, C. W. Anderson, S. R. Durell, and E. Appella, "Substrate specificity of the human protein phosphatase 2Cdelta, Wip1.," Biochemistry, vol. 44, pp. 5285-94, Apr. 2005.

[213] C. W. Dessauer and A. G. Gilman, "The Catalytic Mechanism of Mammalian Adenylyl Cyclase: EQUILIBRIUM BINDING AND KINETIC ANALYSIS OF P-SITE INHIBITION," Journal of Biological Chemistry, vol. 272, pp. 27787-27795, Oct. 1997. 